\title{
LEGIBINTY
}

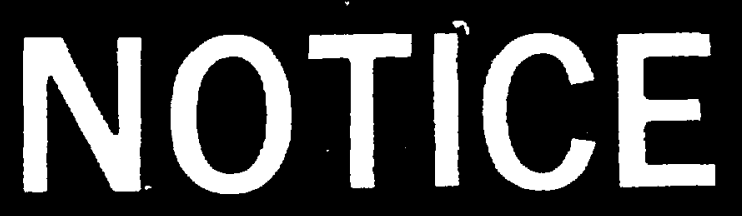

A major purpose of the Technical Information Center is to provide the broadest dissemination possible of information contained in DOE's Research and Development Reports to business, industry, the academic community, and federal, state and local governments.

Although a small portion of this report is not reproducible, it is being made available to expedite the availability of information on the research discussed herein.

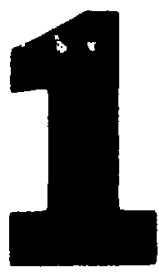


$\frac{136}{6 / 1} / 19985(3)$

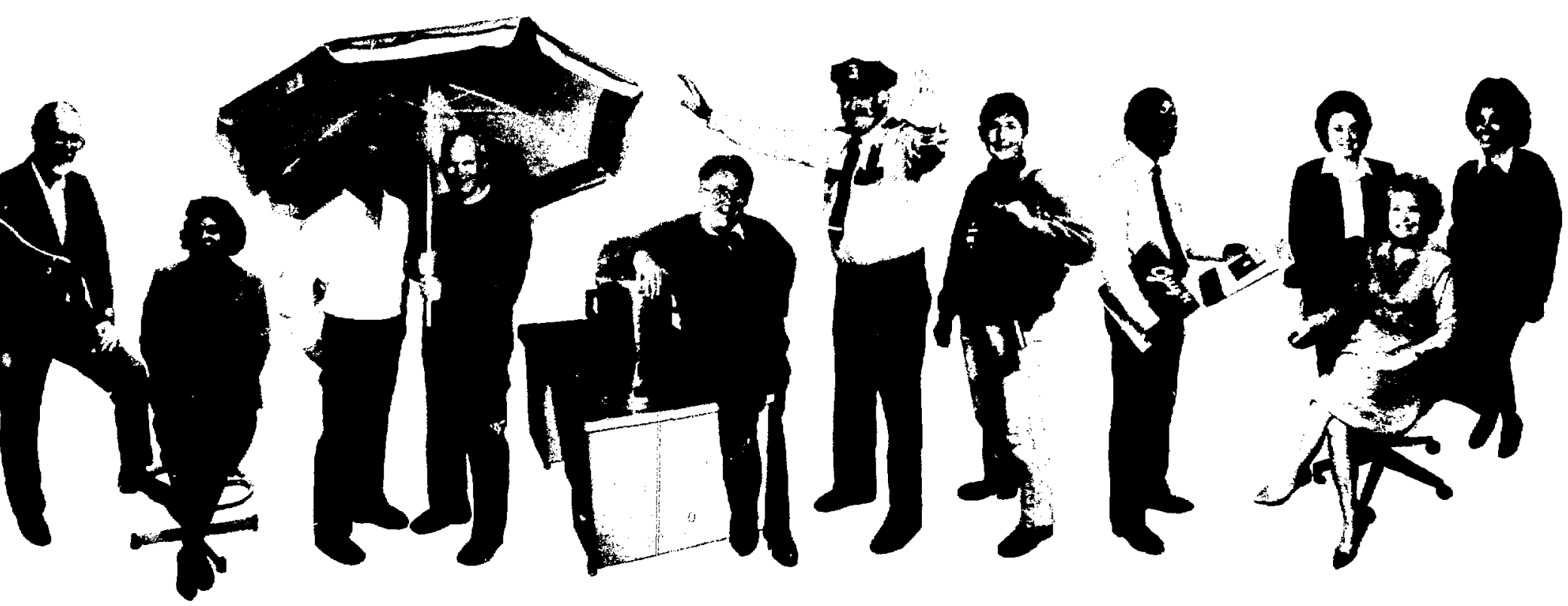

$\begin{array}{lllllllllllllllllllll}\text { B } & \mathbf{R} & \mathrm{O} & \mathrm{O} & \mathbf{K} & \mathrm{H} & \mathbf{A} & \mathrm{V} & \mathbf{E} & \mathrm{N} & & \mathrm{H} & \mathrm{I} & \mathrm{G} & \mathrm{H} & \mathrm{L} & \mathrm{I} & \mathrm{G} & \mathrm{H} & \mathrm{T} & \mathrm{S}\end{array}$

Brookhaven National Laboratory

MASTER

DISTRIBUTION OF THIB DOCUNIENT S LINUIMITED 
BNL --52165

DE89 012793

\title{
DISCLAIMER
}

This report was prepared as an account of work sponsored by an agency of the United States Government. Neither the United States Government nor any agency thereof, nor any of their employees, makes any warranty, express or implied, or assumes any legal liability or responsibility for the accuracy, completeness, or usefulness of any information, apparatus, product, or process disclowed, or represents that its use would not infringe privately owned rights. Reference herein to any specific commercial product, process, or service by trade name, trademaik, manufacturer, or otherwise does not necessarily constitute or imply its endursement, recommendation, or favoring by the United States Government or any agency thereof. The views and opinions of authors expressed herein do not necessarily state or reflect those of the United States Government or any agency thereof.

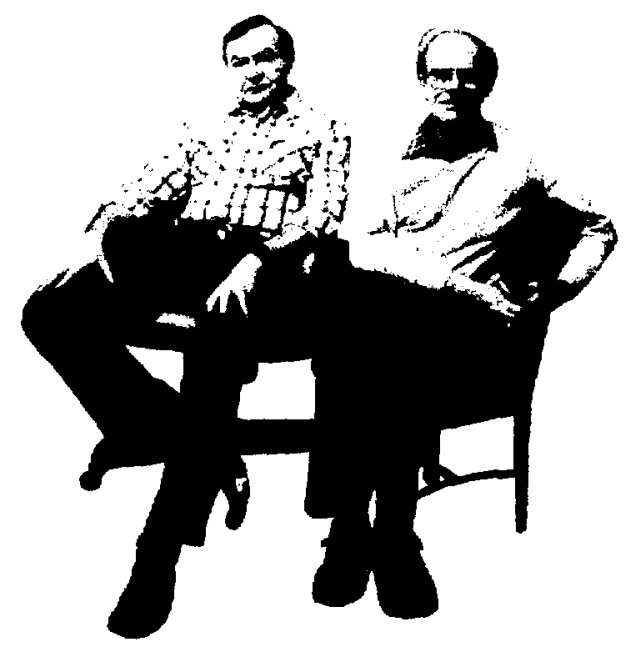

\section{Brookhaven Highlights}

\author{
Brookhaven National Laboratory \\ Upton, Long Island, New York 11973
}

This report highlights the Laboratory's activities for fiscal year 1988 , the period dating from October 1, 1987, through September 30, 1988. 


\section{Contents}

2 A Word About BNL.

4 Brookhaven Today

6 Looking Forward

12 The Big Machines

15 Alternating Gradient Synchrotron Department 16 Farewell, Cockcroft-Walton - Hail, New RFQ!

18 A Search for Eventful Physics

20 Forbidden Violations

\section{Accelerator Development Department}

24 High Marks for the Booster

26 An Experiment With a Ring to It

29 Physics Department

30 The Puzzle of the Positrons

31 Filling the Holes in High $-T_{c}$ Theory

33 Testing the Standard Model

35 Department of Nuclear Energy

36 Answer's in Sloort Time

38 A Technical Knockout

40 Accelerator Technology for Defense

43 Department of Applied Science

44 Polymers for the Future

46 Putting the Brakes on Accjdents

48 The Greenhouse Effect: About FACE

51 National Synchrotron Light Source Department

52 Breakthrough in Chip Technology

53 Orbits, Spins and Resonance:

An Elegant Experiment

55 Innovative Optics at the Light Source

57 Chemistry Department

58 The Energetic Molecules

60 Exploring Molecular Energy Conversion

62 The Great Solar Neutrino Trap

65 Medical Department

66 Using PET to Study Cocaine Abuse and Brain Function

68 Hope for Incurable Cancers

69 Radon: Assessing Low-Level Exposure
71 Biology Department

72 The Enigmatic Enzymes

74 Unravelirg the 30 -nm Filament

75 Hatchet Men of the Pneumonia Bacteria

77 Support Activities

78 Applied Mathematics Department

79 Multiplication Leads to Division

80 Reactor Division

80 Spin in Supereonductors

82 Safety and Environmental Protection Division

82 Keeping Watch Over the Environment

84 Instrumentation Division

84 Thinking Tiny

\section{Laboratory Profile}

88 Meetings

90 Honors

92 Administration

95 Financial Report

96 Organization 


\section{A Word About BNL}

$A_{\text {quick pass arer Brootharen }}$ National Laborarory would be enough to show that this is no ordinary Long lsland community. Fven from the air, the huge elipse ready. to house the proposed Relativistic lleary Ion Collidere, the smaller circle harboring the Alternating Gradient Sinchrotron, the rounded angles of the National Synchrotron Light source and the gleaming dome of the High Flux Beam Reactor mark Brookhaven as a special place.

But it is not the buildings that make Brookhaven special. Rather, it is the spect acular science that is done inside these buildings. From its "big machines" to its myriad research laboratories, B.NL brings the resources of academia and the federal government together, to carry out research endeavors not normally within the capability of a single university.

Brookhaven is a multidisciplinary laboratory that carries out basic and applied research in the physical, biomedical and environmental sciences and in selected energy technologies. All this is done under the managenome of Associated l'niversit iss. Inc. (All), which was formed in 1946 by a group of nine universities, for the purpose of establishing and operating a major scientific laboratory in the Northrast. In January 1947, BNL Was a fact.

Today, All cont inues to manage BNL under a cont ract with the I'S. Depart ment of Energy. The nine sponsoring universities are Columbia Iniversity, Cornell Iniversity. Harvard Iniversity, The Johns Hopkins I'niversity, Massachusetts Institute of Technology. Iniversity of Pennsylvania, Princet on University, Lniversity of Rochester and Yale Liniversity.

Brookhaven is located on Long Island, at Upton, New York, the site of a former Army camp where American soldiers trained during World Wars I and II. Now, some 3,200 civilian employees help the science go forward at a site that resembles a sprawling university campus, containing about 300 buildings and other structures on 5,265 acres of wooded property.

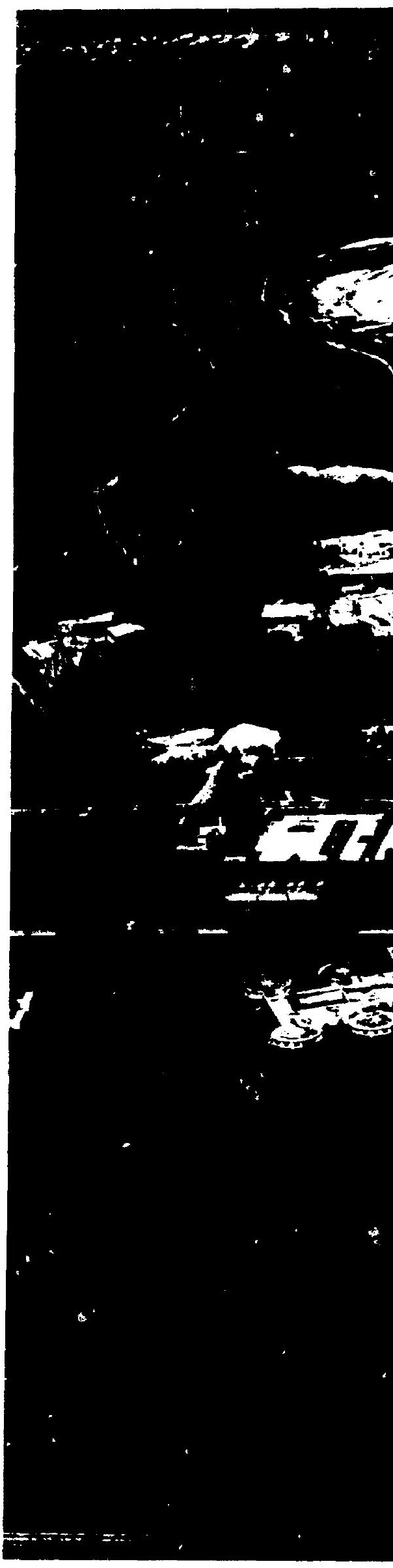




\section{Brookhaven Today}

W nize it as one of those years of studded with a constant stream of milestomes marking Brokhaven's involvement in a great variety of exciting and expanciing scientific activities. We were able to achieve such diversity of accomplishment at our multidisciplinary laboratory thanks to our most important asset - our people.

You will see our people throughout this issue of Brookhaven $\mathrm{High}$ lights. When you read about the work done over this past year, you'll realize just what a multitalented staff Brookhaven truly has - proud of our achievements and looking forward to doing even better. And we're involved.

With sound plans for the future, we at Brookhaven are all involved at the cutting edge of basic science and technology. In 1988, this langed from experiments at the Alternating Gradient Synchrotron (AGS) looking at the rare decays of particles called kaons, to studies investigating the whys of high temperature superconductivity, at the High Flux Beam Reactor (HFBR) and the National Synchrotron Light Source (NSLS).

To make possible such forefront research, we're involved with providing our user community with the best facilities for the job. In 1988, we devoted considerable energy to making our already widely respected facilities even better.

Though the extensive upgrade of the NSLS $x$-lay ring continues, by the end of the year, light had returned to the ring and the new insertion devices were nearing completion. Likewise, improvements to the AGS included the July dismanthing of the Cockcroft-Walton that had so faithfully preaccelerated protons for 28 years, to make room for a state-of-the-art radiolreguency quadrupole ( $R F(2)$ injector that promises many nore year's of fruit ful research at the Ags. Of course. eonstruction continued on the Accumulator-Booster, which will greatly enhance the Acis program.

The Booster also will make it possible for the AGs to serve as an injector for the Relativist ic Heavy Ion Collider (RHIC) that we have proposed to serve a combined community of high energy and nuclear physicists. Other good news for RHIC came in February, when the Tandem Van de Graaff, which injects heavy ions into the AGS, produced a gold beam even more intense than that needed to meet RHIC's specifications, and in May, when the first superconducting quadrupole magnet passed its tests with flying colors.

The future of the user program at the HFBR looked even brighter when, in June, the U.S. Department of Energy (DOE) recommended that needed improvements to the reactor's instruments be implemented. Work began this year on an Accelerator Test Facility to validate advanced concepts for accelerators, and users of BNL's Positron Emission Tomography (PET) facility welcomed a new whole-body PET machine in June.

We're also involved in responding to national needs. In 1988 , we received initial funding from a fiveyear, $\$ 21$-million contract with the federal government to develop a superconducting $x$-ray lithography source for making compute $\ddot{x}$ chips. Ultimately, the final technology will be transferred to industry. Thus, we will fulfill the fundamental goal of our technology transfer efforts -.- to get the products of our research out into the private sertor - while helping to assure that the l's. will maintain its technological rompet itiveness in the international market. 
Other Brookhaven projects also have national scope. We are a prime cont ributor to plans for the proposed superconducting Super Collider, including construction of the superconducting magnets. Our researchers went to lllinois in July to participate in the precedent set ting field experiment to study the phenomenon of acid rain through the chemist ry and physics of thunderstorms in a polluted environment. And in May, we celebrated the dedication of the Laboratory's Neutral Beam Test Facility, which, along with our Radiation Effects Facility, is Brookhaven's contribution to the federal government's Strategic Defense Initiative.

At the international level, over twenty years ago, Brookhaven saw the necessity for safeguards in atomic energy. The result was BNL's Technical Service Organization, which celebrated its 20 th anniversary in September and looks forward to many more years of helping to assure the planet's nuclear safety.

We're also involved with the local community. For example, through an agreement with Suffolk County, we have opened the Laboratory to the county, so they can participate in the environmental monitoring of our research activities.

Our main business, however, is science, so that's what most of our community contacts are geared to. Through site tours and our continually growing educational programs, students from elementary school through graduate school, senior citizens, industrial groups and others all have an opportunity to learn about the world of science.

We're involved with Associated Universities, Inc. The partnership that began when ALI formed Brookhaven has continued uninterrupted since 1947. And its further continuance was assured in December, when DOE renewed its contract with Al I to manage the Laboratory through 1992.

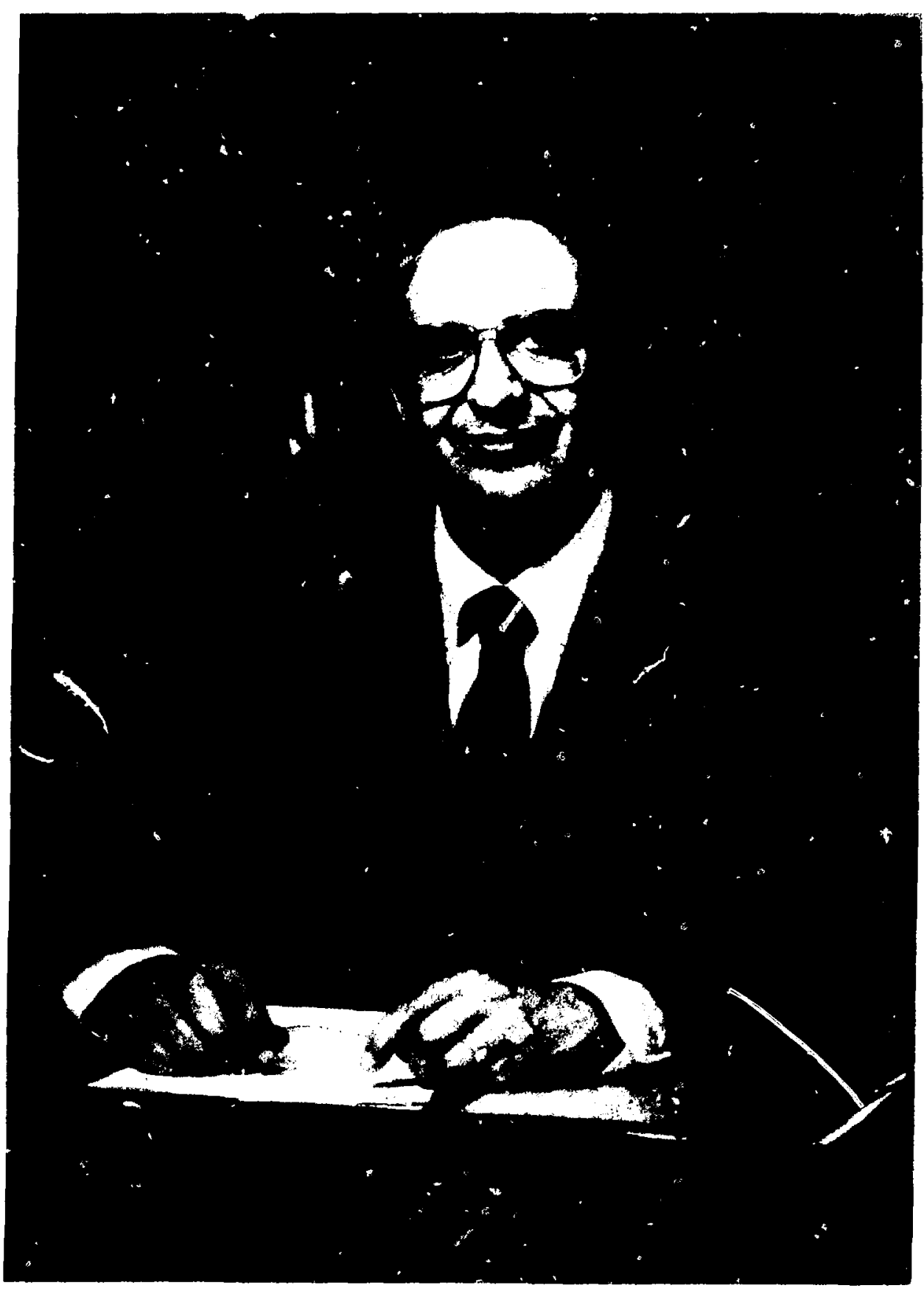

Yes, we're involved with everything - especially the future. And with our world-class facilities - and especially our people - I know we will meet all the scientific challenges that lie ahead.

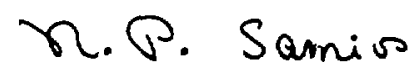

Nicholas P. Samios Director 


\section{Looking Forward}

The quest for knowledge never ends. In 1987, Brookhaven celebrated its 40th anniversary, an occasion to reflect on the landmark discoveries made over the past forty years. The year 1988 marked the beginning of the next forty years, with the Laboratory looking forward to continuing achievement in many different areas.

\section{Relativistic Heavy Ion Collider} (RHIC) - "Brookhaven has long been distinguished by its innovative and precedent-setting ideas on new types of particle accelerators. Continuing in this tradition, $\mathrm{RHIC}$, the Relativistic Heavy Ion Collider proposed by the Laboratory, will reach far beyond the energies achieved by any existing nuclear physics facility. By creating conditions which have probably not existed in the universe since the Big Bang, we will be able to study the fundamental properties of matter in a state in which the primordial quarks and gluons are no longer confined as constituents of rdinary particles. Once achieved, this so-called quark matter offers a whole new area of scientific study for nuclear physics as well as elementary particle physics, and it will

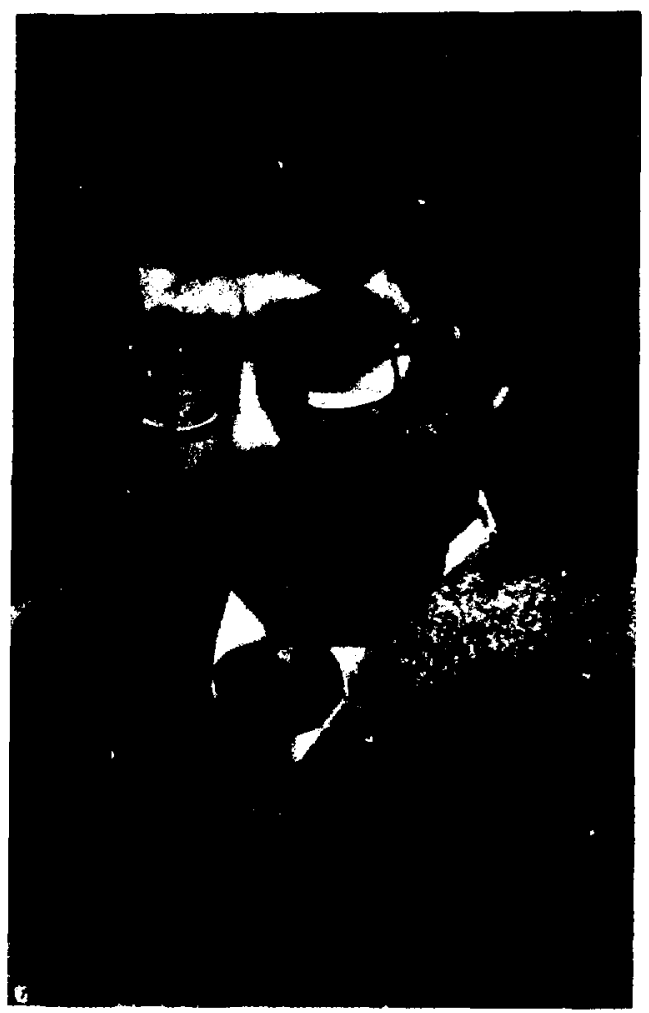
also give essential new information regarding the early stages and subsequent evolution of our universe.

"RHIC will stand as yet another scientific achievement made possible by the AGS, the Alternating Gradient Synchrotron, which will serve as the injector for RHIC. Already, with the acceleration of heavy ion beams from the Tandem, the AGS has opened up a heretofore unexplored field of research whose potential for new discoveries has made it a central part of the U.S. nuclear physics program. As one of the world's premier high energy proton accelerators, the AGS has had an extraordinarily long and fruitful history of scientific discovery. With completion of the Booster and future improvements such as the addition of a stretcher ring, the AGS will continue to be one of this country's foremost high energy physics lacilities for some time to come." 


\section{Magnetically Levitated Trains -}

"In Ietsti. ciordon l banby and I published the first paper on the use of superconducting magnets to levitate high-speed trains, and in 1968, we were granted the original patent on supereonducting masnetically levitated trains. Today, the Japanese have demonstrat ed the feasibility of 300-mile-per-hom levitated trains. This technology could revolutionize the transportation system in the Cnited states, which is already heavily scressed and getting worse."

James Powell, Nuclear Engineer

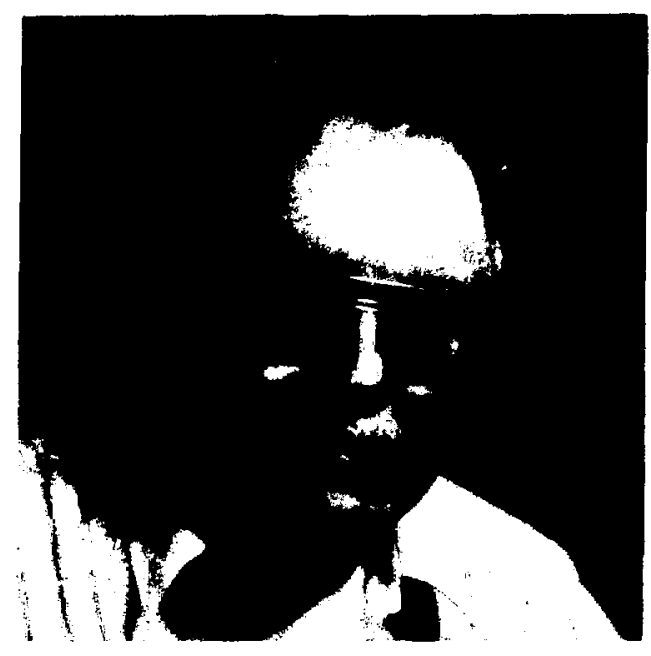

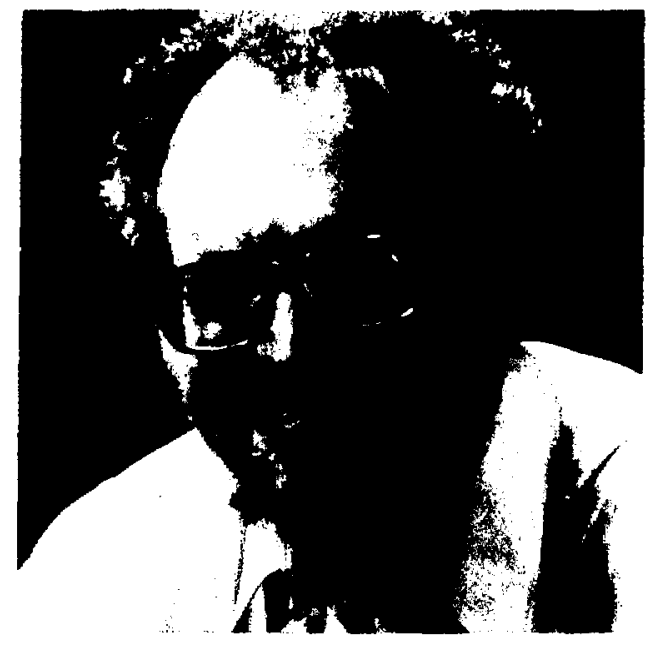

"We think that a mag-ley system can act ually be implemented within five year's. The challenge is to develop designs that use low-cost, massproduced track sections that can be inst alled along existing rights of way with minimum fiekd construction. We believe that l's. indust ry can meet the challenge, given adequate resourcess and encouragement."

Gordon Danby, Physicist

Funding From Non-DOE Sources - "In view of the change in administrations, budgetary constraints and greater concern for protecting and cleaning up the environment, I envision a resurgent interest in nuclear power in the next few years. There will be even more emphasis on safety, both in plant design and operation. Therefore, I ant icipate continued strong nuclear safety programs at Brookhaven in the area of safety research and technical support for the l's. Nuclear Regulatory Commission. In addition, with the improvement of relations between the Soviet l'nion and the l's., there will be increased emphasis on arms reductions, coupled with a greater need for sophisticated verification techniques and methodology. In light of these international developments, I expect the Laboratory will see increasing support from various agencies for studies on arms cont rol."

Walter Kato, ('hairman, Iepartment of Nuclear Energy'

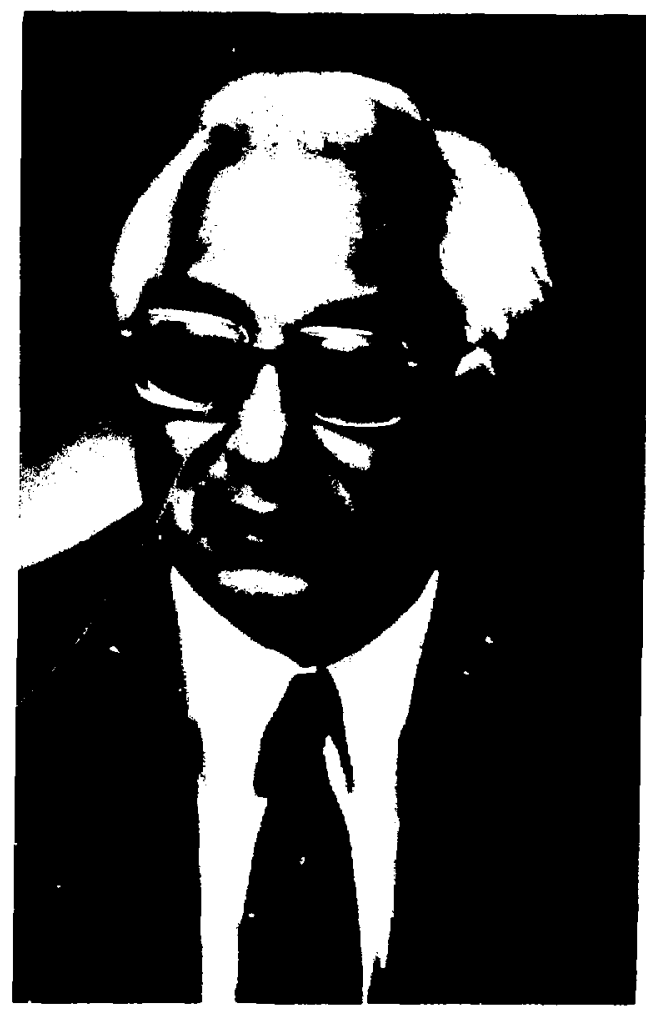




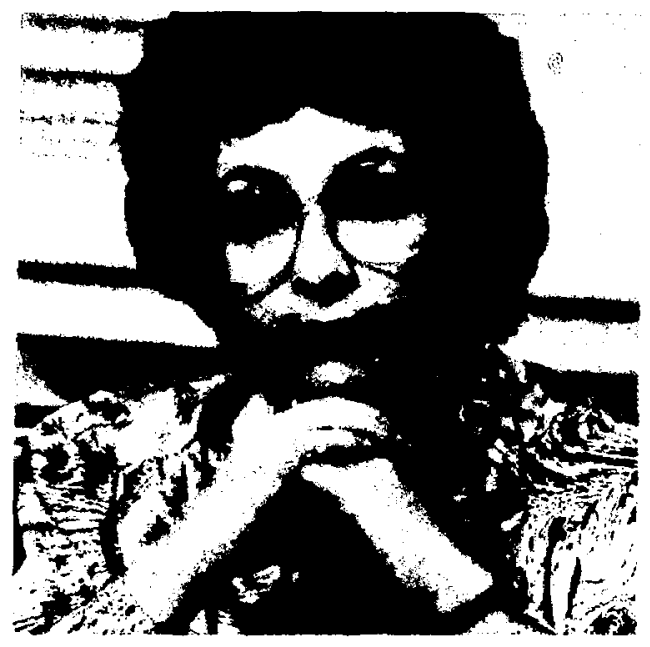

Training and Development - "For Brooklaven and ot her R\&I organizations, training is becoming essential to maint aining a highly skilled work force. With changing demographics, we expect that competition for technically skilled and scientific employees will increase dramatically. We will be taking an increasingly active role in developing the expertise that is essential to our mission. At the same time, we will likely be encouraging employees to diversify and extend their careers, as the number of technically trained men and women graduating from colleges and universities declines. Retraining will also be required to overcome skill obsolescence that would otherwise result from rapidly changing technology and an aging population. Just as important will be the development of our managers, whose leadership will enable the Laboratory and its employees to meet the challenges of the future."

Mary White, Training and Developmeni Administrator Personnel Division

\section{Radiation Therapy - "Plans are under way for a joint BNL SLNY} Stony Brook radiation therapy facility, which is expected to be operational by fiscal year 1991. This facility will provide state-of-the-art radiation therapy services to Stony Brook University Hospital patients who come from eastern Suffolk County. In addition, the facility will be the focal point for clinical applications of the promising radiation therapies currently under investigation in the Medical Department."

Arjun Chanana, Chairman Medical Department

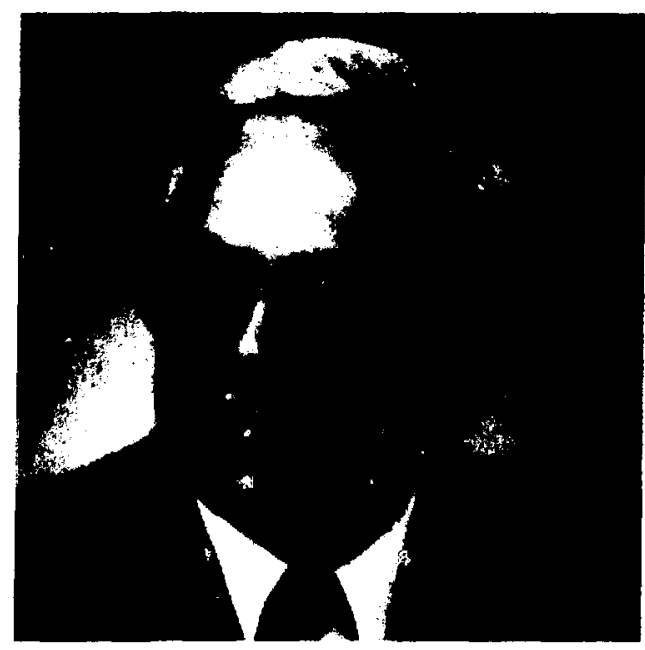

Compact Synchrotron for X-Ray Lithography - "Support of the research and technology goals of U.S. industry is one of the key missions of the Laboratory today. At the National Synchrotron Light Source, we've just begun a five-year project to construct the first compact synchrotron for $\mathrm{x}$ ray lithography in the United States. $X$-ray lithography is a new technology that promises to revolutionize the manufacture of computer chips. The challenge ahead is twofold: to develop the technology and to transfer the know-how to industry. This project is critical to bolstering the domestic semiconductor industry and to establishing domestic vendors of compact synchrotrons dedicated to $x$-ray lithrography. We're excited about being a key player in helping to ensure U.S. competitiveness in the world computer chip market."

Michael Knotek, Chairman National Synchrotron Light source

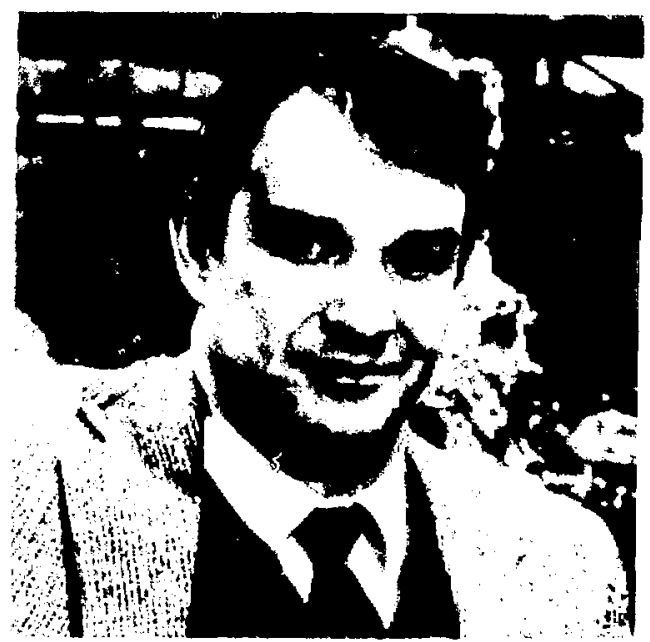


Indust rial Safety and Health "Health and satfery regulations in the fut ure will be drisen more by public perception of risk than hy scientitically defined risk assessments. New safery standarels will also repuire more document at ion and quality assurance to prove eompliance.

What does this mean for the scientific community": In effect, we will be challenged to interpret our highty technical observations in lay terms and to be more sensitive in our responsibility to inform the public."

Otto White Jr

Associate Division Head Safely and Environmental Protection IDicision

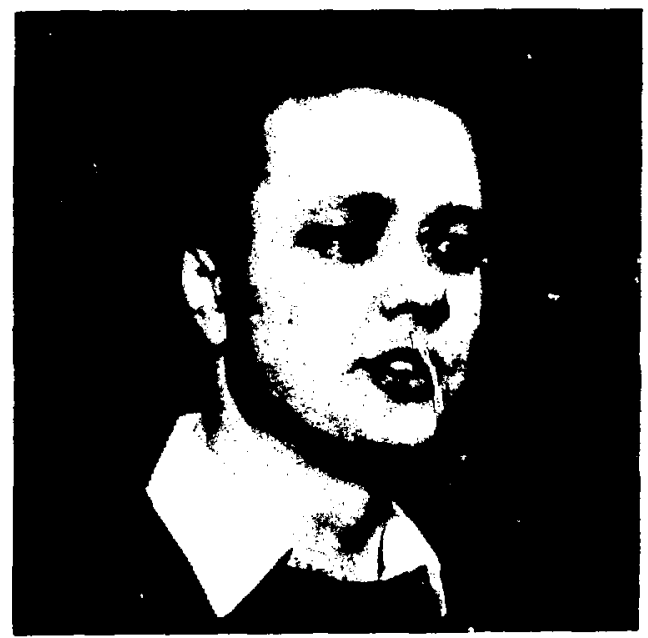

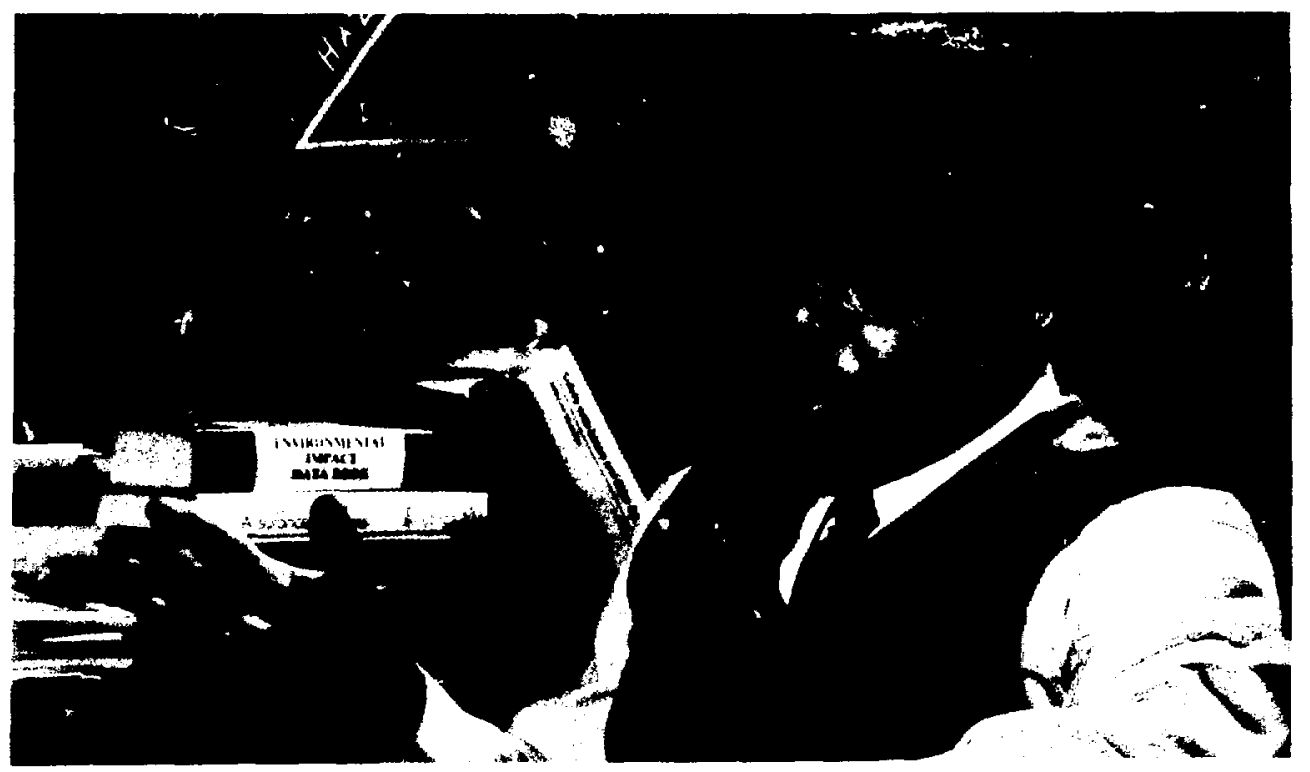

Users - "Lsers have always been important to the function of many research efforts at BNL, and as such are given strong Lab support. On many AGs experiments, for example, the majority of participants are from outside institutions. Strong financial and technical backing provided by BNL has made these experiments some of the most successful in their fields, and with a solid Lab commitment to upgrade and improve existing AGS facilities, the accelerator will continue to produce world-class physics. And speaking from personal experience, the Lab's assistance to outside users and their families in terms of general 'quality of living' is very good, ranging from an on-site apartment community complete with nursery school, to BNL-sponsored activities for families living at the Lab."

Mats Selen, Physicist, Princeton University
Biotechnology - "Biotechnology at B.NL is an interdepartmental interdisciplinary effort. A major thrust is to elucidate the relations among the structures and functions of macromolecules, not only to understand how cells and organisms work, but also to engineer useful organisms and make better-working molecules to improve human health and well-being. Also of interest is the identification and design of microorganisms that will be useful in environmental purification and cleanup of toxic metals and organic cont aminants from wastes. We project strong growth in biotechnology programs at Brookhaven, particularly bec'ause of our unique lacilities for struct ural and cellular biology, including the High Flux Beam Reactor, the National Synchrot ron Light Source, which also has an x-ray microprobe, and the Scannirg Transmission Elect ron Microscope."

Richard Set low, Assoriate Director for Life Sciences

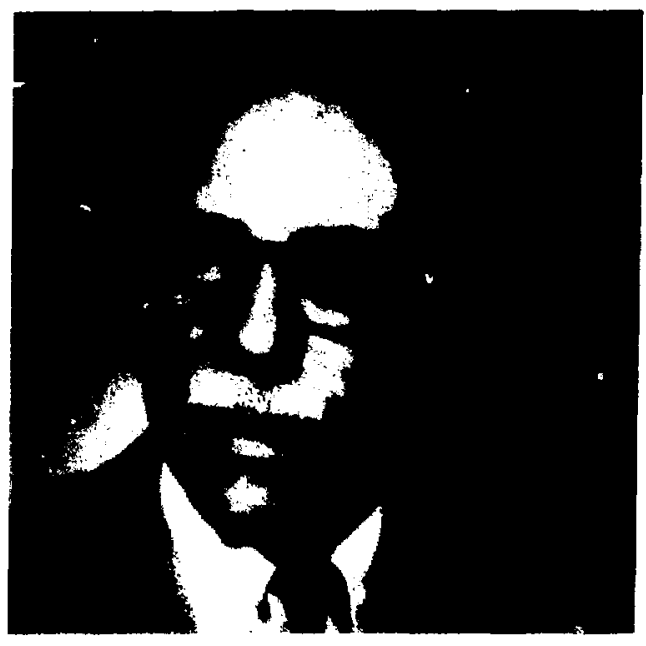




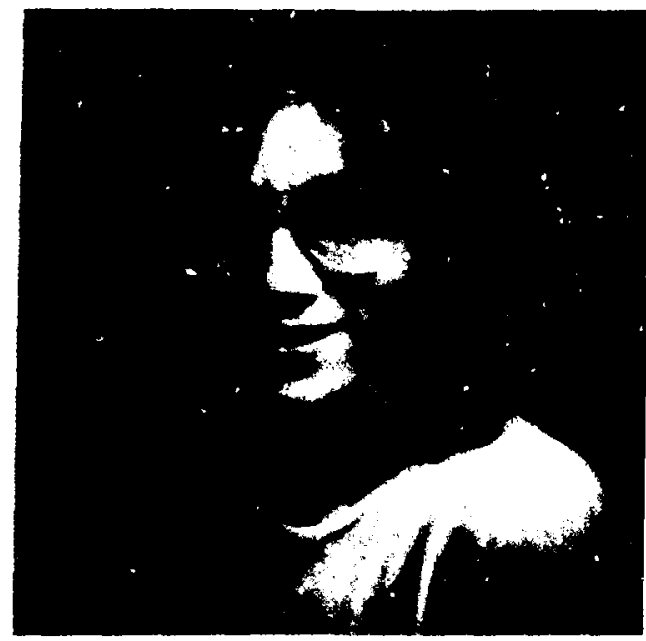

PET (Positron Emission Tomography) - "It is safe to say that basic research and the application of high technology has shaped the PEI field as we know it today. PET is now rapidly moving into clinical practice, where it already provides unique diagnostic information used in the care of patients with heart disease, cancer and epilepsy. With the current development of new radiotracers to probe the biochemical abnormalities underlying mental illness and the basic neurochemical changes that occur with substance abuse, we can anticipate a new generation of more effective, safer drugs and other treatments of disease and problems of addiction. To effectively use Pl:'s unique high-technology resources and trained personnel, regional centers will be used increasingly to supply short-lived radiot racers to hospitals to complement the stand-alone cyclotron-PET facilities, the number of which is expected to double in the next five years. These regional centers will also serve to train scientists required for this new and unique specialty in the health care system."

Joanna Fowler, Chemist, Cyclotron-PET Program

BNL Site - "The keystone to general site development in the next decade is a new Research Support Facility. This will be a multistory building on the quadrangle between Brookhaven and Cornell Avenues. It will house many of the Laboratory's administrative and support functions. When this building goes up, we expect to be able to get rid of several of the outdated wooden Army buildings. If we are sucressful in funding new space for the Departments of Applied Science and Nuclear Energy, even more of these old buildings can be eliminated. We also look forward to additional phases to the Central Chilled Water Facility, so that most of our major buildings can be airconditioned with efficient and reliable distributed cooling water."

Alfred Mahlmann, Manager

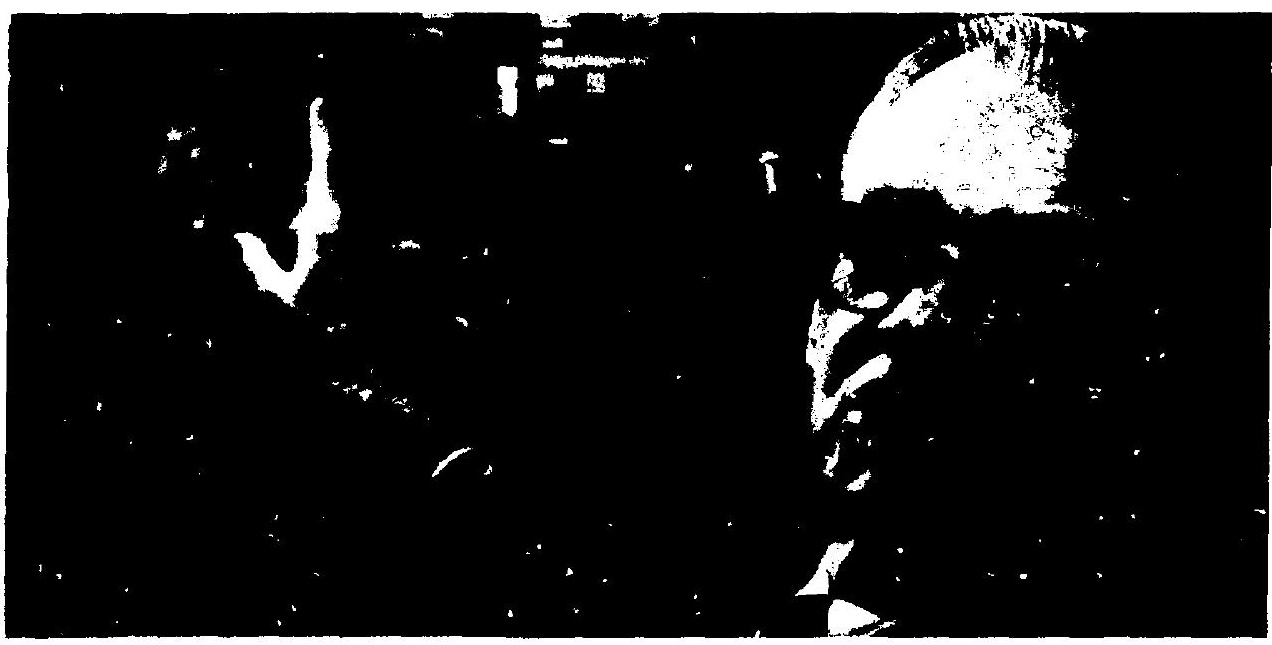
Plant Engineering Division

Materials Sciences - "Research in materials sciences is an important component of the overall Laboratory effort. The unique facilities of BNL the National Synchrotron Light Source, the High Flux Beam Reactor and the Scanning Transmission Electron Microscope - provide a basis for major research efforts in materials. Already, the reactor, for example, has produced seminal discoveries in the mechanisms of the new high temperature superconductors. In the next decade, BNL researchers as well as our many outside users will make important advances in understanding the mechanisms and phenomena of materials properties. These efforts will be at the forefront of basic science as well as in the vanguard of practical application."

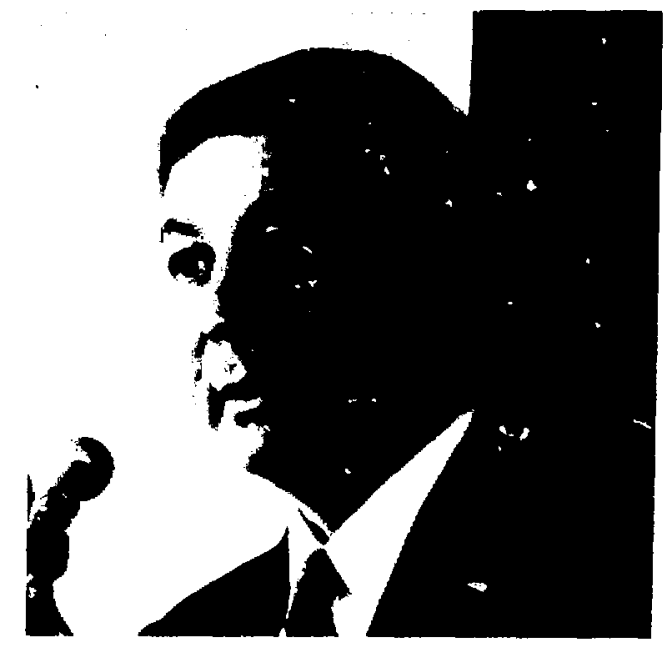



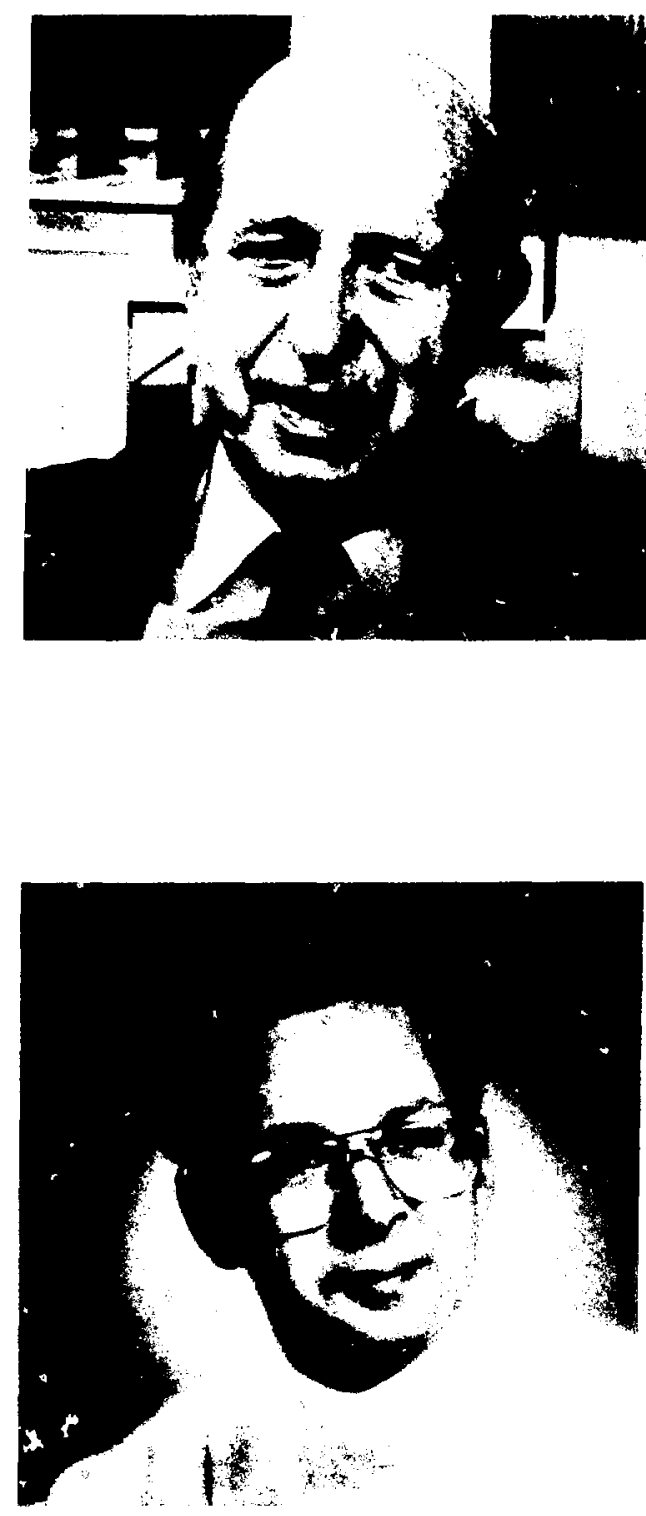

Technology Transfer - "If the l's. is to improve it ind ist rial st rength and its international competitiveness, it is important that Brookhaven play a major role in transferring technology developed at taxpayer expense to the industrial sector. The Laboratory has already made some major st rides by transferring our technology in $x$-ray lithography to the semiconductor industry, our technology in developing cost-effective energy applications to the electric utility industry, as well as instrumentation and radioactive materials for the medical and pharmaceutical industry.

"In the next decade, we anticipate our present initiatives will be expanded in the development of high-temperature superconducting materials, conducting polymers, catalysts, and in the study of materials such as biological macromolecules. We expect increased participation by our scientists and engineers with the industrial research community through consultation, collaborative research programs, and in proprietary research. We must see to it that the valuable resources at Brookhaven are available to the industrial sector."

Seymour Baron, Associate Director for Applied Programs
Accelerator Test Facility - "The Center for Accelerator Physics was created to consider all kinds of far-out accelerator ideas. We are now constructing a small linac, known as the Accelerator Test Facility. The plan is to do many experiments ... for example, to use a powerful laser to accelerate to high energies in quite short distances, and to back-scatter laser light from the beam to produce a new source of photons. Because such: ideas will benefit different areas of science, our members come from valious departments such as Instrumentation, Light Source, Physics and Accelerator Development. It is only in a multidisciplinary lab like BNL that this is possible."
Robert Palmer, Joint Director Center for Accelerator Physics

Budget - "Although FY 1988 began without a Congressional appropriation, we ended the year in a reasonably strong financial position. Looking ahead, FY 1989 promises to be another good year for the Laboratory as a whole. For the first time, BNL's budget will exceed $\$ 300$ million. That reflects a strong commitment on the part of the Department of Energy to properly operate our major user facilities and to maintain and improve the Laboratory's infrastructure.

"As for the years to come, the future looks promising. Our plans assume that RHIC will be built and that several other enhancements to our major user facilities will be initiated. However, we also forecast increased costs of operating the Laboratory. For example, we are keenly aware of environmental, safety and health issues and have developed a long-range plan that will allow us to get well and stay well. In order to achieve this, we must commit considerable funding to these activities."

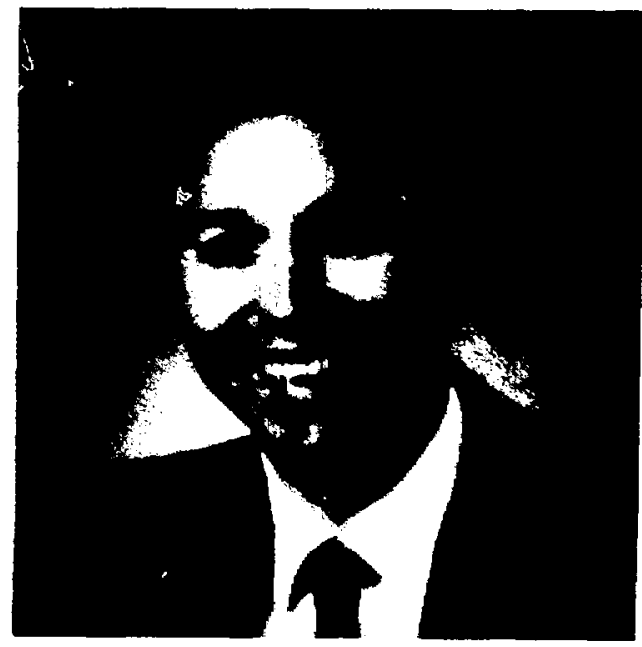




\section{The Big Machines}

An observer's first impression of Brookhaven's "Big Machines" is usually related to their overwhelming size. Later comes a fuller realization of the many small parts that make up the huge whole - the hundreds of different experiments that may be running inside the facility - the hundreds of thousands of components of the apparatus - and the extreme accuracy needed in every component.

At work on the experiments are teams of Brookhaven scient ists and. over the course of a year, about 2.800 researchers and students from many universities, indust ries and other laboratories. The extraordinary range of the studies performed at these large-scale research facilities promotes a flow of ideas and cooperation between the many users and creates an entironment conducive to exciting science.

The Office of Research and Technology Applications can assist industrial researchers who wish to use the facilities. Outside users may retain title to inventions or dat a generated during wolk at Brook haven by entering into a proprietary user's agreement with the Laboratory.

\section{National Synchrotron Light Source}
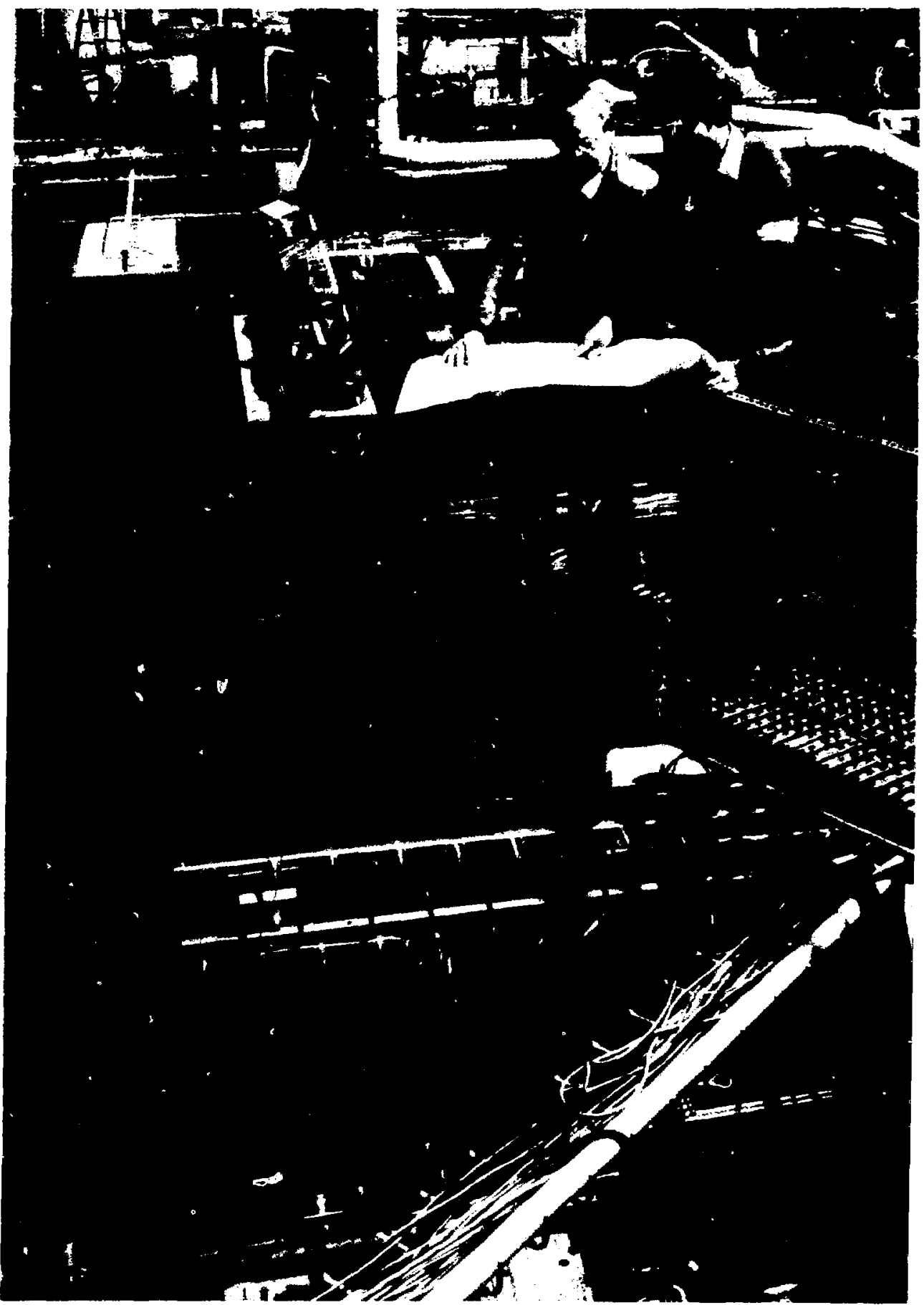

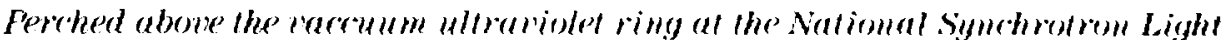

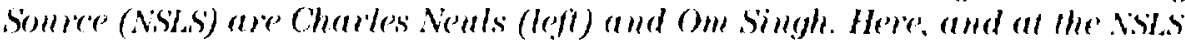

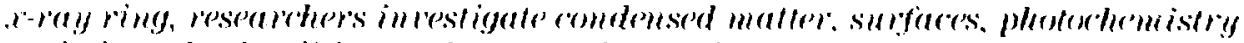
and photophysirs, lithography. argstallegyraphy. small-angle'scatlering and

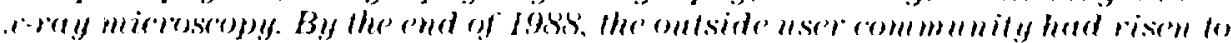

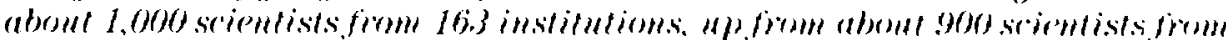

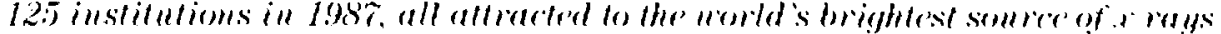
and ultarabiolet radiation. 


\section{High Flux Beam Reactor}

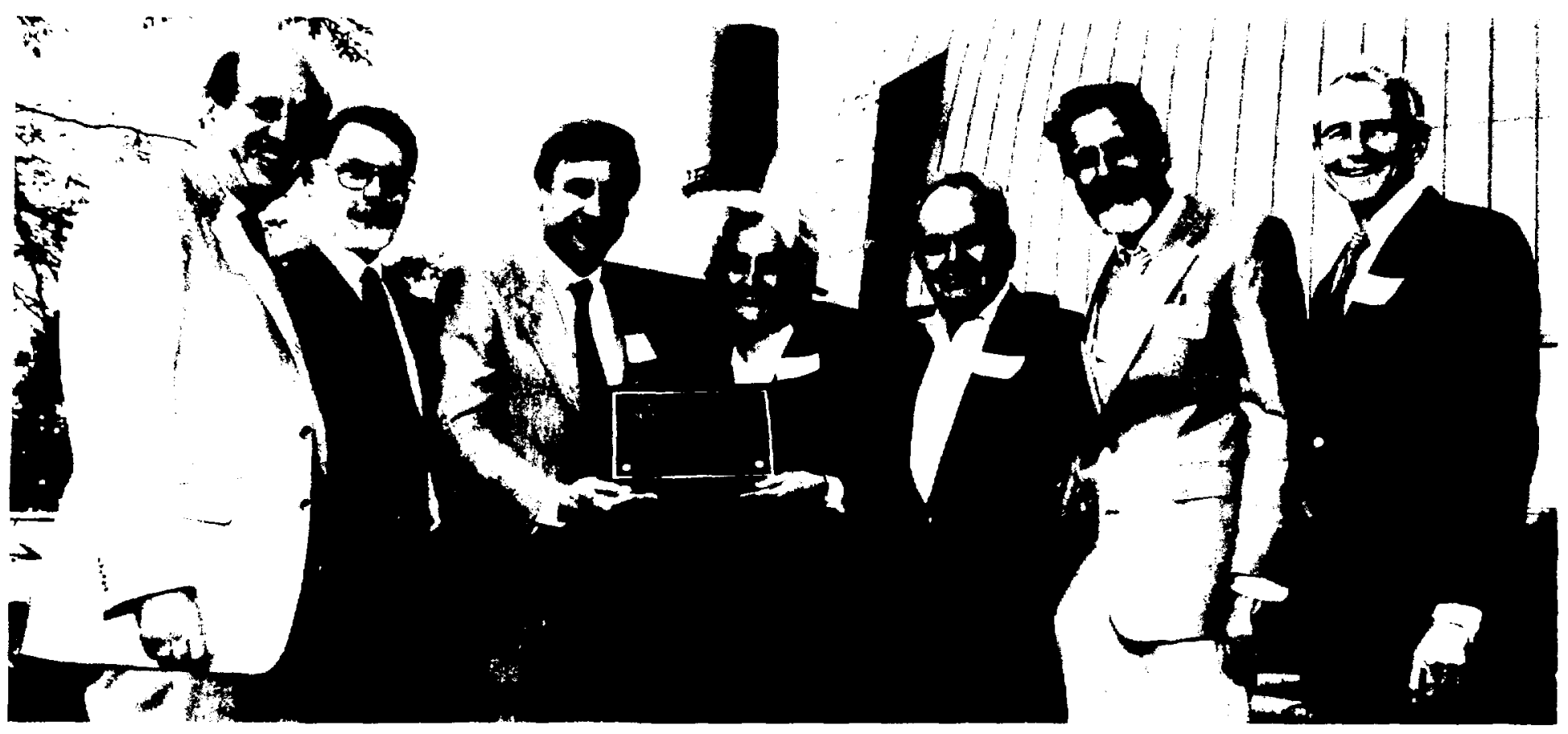

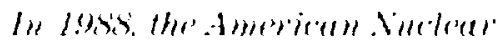

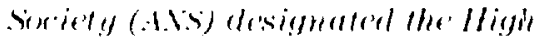

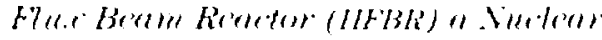

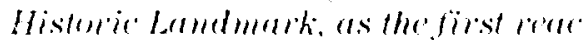

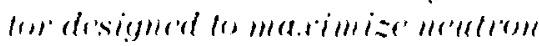

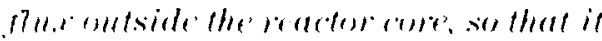

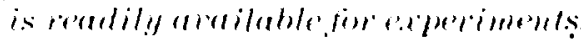

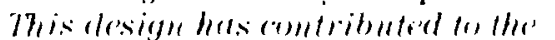
HFWh s reserd of high aroilabilit! and lemge assetid lite.

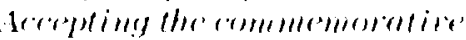

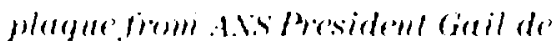

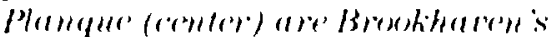

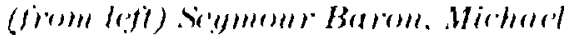

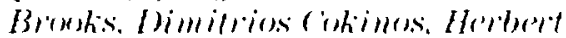

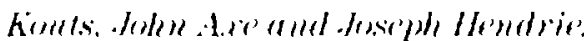

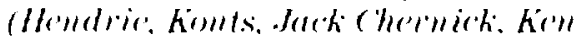

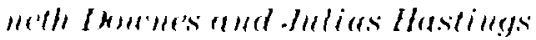

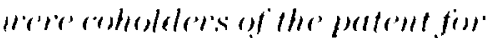
inremtimes the' HItsh.)

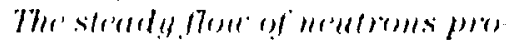

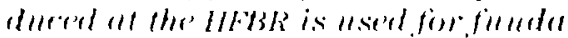

mental resistarth in solid state and

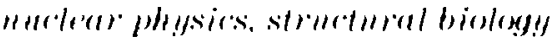

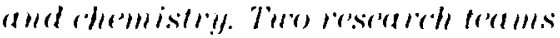

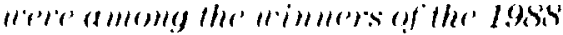

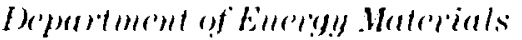

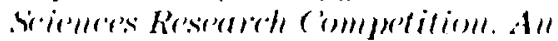

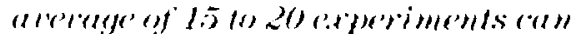

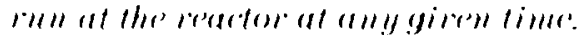

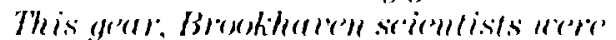

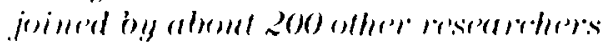
firem all arese the arold.

\section{Scanning Transmission Electron Microscope}

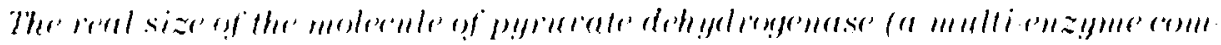

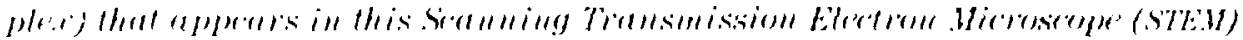

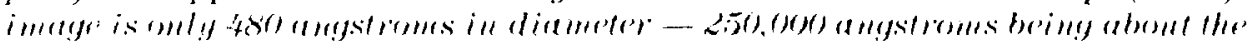

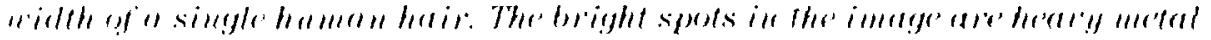

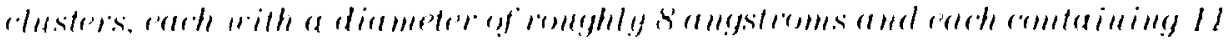

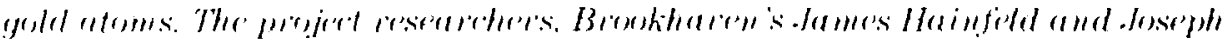

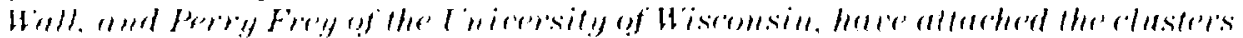

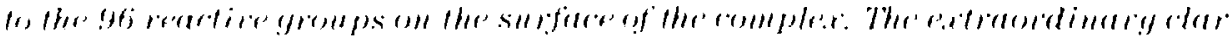

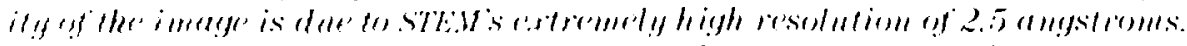

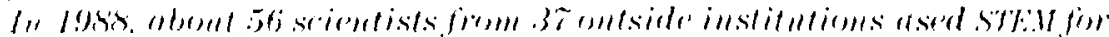

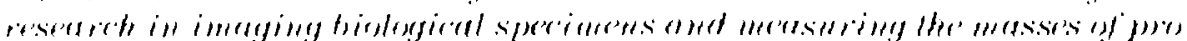

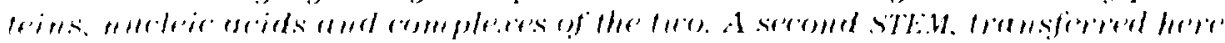

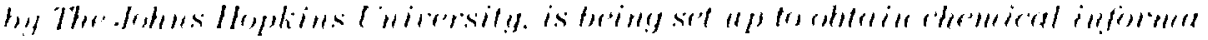

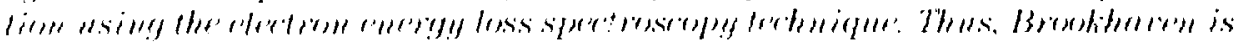

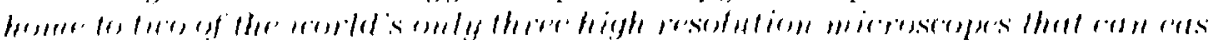

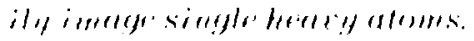

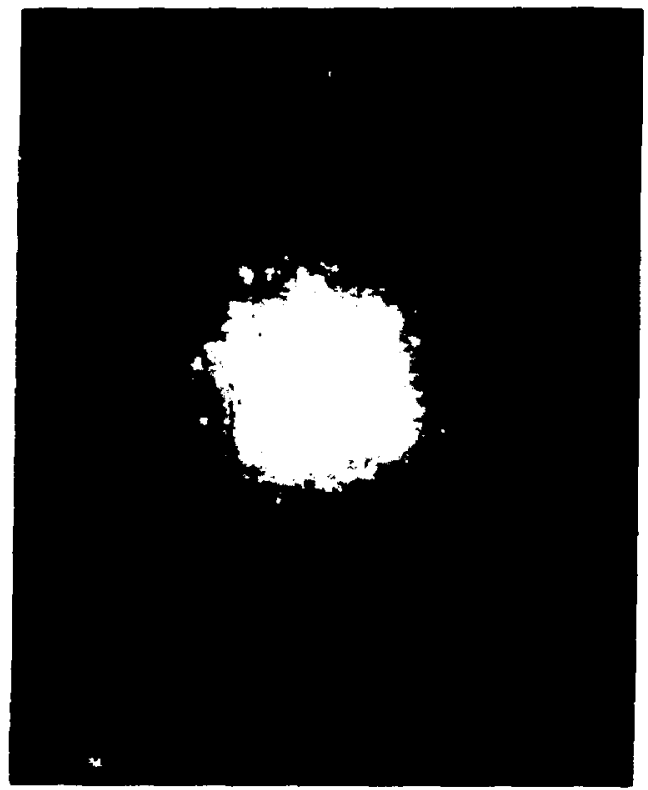




\section{Alternating Gradient Synchrotron}

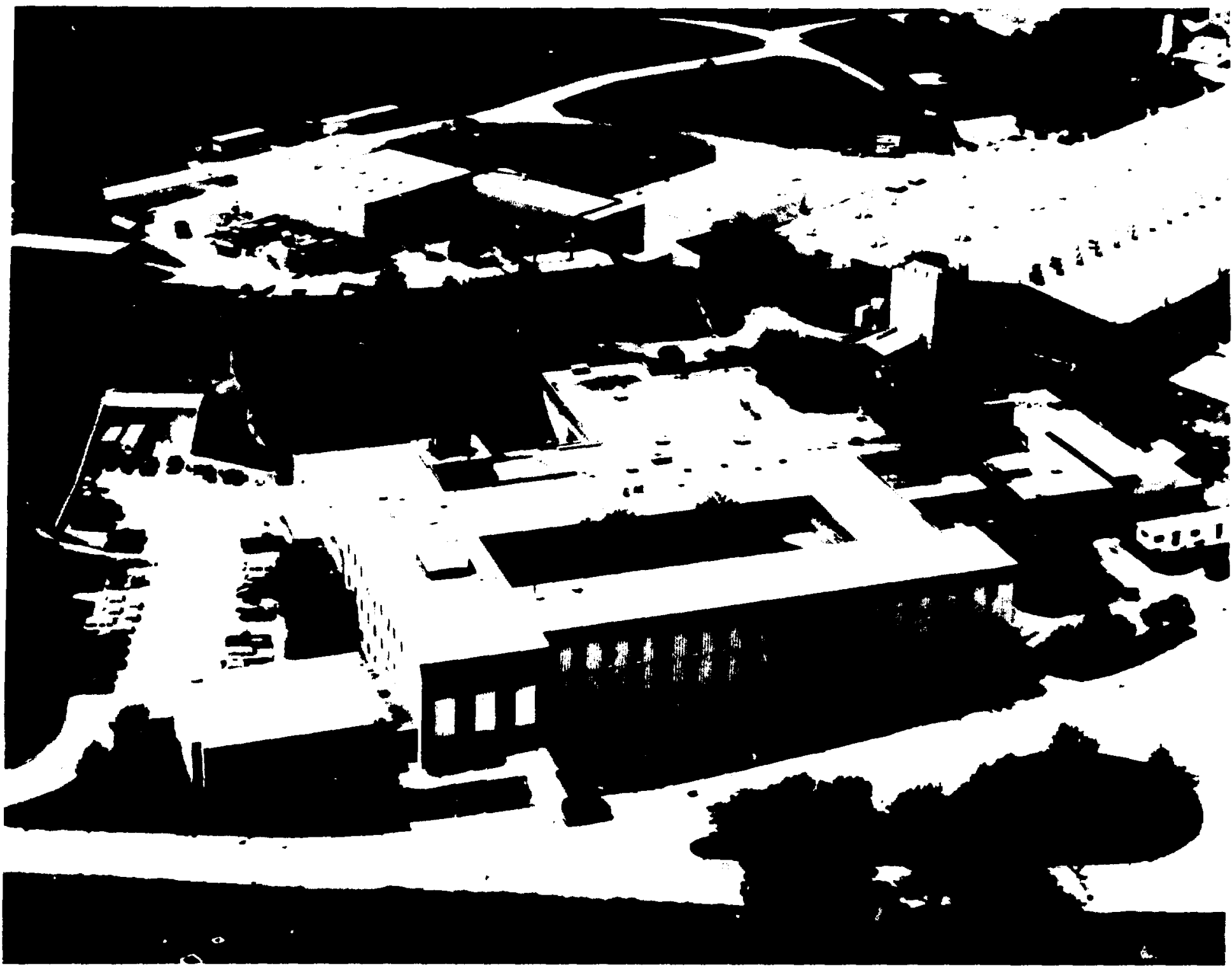

The importance of scientific research at the Alternating Gradient Symchrotron (AGs) is matked by three Vothel prizes in physsics, for the disconeries of the muon-nentrino, ('P riolation and the .I psi particle. together with other major discoveries such as the Omega-minus paticle and the first charmed baryon.

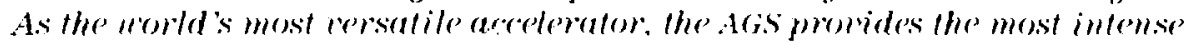

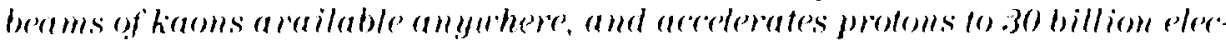

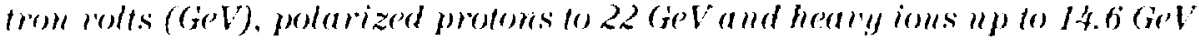

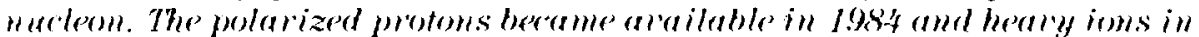

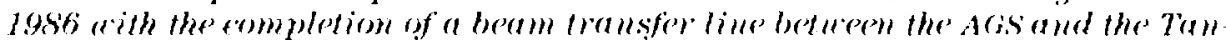

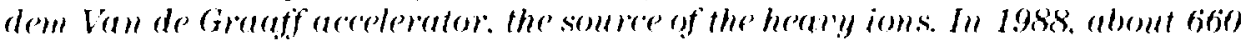

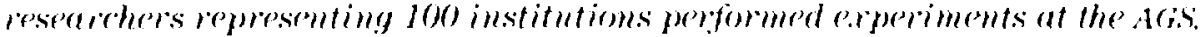




\section{Alternating Gradient Synchrotron Department}

The AGS Department operates the Alternating Gradient Synchrotron (AGS), the centerpiece of Brookhaven's high energy and nuclear physics program. In recent years, with the completion of a transfer tunnel between the Tandem Van de Graaff and the AGS, heavy ions have joined protons and polarized protons as the particles of choice for researchers who come from all over the world to do their experiments at the AGS. The latest project to get under way is construction of the AccumulatorBooster, which will greatly enhance both the nuclear and high energy physics programs at the Laboratory.
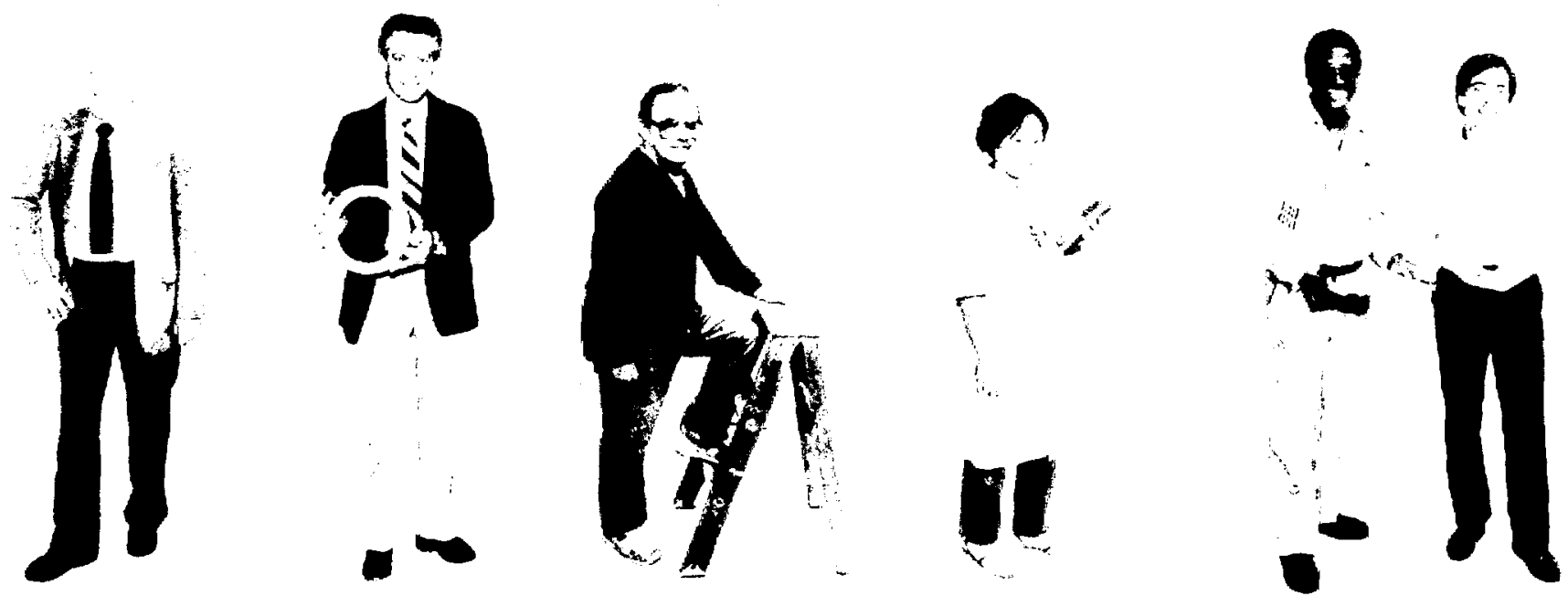


\section{Farewell, Cockcroft-Walton - Hail, New RFQ!}

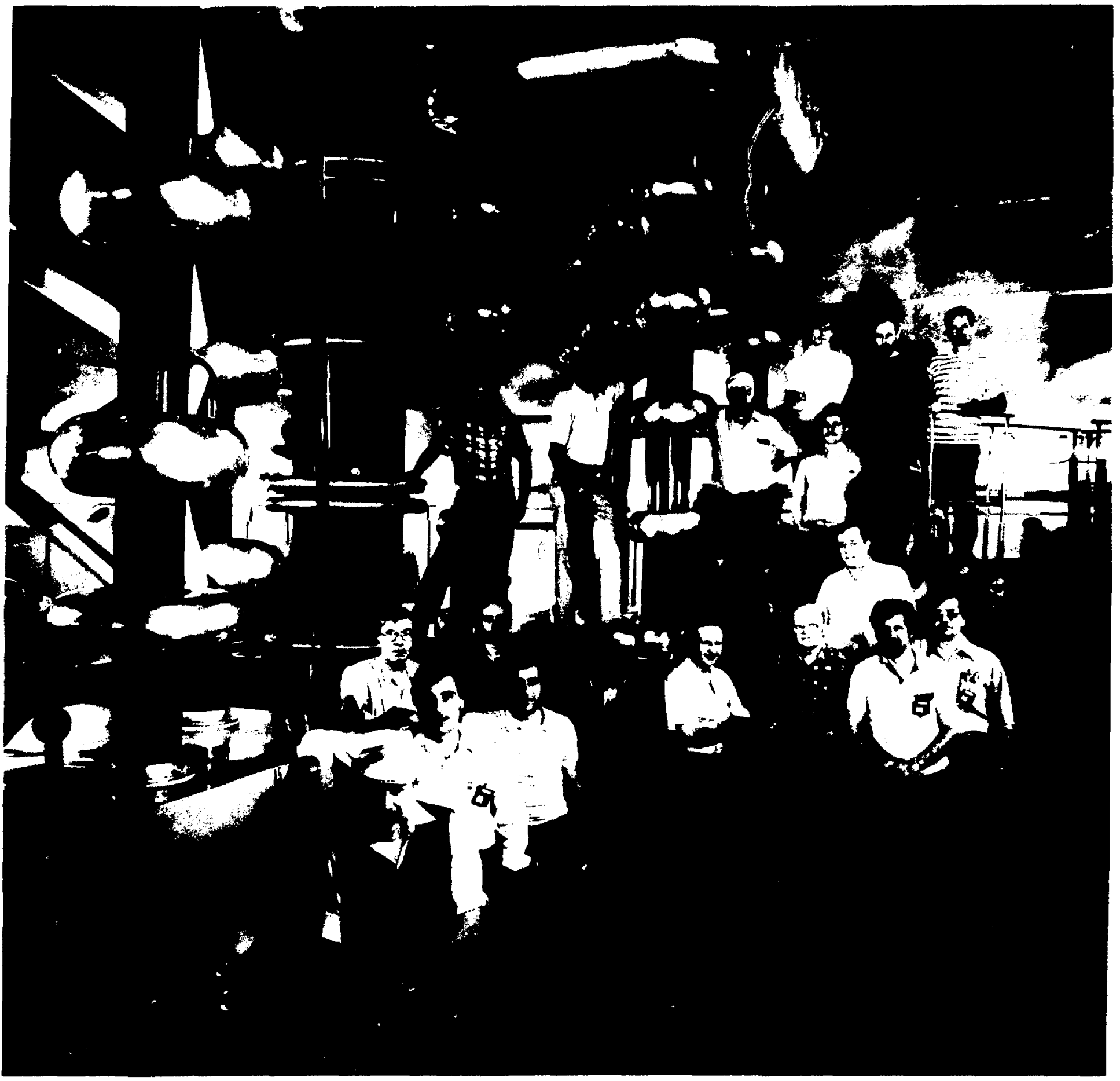

Involved in operating the Cockcroft-Walton preaccelerator were (fromt group, from left) Peter Montemurro (hatted), Walter Hersel, Vincent Lo Destro, Ronald Weider (back), James Alessi, Robert Glasmann, Jack Gandrey, Ahoi Kponou (back), Wermer Tramm, Charles Meitzler (bach), Brian Briscos. William Simmons, Walter Shaffer; (back group, from left) Ronald Clipperton (check shirt), John Brodowski, Vincent Konarik, Thomas Russi), Alfied Mack; (far back, from left) Daniel McCafferty, Frederick Usack and Gilbert De Gregory. 


\section{"The king is dead! Long live the} King!"

In history books, when an old order passes, the air resounds with the cries of acclain that welcome the new regime. In 1988, this sort of history repeated itself at the Alternating Gradient Synchrotron (AGS), when the mighty Cockcroft-Walton preaccelerator ended its time of service, ceding the path to its equally powerful successor, the radio frequency quadrupole linac ( $\mathrm{RFQ}$ ).

Cockcroft-Walton generators were first designed in about 1932. At Broukhaven, our Cockcroft-Walton's working life started on July 29 , 1960, when, at precisely 4:13 p.m., the first beam to circle the brandnew AGS was initiated. The next day, the New York Herald Tribune reported on the successful test of the "World's Most Powerful Atom Smasher." it described how the beam began in an electric sparking chamber where electrons were torn from hydrogen atoms to make bare hydrogen nuclei, or protons.

"Quickly the protons rippled into a futuristic two-story device called a Cockcroft-Walton generator. There, the protons' tiny energies were boosted to 750,000 electron volts, and the particles sped out into another device called a 'linac, or linear accelerator..."

The Tribune continued with a description of how the protons shot on from the long, straight linac into the AGs ring. There, guided on their course by the pull of 240 magnets, they circled faster and faster, their speed boosted by radio frequency cavities.

\section{In less than a full second, the} protons had circled the ring 300,000 times and were traveling at almost the speed of light, 186,000 miles per second. They then smashed into a target, thereby feeding the experimental equipment set up to study the resulting interactions.

Almost three decades have gone by since that day in 1960, and a

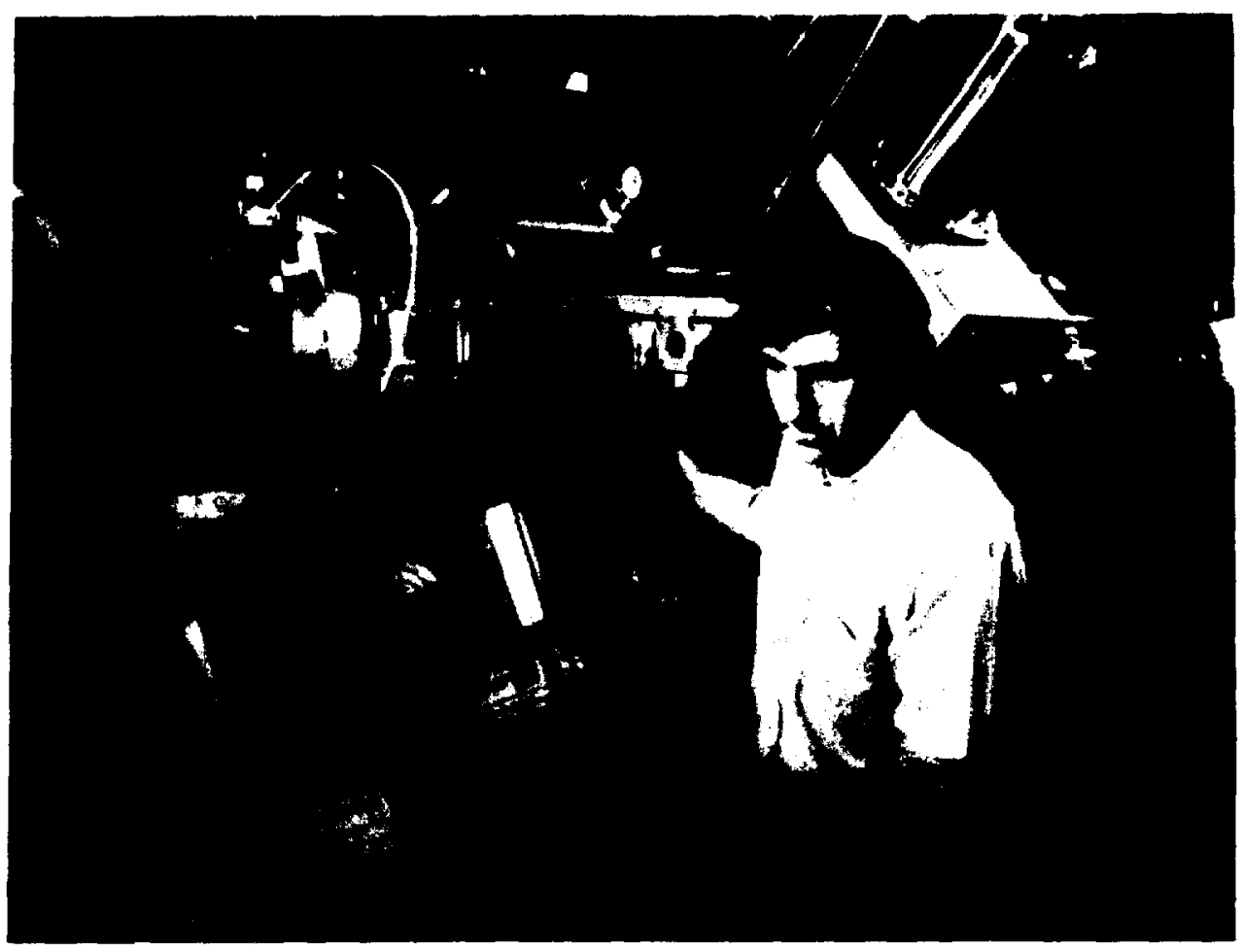

James Alessi, leader of the $R F Q$ II preaccelerator project, is standing in from of the mewly installed 750-keV RFQ II.

Cockcroft-Walton has boosted protons on their journey to Acis experiments in the same way ever since. Powered by 750,000 volts, the preaccelerator has been housed in a three-story-deep pit entirely lined with aluminum sheets. The pit must be dust-proof and humiditycontrolled to prevent lightning flashes from the high voltage.

Because of the Cockeroft-Walton's very high voltage, special safety measures and time-consuming maintenance were needed. These were the main reasons for its replacement by the $R F Q$. The new preaccelerator is only about 60 inches long by 18 inches in diameter and operates at normal, low voltages, so it needs no surrounding extra space. It is also much easier 10 maintain.

$\mathrm{RFQ}$ technology was introduced at the AGs in 1984, when a Brookhaven-designed $R F Q$ was the first in the world to accelerate polarized negative hydrogen ions, which entered the $\mathrm{RFQ}$ at 20 kilovolts (keV) and were accelerated to 750 kev before being injected into transport lines to the linac. This lirst $R F Q$ worked perfectly and proved to be very reliable.

Mostly made of copper-plated steel, an $R F Q$ is basically a tube that can accept a high-current, lowvelocity beam. Attached to the inside of the tube and running its length are four copper-plated steel vanes that project radially toward the center. The tips of the vanes are machined in a wave-like pattern. This causes the radio frequency electric fields in the RFQ to bunch. focus and accelerate particles to $750 \mathrm{keV}$ at the linac's frequency of 201.25 megahertz.

In its day, the Cockcroft-Walton preaccelerated the proton beams that made possible the Nobel Prizewinning discoveries of the muonneutrino, (P'violation and the J psi particle, as well as such ot her notable findings as the anti-xi-minus, the Omega-minus and the charmed baryon particles. The new RFQ preacelerator, the second to bo built at Brookhaven, promises to carry on this trastition of exriting physies at the $A$ cis 


\section{A Search for Eventful Physics}

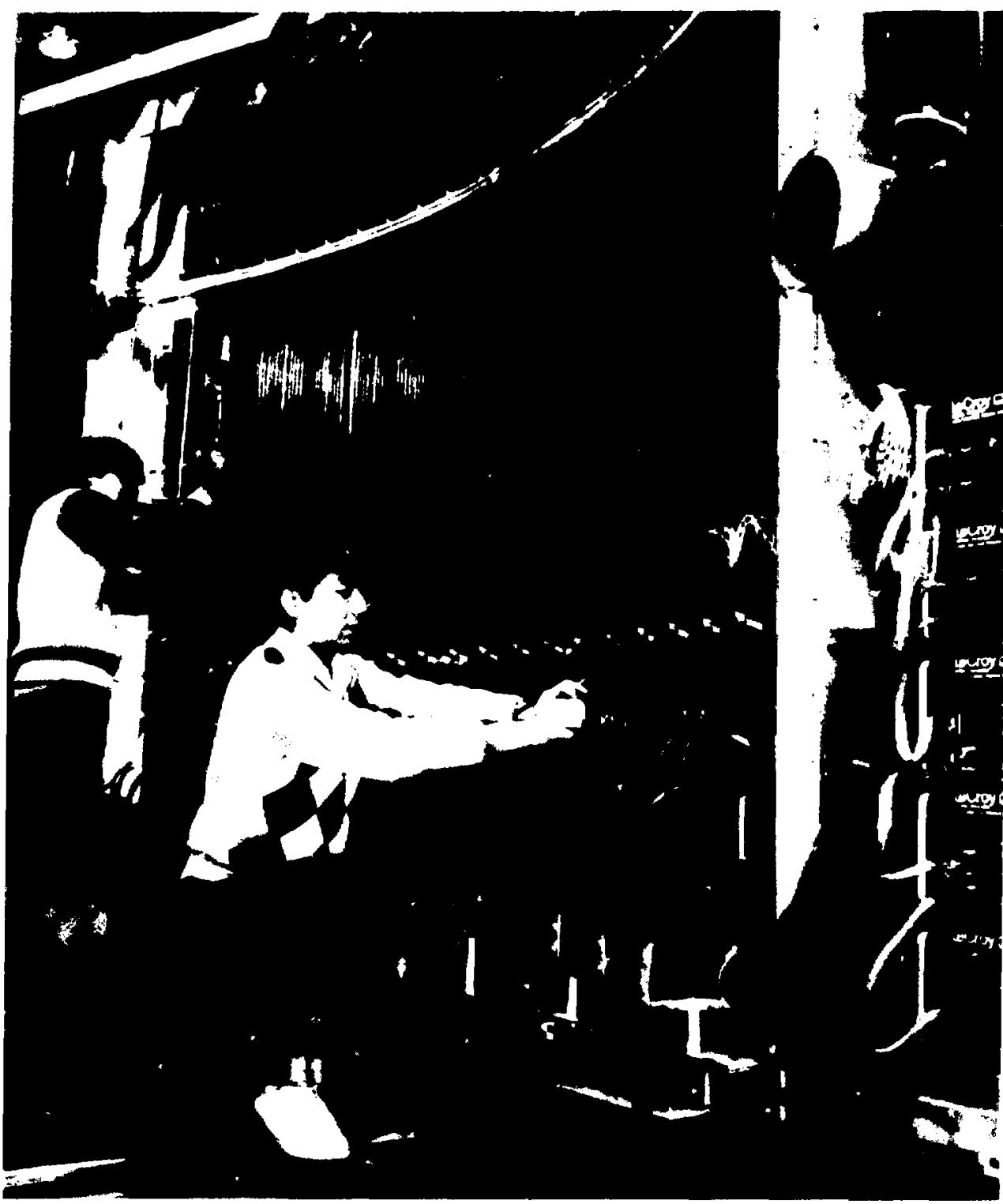

Broskhaten's Yasuo Miake and Shige Hayashi, from the Lniversity of Tokign, readjust wires in the time-offlight wall that they buill to enable the relority of particles in Experiment 802 to be measured.

$\mathrm{W}_{\mathrm{h}}$ the Alternating Gradient Synchrotron (AGS) is running, coimtless signals are pulsing through thousands of feet of cable to be scanned, sorted and recorded in a computer. Most of the clata generated form an expected patterr - but some stand out. These are the "interesting events" that call for analysis.

The 60 nuclear physicists at E802 are studying what happens to parti- cles formed at the extreme densities created in nuclear collisions. 'fo create these conditions, they smash heavy ions of oxygen or silicon into a fixed target.

A heasy ion is the nucleus of an atom, st ripped of its clect rons. Only since 1986 have heary jon experiments been possible at Brookhaven's Acis. In these oxperiments the search is on for now states of nulclear matter noser before observed.
The Brookhaven hoaty jon slory stanted in lobo when the Tandem

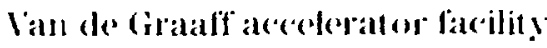
was proposed for the study of muclear physics. It begall operat ion in 1970 and quickly became llec world's most powerful heasy ion laceility. used by researchers fiom many nations. In 198:3. Brookhaven physiceists first developed a highcurrent, pulsed souree of negatives jons and showed that pulsed current could be accelerated by the Tandem. Then, the remaining question was how to tlansport the beam from the Tandem to the Acis.

This was done by modifying both facilit ies. Since the beam has to be injeceterl into the Acis in short, highrurtent bursts, each carefully timed. the control fo: the Tandem solsed negat ive ion sourees was modified to allow for AGs synchroniyation.

Then, 10 comnect the Tandem to the Acis, a 2,000 -foot-long tumnel, ten foet in diametor, was built. A computer-cont rolled boam transport system cousisting of over a hundred magnets was inst alled to guide the heary ion beam on its journey to the Alis:

At the Acis it self, a inew beam injection system, a new computer system and an additional radio fiequency acceleration cavity were inst alled. Wit h these improvements, the heary ions coming in from the Jandem at an energy of $7-8$ million electron volts per nucleon ( MeV II) can first be accelerated in the Acis to $200 \mathrm{Mel} \mathrm{n}$, where the existing accelerating system can then spored them up to $14,600 \mathrm{Mel} n$.

In 1986, when the Acis lirst aceelelated a beam of oxigen ions 10 $14,600 \mathrm{Mel} \cdot \mathrm{n}, \mathrm{E} 802 \mathrm{2}$ was ready and watiting for it - one of the fint major heary ion experiments to get under way. When the experiment is runnings, the beam smashes into the target, and particles ny out in all direcetions at close to the speesl of light. Fareh part of the (romomous ger nlt rasensitive \& tus ejt hor measures glohal proper ties of the int eraction, suelo as the 


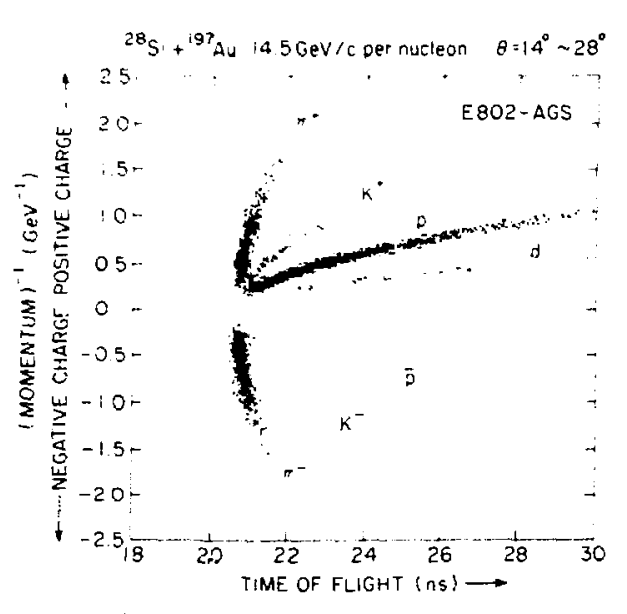

Particles are identified by combining the momentum analysis and the timeof-flight information. The scatter plot shou's the correlation between time of flight and the inverse of particle momentum for silicon-plus-gold collisions. The assigned particle identifications are indicated on the plot.

energy flow at each angle; or individual properties of the emitted particles, such as their momentum.

One way particles are distinguished from each other is by their mass, which can be obtained by measuring their velocity and momentum.

In E802, to get the velocity, a beam counter records the instant that a particle strikes the target. Then, when the particle reaches what is called the "time of flight wall," the time is recorded again. The time taken from the target to the wall is called the particle's "time of flight" and is measured to a precision of one-ten billionth of a second. The measured time of flight and the known path length gives the particle's velocity.

So that their momentum can be measured, after hitting the target, and before the time of tlight wall, the particles pass through "Henry Higgins," a huge magnet that has played a key role in many important physics experiments in the past. Both before and after they pass through the magnet, their position is measured to one-hundredth of an inch in a series of devices called drift chambers. From the positions measured in the drift chambers, the angle through which a particle bends as it passes through the magnet can be determined, and, in turn, this angle determines the particle's momentum.

Computer-produced graphs can then be made using each particle's momentum and velocity together to show its mass (see figure), and, thus, its identity.

The particles pass through other finely tuned instruments, such as a lead glass array that detects energy from neutral meson particles, and a calorimeter that measures how many nucleons of the incoming ion pass right through it without collid- ing. Then all the dat a are assembled by a central computer and studied at length by the E802 physicists.

Results so far have produced at least two surprises. One is that the calorimeters measuring transverse energy record the same maximum whether the target is a gold nucleus or a copper nucleus, which is only half the size of the gold. This means that the oxygen nucleus can be completely stopped in either of these target nuclei, thus adding to our present information on nuclear stopping power and indicating that regions of high density can be formed in these collisions.

Also, when silicon nuclei collide with gold at a certain energy, the ratio of positive kaons to positive pions produced in the interaction is twice as large as that produced when protons, which are hydrogen nuclei, collide at the same energy. This was not predicted by the theoretical models of nucleus-on-nucleus collisions based on data from proton-on-proton collisions. So far, this result remains unexplained and that makes it atl the more interesting. The outcome may throw new light on the high-density, hightemperature environment created in heavy ion collisions.

\section{Future Plans}

The current AGS heavy ion program is expanding, and more facilities are on the way.

In September 1987, ground was formally broken for the AGS Accumulator-Booster. The small, powerful Booster, projected for completion in 1991, will preaccelerate all species of heavy ions journeying from the Tandem to the AGs, as well as increase the intensity of protons for other AGs experiments.

At present, only medium mass ions, up to sulfur (atomic mass 32 ), can be accelerated in the AGS. With the additional energy from the Booster, however, the AGS will be able to handle the whole range of heavy ions, from carbon (atomic mass 12) all the way to gold (atomic mass 197).

Brookhaven's future planners see heavy ions as traveling beyond the AGS, into the proposed Relativistic Heavy Ion Collider. There, the collision of two beams of heavy ions, at a combined energy of 200 billion electron volts, would allow physicists to study matter as it was at the first millionth of a second of the "Big Bang" when the universe was born. 


\section{Forbidden Violations}

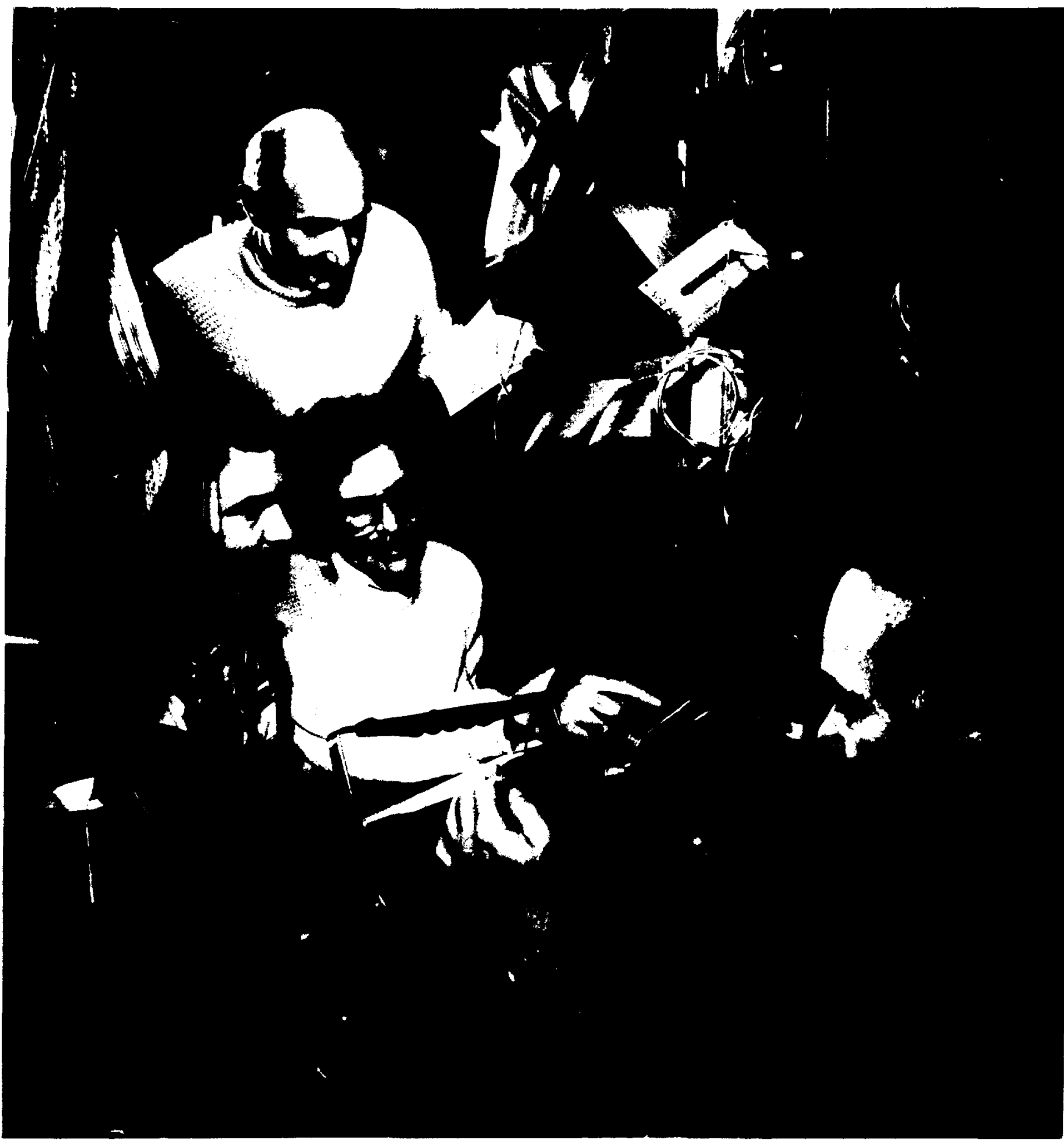

Pavel Rehak (top) and (from left) James Mills, Howard Gordom and Donald Lazarus are checking the timing of the strobe of the multivire proportional chambers in the Experiment 777 apperatus. 
Nobody has ever observed a violation of lepton generation number that 's the very reason physicists are looking for one. A likely place to search for such a violation is in unusual decavs of kaon $(K)$ particles. which explains the intense interest in the rare $\mathrm{K}$ decay experiments at Brookhaven's Alternating Gradient Synchrotron (AGS), the world's most intense source of kaons.

If a violation is found, the discovery will create problems for what has become known to particle physicists as the standard model (the way matter is believed to be formed), and a window may open into entirely new physics.

What, exactly, is "lepton generation number violation"? To understand the term, one must know something about the standard model, which has been enormously successful in its agreement with experiments.

The knowni elementary particles can be divided into three families: quarks, leptons and the gauge bosons through which they interact. The lepton and quark families are formed of six indivisible particles (and their antiparticles), and each group of six is paired into three generations of two kinds, or flavors, of particles (see chart).

The standard model incorporates lepton generation number conservation - the numbers assigned to the leptons of each generation must be conserved in any allowed process.

For example, a negative muon, which is in the second generation of the lepton family, cannot decay solely into an electron, which is in the first generation. It can decay into a muon neutrino (second generation), an electron and an electron antineutrino. This is because the first generation particle (the electron) and the first generation antiparticle (the electron antineutrino) cancel each other, leaving one second generation lepton (the muon neutrino) to correspond with the second generation lepton (the muon) that started the whole process. Thus, the lepton family number does not change in this decay.

Armed with the sophisticated detector techniques available today, physicists at the AGS are marshaling their forces to explore this area in a new series of kaon decay experiments. Three of the experiments are searching for $\mathrm{K}$ decay modes that are forbidden due to lepton family number violation. Also being investigated are some modes that are predicted to occur although they are highly suppressed in the standard model.

Experiment 777 (E777) is one of those whose object is to spot the forbidden. This is the longestrunning rare $\mathrm{K}$ decay experiment, having first collected data in February 1985 . If the researchers observe a positively charged kaon decaying into a positive pion, $\pi^{*}$, a positive muon, $\mu^{+}$, and a negative electron $\mathrm{e}^{-}$, they will have evidence for lepton family number violation.

To recognize that the decay $\mathrm{K}^{+} \rightarrow \pi^{+} \mu^{+} \mathrm{e}^{-}$has occurred, the researchers must detect an electron coursing through their experiment's apparatus, which is a very sensitive spectrometer with excellent particle identification properties. For good data, the experiment must be able to differentiate an electron from a pion with virtually no errors.

To make the apparat us that sensitive, three detectors - two Cerenkov counters and a lead scintillator shower counter - are used in series to identify the negatively charged electrons, which are magnetically deflected to the left side of the experiment. If all three detectors recognize a particle as an electron, then it probably is one - with a misidentification occuring at most only once in any ten million events.

Similarly, the Cerenkov and shower counters on the right side discriminate against posit rons (antielectrons) and guarantee that the positively charged particles are pions and muons, which are distinguishable by their range prior to stopping in a stack of steel plates interspersed with particle detectors.

By 1987, ten billion positive kaons had passed through E777. One billion of the kaons decayed, and not one showed a violation of lepton family number. In 1988 , after another ten billion decays, there was still no sign of the positive pion, positive muon and negative electron combination, although the data are still being analyzed. If no signal is found, it will be possible to establish an upper limit on the probability of positive kaons decaying in this

\begin{tabular}{|c|c|c|c|}
\hline \multicolumn{4}{|c|}{ Quark \& Lepton Families } \\
\hline Generations & 1 & 11 & III \\
\hline${ }^{*}$ Quark Family & $\begin{array}{l}\text { up } \\
\text { down }\end{array}$ & $\begin{array}{l}\text { charm } \\
\text { strange }\end{array}$ & $\begin{array}{l}\text { * top } \\
\text { bottom }\end{array}$ \\
\hline `Lepton Family & $\begin{array}{l}\text { electron (e) } \\
\text { electron- } \\
\text { neutrino } \\
\left(v_{e}\right)\end{array}$ & $\begin{array}{l}\text { muon }(\mu) \\
\text { muon- } \\
\text { neutrino } \\
\left(\nu_{\mu}\right)\end{array}$ & $\begin{array}{l}\operatorname{tau}(\tau) \\
\text { tau- } \\
\text { neutrino } \\
\left(\nu_{\imath}\right)\end{array}$ \\
\hline $\begin{array}{l}\text { *All quarks an } \\
\text { * } \text { Postulated, b }\end{array}$ & $\begin{array}{l}\text { have anti } \\
\text { t discovere }\end{array}$ & unterpar & \\
\hline
\end{tabular}




\section{The Unseen Axions}

While E7T7 researchers look at so many $K$ decays, they are able to search for other decays. For example, theoretical physicists have proposed the existence of a light, short-lived particle known as an axion.

Axions are among the leading candidates to explain the missing mass of the universe, or what is known as "dark matter." In 1933, astronomer Fritz Zwicky analyzed photographs of the Coma galactic cluster: Discovering that the galaxies were being attracted together by a gravitational force much stronger than the mass of their stars could generate, he proposed that 90 percent of the matter in galaxy clusters is invisible.

Axions have never been observed. If they can be proved to exist. they may not only explain "dark matter," but also, other parts of the standard model that are not yet fully understood.

Among the decay modes available to axions is one that results in an electron and a positron.
Experiments in collisions of heavy ions at Gsi in Darmstadt. West Germany, have shown correlated electron-posit ron pairs that are consistent with the decay of light neut ral particles that are possibly of axion or axion variant parentage (see related story page 30 ).

Members of E777 searched for a positive kaon decaying into a positive pion and another, neutral. weakly interacting light particle. This particle, whose mass would be similar to that implied by the Gsi results, would itself very quickly decay into a positive electron and a negative electron.

The results, which complement those of previous searches elsewhere, show that finding such a particle through this $\mathrm{K}$ decay is unlikely - less than 4.5 times in ten million. And even this small likelihood depends on the axion's lifetime being short enough for it to decay before passing through the experiment - a single tenmillion-millionth of a second! mode. In ot her words, the probability of a kaon violating lepton generation number in this way is likely to be less than one in ten thousand million.

For the present, therefore, E777 has not presented a challenge to the standard model. 


\section{Accelerator Development Department}

The Accelerator Development Department (ADD) plays a key role in designing and building the new generation of high energy and nuclear particle accelerators at the Laboratory. Thus, ADD consolidates Brookhaven's activities in the construction of the Accumulator-Booster for the Alternating Gradient Synchrotron and in the technical development of the Laboratory's proposed Relativistic Heavy Ion Collider. The department is also responsible for BNL's contribution to the nationally proposed Superconducting Super Collider.
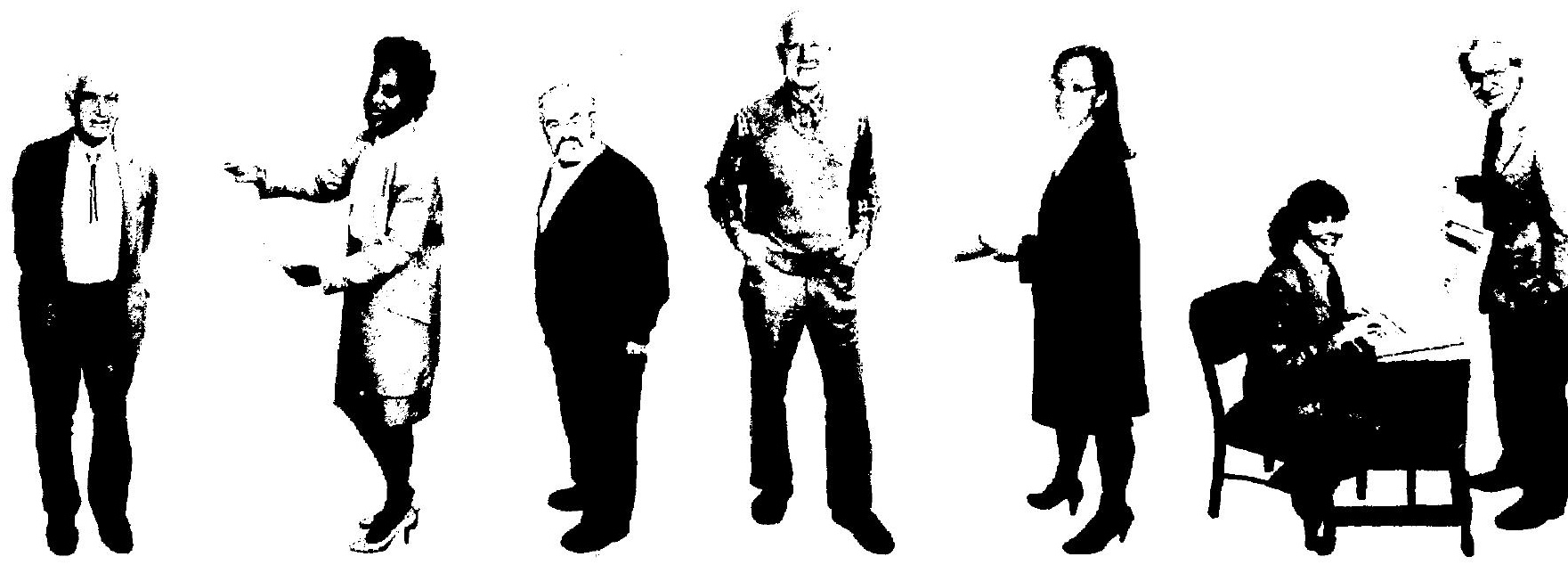


\section{High Marks for the Booster}

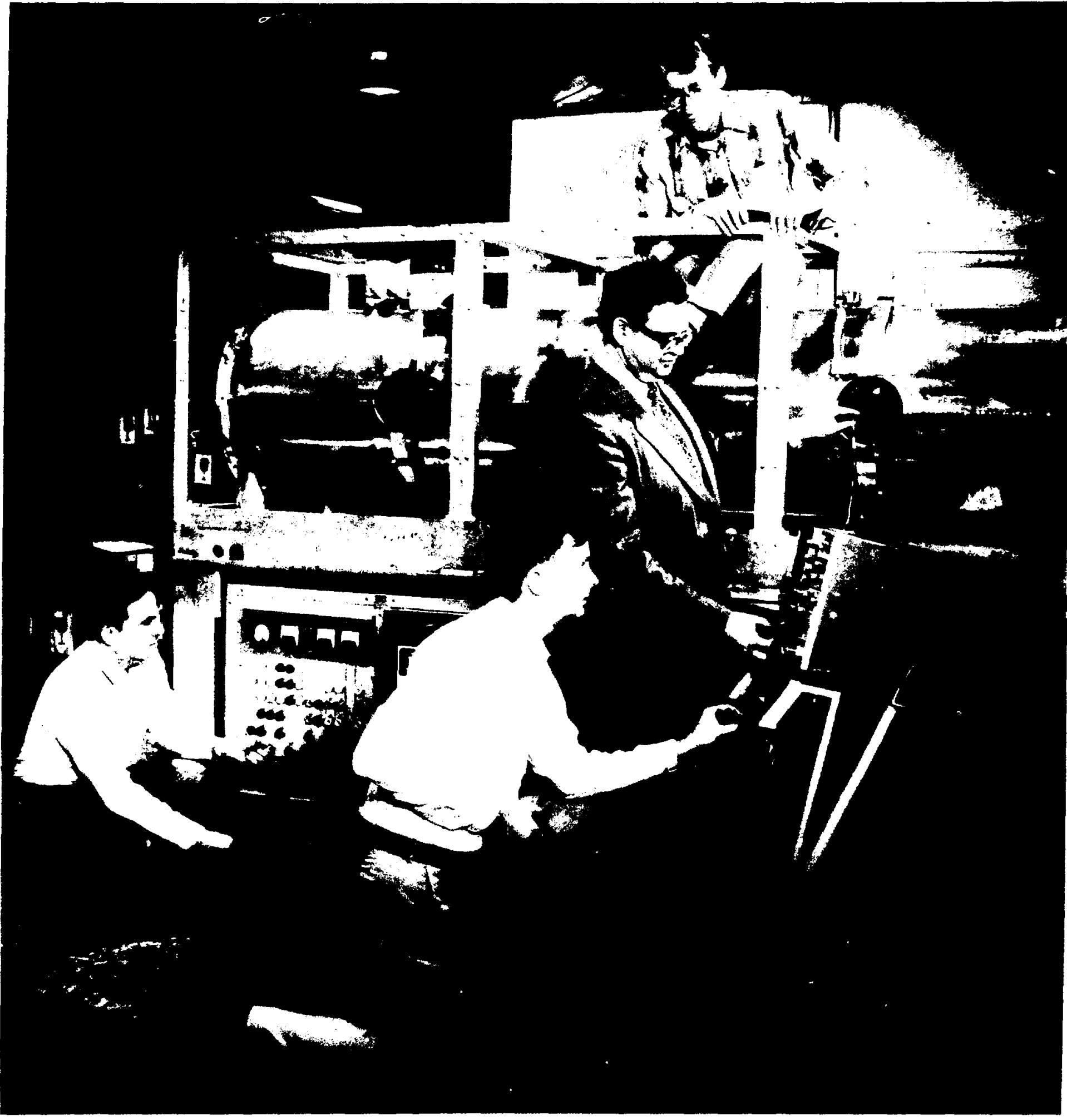

Broskhaven engineers (from inf) Philip Stattel, Ralph Sianders, Mirhad Goldman ard Peter Cameron test prototypess of a radiof frequemey racity and pourer aimplifier for the Booster: 


\section{Brookhaven's Accumulator-}

Booster Project received high marks during a review by the L.S. Department of Energy (DOE) in mid-1988.

Projected for completion in 1991 , the Booster will be a small accelerator that will give particles an intermediate phase of acceleration before entering the Alternating Gradient Synchrotron (AGS). The Booster will increase the intensity of protons and polarized protons from the AGS linac, for high energy physics experiments, as well as allow the AGS to accelerate the whole range of heavy ions generated in the Tandem Van de Graaff accelerator. This latter task is an essential step in the acceleration of heavy ions in RHIC, the Relativistic Heavy Ion Collider that Brookhaven has proposed to build.

The DOE review of the Booster project covered technical design, construction, cost, scheduling and management. Among the technical achievements cited by DOE was a prototype half-cell - a complex of dipole, quadrupole and sextupole magnets, together with a vacuum chamber and ion pump, all mounted on a common base placed in a mock tunnel. The purpose of building the half-cell was to test the effect of space restrictions, alignment of components, installation of the beam tube and performance of vacuum and electrical equipment.

Following the DOE review, notable progress continued in three major areas.

\section{Conventional Construction}

Even though ceremonial groundbreaking for the Booster occurred in September 1987, actual tunnel construction began only in March of 1988. By the end of the fiscal year, however, most of the tunnel enclosure had been completed and work begun on concrete structures that will tie the Booster to existing facilities.

Viewed from the air, the Booster looks like a doughnut sitting

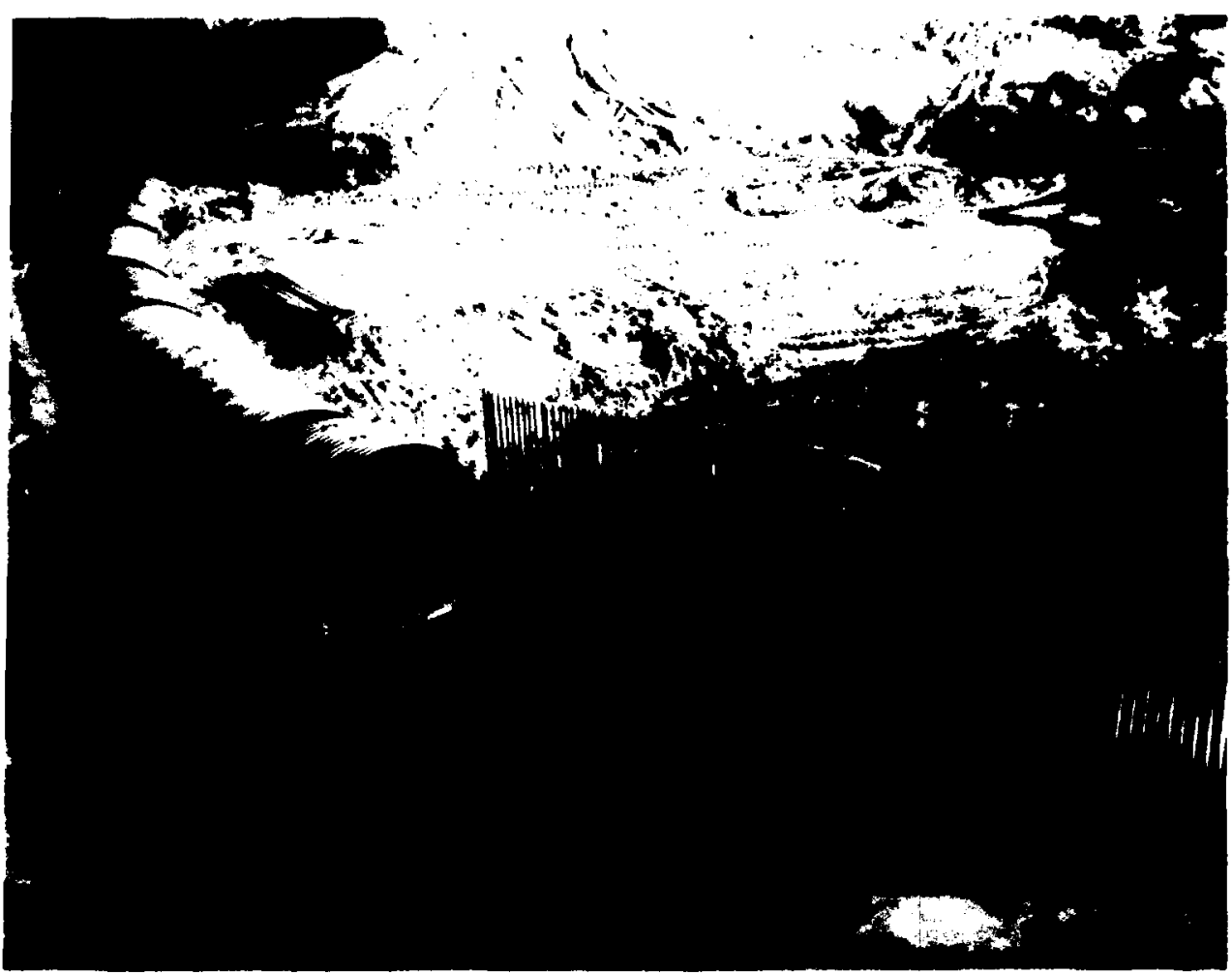

Conventional construction for the Booster tunnel began in March of 1988. The tunnel is made of a cormigated steel pipe, 11 feet in diameter.

between the linac and the AGS. The tunnel itself is an 11-foot diameter corrugated steel pipe, buried under 15 feet of earthen sitielding.

It was crucial that the penetrations into the existing facilities be completed during the AGS summer shutdown, a three-month period, so as not to interfere with the normal AGS operating schedule. As soon as the accelerator was turned off in early July, bulldozers began to remove the earthen shield over sections of the AGS and the linac. The facilities were penetrated and a great deal of concriete work was done before the earthen shield was put back.

As a result, the Booster project is on schedule, and in the spring of 1989, the Laboratory expects to be able to begin installing accelerator components. All services to the facility, including power, lights, air and water, will be completed by the end of the summer.
Overseeing the conventional construction is the project coordinatior group within Brookhaven's Plant Engineering Division.

\section{Shielding Blocks}

Also vital to keeping the project on schedule so as to accommodate AGS operations was work done by the AGS experimental areas group, which regularly works on shielding for AGS experiments. For the

Booster project, they were asked to design, fabricate and install shielding between the Booster and the AGS. Essentially, what was needed was a radiation barrier so that people could safely work in the Booster area with the AGs running. The time constraints were the same: all work had to be completed during the AGs shutdown.

The job was done with about $7(0)$ tons of shielding, which is made up of a combination of standard con- 
crete block and high density concrete block that contains an iron ore called ilmenite. Most of the AGs blocks use ilmenite, which has to be shipped down from Canada. A novel idea was proposed: Crush old AGS blocks to recover the ilmenite. The idea was tried and it worked. Because the ilmenite is extremely hard, when the concrete was crushed, the ilmenite was left behind. Then it was delivered to a concrete manufacturer, who simply added concrete to make new blocks.

\section{RF Systems}

In order for the Booster to handle both high intensity protons and a wide range of heavy ion species, it needs a sophisticated acceleration system. Critical to this system are ferrite-filled radio frequency (rf) cavities, which will bunch particles together and accelerate them to higher energies.

The AGs Department's Booster if group is in charge of designing and building the if systems. The Booster actually requires four $r$ systems. Two will be used specifically for accelerating protons; all four will be needed to accelerate heavy ions.

The two proton systems will be the most powerful ever designed at Brookhaven and, in their frequency range, will likely be the most power- ful in the world. The challenge presented by the proton systems called for the const ruction of a prototype. which was accomplished over the last half of the year. Next will come a comprehensive series of tests, which should verify the design.

\section{An Experiment With a Ring to It}

In In 1984, a workshop at Brookhaven drew dozens of physicists from the United States and Europe to discuss the feasibility of doing a single experiment. In the few years that followed, a formal collaboration was established, funding obtained and design work begun on the experimental hardware. Construction is to begin in the fall of 1989 , and the experiment will get under way at the Laboratory's Alternating Gradient Synchrotron (AGS) in the early 1990's.

What is this experiment that has attracted so much interest from the physics community? It is known as the muon g-2 experiment, and it represents a new kind of physics at the AGS, one that calls for a substantial investment in the construction of a giant superconducting magnet, built with the combined expertise of the Accelerator Development Department (ADD) and the AGS Department.

The thrust of the experiment is to make an extremely precise measurement of the muon's size, to see whether it has structure. Previous measurements of the magnetic moment of the muon have served as important tests of QED (quantum electrodynamics), the theory of electricity and magnetism.

The experiment has been done before at CERN, the European particle accelerator facility. How will it be done better at the AGS? Improvements in the intensity of the AGS beam over the years, plus the Accumulator-Booster now under construction, should result in an increase in intensity by a factor of 100 over the CERN experiment. That, together with the use of a specially built superconducting storage ring, will enable researchers to do the experiment 20 times more precisely.

During the past year, effort on this experiment, which has been assigned the number 821 at the AGS, has focused on the design of the storage ring. Actually one large superconducting magnet, the ring will be 14 meters in diameter and consist of sections of steel, four superconducting coils, a coil enclosure and lots of superinsulation.

The ring will be built in the same building that once housed the Laboratory's 80 -inch bubble chamber, a particle detector that was decomissioned in 1974. Because of limited space, a coil winding machine will be constructed in the building and the four coils wound there. Then the coils will be moved out of the building and the winding machine disassembled. Finally, the steel will be installed and the coils moved back in.

The superconductor is made of niobium-titanium, which is formed in a matrix with copper and the entire package thermally stabilized in a high-purity aluminum jacket. The aluminum reduces the possibility of the magnet quenching (losing its superconductivity when it warms). The coil design was heavily influenced by BNL's Japanese collaborators, and the superconductor is being supplied by the KEK Laboratory in Japan.

Quenching can occur when areas of the magnet are heated, usually due to movement of the magnet when it is powered. Since aluminum diffuses heat quickly, the quenching temperature is not likely to be reached. In lact, quenching should not be a serious problem because the magnetic field will be low (1.5) teslas) and the conductor is well- 


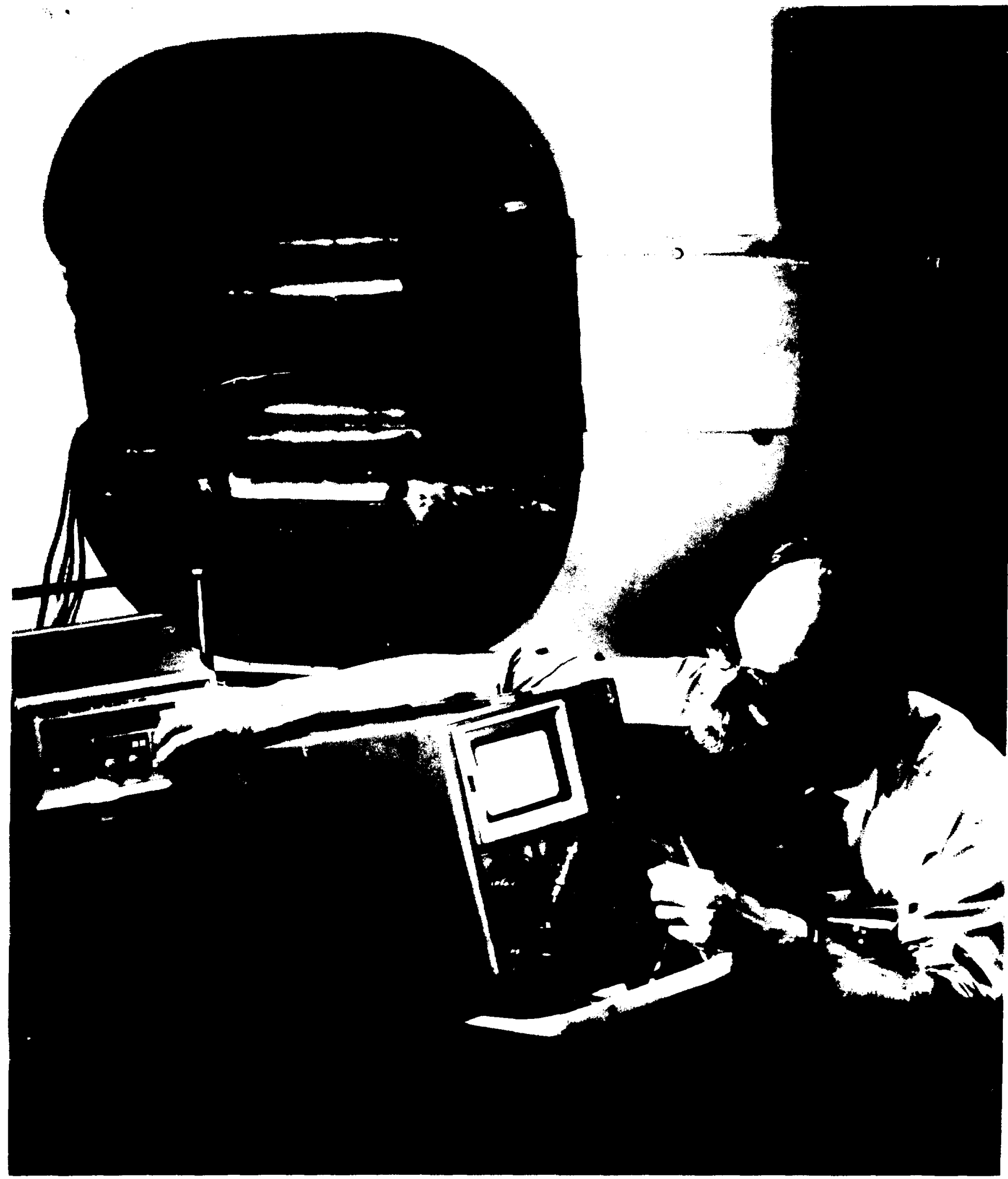




\section{Under the Big Top - The Physics of Muon g-2}

Under the big top, the peanut vendors are passing through the aisles, the band is oompahpahing, and all eyes are fixed on the graceful lady in pink doing acrobatic stunts on the back of a big white horse trotting gently around the ring.

In this circus scene, place yourself in the center of the ring where the ringmaster would be. Now watch the lady on the horse, who is doing a pirouette as the hor'se circles the ring. Note that she is turning on her own axis as she is carried in rotation around the ring.

In essence, the muon behaves in a magnetic field like the lady on the horse going around the ring.

The goal of the muon g-2 experiment, or E821 as it is known at the Alternating Gradient Synchrotron, is to get a more precise number for $g$ - 2 . The g stands for the muon's gyromagnetic ratio, which, according to an early theory of physicist Paul Dirac, should be equal to 2 . This would be true if the spin of the muon precisely tracks its change of direction in a magnetic field. In fact, it does not; the muon snin.s faster on its own axis, just as the lady on the horse is pirouetting faster than she is rotating about the ring.
The muon deviates from this precise tracking by one part in one thousand, and this deviation is understood exquisitely well to a few parts in a million $\left(10^{6}\right)$ by the more modern electroweak theory, which combines the weak and electromagnetic forces into a single theoretical framework. E821 will put this theory to a severe test, measuring the deviation to three parts in $10^{7}$.

The experiment will proceed as follows: Protons from the AGS will produce pions, which will then d or to muons. The muons will be trapped in a giant magnetic ring, where the direction of the muon spin will be observed. stabilized due to the large volume of aluminum.

The superconductor will be cooled in two stages: first to 77 kelvins $\left(-320^{\circ} \mathrm{F}\right)$ with liquid nitrogen, then to 4.5 kelvins $\left(-451^{\circ} \mathrm{F}\right)$ with liquid helium. The use of nitrogen for the initial cooling reduces the need for the more expensive helium.

The superconductor has been used successfully in a physies detector in Japan, so its performance has been proven. What has not been tried before is winding the conductor into coils with such a large diameter, and that difficult task still lies ahead. The reason for concern is that the superconductor is very flexible - a six-foot length of it, held up at the center, bends of its own weight.

To make a coil, the superconductor must be shoved into a holder, called a mandrel, where it will coil up in a way that resembles a coiled clay pot. Because of the superconductor's flexibility, however, buc- kling is a problem. To keep it from buckling, horizontal and vertical roller's will feed the superconductor into the mandrel, giving it the necessary support.

Perhaps the biggest challenge lies in reaching a uniform magnetic field of one part per million and then keeping it steady over the duration of the experiment. The field is affected by many factors. To ensure that the magnetic field is uniform, the coil positions will be surveyed repeatedly as the ring is assembled. After the ring is built, sensitive probes of the field will be used to look for variations. The magnet will have a special air gap between the poles and the rest of the magnet iron, which will allow for fine shimming of the field by inserting pieces of iron. The careful shimming process, including the use of special small coils attached to the pole faces, is expected to take a year and to improve the field quality by a factor of 100 . 


\section{Physics Department}

Researchers in the Physics Department study the fundamental constituents of matter and seek to understand the forces through which these constituents interact to form nucleons, nuclei, atoms and ordinary matter. Specialists in theoretical and experimental high energy physics, nuclear physics and solid state physics investigate a wide range of phenomena, probing the most submicroscopic building blocks of matter, quarks and leptons, and studying macroscopic effects such as the new high temperature superconductors.
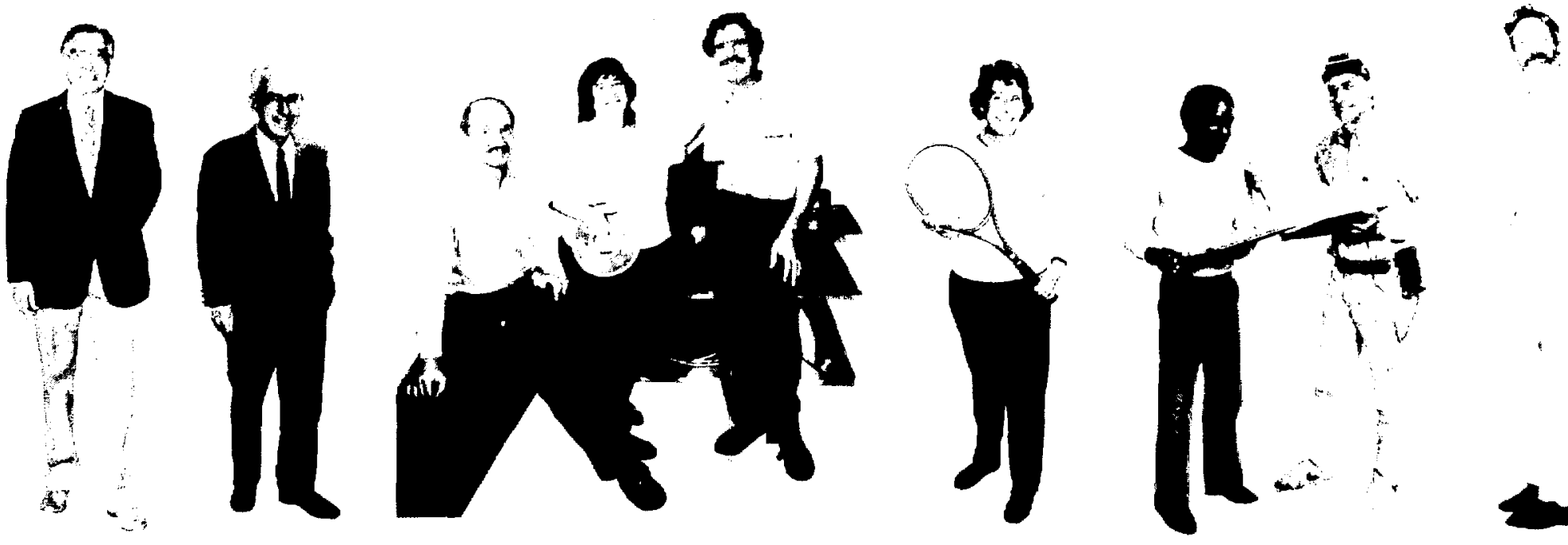


\section{The Puzzle of the Positrons}

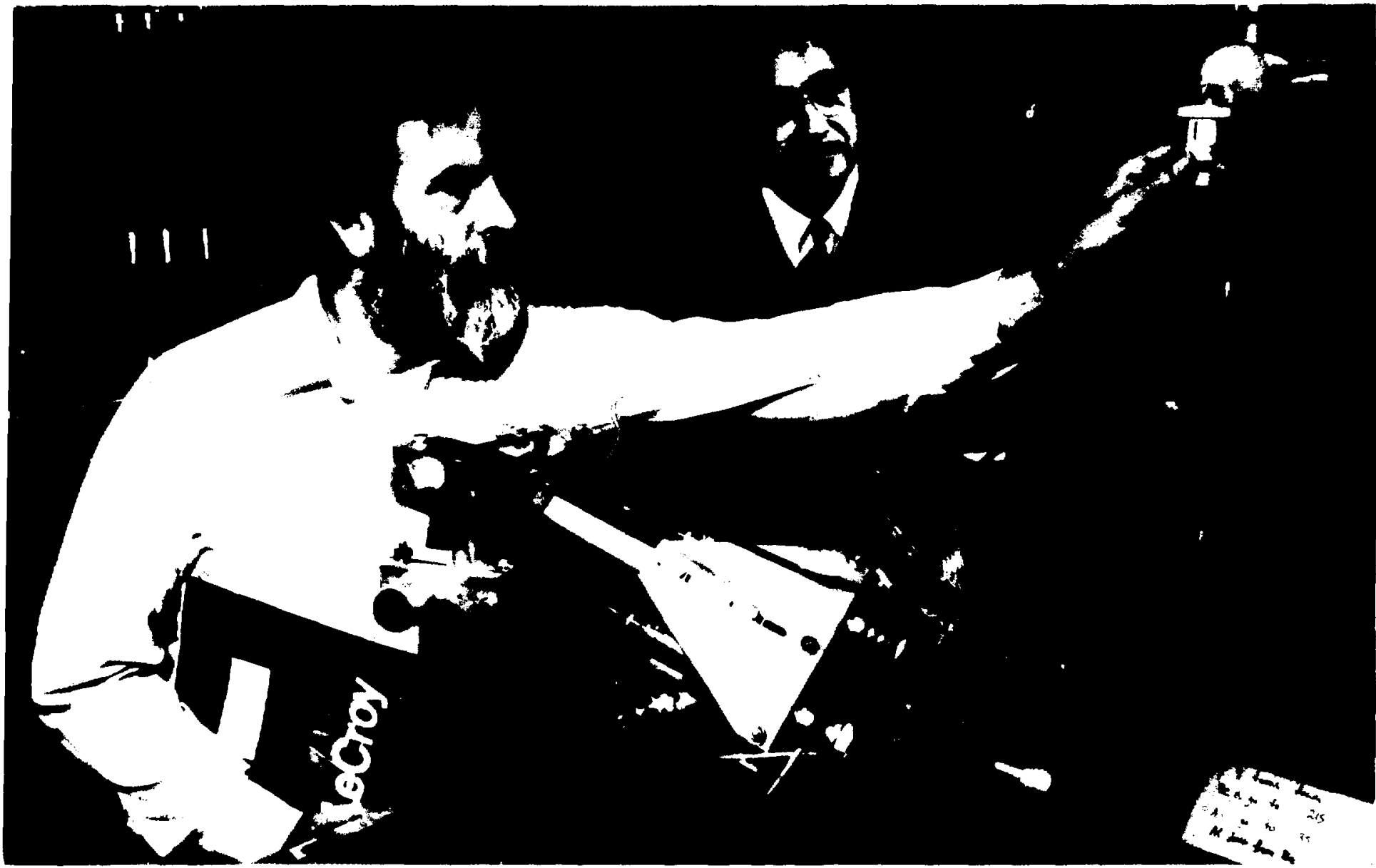

Brokharen s Kelein Lymn (left) and Jack Greembery. Vale University, are adjusting the detector system used in the Dymamitron in the searth for mon, neutral boson states.

$\mathrm{I}_{\mathrm{t}}$ Its existence was first suspected by researchers who noticed unusual events occurring in experiments on heary ion collisions. Physicists were intrigued - theorists tried to identify it. and experiment alists worked to capture it.

What was it?

In an effort to answer that question, scientists in Brookhaven's Physics Iepartment have teamed wijth other researchers from laale Iniversity (who first noticed the unusual events) and a group of scientists from the ('ity college of New York, in a new experiment. Their aim is to see if the source of the puzzle can be an unknown par ticle - and if so, to detect and identify it.

The existence of a new particle could explain the puzzling observations made by a Yale physicist and his team during experiments at the Gesellschaft für S'chwerionenforschung (GSI) scientific laboratory in Iarmstadt. West Germany. Originally, the cisi experiments had been part of another search - a search for spontancous positron emission, a fundamental process never before observed. Posit rons are posit ively charged particless. $c^{\circ}$, the antipart $i$ cle of the negatively charged elecetrons. a , which orbit the mucleses in an allom
A superheavy nucleus can have a vacancy in the lowest-lying electron orbits around it. Theory predicts that under certain conditions, if the nucleus is sufficiently heary and highly charged, this vacancy can be filled by a spont aneous sparking of the vacuum state, which is normally dormant. This leads to a new state of matter.

So experiments that smash heavy ion nuclei into each other were started in order to arhieve the superheary nuclei needed to init iate the process. I)at a were collected on the energy speetrum of positrons emitted during the collisions.

In this search for spont anteous sparking, another, inexplicalle. effect oceurred. Very narrow sharply delined posit ron peaks alwars appeared al about the same energies, distinet from the smooth 
background formed by the other positrons seen.

Possibly more interesting is that these positron peaks were then also observed in conjunction with similar electron peaks. Among the possible explanations, the best seemed to be that the conditions of the experiment favored the formation of an unknown, composite, neutral particle that then decayed back into an $\mathbf{e}^{+} \mathbf{e}^{-}$pair.

Physicists at Brookhaven and Yale decided to close in on the possible new particle from the opposite approach: they will start with the decayed components and hope to form the particle. A positron beam will be guided to bombard a target with electrons under conditions closely resembling the original experiment. The electrons will scatter the positrons normally, in what is called Bhabha scattering. If the new particle is formed, evidence of its presence should show clearly as a peak above the normal scattering background.

Setting up the experiment requires very precisely designed apparitus, because an extremely highly focused positron beam has to be used. Then the electron and positron from the scattering must be located very precisely in space, and their energies have to be exactly measured. For a maximum signal, the target electrons must be as nearly at rest as is possible. Suitable choices are beryllium or lithium, which have very low energy (and thus comparatively slow moving) electrons.

The necessary brightness and energy of the positron beam has been achieved by modifying Brookhaven's Dynamitron Accelerator facility. The final radius of the beam profile is only a single millimeter, and it diverges much less than one tenth of a degree, making it extremely intense. Also critical to the experiment's success is the stability of the beam's shape, position and intensity, so the beam is con- stantly monitored by another specially designed system.

When the beam strikes the target, scattered electrons and positrons are detected by wires in gas chambers located behind the target. The wires measure the particles' scattering angle, giving their position to an accuracy of better than one degree. Behind the chambers, eight scintillators detect the particles' energy. Thus, for each event, emission angle, energy and arrival time of both electrons and positrons are detected.

Three types of scattering should be seen in the detectors: the Mott scattering of positrons from nuclei, which can be distinguished by the positron energy and by not having electrons paired with the positrons; normal, Bhabha scattering of positrons by electrons; and a third type of scattering, which would be due to the positron and electron pairs first forming a new particle and then reappearing after its decay.

Data from the second and third types of scattering will look much alike, but the third type will be discernible by a greater yield, or resonance, at the appropriate energy level corresponding to the conditions of the original experiments in Darmstadt.

In case of a negative result, many of the proposed theories may be ruled out, and an upper limit to the strength of coupling between the electron and the hypothesized particle can be obtained.

If results are positive, a totally new particle of matter will have been discovered . . . no small matter!

\section{Filling the Holes in IIdh-T Theory}

$\mathrm{H}_{\mathrm{i}}$ - materials that lose all resistance to the flow of electricity at temperatures that are reasonably inexpensive to maintain - were first proved to exist in early 1986. For this discovery, two scientists in Switzerland won the 1987 Nobel Prize in Physics. Another Nobel may await the scientists who explain how this amazing phenomenon works.

At Brookhaven, using facilities for basic scientific inquiry such as the High Flux Beam Reactor (HFBR), the National Synchrotron Light Source (NSLS) and the Alternating Gradient Synchrotron (AGS), theorists and experimentalists discuss ideas, confirmations, then new questions. In these cooperative interactions, observations from experiments at one facility can throw immediate light on work being done at another - a process that already has considerably extended world knowledge of superconductivity at high temperature $\left(\mathrm{T}_{\mathrm{c}}\right)$.
Besides discovering the world's second superconductor to become effective at a temperature of over 90 kelvins (about $-300^{\circ} \mathrm{F}$ ) and thus indicating that the first discovery was not a solo event, Brookhaven scientists have been responsible for several experimental firsts aimed at unraveling the mystery of high $T_{c}$ superconductivity.

One such first, in an experiment using a neutron diffraction technique at the HFBR, proved that the new materials were antiferromagnetic. This means that the spins, or angular momenta, of neighboring atoms in the material are lined up in an alternating up-down-up-down array. The discovery also leads to the suggestion that there is a relation between magnetism and high $T_{c}$ superconductivity.

Another magnetic property of superconductors is the "Meissner effect," named for the scient ist who discovered it in 1933. Superconductors exclude any magnetic field with 


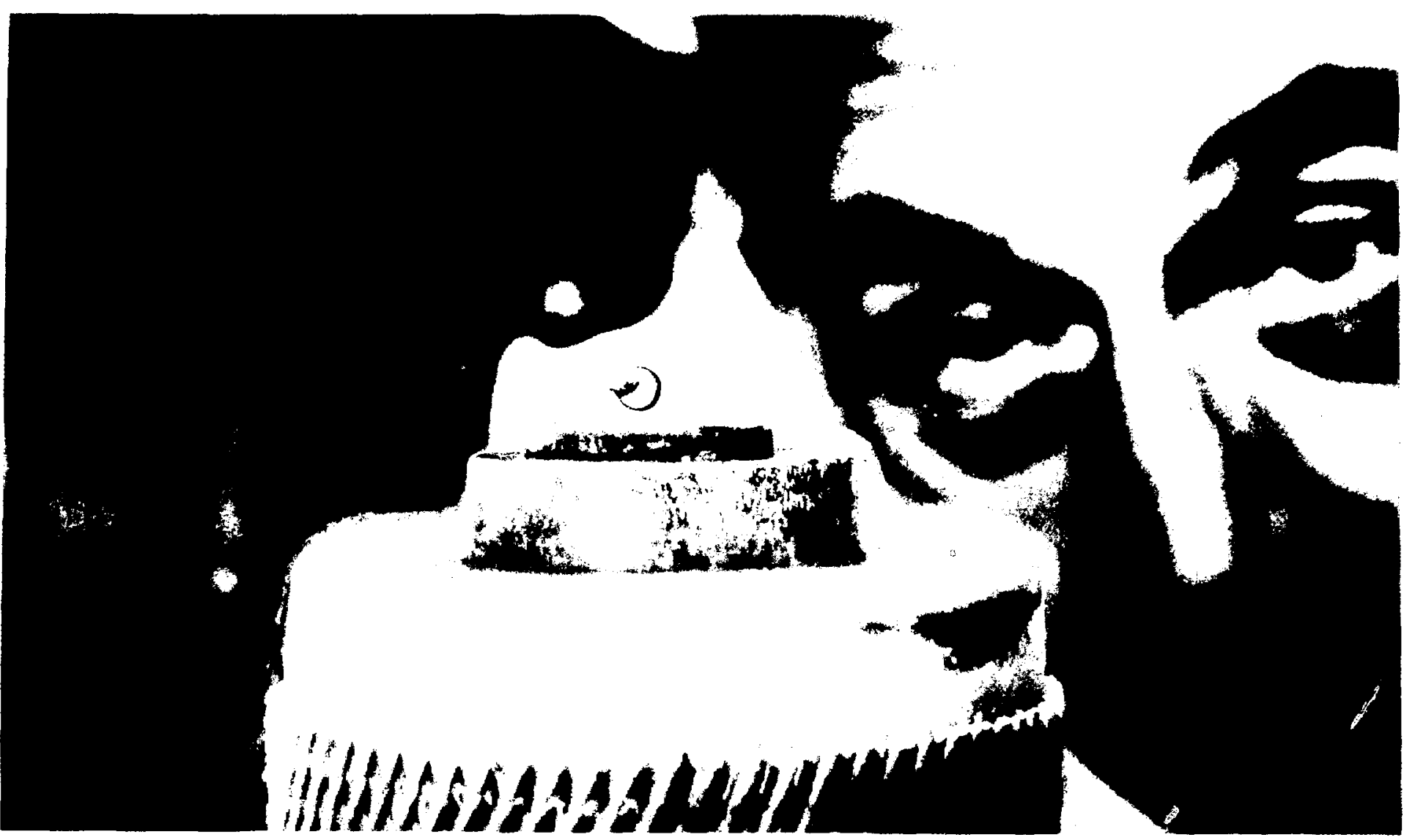

Brokhaten s edacational programs include a yearly series of seience lectures for selected Lomg Island high schersl stadents. Here, solid state physics theorist Virtor Emery explains the Messner effect to Angela Scraggs, from Bellport High Schosl. The . Meissner effect is demonstrated by the magnet flowting oner a hightemperature superconductor costed by liquid witrogen.

such force that a magnet is pushed away. This property led Brookhaven scientists to invent levitated trains. an idea now ready for commercial use in Japan.

The Meissner effect is often used as a test to determine whether a material is superconducting. Not all the magnetic field is expelled, however. Superconductors vary in how deeply they can be penetrated by a magnetic field.

A series of experiments to measure the penctration depth of different new superconductors was undertaken at the AGs, using a technique called muon spin relaxation. Researchers learned that the penetration depth depends on temperature and varies with the number of particles (the supereurrent charge carriers) per unit area of the sample, divided by theil mass.
This information is valuable in underst anding the nature and mechanism of the high $T_{c}$ superconductors, which differ in many ways from low $T_{c}$ superconductors. For example, in low $T_{c}$ superconductors, phonons, the smallest unit of vibrational energy of the material, are thought to interact with electrons, which surround the nucleus of each atom. The phonons cause the electrons to pair as distant partners. Then all these weakly bound pairs move together in a supercurrent.

Evidence from experiments at the HFBR suggested, however, that phonons did not mediate bet ween electron pairs in the high $\mathrm{T}_{\mathrm{C}}$. compounds. A different mechanism must be at work.

Another Brookharen team of researchers, using the $x$-rays at the isLs, found yet more new information. The researchers were invest $i-$ gating the electronic structure of high $\mathrm{T}_{\mathrm{c}}$ superconductors by $\mathrm{x}$-ray absorption experiments. Electrons orbit atoms in levels of different energies. The core levels, near the nucleus, are usually filled, but the outer layers are not. So an inner electron can be excited into moving to another level. In a solid, the outer layers of different atoms overlap, changing the outer energy levels and also changing the spatial dist ribution of the outer electrons, known as valence electrons.

One way to study the electronic structure of a solid is to measure the energy needed to excite an eloctron from a filled core level to the lowest outer energy level that is unfilled. The energies neoded generally lio in the $\mathrm{x}$-ray energy range. and the cerhnique used is $x$-ray alsorption spect roseops, performed at the NSLS 
The Brookhaven physicists studied the electronic structure of the compound lanthanum copper oxide as it was changed to a high $T_{c}$ superconductor by the partial replacement of lanthanum by barium or strontium.

They found that as the compound changed to a superconductor, the copper atoms remained unchanged, but that holes (that is, missing electrons) were created on the oxygen atoms.

Holes can act as particles in their own right. Like electrons, they have spin and they can pair. When the temperature drops to a certain level, holes on the oxygen atoms in the copper-oxygen planes of the superconductor can pair with neighboring oxygen holes having opposite spin. These pairs of holes then make up the supercurrent, which travels without resistance through the superconductor.

More information about the holes came from the penetration depth experiments at the AGS. These showed our researchers that the pairs used by the high $T_{c}$ superconductors to carry charge had no rotational motion.
These examples of the knowledge gained by different techniques used at Brookhaven, combined with other information discovered both here and elsewhere, show the considerable advances made in our understanding of the new superconductors. Much still remains to be learned. While research is greatly stimulated by the tremendous advantages promised by practical applications of these compounds, another, equally powerful motive for many scientists lies in uncovering new information in the field of solid state physics.

\section{Testing the Standard Model}

Once any theory becomes conventionally accepted, it's human nature to want to test it anew. Is the theory really as firmly established as it appears?

The standard model - the theory of matter generally favored by most physicists - is no exception. Scientists are continually doing experiments to check its accuracy. If their results match the predictions, the standard model is vindicated. If there is evidence of a contradiction, exciting new physics may follow.

One way to test the model is to probe for an unknown decay mode while examining particles as they decay into other particles. A suitable candidate for study is the kaon: Because it is a relatively long-lived particle, there is time to study its decays in very weak interactions. Brookhaven's Alternating Gradient Synchrotron (AGS) is the world's most intense source of kaons, making the facility a favored location for doing rare kaon decay experiments (such as the one on page 20.)

Our newest rare kaon decay experiment is E787, where the search is on for a positive kaon $\left(\mathrm{K}^{+}\right)$ that decays into a positive pion $\left(\pi^{+}\right)$, a neutrino $(\nu)$ and an antineutrino $(\bar{\nu})$.

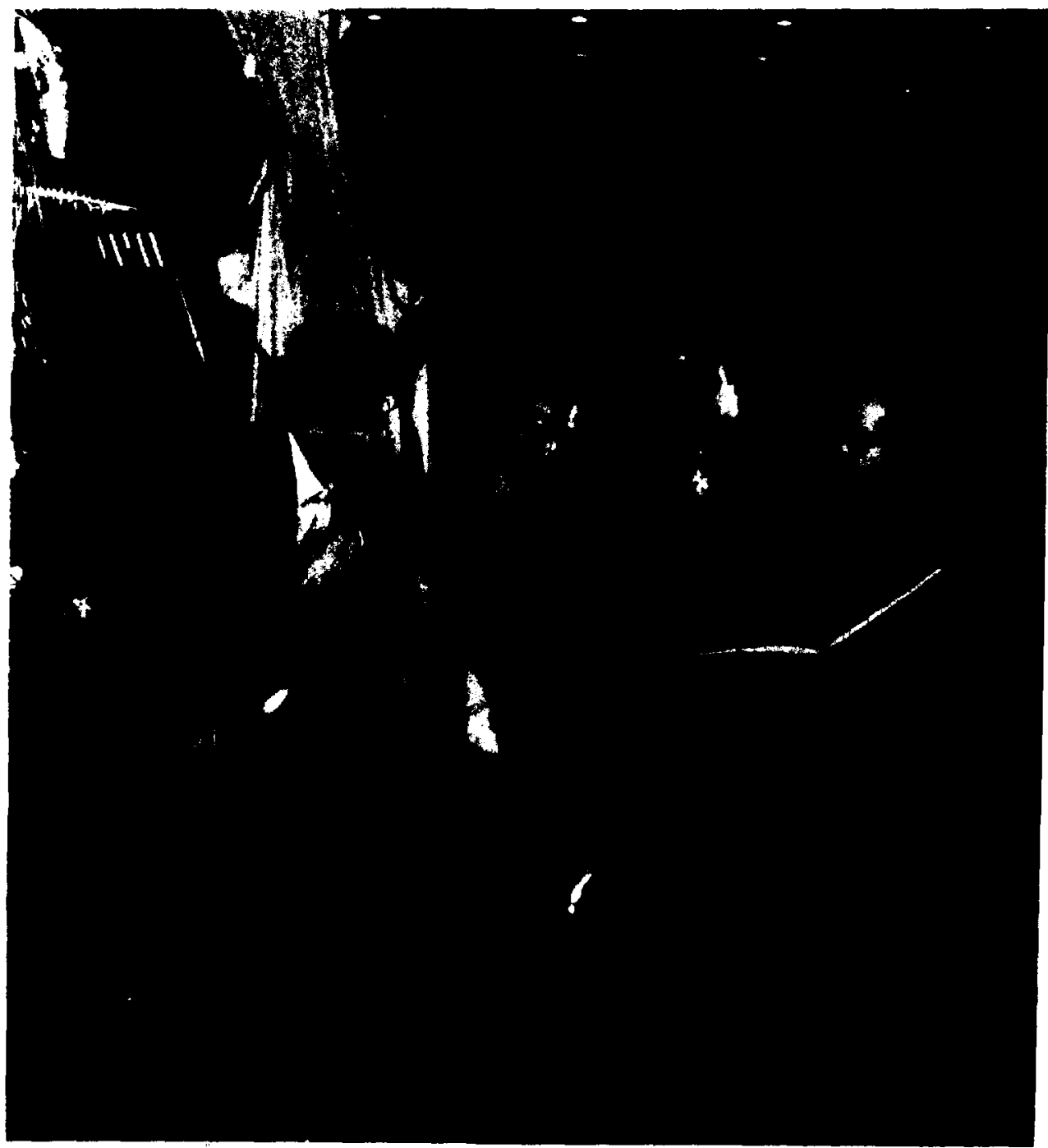

Checking on signals from the detector in front of the experiment are (from left) Ted Kycia, Kelvin Li, Maged Atiya and Laurence Littenberg. 
To track a decay, physicists set up apparatus designed to identify the particles that emerge when a kaon hits a target. Results from E787 are difficult to analyze, however, because neutrinos and antineutrinos are almost impossible to trace.

Neutrinos and antineutrinos are minute particles reacting so infrequently with other matter that they can pass through the entire earth without being detected. For example, billions of billions of neutrinos from the supernova SN1987A explosion seen in February 1987 streamed through the earth. Yet only 19 of them were observed, eight in the United States and 11 in Japan.

The E787 experimenters, therefore, cannot expect to track any neutrinos that might result from their kaon decay. Only the energy of the positive pion can be traced, giving just part of the total sum of the energy that emerges from the decay.

Thus, identifying the pion is vital. E787's detector is set up to make sure that if a single pion appears, it will be recognized. If a pion is emitted along with other particles, such as a gamma, this signals a different decay.

The process begins with a beam of hydrogen ions being accelerated to 30 billion electron volts and smashed into a target of protons and neutrons. The kaons that emerge from the particles formed by the collision are guided into the E787 apparatus. E787 differs from the other rare kaon decay experiments under way at the AGS in that the kaons, rather than decaying in flight, are slowed down by a material called a degrader and then stop in the target. After that, they take about 12 nanoseconds to decay by emitting low energy particles.
Each particle travels through the heart of the detector, which is located in a large solenoid magnet that bends the particle's path so that its momentum can be measured. The particle then traverses another section of the detector that contains a stack of scintillation counters, which measure energy and range. There, it loses energy until it stops. The relationship among measured momentum, range and energy is characteristic of a particular particle.

E787 can be expected to produce perhaps two events over the course of each experimental run. If there is a pion from the decay $\mathrm{K}^{+} \rightarrow \pi^{+} \nu \bar{\nu}$, it will subsequently decay into a muon and a neutrino. Then the muon will decay into an electron, an electron neutrino and an antineutrino.

Part of E787's apparatus helps to detect the muon and electron, to confirm the pion's presence yet further. The equipment is also designed to help rule out any other decay modes, to an accuracy of less than one mistake in ten billion events.

What happens if $\mathrm{K}^{+} \rightarrow \pi^{+} \nu \bar{\nu}$ is observed? In the standard model, this decay is known as "highly suppressed" - very unlikely to happen, but not a violation of the model if it does occur. Should our results indicate that the likelihood of seeing the decay is about the same as that predicted by the standard model, the model will be confirmed. Should the number of decays we observe be higher, showing a higher probability, this could indicate that the standard model needs to be changed, perhaps to include a new generation of unknown particles.

A higher count might have another explanation, however. Instead of the $\mathrm{K}^{+} \rightarrow \pi^{+} \nu \bar{\nu}$ decay, some of the decays might turn out to be $\mathrm{K}^{+} \rightarrow \pi^{+} \mathrm{X}^{0} \overline{\mathrm{X}}^{0}$. In this case, $\mathrm{X}^{0}$ could be a new particle, such as the "sneutrino," the "photino," the "goldstino" or the "majoran." These are predicted by current theories competing with the standard model.

Another explanation might lie in the decay $\mathrm{K}^{+} \rightarrow \pi^{+} \mathrm{X}^{0}$, where $\mathrm{X}^{0}$ is a yet-undiscovered neutral particle, light and weakly interacting, such as an axion, a familon or a Higgs scalar.

Should either $\mathrm{K}^{+} \rightarrow \pi^{+} \mathrm{X}^{0} \overline{\mathrm{X}}^{0}$ or $\mathrm{K}^{+} \rightarrow \pi^{+} \mathrm{X}^{0}$ appear, the E787 detector is designed to pursue the investigation further. Whatever the answer, the final result will be interesting and revealing physics.

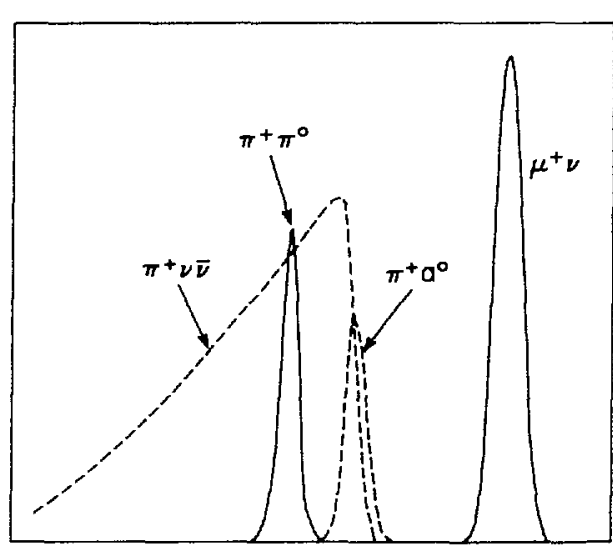

Relevant data collected for the $K^{+} \rightarrow \pi^{+} \nu \bar{\nu}$ decay are represented by the dotted line rising into a rounded curve. This differs from most kaon experiments, in which the signal appears as a sharp, easyto-recognize peak, such as $\pi^{+} \pi^{0}$ and $\mu^{+} \nu$ in the figure. Aside from the possibility that $K^{+} \rightarrow \pi^{+} \mathrm{X}^{0}$, any peaks that do appear are useful to E787 experimenters as a signal of where not to look. Should an axion appear, the data might follow the curve labeled $\pi^{+} \alpha^{0}$. 


\section{Department of Nuclear Energy}

The Department of Nuclear Energy is involved in analyzing the safety of nuclear power reactors, studying advanced reactor systems, developing improved methods of safeguarding nuclear materials, investigating radiological hazards, and compiling and evaluating nuclear data required by scientific users throughout the world. In recent years, the department has expanded its activities to include new programs under the federal government's Strategic Defense Initiative.
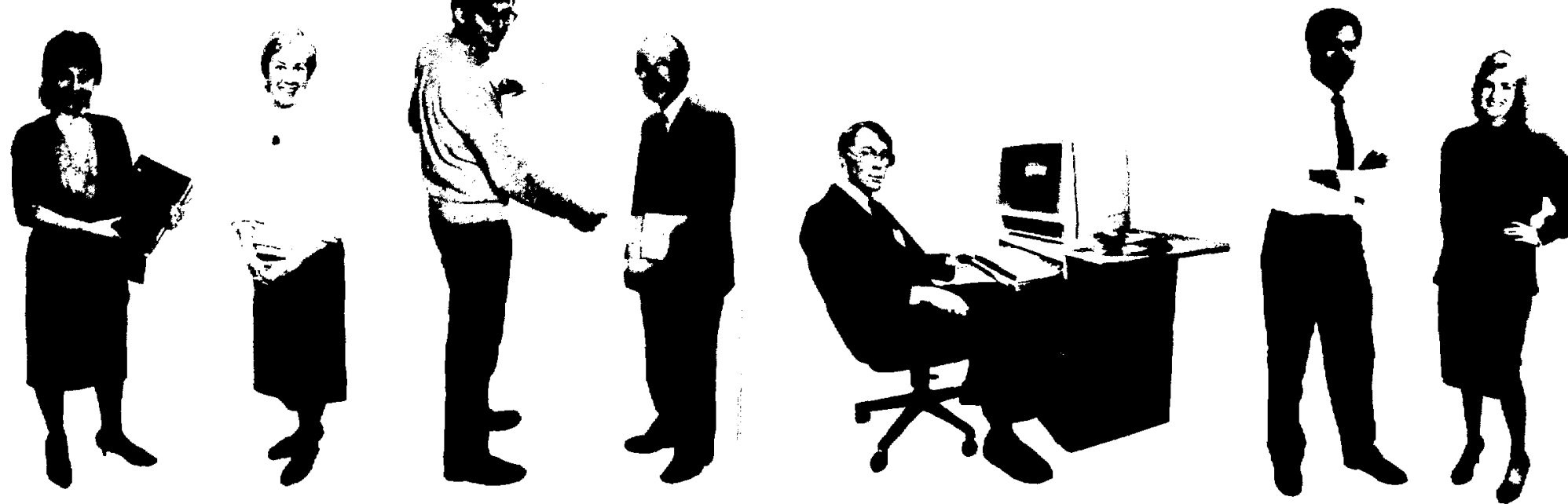


\section{Answers in Short Time}

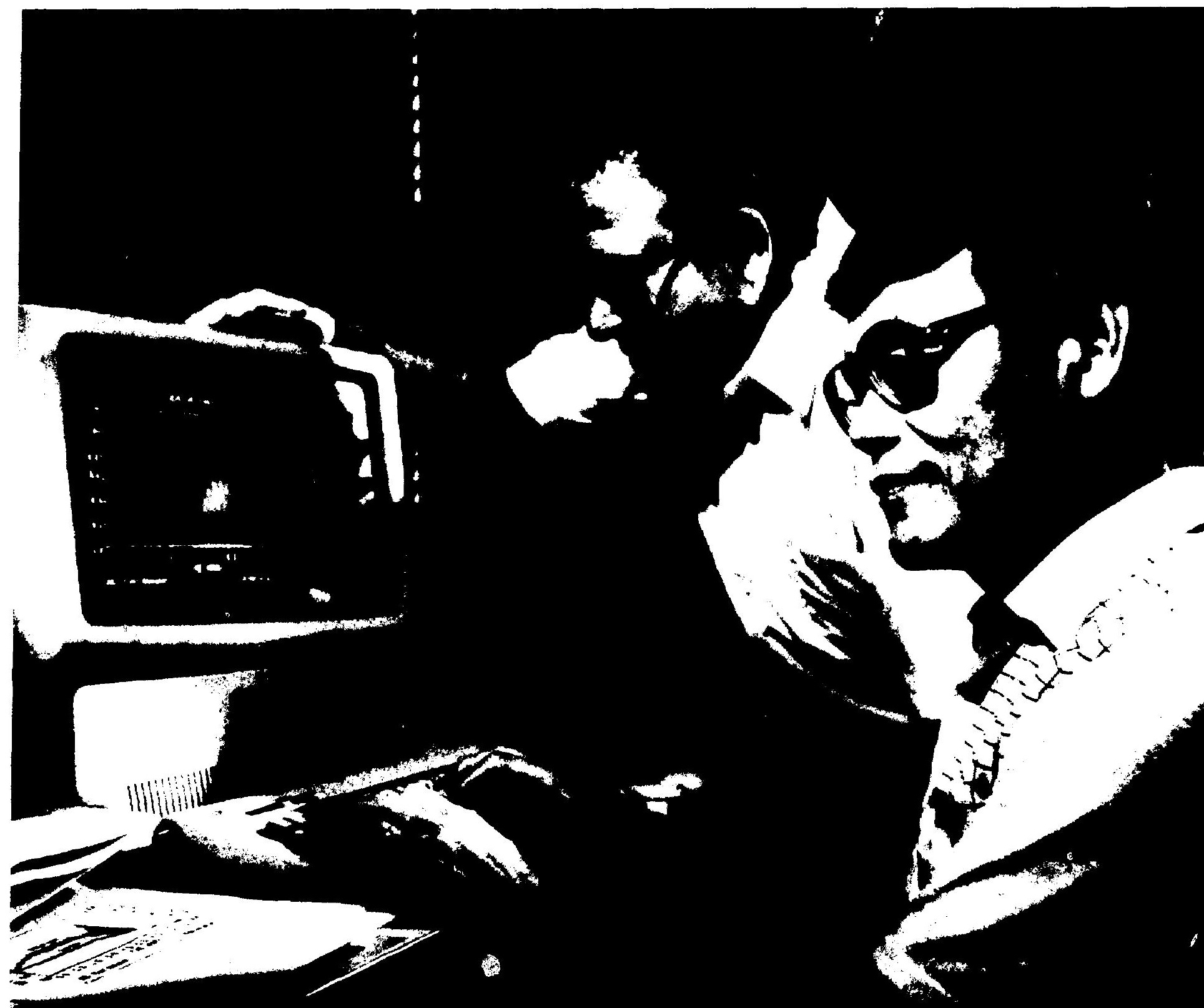

Hsiang-Shou Cheng (foreground) and Wolfgang Wulff examine power oscillations at the LaSalle 2 nuclear power plant, as simulated by BNL's Engineering Plant Analyzer. 
It was 1938 when the first paper was published on hydraulic instability. The phenomenon can occur in nuclear reactors, specifically boiling water reactors ( $B W R$ ), but it has been so well studied that standard reactor design and operation prevent it from happening.

On March 9, 1988, however, the unexpected occurred at the LaSalle 2 nuclear power plant in Illinois. On that day, the power began to oscillate, and these oscillations grew quickly as the heat generated by the reactor core no longer matched the cooling water flow for stable cooling. The reactor operators tried to restore stable operation, but failed. Five minutes into the event, as the operator's were preparing to scram (shut down) the reactor manually. it scrammed automatically.

Because of our ability to respond quickly, Brookhaven was asked by the Nuclear Regulatory Commission (NRC) to find out what had happened and why. We tackled the assignment with our Engineering Plant Analyzer, a fast and reliable computer system, and in a week had an assessment: The power oscillations were caused by hydraulic instability, which can occur under the unusual condition of high power combined with low coolant flow.

There were no consequences to the public from the LaSalle event since the safety systems responded as they should, but the incident raised questions about hydraulic instability.

LaSalle is a BWR, and power oscillations are known to occur in this type of reactor. In order to prevent hydraulic instability, operators are trained to respond to power oscillations with a routine recovery procedure that does not require shutting down the reactor. Once down, a reactor takes 24 hours to restart, and the loss of electricity for one day costs the utility about one million dollars.

The LaSalle incident was triggered during a maintenance procedure,

when a technician closed the wrong valve while testing a pressure switch. This caused both recirculation pumps to trip off, greatly reducing the flow rate in the core. That much we know for sure. The following scenario is based on our simulation of the event with the plant analyzer.

Within seconds, the reactor was in a high power/low flow condition. Normally, natural circulation is enough to keep the reactor stable in this condition until the pumps can be started again. This time, it wasn't. The inadvertent valve closure shut off a feed-water preheater, and cold

\section{On to PWRs}

The United States has 107 nuclear power plants in commercial operation. This includes 35 boiling water reactors (BWR) and 71 pressurized water reactors (PWR).

Brookhaven's Engineering Plant Analyzer was originally designed to simulate the plant performance of BWRs only. In the fall of 1987 , we began the greater task of developing a plant analyzer for PURs - a job that will take about three years to complete. This project is being supported by the Nuclear Regulatory Commission; the Empire State Electric Energy Research Corporation; both the government and the nuclear industry of Taiwan; and Applied Dynamics International, the manufacturer of a special purpose minicomputer, the newest generation of the one we use in our BWR plant analyzer.

In a boiling water reactor, steam is generated in the reactor vessel itself. The steam then goes into a turbine, which drives an electrical generator. In a pressurized water reactor, liquid in the reactor vessel is heated (under pressure to prevent steam forma- feed water entered the core. The cold water intensified the power oscillations, which quickly grew until they were swinging from 30 to 120 percent of normal. When the power peak reached the set point of the automatic reactor protection system, the reactor automatically scrammed.

Using the plant analyzer to simulate and speculate on different ways that the operators could have intervened, we learned that restarting the recirculation pumps and rapidly restoring full flow would have been effective in damping the power oscillations, but it would have

tion), and the heat is transferred in a steam generator outside the vessel to produce steam, which rotates a turbine that drives an electrical generator.

Although both types of reactor produce the same amount of power, up to about 1,000 megawatts of electricity, a PWR is a much bigger system because of its steam generators and other additional equipment. Hence, the job of simulating a PWR is much greater.

Up to the task is the new minicomputer for this project. A parallel processor similar to the computer used for the BWR plant analyzer, the new machine is larger, faster and able to deal with decimal numbers. This last feature, in particular, gives the system much greater precision in simulating an operating plant.

As we have done with the BWR plant analyzer, we will be using the drift flux model to simulate PWRs. We favor this model because it reflects the experimental data base better than any other model. And since the model relates directly to experiments, speculation and hypothesis are eliminated, giving us great er confidence in the results. 
caused the reactor power to increase rapidly, tripping the reactor even sooner. Instead, the best course of action for that event would have been to restore the flow slowly to about 50 percent of normal, which would have stablized the reactor and prevented the automatic scram. An alternative would have been to insert the control rods to reduce power, although that would not have been as effective at that particular point in the reactor's operating cycle.

What if the reactor had not shut down automatically" We simulated the oscillation with a disabled scram system for 20 minutes and found that the power would have oscillated between 20 and 500 percent of normal full power.

Even though there were no con- sequences of the LaSalle incident, the potential effects of power oscillations are not well understood under conditions of multiple failures, particularly considering such factors as improper operator action and equipment failure. After having carried out the LaSalle analysis, we were next asked by the NRC to take on the long-term project of evaluating the generic implicat ions of hydraulic instability in BWRs.

Brookhaven's assessment of LaSalle is the latest demonstration of the versatility and quick-response capability of our plant analyzer. A critical key to its success is the use of a special-purpose minicomputer, which is superior to a large mainframe computer because it carries out the same calculations a hundred times faster. In addition, while there are various flow models to simulate the reactor eolant for the day-to-day operation of a plant. as well as during various hypothet $i-$ cal transients (events that cause the plant to change from a st eady-st ate condition ), we favor the drift flux model over more complex models because it has proven to be closest to the available experiment al dat a base.

Yet another advantage of the system is that it can be called up by telephone and used remotely. The NRC routinely accesses the plant analyzer from its headquarters in Washington, D.C., and our st aff has used it in demonstrations from as far away as Spain, Italy, Taiwan and Korea.

\section{A Technical Knockout}

Involved in the seismic stress test of part of a nuclear pouer reactor were (seated, from left) Charles Hofmayer and Walter Kato, and (standing, from left) Semyon Shteyngart, John Curreri, Young Park and Y. K. Wang. On the table is a piece of the 14-inchdiameter coolant pipe that was part of the model subjected to a simulated earthquake bigger than any ever experienced.

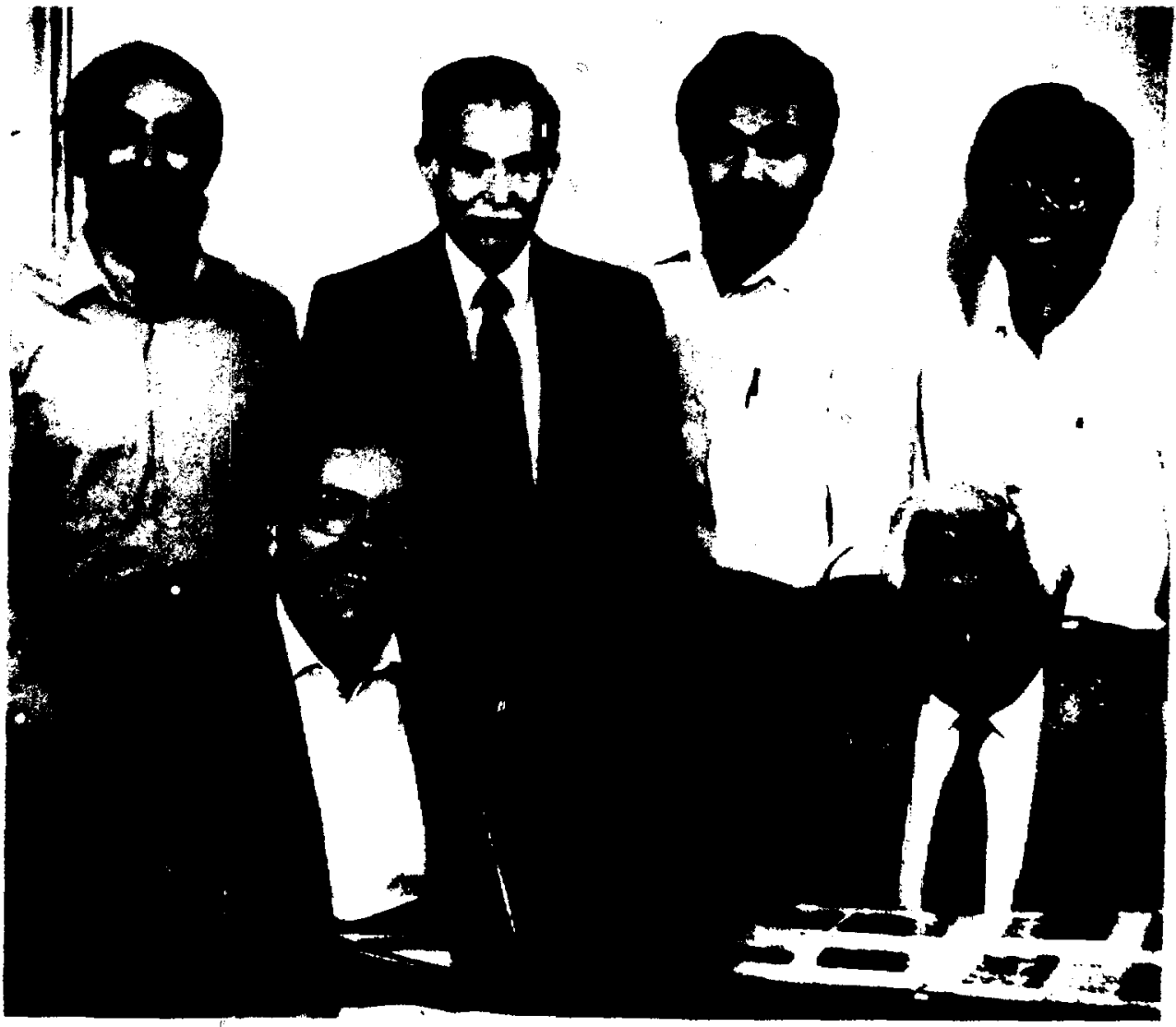


In April 1988, an earthquake bigger than any ever experienced - or ever expected to occur - was simulated in order to test a one-thirdscale model of a nuclear reactor's primary coolant system. What happened? A technical knockout, as predicted. A large stainless steel pipe was permanently deformed.

The test took place at the Tadotsu Engineering Laboratory in Japan, using the largest vibration table in the world, and was the first attempt ever to subject such a large structural system to so high a seismic stress level. It was the culmination of three years of preparation under an international agreement between $\mathrm{BNL}$, as the representative for the U.S. Nuclear Regulatory Commission (NRC), and the Nuclear Power Engineering Test Center in Japan, the representative for the Japanese Ministry for International Trade and Industry (MITl). Also participating were Japanese utilities and the Electric Power Research Institute in the U.S.

This agreement is the first between the NRC and MITI to call for scientific cooperation on structural and mechanical engineering for nuclear power plants. BNL was involved because of our well-known expertise in structural and seismic analysis, and we were responsible for guiding the program.

Initially, we did a feasibility study of the proposed test. During this phase, we chose the structural system to be tested, decided on the seismic excitation and performed calculations to predic the structural response. A sophisticated computer code was used to study the dynamic motion and the material behavior of the test model. All this was done to insure that the simulated earthquake was powerful enough to bring the model to a near-failure condition.

In Japan, which relies heavily on nuclear power, all plants must be designed to withstand major earthquakes without even minor damage.

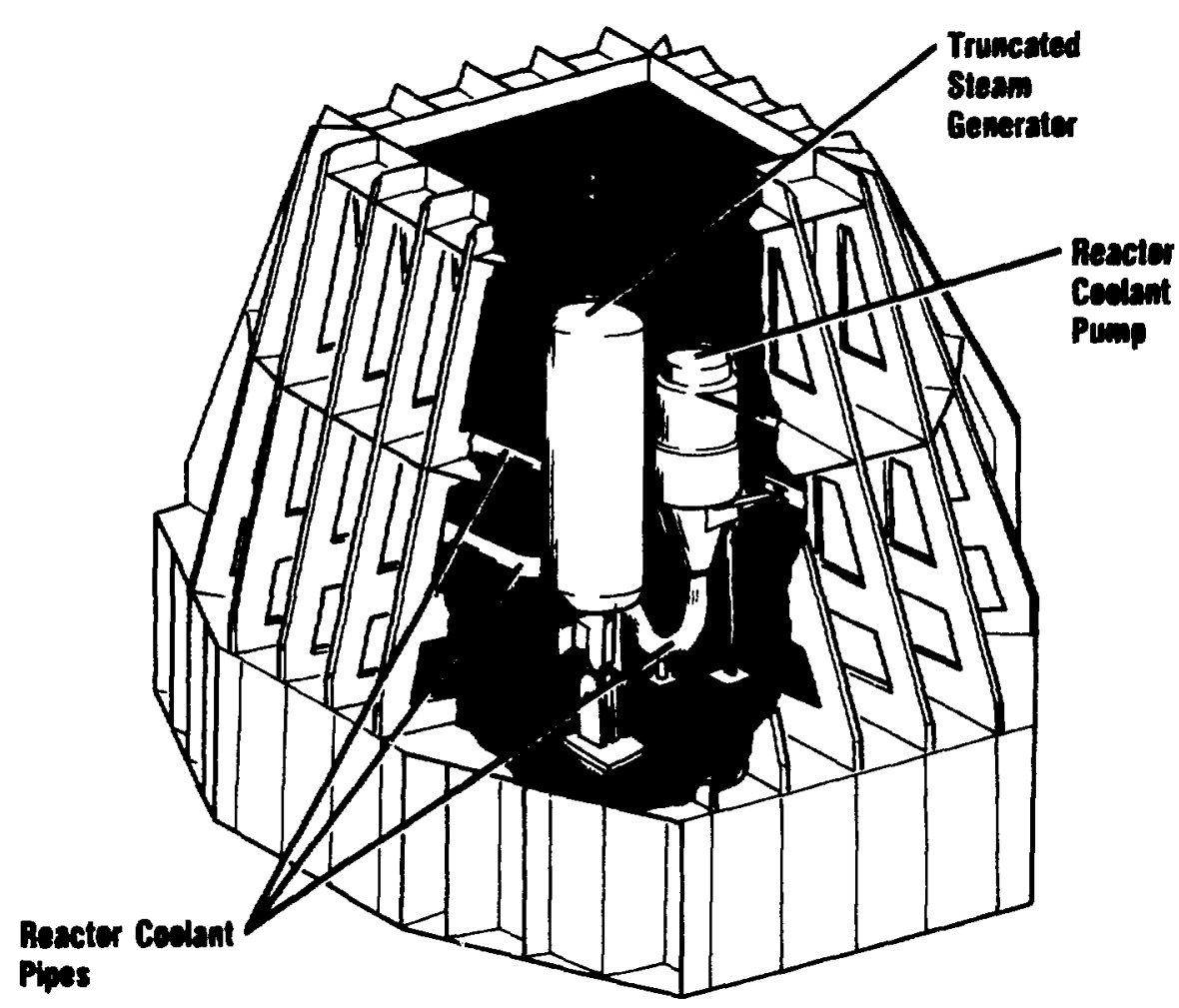

This cutaway drawing shows the reactor coolant system model, housed within the frame that supported it for the earthquake test. Modified for the test, the coolant system consisted of a reactor coolant pump, a truncated steam generator, and reactor coolant pipes that uould ordinarily connect the steam generator and coolant pump to a reactor vessel.

The Japanese islands are located in an area in which the ocean floor is moving under the Asian continental land mass, and this movement produces great geological stress that can result in large earthquakes.

As the federal agency that regulates commercial power plants in the U.S., the NRC carries out research to learn about the capability of nuclear plants to withstand earthquakes. Since experiments of nuclear power plants under earthquake motion are very expensive, current earthquake-proof design of large structures is based primarily on computer analysis. Computer simulations are used to predict the behavior of components during earthquakes, and the results are used to specify materials, their dimensions and the necessary sup- ports for various components.

Individual components are tested, but because components interact when they are assembled into structures within a plant, actual test data on how a large structure behaves during an earthquake is largely missing. For the NRC, then, the simulated earthquake in April marked the first large-scale test of a nuclear reactor primary coolant system.

The system consists of a steam generator, pump and piping, all connected in a loop to deliver cooling water to the core of a nuclear reactor. For the test, a one-thirdscale model of a typical coolant loop for a pressurized water reactor was manufactured at a Japanese industrial facility where actual reactor parts are built. 
To stress the structure to a high level - to try to make it fail - the upper and middle lateral supports of the steam generator were removed. As a precaution against damage to the vibration table, the top part of the steam generator was cut off.

Measuring 15 by 15 meters, roughly half the size of a basketball court, the Tadotsu vibration table can hold a maximum load of two million pounds. All together, the coolant loop model, plus a frame built to support it during the test, weighed about one million pounds. The table subjected this load to a maximum acceleration of almost three times gravity - more than 15 times the maximum value of what most U.S. nuclear power plants are designed to withstand. In addition, the "earthquake" was applied in the worst possible dynamic way.

The test was done in a series of stages, each one increasing the vibration until the limit of the table was reached. A crack appeared on one 14-inch diameter pipe only after the second maximum excitation run. Two additional excitations were carried out, with the crack growing each time. When the test was over, the crack was at a depth of approximately 30 millimeters $(\mathrm{mm})$, in an area where the thickness of the pipe is about $32 \mathrm{~mm}$.

Hence, the structure did fail, but not catastrophically. Also, it failed in a way that had been predicted. In engineering parlance, the pipe went plastic - it permanently deformed because of the extraordinary stress put on it.

The test demonstrated that under current NRC regulations, the piping in a nuclear power plant coolant loop is designed with a very large seismic safety margin. What was also gained from doing the test was extensive data, which are needed to validate the computer codes used to assess the safety of nuclear power plants under severe earthquake loading. Also, since the piping was pressurized and the maximum excitation repeated several times, we will be able to study the effects of crack propagation, fatigue and ratchetting (a cumulative process of cyclic strain).

In this country, the philosophy is to continually upgrade knowledge to maintain realistic and safe regulations. The successful seismic testing program done in cooperation with Japan is yet another way to maintain that high standard of safety.

\section{Accelerator Technolody for Defense}

$T_{\text {he }}$ (SDI) was proposed by the federal government in 1983 . Since then, both industry and national laboratories have been working towards the development of a combined land- and space-based ballistic missile defense system.

At Brookhaven, we are addressing basic physics issues to determine the feasibility and potential of one of the elements of the concept, namely, neutral particle beams, which might be used to identify and destroy hostile missile booster and reentry vehicles before they reach U.S. territory.

\section{Two New Facilities}

In support of this effort, in July 1985 we began construction of the
Radiation Effects Facility (REF) and nearly a year later, broke ground for the Neutral Beam Test Facility (NBTF).

Studies at the REF, which began operating in October 1986, center on radiation effects on various materials and components, mainly electronic. For example, a single solar proton going through a computer chip operating in space can cause computer error even though the chip itself is not damaged. Survivability of electronic components in space is important, and a substantial effort is directed at developing radiation-resistant devices.

The NBTF was commissioned in May 1988. Although research there is not yet fully under way, experiments at the NBTF will focus on the development and testing of neutralizers and neutral-beam-sensing and control devices.

\section{Linked to the Linac}

Both the REF and the NBTF are linked to the Laboratory's existing 200-million-electron-volt linear accelerator (linac). The linac is used primarily as an injector for the Alternating Gradient Synchrotron (AGS), Brookhaven's premier particle accelerator for physics experiments.

Since the linac produces more protons than the AGS requires, the excess particles have long been diverted and used to produce radionuclides for use in medicine and industry. Now, surplus protons from the linac are also helping 


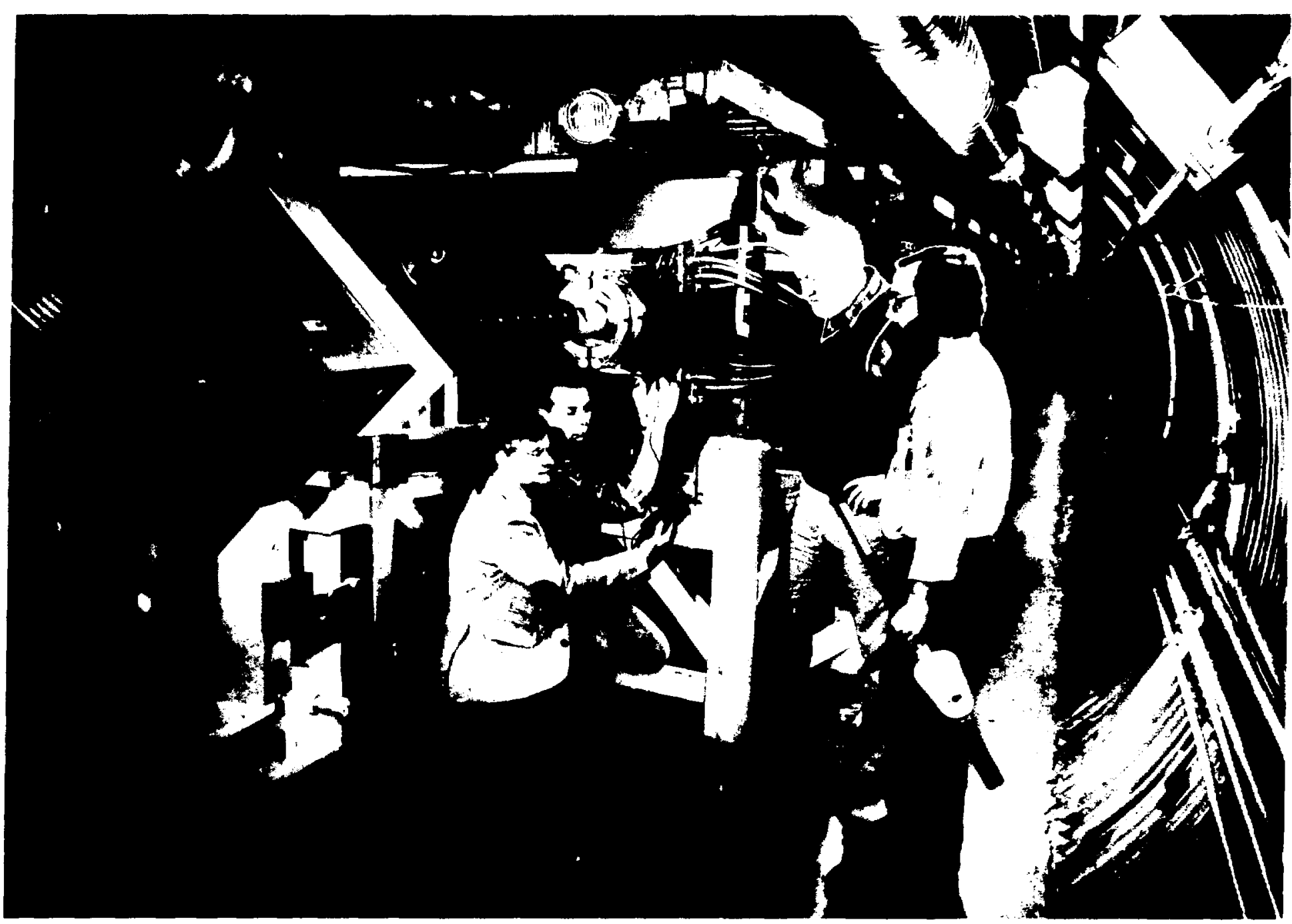

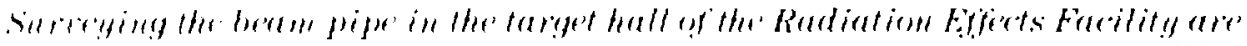

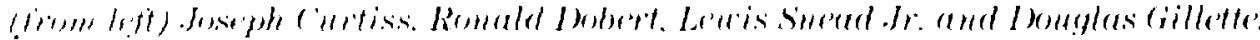

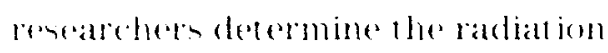

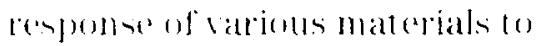

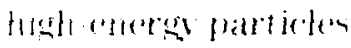

\section{REF Research}

\section{'The liEt' is atalathleto}

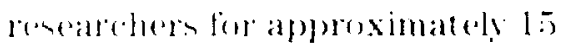

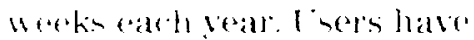
imeluched national laboratories. sueh

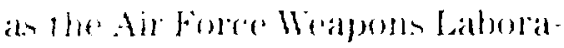

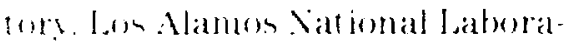

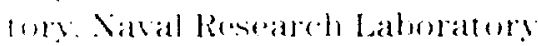

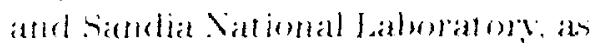

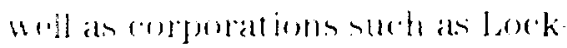
lienerland Trits.

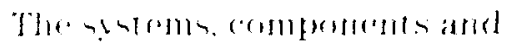
materials that thene merablizat ioms

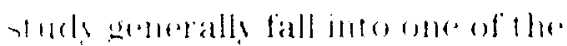
folloming rexperimental atroks

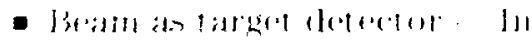

this 19 ) of rescareh. experimenters want lo determine whether a meth

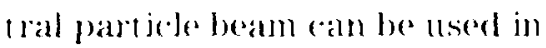
space 10 diseriminate betweren a

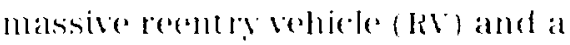

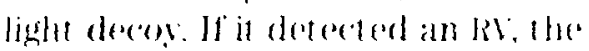
heatu might then herome a defent sive weapon. With the goal of hitting and destroping the missile.

- Interactions with propellants Researehers wonder What wonld happen if the beam anme in comtace with the slow hurning propellame of missiles and Ris. Rather than burn ing a propellant slowls imward, the

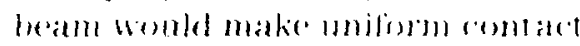
with it. produreing greatl hralt alld perthates callesing lhe aloms in the

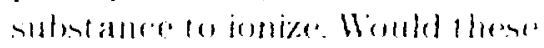

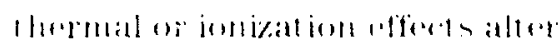

the behavion of the propellant, pere hape causing it to decompenesor berome inarelive". ( omld heat ind of lhe propellant result in an emhancement of the butn rates:

could this catlse the mission to fail"?

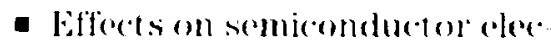
tronics - Tho spate program has

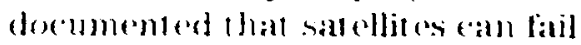
when nat ural spater ratiat ion feos mic lays and solar wind) interates with elecetromics. Researeleres are

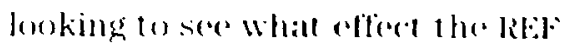

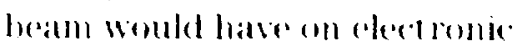
(o)mproments in missiles and Ris in j)alce.

Whemerer possthle. thin informa fioll is entrelated with rathation

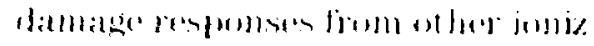

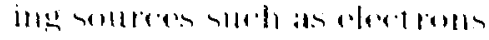


gamma rays and neutrons. In this way, the REF will help provide a broader base of understanding of transient radiation effects in electronics.

- Responses of other components - Among the long list of various other components being tested at the REF are those such as gyros and accelerometers, which keep missiles and RVs on their course.

\section{Looking Ahead}

Although accelerator technology is of obvious interest to the military, what is particularly important to
Brookhaven in the long run are the new areas of basic research that these facilities open up.

Apart from the federal government's SDI effort, Brookhaven expects steady growth in the years ahead in basic physics research at both the REF and NBTF. In particular, we see the potential for doing world-class research in neutron tine-of-flight physics, for studies of nuclear interactions as they pertain to QED, or quantum electrodynamics, the theory of electricity and magnetism.

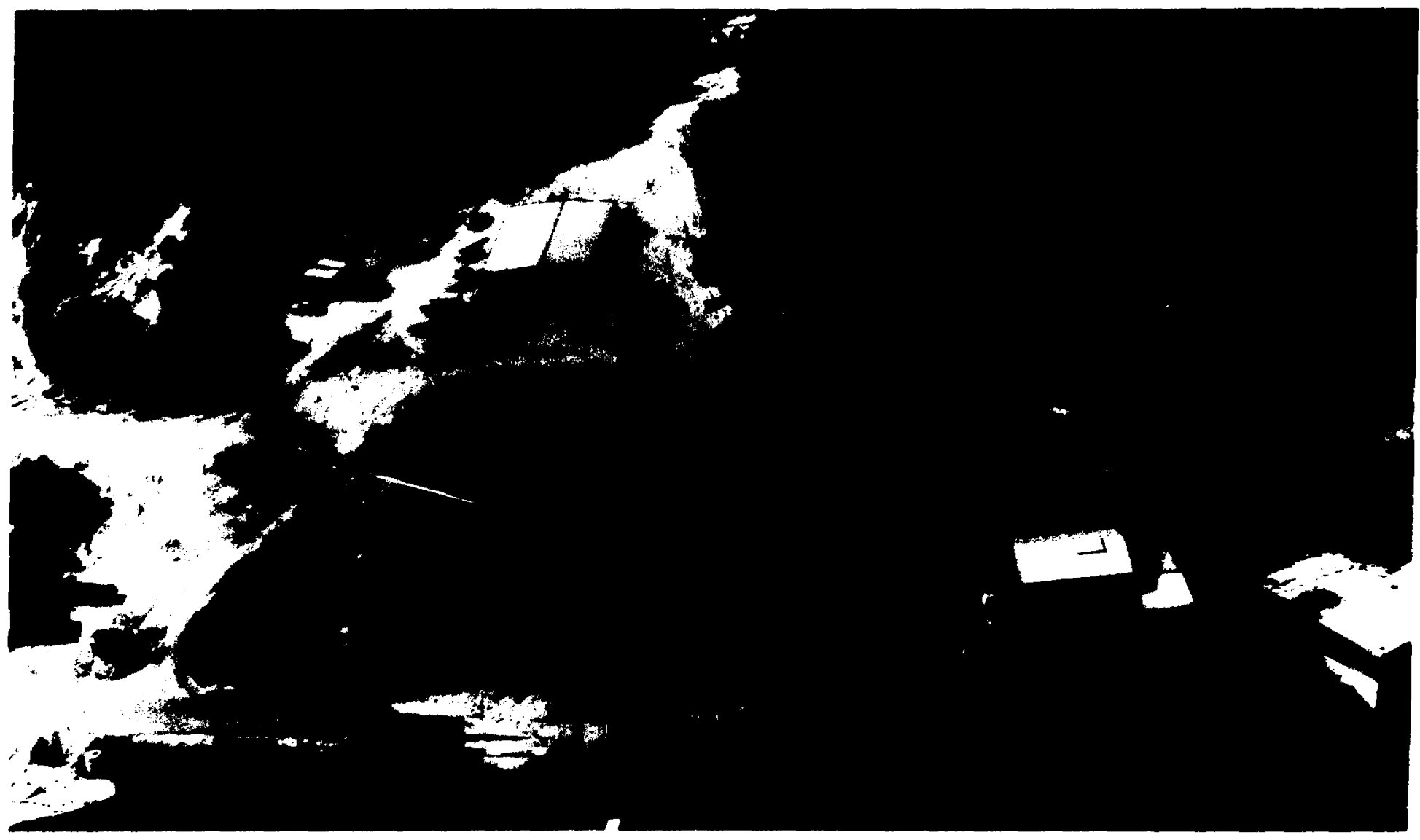

Protons for the Radiation Effects Facility (left) and the Neutral Beam Test Facility (above) come from the linac (a portion of which shows at the extreme right), which also sends protons to the Brookhaven Linear Isotope Producer (right), where isotopes are made for medical use and industry. The linac's primary function is to inject protons into the Alternating Gradient Synchrotron for physics research. 


\section{Department of Applied Science}

Research activities in the Department of Applied Science are organized into three broad areas: technology research, environmental science and energy science. Within these areas are specific divisions working on projects that range from the development of a coating system for reducing corrosion of steel surfaces, to cruises in the Atlantic Ocean for measuring the flux of materials away from the continental shelf, to studies of superconductors in order to understand and improve their properties. The following stories - one on electroresponsive polymers, the second on carbon dioxide and plant growth, and the third on injury control - further illustrate the breadth of research done in applied science.
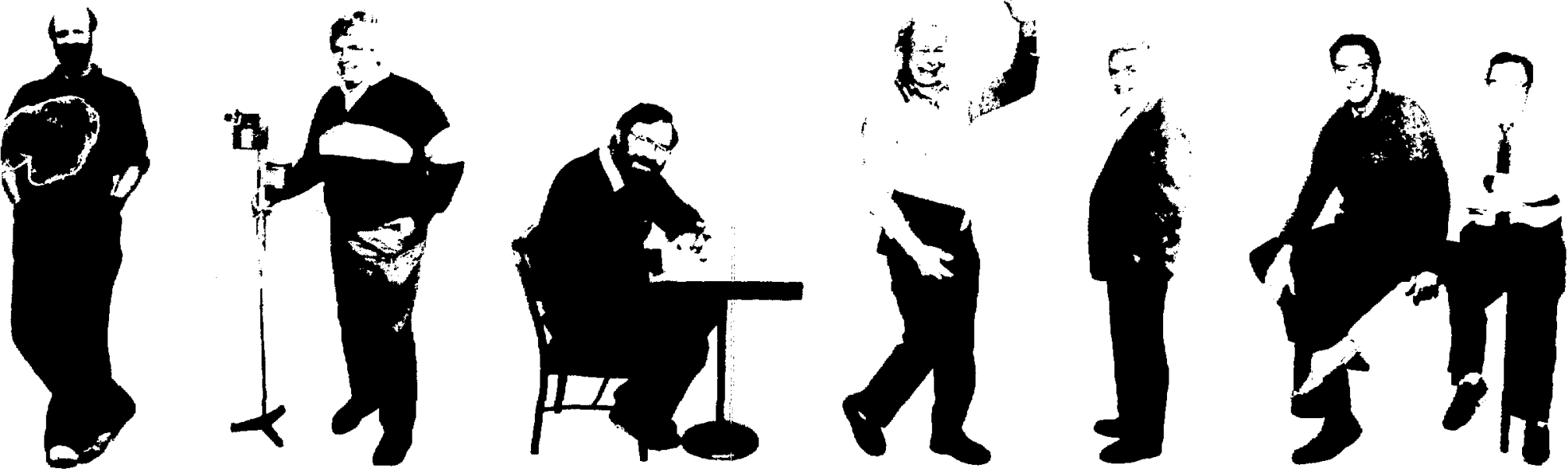


\section{Polymers for the Future}

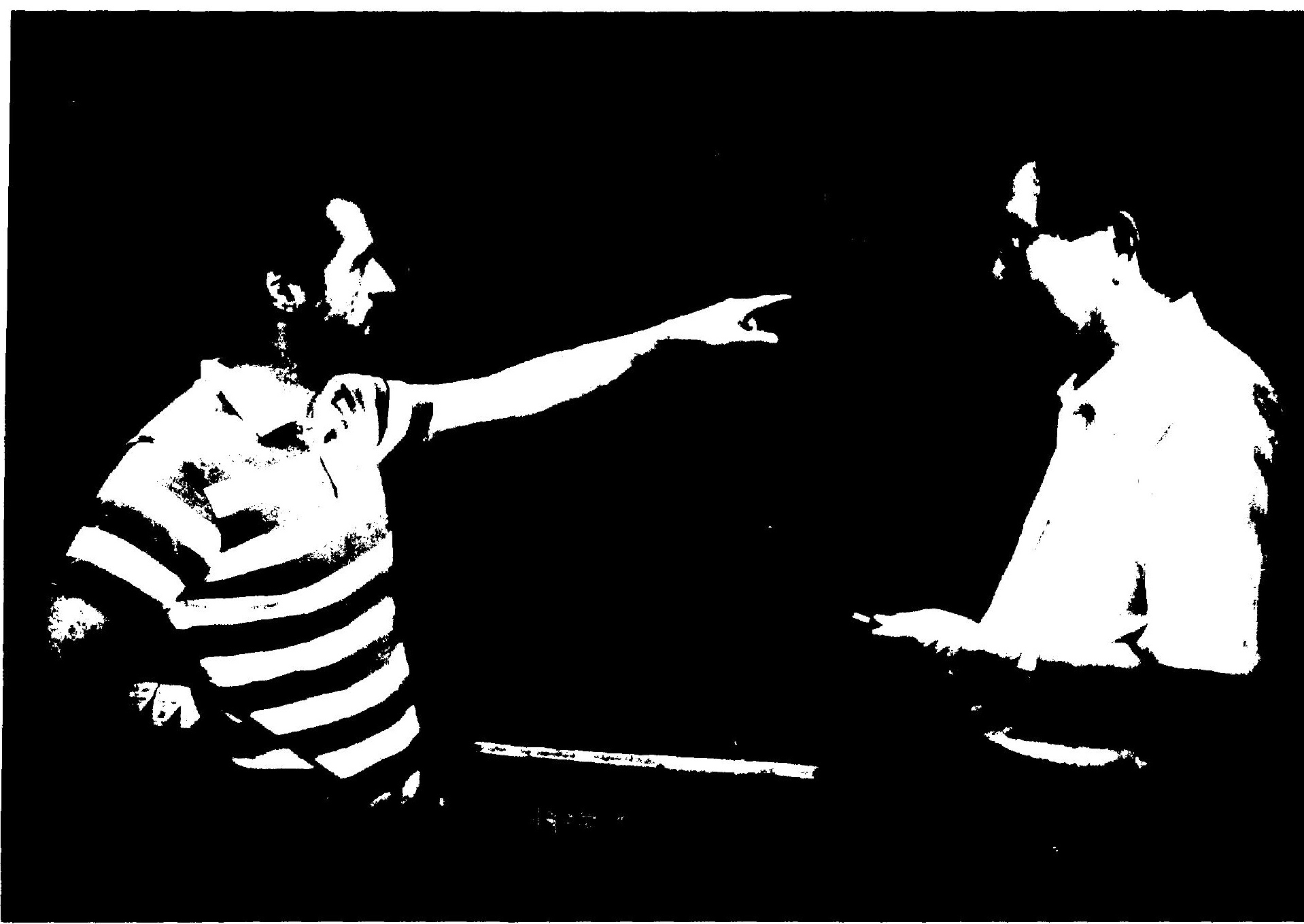

Terje Skotheim, BNL, and Yoshi Okamoto, of the Polytechnic University, discuss their collaborative work on electroresponsive polymers.

Rubber tires, silk blouses, nylon stockings and vinyl seat covers these items are examples of everyday uses of polymers. In the years ahead, polymers of a different kind - electroresponsive polymers are likely to be just as common to day-to-day living, in such objects as super batteries and optical switches.

At Brookhaven, we have been developing electroresponsive polymers for the last decade. Our latest efforts have been done in collaboration with other institutions, in particular Polytechnic:
Lniversity. Brookhaven's special contributions are in the chemical synthesis and characterization of various polymers, as well as in developing applications. Polytech focuses on synthesis and characterization.

Also important at BNL is the availability of the National Synchrotron Light source and the High Flux Beam Reactor, facilities that are well suited to the characterization of new polymers.

In dictionary language, a polymer is a molecule with a high relative molecular weight formed by the chemical linking of many simpler molecules, each of the same substance, called monomers. For example, ethylene is a simple molecule with formula:

$$
\mathrm{Cl}_{2}=\mathrm{Cll}_{2}
$$

Many molecules of et hylene string together to form polyetliylene, formula:

$$
\text { ... ( } \mathrm{CH}_{2}-\mathrm{CH}_{2}-\mathrm{CH}_{2}-\mathrm{CH}_{2} \ldots
$$

Orclinarily, a polymer is an insulator - a poor conductor of clecericit y. By doping them (adding small amounts of impurities. ass is done lo make a semiconductor), certain 
types of polymers can be made to be conducting. In an electroresponsive polymer, the electrons move in response to light or an electric field, Thus, an electroresponsive polymer can conduct electricity, be magnetic, or respond to the electric field in light waves.

Because electroresponsive polymers are synthesized, they can be tailor-made for a broad range of purposes - from biotechnology to materials science. The number of different polymers is limited only by the imagination of a good synthetic chemist. For many applications, only very small amounts of material are required, since many of the expected uses of these materials will involve thin film technology.

Electroresponsive polymers fall into three broad classes.

\section{Ion Conductors}

lon conductors are commonly made by dissolving metal salts, for example lithium salts, in specialized polymers that have the ability to bond with a metal ion. The resulting complex of metal salt and polymer becomes a solid electrolyte, in which the electricity is carried by the moving ions of the dissociated salt.

An important application of ion conductors would be in making extremely powerful, rechargeable batteries. While such batteries could certainly replace conventional batteries, they would also open the way for even broader applications, such as in making huge banks to level utility loads and in storing solar energy in the summer for later use in the winter.

A promising feature of ion conductor batteries is that they store energy with almost no draining, so they can sit on a shelf for a decade or more without losing any noticeable amount of power.

Yet another use of ion conductors would be for electrochromic windows - windows that can be made to change color by changing the electrical potential. Such windows, for example, could be controlled to stay clear on a sunny winter day and dark on a sunny summer day, thereby assisting actively in the energy management of buildings.

\section{Electronic Conductors}

Electronic conductors are polymers that have a backbone made up of alternating double and single bonds, and they can be highly conducting. We envision developing plastics that are as conductive as ordinary metals, even to the point where they can be substituted for copper wire. Conductivities as high as that of copper have already been achieved in laboratory samples.

Another area of interest would be materials that carry out specific tasks in biological systems, for example, as special sensors or for controlled drug release. Other potential uses range from connectors for severed nerves to artificial vision systems. Such applications could be achieved by implanting or growing electronic conductors directly in living tissue. The conductors, of course, would have to be biocompatible.

\section{Nonlinear Optical Systems}

When two light beams interact in a material and the interaction produces light of a different frequency, that is a nonlinear optical system.

Such systems, ones based on inorganic crystals, are already being used to double the frequency of high-powered lasers so that the light they emit can be changed from infrared to visible to ultraviolet. Conventional, small, solid-state lasers operate only in the infrared. They would be more versatile if they could emit light in a wider spectrum.

Even more promising are nonlinear optical systems based on organic materials of either polymers or crystals. These would have significant advantages over inorganic systems because the nonlinear effect is much greater.
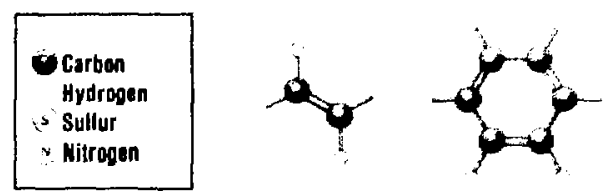

Polyacaylane
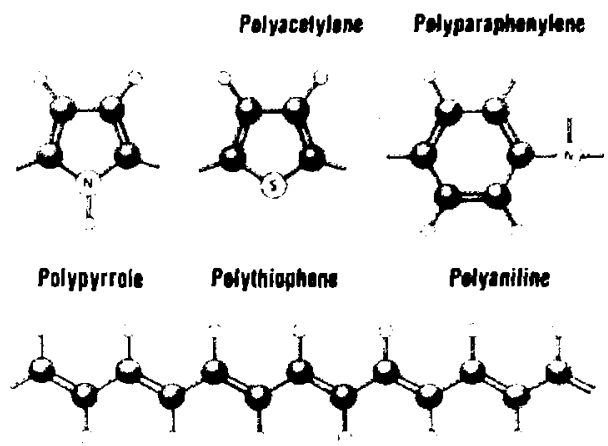

Polyacotylans Chain

Carbon, hydrogen, sulfur and nitrogen molecules can be linked together in various ways to synthesize different polymers.

For example, they could be put to use in a small solid state laser to change the frequency so that the light is more easily transmitted by optical fibers. Another application would be in the construction of optical switches, significantly faster than conventional electronic switches, which would make ultrafast computers possible. Coupled with fiber-optic technology, optical switches would open the way to dramatic improvements in the performance of telecommunications systems. 


\section{Putting the Brakes on Accidents}

When accidents happen, the victim is often blamed. But any accident may have many possible causes. A fair approach is to examine the circumstances leading to the accident, with an eye open to making changes - and not just behavioral ones that focus on the victim.

The name for this scientific specialty is injury control. It deals specifically with injury, as distinguished from illness and disease.

At Brookhaven, within the Department of Applied Science, we conduct epidemiological studies of injuries, examining such factors as their occurrence, distribution, severity, control and cost in a given population.

\section{Occupational Injuries Within DOE}

Since 1985, we have been engaged in a major study of occupational injuries within the federal government's Department of Energy (DOE) and its contractor-operated facilities, such as Brookhaven.

DOE and its contractors employ over 127,000 people who are engaged in research, engineering, production, construction and service activities. For this large population, which is like a microcosm of U.S. industry, all causes of injury will be examined for patterns through time as well as across job categories. The intent is to identify problems so that management can make systemwide policy decisions, such as where to spend money to reduce risk of injury.

Initially, our efforts have been concentrated on gathering data on injuries. Data are being taken from the point of origin - the form filled out in the medical department, dispensary or clinic, in which a physician or health professional states a diagnosis of injury. Follow-up reports are also being examined to obtain such statistics as hospitalization and number of workdays lost.

Because information is collected and interpreted differently among DOE contractors, we have had to introduce a standardized coding system of cause and type of injury, based on an internationally accepted procedure. We have also had to incorporate a method of measuring severity of anatomical damage, based on modifications to a widely-accepted injury scaling procedure that quantifies damage from the most minor injury to those that are life-threatening or untreatable.

Generating the data base is a monumental task, but once done, it will be the first of its kind. The data base will be used to identify areas in need of attention, which then can be put into an order of priority. The next step will be to select one area for a pilot intervention program. If proven effective, the pilot program will be implemented throughout the DOE system. And that would be just the beginning of perhaps the broadest injury control program in any heterogeneous organization in the country.

In 1985, the National Academy of Sciences (NAS), at the direction of Congress, embarked on a review of injury in the U.S. The latest NAS report on the topic, released in 1987 , expressed concerns that reporting of occupational injuries in the U.S. was inaccurate and unreliable. We at Brookhaven were pleased to note that we had already incorporated the NAS recommendations in our ongoing DOE protocol, further assurance that the results of our work will have far-reaching benefits.

\section{New York's Seat-Belt Law}

Separate from the DOE study, Brookhaven is studying motor vehicle crashes in New York's Suffolk County, as part of a project to evaluate the state's seat-belt use law. The work is being done in collabora-

\section{Teaching Injury Control in the Schools}

Investigations of major disasters indicate that injury control is not always sufficiently addressed in the design, operation and maintenance of machines, complex high technology systems, buildings, or entire facilities. While engineers add in standard margins of safety when designing something, they are generally trying to prevent failure. It is also important to design something so that when it fails, it will do so in a way that minimizes death or injury.

One way to do this is to go back to basics - to go to the schools.

At present, engineering schools require no formal coursework in injury control. Brookhaven has recommended to the Accreditation Board for Engineering and
Technology, which defines the curriculum in engineering schools, that a thorough grounding in injury control be given all engineering students. This could produce a new generation of engineers who, when designing a system for a new technology or modifying existing technology, will build in safety from the beginning. 


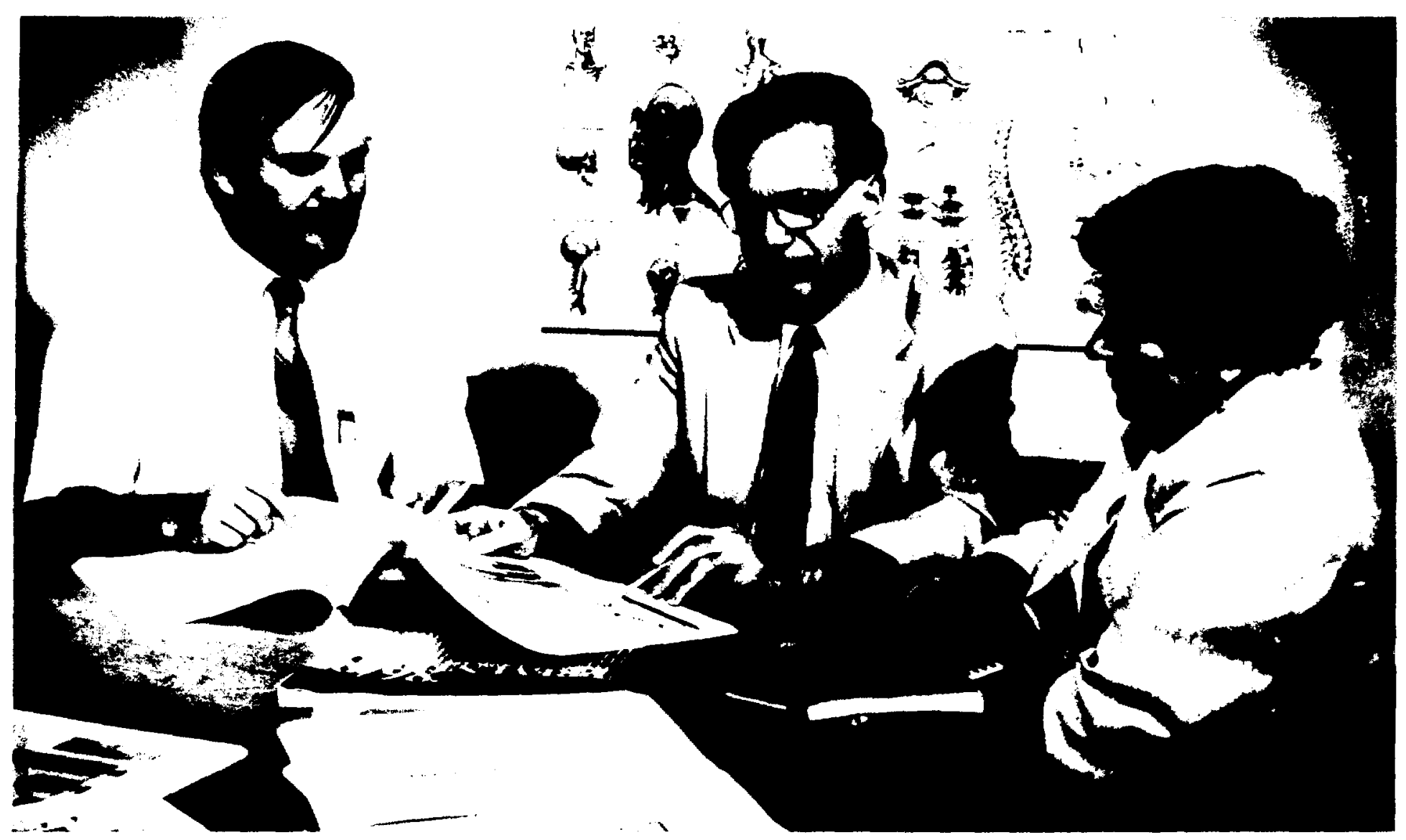

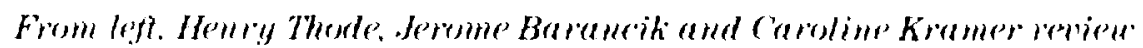

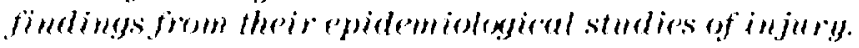

tion with the State I niversity af vew Fork at Stony Brook, and it is the first full medical evaluation of' the efferets of the law.

New Yorkis seat-belt law, which recpuires that oceupants in the front seat of a cal wear seat belts, went into effect Janualy 1, 1985. During the first half of that year, the number of deaths due 10 motor vehiclo rashes in Suffolk ('ounty was 24 pereent lower than in the same periol the year before. We think the difference is related to compliance with the latw.

Data foom hospital and physician records of relnicular injuries show that the sharp increase in seat-belt usage in 1985, particularly in the first quarter correlates with fewer fat al and nonfatal injuries. In adodition. berause of our very sensitive analyt iral techniques. we have disroverect a pattern of injury that otherwise would have been missod:
Head injuries decreased by over 2.2 pereent. while nose and jaw fracfures doubled. This suggest s that heads may be hitting windshields, sterering wheels and dashboards less often, a subject for further study'.

Seat-belt laws have been repealed in Massarehusetts and Neblaska. and seven of the 24 states with these lass still in effect are considering repeals. In light of this, 13 rookhavents findings are espectially timely:

Planned for the future is an extension of our Stony Brook collaboration: a project on threshold brain injury (concussions) and neck and resvieal spine injury. l sing measurements taken at actual motor vehicle cash semes, as wedl as sophisticatcol moclical imaging torelmicues. we experet to irlentify the specifice parameters that ratese roncussions. This wrok shomkl lead to bottere alesign of problection goall such as shoulder lap rest raint sistems, helmets and passenger compartment interiors.

\section{The Key Is Prevention}

Most people worry that their productive years will be cut short by such healt h problems as cancer or heart disease. The lact is, one's productivity is most likely to be lost through injury.

In injury control, the key is prewontion - before and eson afier an injury-producing event lakes place. Brookhaven is adolressing major comerens in both orecupational health and public hoalth, and wo (xperet that our work will lead to better prevent at ive measures in botll aremas. 


\section{The Greenhouse Effect: About FACE}

G ing in recent years, leading to speculation about such effects as higher sea levels from melting polar ice. Some scientists believe that the cause is pollution in the atmosphere. If this is true, then the global warming, sometimes referred to as the "greenhouse effect," may be yet another example of how humankind has drastically altered life on earth.

The primary pollutant is carbon dioxide $\left(\mathrm{CO}_{2}\right)$, released in the burning of coal, oil and gas. Levels of $\mathrm{CO}_{2}$ in the atmosphere have risen from 280 parts per million ( $\mathrm{ppm}$ ) in the mid- 18 th century, to $316 \mathrm{ppm}$ in 1958 , to 345 ppm in 1985 , and scientists forecast a doubling of the current level some time in the next century.

At Brookhaven, one of our research programs on carbon dioxide enrichment focuses on the feedback of $\mathrm{CO}_{2}$ into the plant system.

In nature, $\mathrm{CO}_{2}$ is released into the atmosphere from decomposing organic matter and taken up by plants during photosynthesis. Scientists speculate that increased global warming may accelerate decomposition, therefore increasing the flux of $\mathrm{CO}_{2}$ from natural biological systems into the atmosphere. At the same time, because of enhanced photosynthesis, natural areas such as forests and tundra may also remove an increased amount of the $\mathrm{CO}_{2}$ emitted from man-made sources each year. For agricultural crops, some studies have indicated that a doubling of $\mathrm{CO}_{2}$ will have the positive effect of increasing plant yield by as much as 50 percent. In general, however, few long-term studies have been done to examine the impact of increased $\mathrm{CO}_{2}$ on either natural ecosystems or agricultural crops.

In 1986, Brookhaven developed a unique plant exposure system that

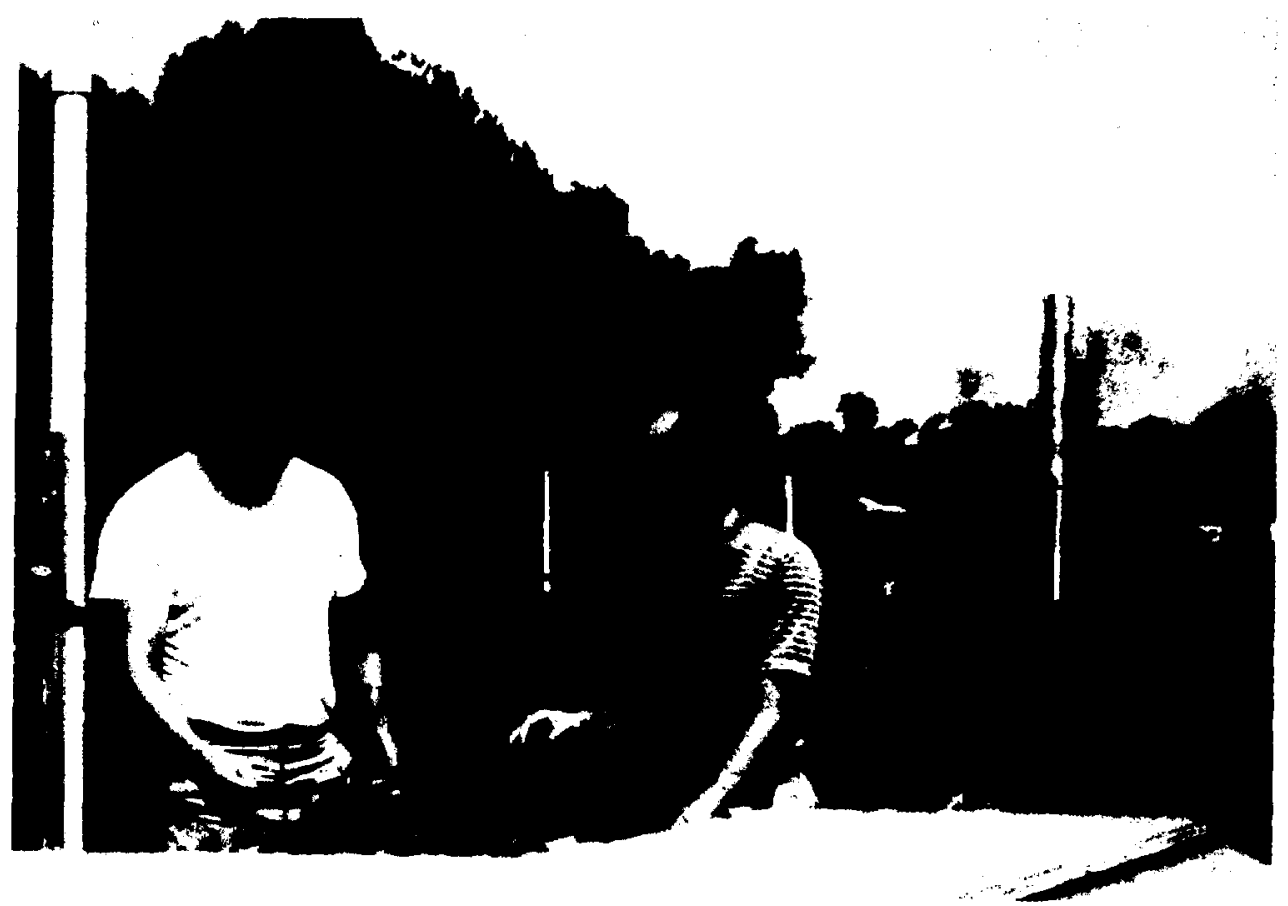

In Yazoo City, Mississippi, Lance Evans (left), of Manhattan College, and BNL's Keith Lewin examine the leaves of cotton plants grown within a Brookhavendeveloped exposure system for a study of the effects of carbon dioxide enrichment on crop yield.

allows the study of plants in an open field. Called FACE, for free air controlled enrichment, the system was designed in short order, largely due to the multidisciplinary nature of Brookhaven, where experts in various areas can be called upon for quick advice or assistance. In addition, the Laboratory has a long history of developing and working with various types of plant exposure systems.

The FACE system is composed of a circular array of 32 individually valved, vertical pipes connected to a toroidal chamber. A high-volume blower injects ambient air into the chamber, where it is mixed with elevated levels of $\mathrm{CO}_{2}$. The release of $\mathrm{CO}_{2}$ is controlled by a computer system that measures wind direction, speed and the $\mathrm{CO}_{2}$ concentration in the center of the array, and uses this information to adjust the rate of gas to be released from the verti- cal pipes on a second-to-second basis. Hence, natural microclimatological mixing processes are used to disperse the gas, while a computercontrolled gas release system maintains a desired concentration. The resulting environment is as close as practical to reality.

In the first year, we tested the system with a 15-meter diameter prototype, using sulfur dioxide as a test gas in a grassy field. It worked so well that we were ready for a field experiment the following year.

In 1987, then, we took our operation to a cotton field in Yazoo City, Mississippi, joining forces with a number of other institutions to study effects of $\mathrm{CO}_{2}$ enrichment on crops.

Yazoo City has a large ammonia factory, the Mississippi Chemical Company, which generates $\mathrm{CO}_{2}$ as a by-product. In a prime example of industrial cooperation, the company 
agreed to supply the $\mathrm{CO}_{2}$ needed for the study and also allowed the project to use a 20 -acre commercial cotton field, which it owns.

For this first year in the field, we built a 22-meter array and studied the system's ability to control gas concentrations. Using four tons of $\mathrm{CO}_{2}$ per day, the system was successful at holding concentrations of 500,600 and $700 \mathrm{ppm}$.

In 1988, we assembled four arrays in the cotton field and used up to twelve tons of $\mathrm{CO}_{2}$ per day to expose the plants to a concentration of 500 $\mathrm{ppm}$. One objective of this latest field work was to evaluate how well a multi-array system adjusts to gas distribution interference. In other words, when the gas drifts from one array to another, the control system must throttle back in the second array in order to maintain even exposure. Preliminary results show that each array is able to adjust to the "leakage" of $\mathrm{CO}_{2}$ received from neighboring arrays.

So far, the system is performing well. Studies conducted at other labs in greenhouses and chambers indicate that a doubling of $\mathrm{CO}_{2}$ will increase cotton growth by about 80 percent, with a concurrent decrease of about 50 percent in water demand by the cotton plants. It may be that plants grown in enhanced levels of $\mathrm{CO}_{2}$ will have increased tolerance to drought (which may be aggravated in the first place by the increase in $\mathrm{CO}_{2}$ ).

In the future, we hope to do a fullscale field experiment, using up to 12 FACE arrays and up to 50 tons of $\mathrm{CO}_{2}$ per day. When we are confident that the system works with a single crop, the next step will be to evaluate effects of $\mathrm{CO}_{2}$ enrichment on a natural community of plants, such as tundra, forest regrowth and grazing lands.

The great advantage of the BNL system is that it comes closest to providing a natural environment for plants. Although numerous studies have been done on plants grown in

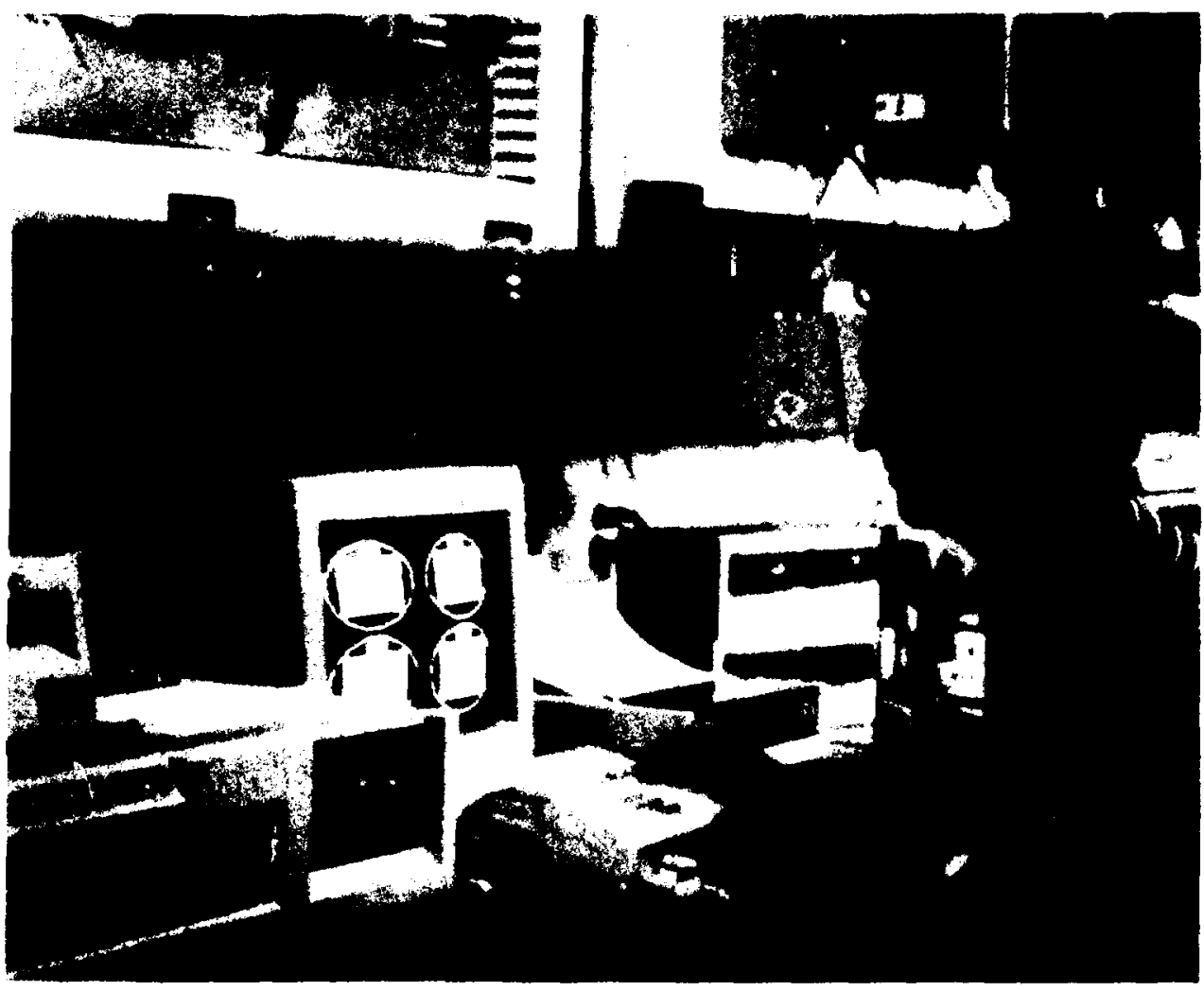

Keith Lewin sits at the computer that operates the carbon dioxide exposure system, which consists of four arrays of vertical pipes, through which carbon dioxide-enriched air is emitted. The computer monitors wind speed, wind direction and carbon dioxide concentration within the arrays and decides where and how much gas should be emitted.

enclosures, such as growth chambers and greenhouses, those plants are different from plants grown in open fields. They don't look the same, and their growth characteristics are different. No matter how cleverly designed, enclosures alter the microclimate, temperature, light and evapotranspiration conditions. One is never really sure that data obtained from studies of enclosed plants are a reasonable representation of what would happen in nature.

An added benefit is the FACE system's mobility. All of the gas analysis instrumentation, the electronic control system, the system control and data display computers, the climatological monitoring systems and the air quality analysis instrumentation are mounted in a trailer. Thus, the system is capable of moving to different locations in order to study various crops and ecosystems. 


\section{National Synchrotron Light Source Department}

The world's foremost facility for scientific research using $\mathrm{x}$-ray and ultraviolet radiation is operated by the National Synchrotron Light Source (NSLS)

Department. In a single year, about 1,000 researchers are sent from 163 separate institutions to use this "best and brightest" source of synchrotron light. Often in collaborative teams with NSLS scientists, they perform a wide range of innovative experiments in physics, chemistry, biology, materials sciences and various technologies.
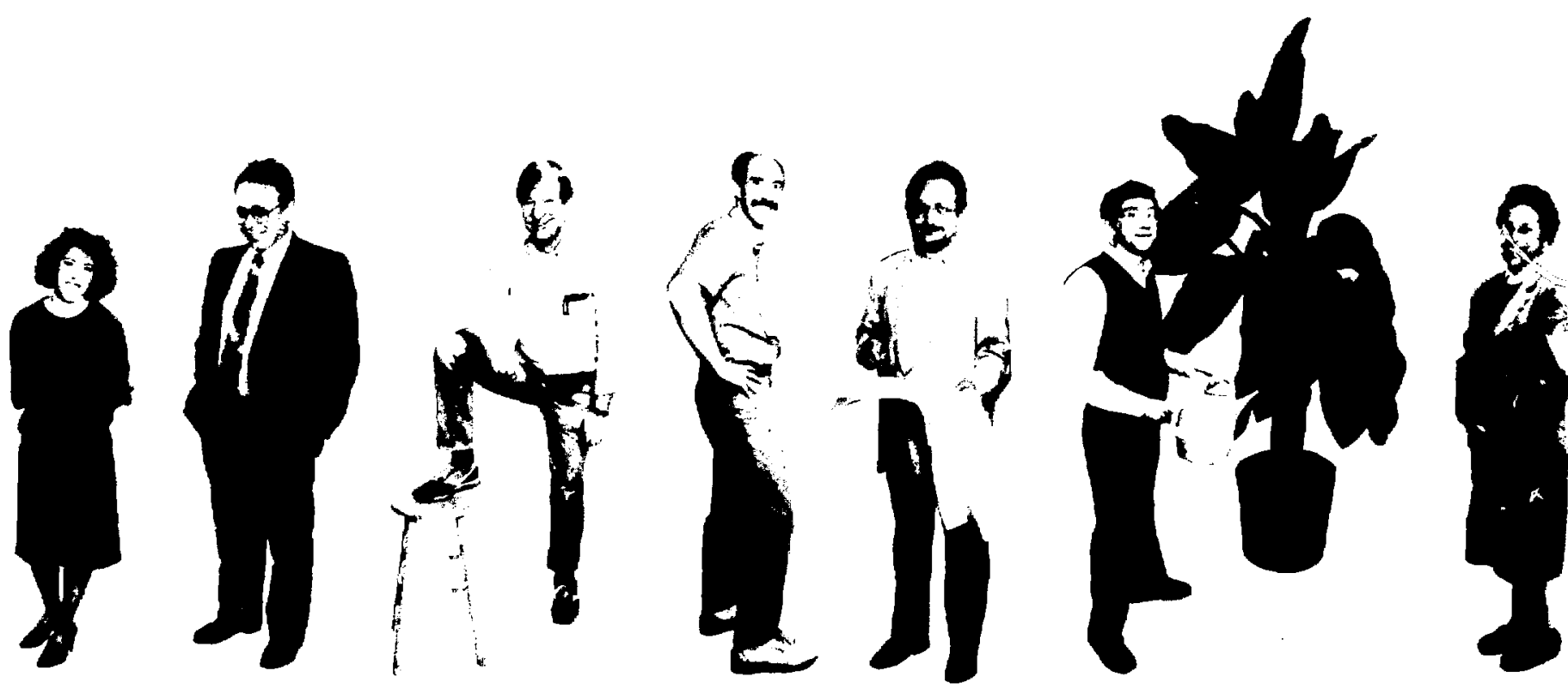


\section{Breakthrough in Chip Technology}

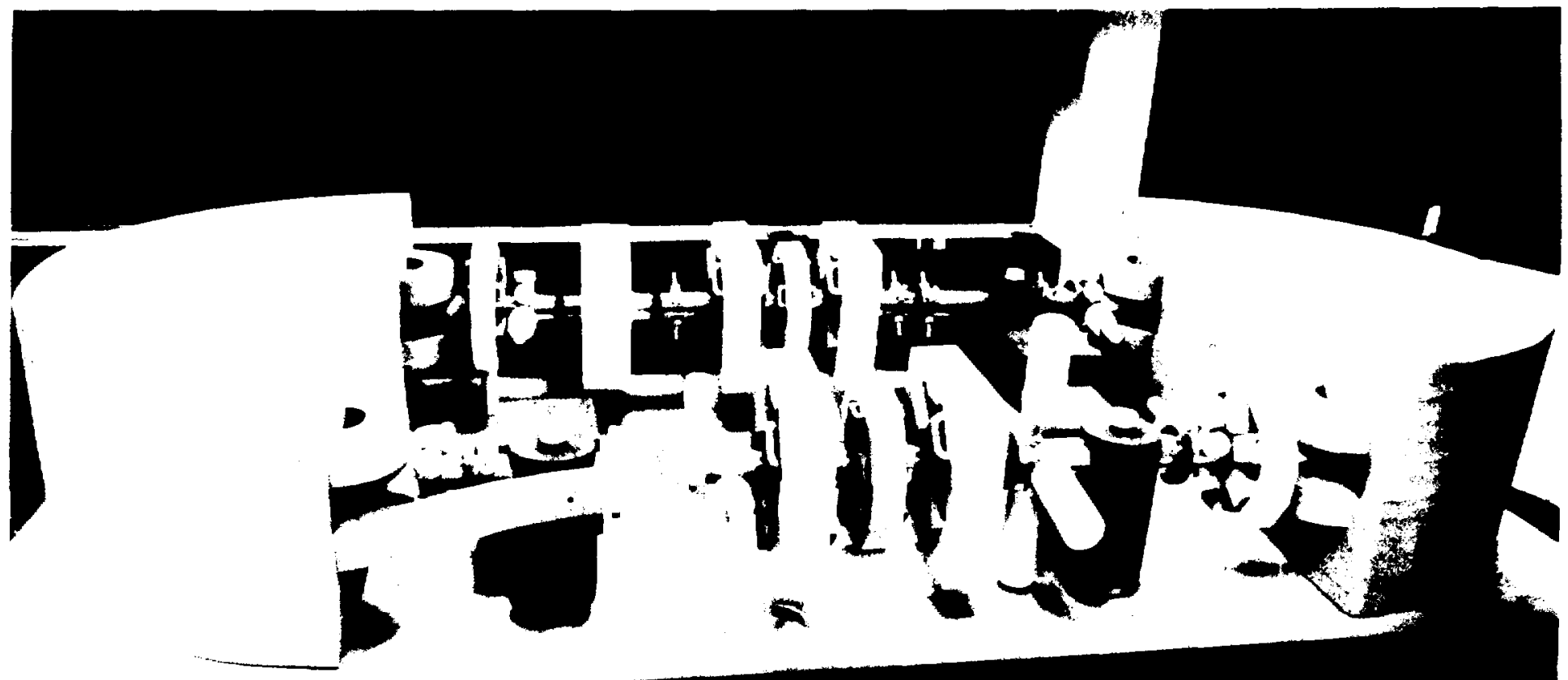

A full-scale model of the first United States compact synchrotron. Designed and built at the National Synch rotron Light Source, the synchrotron is 8.5 meters in circumference and uruld fit comfortably in a normal living room.

Sometimes, bigger is bet ter - but not in the case of computer chips. The smaller the spacing between its components, the denser the chip, and the shorter the distance that information must travel. So the smallest chips team together to form the fastest, most powerful computers.

In May 1988, at Brookhaven's National Synchrotron Light Source (NSLS), some of the most densely packed, working computer chips in the world were completed by a team of researchers from International Business Machines Corporation (IBM).

Stationed at the NSls vacuum ult ra-violet ( $(\tau \mathrm{V})$ ring, the scientists made test chips with only 0.5 micron spaces between components. One micron is about one hundredth of the width of a human hair, mak- ing the new chips two to three times denser than any others available.

The new chips were a breakthrough - and not only because of their increased capacity. They also demonstrated the effectiveness of a new potential manufacturing technique: $x$-ray lithography.

$X$-ray lithography works much like photo development, in which light is beamed through a negative to produce a positive image on a print. The $x$-rays are beamed through a series of very thin masks, which hold the intricate image of the chip's circuits. The image is transferred by the $x$-rays onto a photoresist layer, attached to the top of a silicon wafer.

After extensive processing, which includes baking, etching and washing, the circuit image is event ually etched into the wafer. The wafer is sliced into individual chips, which are then inserted into a package with other electronic parts.

Not all $x$-rays are suitable for $x$ ray lithography. "Hard" $x$-rays, used most often in medicine, pass right through the image on the masks. The VUV ring's soft $x$-rays have proved to have the right wavelength. They give the $x$-ray technique several advantages over optical lithography, the present method of using ordinary light to make chips for microcircuits.

The most important of these advantages, of course, is in being able to produce circuits and features one-tenth of a micron wide against optical lithography's present manufacturing standard of eighttenths of a micron wide.

Also, unlike the ultraviolet light used in conventional methods, $x$ rays can pass through the mask 10 the nearby wafer with no need of focusing. This avoids distortion caused by focusing lenses and allows a larger area of the silicon 
wafer to be exposed. Larger chips can therefore be easily fabricated.

As a third "plus," $x$-rays can pass through tiny particles of dust that might cause defects. Defects are a major cause of lower yields in existing chips.

Since the NSLS is the workplace for the latest scientific and technological advances in this field, Brookhaven has become a leader in a national effort to develop a new, smaller ring dedicated to advancing $\mathrm{x}$-ray lithography. Brookhaven's goal is to transfer the compact synchrotron technology to industry so that by the mid-1990's, circuit components can begin to be manufactured in the United States.

In looking ahead at the computer industry's manufacturing needs, it could be envisioned that a facility established at a large synchrotron like the NSLS could produce as many as 20 or more wafers an hour at each of the 90 beam lines. Such synchrotrons are too large for a single firm to be able to maintain, however. Also, sharing by manufacturers with differing production methods would often be impractical.

One answer to the problem would lie in the use of compact synchrotrons, which are room-size, costeffective synchrotrons using supercondurting magnets. Firms could afford to own and maintain them independently, and convenient siting and specialized layout could increase production.

The federal Department of Defense has funded Brookhaven to help establish domestic vendors of compact synchrotrons. To do this, the NSLS is building the first compact synchrotron, then will transfer the prototype technology to industry. The resulting switch to high technology production would bring America's computer chip industry to the forefront of world competition.

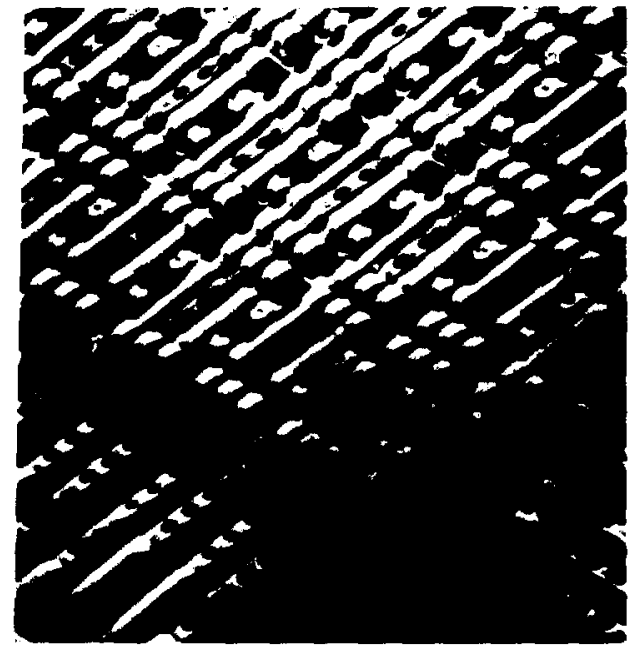

One of the most tightly packed, uxorking computer chips in the urorld, as magnified under an electron microscope, made by IBM at the National Synchrotron Light Source. Transistors are buried beneath the metal connection lines, which are less than one micron wide, or about one-hundredth the width of a human hair. The metal lines connect to the transistors at the small, dark circles, which are 0.5 microns in diameter.

\section{Orbits, Spins and Resonance: An Elegant Experiment}

$\mathrm{T}$ signals were large and the experiments had produced useful results. Then, when the same techniques were tried on a similar sample, the signals were surprisingly small. What was happening?

At Brookhaven's National Synchrotron Light Source (NSLS), this was one of the questions confronting physicists investigating the magnetic properties of certain rare earth metals. The collaborative effort ultimately included scientists from Brookhaven, AT\&T Bell Labs, Cornell University, Rice University and the Institut Laue-Langevin in France.

This part of the research began at Brookhaven in 1986. Then, while the $x$-ray ring of the NSLS was shut down for modifications during 1987 , the researchers carried on their experiments using an $x$-ray beam at the Cornell High Energy Synchrotron Source at Cornell University.

$\mathrm{X}$-rays are sensitive to a sample's magnetic structure. Because they are part of the electromagnetic spectrum, $x$-rays interact with and are scattered by particles. The signals, however, are weak unless induced by a powerful synchrotron radiation beam. The very high resolution of synchrotron radiation has the potential to reveal magnetic structures more accurately than ever before.

Over the past four years, several research groups have used $x$-rays from synchrotron sources to investigate magnetic effects. Examples include the spiral structures of the rare earth metals holmium and erbium, magnetic transitions in disordered magnetic systems, and magnetism in multilayer thin films.

More recently, in an extension of their original techniques, the researchers began to use the linear polarization of the synchrotron $x$ ray beam in order to study the polarization dependence of the scattered beam.

An x-ray beam is a very short wavelength light beam made up of propagating (that is, traveling) electric and magnetic field vectors (directions) that are oriented at right angles to each other. The direction of travel is perpendicular to both of these vectors. In a linearly polarized $x$-ray beam, the electric field vectors are all lined up with each other.

The researchers directed a linearly polarized beam onto a holmiun target, which scattered the beam. Then they measured the lin- 
ear polarization of the scattering, which means that they measured the intensity of the scattered beam, both parallel and perpendicular to the diffraction plane. The diffraction plane is the flat surface formed by the incident, or incoming, and scattered $\mathrm{x}$-ray beams.

To understand this experiment better, it is necessary to know something about atoms and their magnetic moments. An atom has a nucleus that is surrounded by moving electrons. It also has a "magnetic moment," made up of two components: orbit and spin.

Spin refers to the intrinsic moment of the electrons, as if each electron were rotating on its own axis. The orbital moment refers to the circulation of the electrons within the atom. One way to picture this is to think of a circus rider making pirouettes on a horse's back that's spin. The orbit would be represented by the horse as it gallops around the ring.

When the researchers analyzed the data from the holmium experiment, they found that the parallel scattering was proportional to a value that depended on the orbital plus spin components of the atom's magnetic moment, while the perpendicular scattering was proportional to a value dependent on just the spin component.

Thus, with a polarized beam, the spin and orbital contributions to an atom's magnetic moment could be identified and characterized in a direct experiment, something that had not been possible before.

But the mystery was not yet solved.

During the experiment, the beam was tuned to different energies. At certain energies, there was a sudden "resonance" - the magnetic signals became about 50 times stronger.

These resonances occurred at energies corresponding to absorption edges. Absorption edges in a solid are the energies at which the absorption of incoming light abrubtly changes.

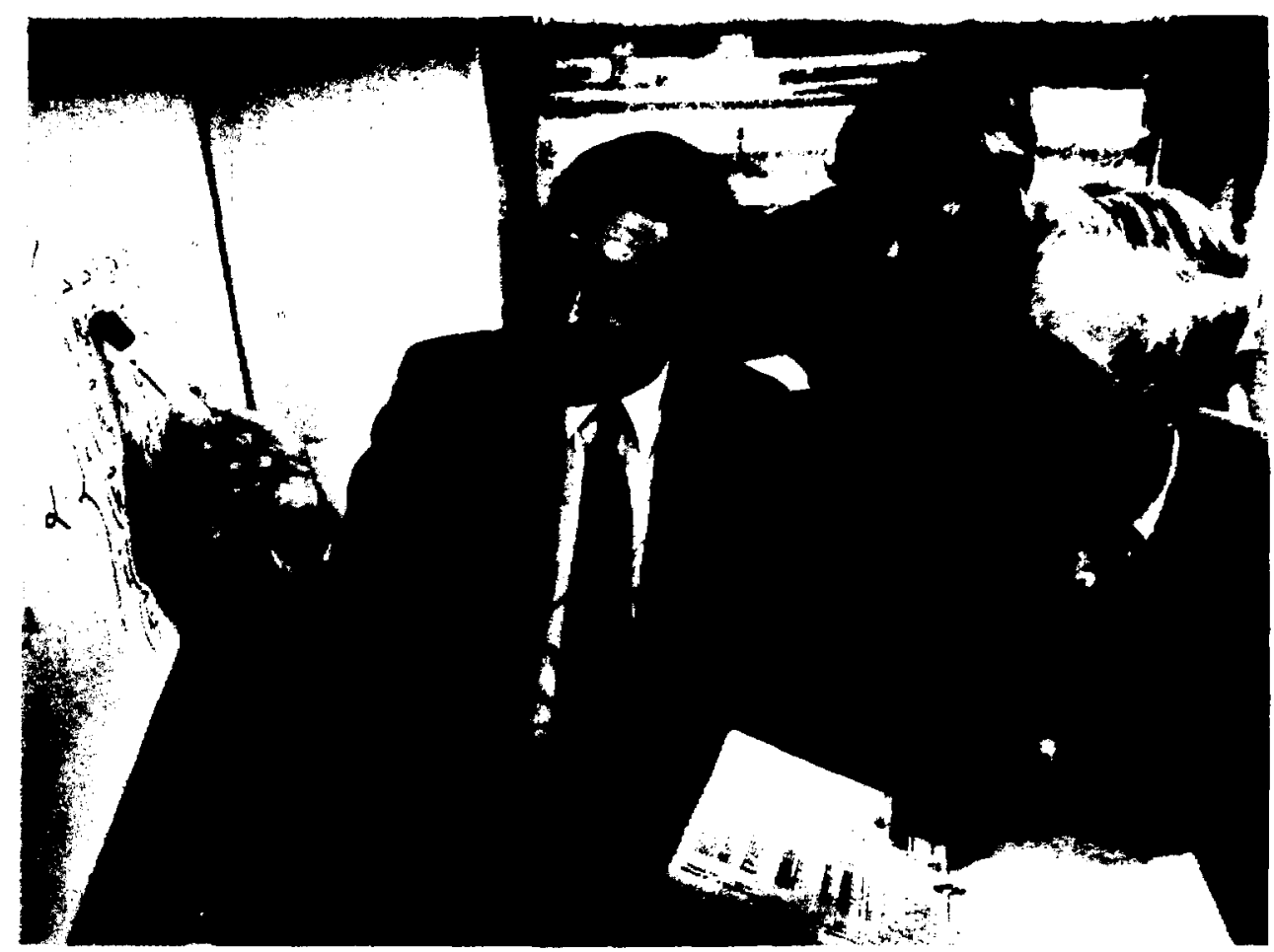

(From left) Brookhaven's Martin Blume and Doon Gibbs discuss with Denis McWhan, AT\&T Bell Labs, the theoretical aspect of their experiments on the magnetic properties of rare earths. Further research will be carried on at the National Synchrotron Light Source, the white building visible through the urindow.

The experiment showed that when the beam's energy was tuned to these energies that matched the absorption edges of holmium, a resonance occurred, with a resultant dramatic increase in the magnetic scattering.

This increase at the absorption edge can explain one of the experiment's original puzzles - why one material produced a large signal while a similar material produced a small one. The first material had been studied at $x$-ray energies near to its absorption edge, while the second had been studied far above its edge.

This resonance behavior tells us in new detail about the magnetic states in a solid. One important result of the discovery is that the technique may be used to explore materials such as the new hightemperature superconductors, whose magnetic signals were believed too weak to be studied by $\mathrm{x}$-rays.
The researchers have performed a single experiment - that of using synchrotron $x$-rays to study magnetic properties of solids - to produce two different results never before obtained, simply by tuning the energy of the beam and measuring its polarization when scattered. When the x-rays' energy is not at the sample's absorption edges, the orbit and spin of the sample can be separated; when a resonance is observed, information as to the magnetic states of solids is obtained.

Scientists view an "elegant" experiment as satisfying both intellectual and aesthetic criteria. In this experiment, the polarization dependence of the scattered beam is measured both on and off resonance. Different kinds of information were obtained each time - and this qualifies the experiment as elegant. 


\section{Innovative Optics at the Light Source} The brightest synchrotron light in the world shines at Brookhaven's National Synchrotron Light Source (NSLS), drawing an international community of scientists to perform experiments here. The swift pace of research leads to rapid advances in technology - and optical instruments at the NSLS exemplify the innovations achieved.

Synchrotron light is the electromagnetic radiation given off by electrons when they move in a curved path at close to the speed of light. The radiation, carried by units of light called photons, is very bright, enabling researchers to see minute samples of material at extraordinarily high resolution.

Like all light, the radiation comes in varying wavelengths. Experiments at the NSLS use mainly $x$-rays and ultraviolet rays, each with their own wavelength range. Optical de-

\begin{abstract}
vices called monochromators are used to isoiate a narrow portion of the light, so that only photons of a certain wavelength will pass through them. Then an arrangement of mirrors can be used to focus the selected photons into a small spot. This fine, uniform beam scatters when it hits a target sample, and the scattering pattern gives information about the atoms in the sample.
\end{abstract}

\section{Mössbauer Monochromator}

The Mössbauer monochromator was recently developed for a nuclear physics experiment that is exploring a phenomenon called nuclear Bragg scattering. Radiation striking a crystal is strongly deflected only at certain angles, called the Bragg angles. These angles depend on the arrangement of atoms in the crystal.

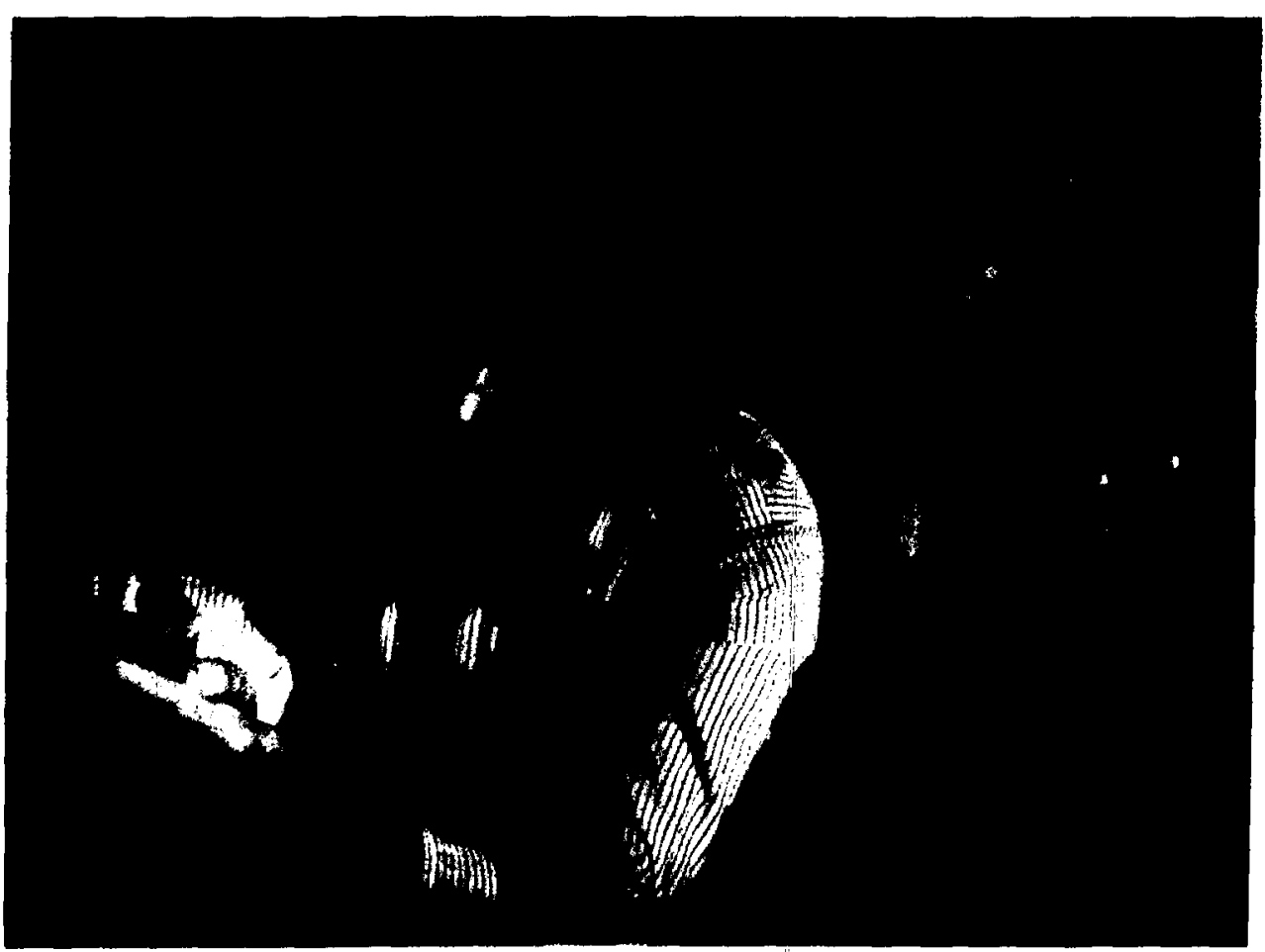

Peter Siddons (Refl) and Jerome Hastings are examining the hybrid wiggler, socm to be instalied on beam line X21, that gives the extra intersity needed for. their experiments with the high resolution inelastic scattering spectrometer.

Usually, $x$-ray scattering comes from the electrons that surround the nucleus in an atom. The nucleus itself does produce scattering, but it is normally 10,000 times weaker than that from the electrons and goes unnoticed.

For some special atoms, however, nuclear scattering is easier to see at a very specific wavelength. This is called the Mössbauer effect. By including these atoms in a carefully chosen crystal structure, it is also possible to suppress the scattering by electrons. Under such circumstances, one can observe Bragg diffraction arising entirely from the nucleus.

This scattered radiation has some interesting properties. For example, it is extraordinarily concentrated, making it perhaps the most intense monochromatic photon source available.

Although synchrotron radiation gives such intense light, a problem in using it to generate this beam is that the symchrotron produces a very broad range of energies. On this scale, a conventional monochromator produces too broad an energy band. Consequently, many of the photons striking the sample merely confuse the issue.

Brookhaven scientists solved this problem by designing a new monochromator with an ultrahigh energy resolution about one thousand times better than conventional designs. Thus, new information about nuclear Bragg scattering systems was obtained, and the way was opened toward further applications of the new monochromator.

\section{"Dragon" Monochromator}

Another new monochromator is nicknamed the "Dragon" from the long, dragon-like shape of its beamline. Designed and built by a team from AT\&T Bell Labs working at the 
NSLS, the Dragon can single out photons that fall in the "soft $x$-ray" region of the electromagnetic spectrum. Rays in this region have longer wavelengths than those of other $x$-rays. They form one of the most difficult spectral ranges to monochromatize, especially to the unprecedented standard of spectral resolution found in the Dragon.

When the beam hits the apparatus, a high precision spherical grating spreads out the soft $x$-rays. A narrow, spectrally pure band of photon wavelengths can then be selected for the experiment. The excellent performance of this monochromator is achieved by coupling two focusing spherical mirrors and a movable exit slit to the grating in a unique and novel way that totally eliminates optical distortions and aberrations.

The very fine, highly resolved beam of radiation that emerges from the Dragon is most useful for studying electronic and geometric properties of materials on an atomic scale. Among other research projects, the Dragon is now breathing its illuminating fire into investigations of the new high-temperature superconductors.

\section{New Wigglers}

Other innovative optical instruments at the NSLS are the threecrystal, high-energy diffractometer and the high resolution/high intensity inelastic scattering spectrometer. Each of these incorporates a monochromator and each depends on one of the new wigglers that have just been installed at the NSLS. Wigglers are magnetic devices that live up to their name: They wiggle the electrons in different ways to change the energy of the light beam.

One of the new wigglers uses powerful superconducting magnets to raise the energy of the NSLS photons to 150 kilovolts, about ten times higher than usual. This is the energy level needed by the threecrystal, high energy diffractometer. To learn about the structure of a sample, this apparatus isolates a wavelength of the high-energy light, directs it on a target, then measures the directions in which it is scat- tered by the target. The extra-high energy beam can penetrate a sample of steel one centimeter thick, 20 times deeper than before, and data show up much more strongly.

The high resolution/high intensity spectrometer relies on another wiggler, the hybrid wiggler. This device makes the beam curve in many little bends. Each bend produces a similar intensity to that given by a normal beam line, and so the wiggler gives an intensity about 30 times greater than normal.

This high intensity beam will be used to perform experiments that are impractical on standard beam lines. In particular, a monochromator is being developed to probe $x$ ray energy transfers of a few parts per million. The new instrument produces a highly monochromatic, focused beam that is scattered by the sample, going through minute energy changes in the process. Scientists then analyze the details of these energy changes, gaining a deeper understanding of low energy excitations in solid samples. 


\section{The Energetic Molecules}

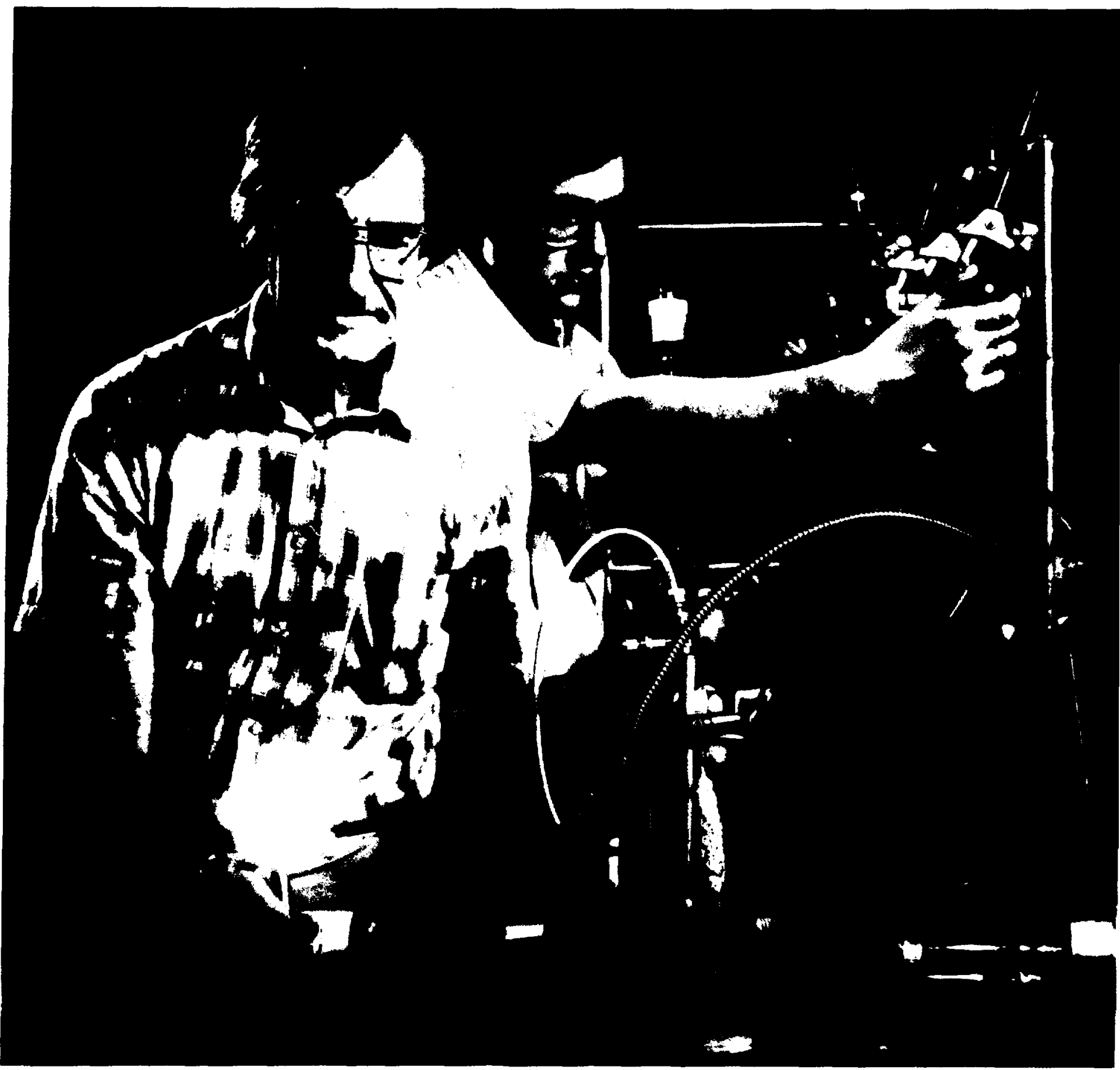

Ralph Weston (eft) and James McAndrew are aligning their apparatus, using a visible light source. instead of the laser used in an actual experiment. 


\section{Bend and stretch!}

\section{Bend and s-t-r-e-t-c-h!}

Are these the instructions at an exercise class? They could be. Or, they could be a description of the way molecules behave when energy is transferred to them in certain collision processes being studied by scientists in the Chemistry Department.

A molecule can be excited in its lowest energy state, which is called its ground state, by absorbing light or by colliding with another molecule. If the excitation is sufficient, chemical rearrangement or fragmentation may occur.

Competing with this chemical reaction is energy loss by exactly the reverse of the excitation process that is, the energized molecule emits light or bumps into another molecule. Hence, when two molecules collide, the energy transfers that occur play an important role in many chemical reactions.

Chemists at Brookhaven use carbon dioxide $\left(\mathrm{CO}_{2}\right)$ as a probe for such energy-transfer collisions. Carbon dioxide is especially easy to detect because it can absorb or emit infrared light efficiently.

\section{The Bending and Stretching Molecule}

Carbon dioxide is a linear molecule made up of two oxygen atoms and one carbon atom, forming a line with the oxygens at each end and the carbon in the middle.
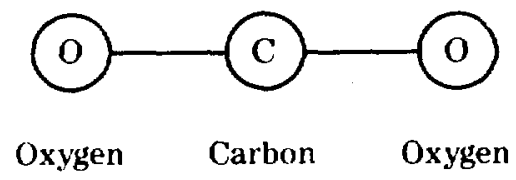

If a molecule in an excited state collides with $\mathrm{CO}_{2}$, it can transfer its vibrational energy to the $\mathrm{CO}_{2}$ and drop to a lower energy itself. Brookhaven chemists are investigating this, using azulene (a chemical closely related to the naphthalene used in mothballs), which is espe- cially easy to excite into a vibrational state with light from a laser.

Once the azulene has transferred some of its vibrational energy to the $\mathrm{CO}_{2}$, the excited $\mathrm{CO}_{2}$ molecule can undergo three types of motion: vibration (the molecule bends and stretches); translation (it travels steadily without spinning); or rotation (it spins). If the $\mathrm{CO}_{2}$ is vibrationally excited, it can bend and stretch in various ways.

The two oxygen atoms can move in the same direction, while the carbon moves in the opposite direction. This is called the antisymmetrical stretching mode:

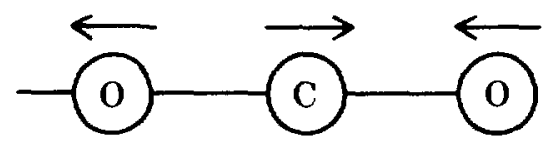

The oxygen atoms can move simultaneously in opposite directions while the carbon atom stands still, a process known as the symmetrical stretching mode:

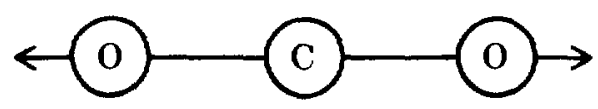

In the bending mode, both oxygens move in the same direction out of the line, while the carbon moves in the opposite direction:

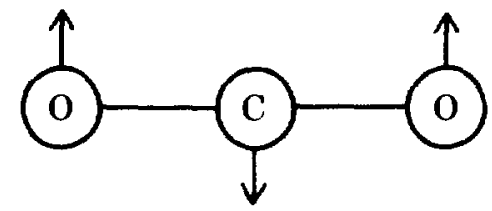

By looking at the absorption spectrum of $\mathrm{CO}_{2}$ in a diode-laser beam, Brookhaven chemists have determined the relative amounts of excitation in the symmetrical stretching, antisymmetrical stretching and bending modes.

Bending is favored. When the $\mathrm{CO}_{2}$ molecule "exercises" in this fashion, very little energy is put into symmetrical stretching, and it is 300 times more likely to bend than to stretch antisymmetrically. Also, 25 percent of all energy available to the $\mathrm{CO}_{2}$ molecule becomes vibrational, rather than changing into rotational or translational motion.

This new information, obtained through the extreme accuracy of modern tools, contradicts the previous, statistically based, general theory that energy is spread evenly among the different modes. No new theory of energy transfer has yet been formed to explain these recent observations.

\section{"Hot Atom" Energy Transfer}

Another form of energy transfer being studied at Brookhaven occurs when "hot" atoms hit a molecule. Unlike the straight energy transfer of the azulene and $\mathrm{CO}_{2}$ collisions, this can be a chemical reaction involving a chemical change in the collision partners.

"Hot" atoms are atoms that have much more energy than their surroundings at normal temperatures. Improved production techniques and methods of detection using lasers have encouraged research on what happens to this energy.

When hot hydrogen collides with carbon dioxide, its extra energy can excite the $\mathrm{CO}_{2}$ molecule in the same way that the vibrationally excited azulene can.

By looking at the infrared light emitted by $\mathrm{CO}_{2}$ in its excited state and also at how $\mathrm{CO}_{2}$ absorbs the infrared light of a diode laser, our researchers probe the various vibrational and rotational energy states. Once again, it is found that most of the vibrational energy appears in the bending mode of $\mathrm{CO}_{2}$.

The translational energy transfer is being examined with high resolution spectroscopy. This technique is especially useful in giving information on the velocity of a molecule since its spectrum changes according to its speed. On the spectrometer screen, a peaked-line profile indicates a slower-moving molecule; if the molecule moves faster, the line broadens. We found that the amount of energy going into the rebound of the hydrogen atom and the $\mathrm{CO}_{2}$ molecule is similar to the 
amount that one would expect in the collision of two microscopic billiard balls.

At certain energies, the hot hydrogen atom can react with $\mathrm{CO}_{2}$ and form carbon monoxide and an oxygen-hydrogen radical called hydroxyl.

$$
\mathrm{H}^{*}+\mathrm{CO}_{2} \rightarrow \mathrm{CO}+\mathrm{OH}
$$

A radical is a very reactive molecule with an unpaired electron that it seeks to pair up in a bond with another molecule. It is known from studies of combustion chemistry that this reaction involves the transient formation of the radical HOCO, which is stable at very low temperatures in condensed inert gases.

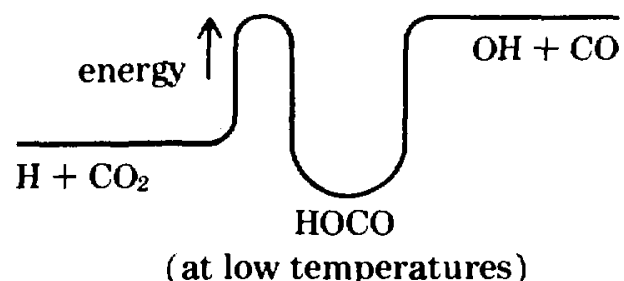

An important aspect of our study of the hydrogen and $\mathrm{CO}_{2}$ reaction is that it is the reverse of the reaction $\mathrm{OH}+\mathrm{CO}-\mathrm{H}+\mathrm{CO}_{2}$, known to be of special interest both in combustion and in atmospheric chemistry.

In combustion, it provides one of the best paths for the oxidation of carbon monoxide (CO), which allows a substance to burn more efficiently. In the upper atmosphere, hydroxyl radicals play a strong part in the removal of several gases that are involved in the "ozone hole" that has been a subject of concern recently.

\section{Exploding Molecthr Fuan Converuion}

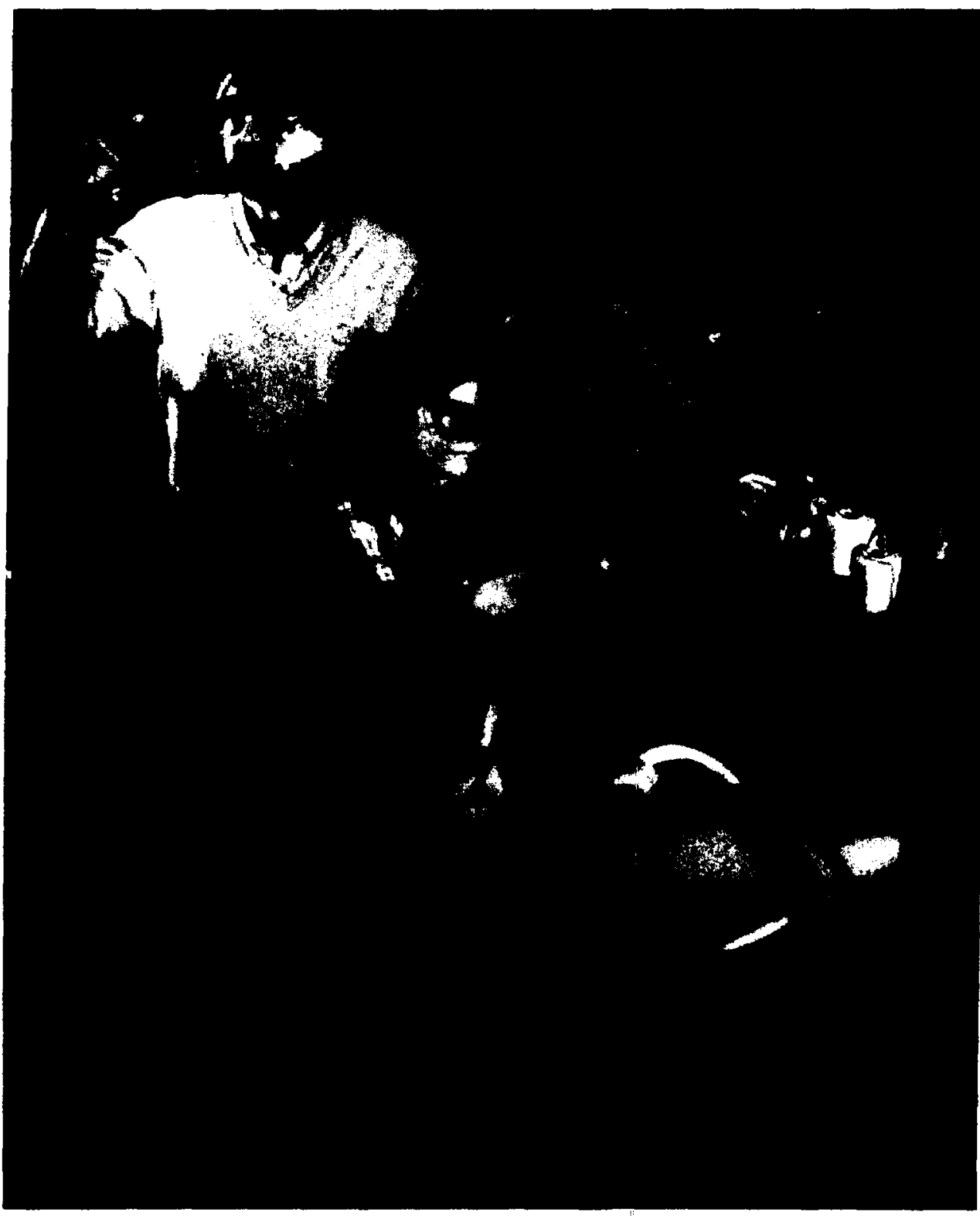

The shelves and benches of several laboratories in Brookhaven's Chemistry Department are filled with orange, red, blue, green and purple crystals and liquids. This rainbow of materials is being used to probe several fundamental aspects of energy conversion.

These materials contain metal complexes. A single metal complex consists of a metal core, such as an iron or cobalt ion, surrounded by ligands, which are molecules of, for example, water or ammonia. Metal complexes can be synthesized in the laboratory or can occur naturally. Examples of the latter are hemoglobin, which carries oxygen in blood, or cytochrome c, which transfers electrons in the mitochondria of plants and animals. Both contain an iron center, $\mathrm{Fe}^{2+}$, surrounded by a porphyrin ligand.

(From front) Brookhaven 's Etsuko Fujita and Carolyn Schurarz; David Szalda, a research collaborator from Baruch College; and Mei Chou, also of $B N L$, study the binding of carbon dioxide to a cobalt complex: They are urorking to characterize the "rays in which metal centers bind and activate carbon diocide toward reduction to potential fuels such as methanol or methane. 
The properties of metal complexes are determined by the nature of the central metal and the ligands surrounding it. By changing the metal, or the ligands, or both, the properties of a complex can be "tuned" for a specific purpose.

Brookhaven chemists use metal complexes to investigate basic processes related to the conversion of sunlight to fuels or electricity.

Several fundamental aspects of artificial photosynthesis are being studied. These include the capture of visible light, electron transport and fuel synthesis.

\section{The Capture of Visible Light}

The colors of the complexes are not just a delight to the eye, they are valuable in identifying the compounds. They provide information about the arrangement of the electrons around the metal center, and they are essential for the capture of visible light. Each color absorbs a different energy of light, so by changing the identity of the metal and the nature of the ligands, one changes the color and, therefore, adjusts the light energy absorbed.

The light absorption must also excite the metal complex for a relatively long time. In these conditions, the species "holds onto" the energy from the light so that it can be stored in later reactions.

The following diagrams show how the complexes can be changed to "tune" their light capture properties.<smiles>OP(O)(O)(O)O</smiles>

A metal center (an iron cation with a charge of plus two) surrounded by six water molecules is essentially colorless and poor for the capture of visible light. (In the diagram, the "O $\mathrm{O}$ " is nearer the "Fe," showing that it is the oxygen atoms in the water molecules that are bonded to the central iron.)

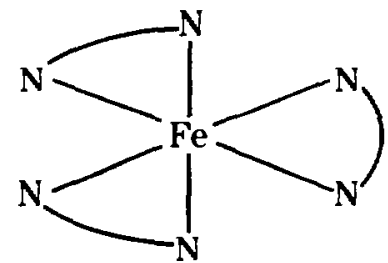

An iron plus two cation surrounded by three bipyridine molecules (attached to the center through their nitrogen atoms) is highly colored and absorbs visible light efficiently. But its excited state is too shortlived for the energy to be stored.

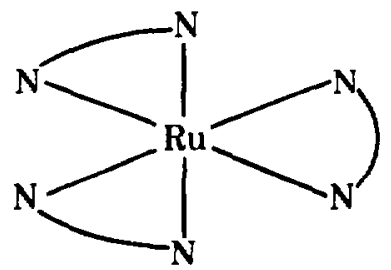

A ruthenium plus two cation surrounded by three bipyridine molecules absorbs visible light efficiently, $2+\quad$ has a long-lived excited state and transports electrons readily.

\section{Electron Transport}

Both natural and artificial photosynthetic processes depend upon efficient transport. In both, desirable electron transport processes compete with undesirable "back reactions" that re-form the starting materials and give off heat.

\section{Synthesis of Fuels}

In green plant photosynthesis, carbohydrates are synthesized from water and carbon dioxide. For artificial systems, Brookhaven chemists are exploring the transformation of water and carbon dioxide to oxygen and methane or methanol. Success could mean that sunlight and waste products may be harnessed commercially to produce fuel or electricity at minimum cost.

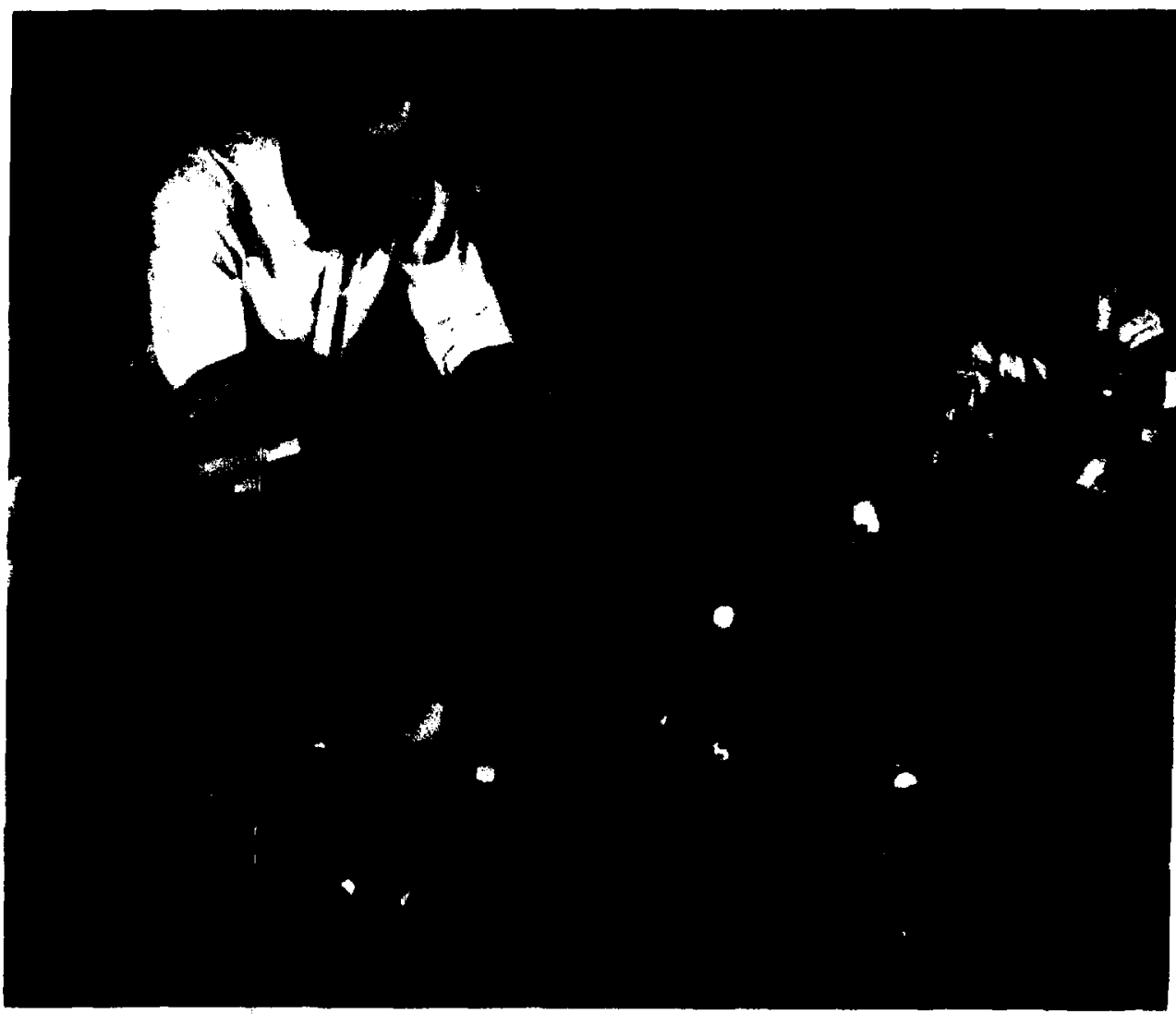

Jay Winkler (back, left), J-Jy Chang (front), Mariusz Kozik and other BNL chemists are exploring important factors that determine electron transfer rates: the separation between electron domor and receptor sites, the driving force for electron transfer, and the role of solvent dynamics. 


\section{The Great Solar Neutrino Trap}

Deep in a tunnel in the Abruzzi Mountains in Italy, an amazing feat of chemical detection is being arranged. In the Gran Sasso underground physics laboratory, well shielded from cosmic ray interference, researchers are setting up a 15,000-gallon tank containing 30 tons of the element gallium. In the tank, they hope to trap and detect solar neutrinos - ghost-like particles from the core of the sun.

The method being used was developed at Brookhaven in the late 1970 's by scientists from Brookhaven's Chemistry Department and the Max Planck Institute for Nuclear Physics in Heidelberg, West Germany. Now this original chemistry is to be carried out on the grand scale of the GALLEX collaboration.

GALLEX stands for "gallium experiment," and scientists from nine institutions in West Germany, Italy, France, Israel and the United States, as represented by Brookhaven, are participating in this cooperative research.

\section{An Interdisciplinary Experiment}

An unusual feature of the gallium experiment lies in its mixture of scientific disciplines. It makes use of nuclear chemistry, nuclear physics and chemical engineering, as well as

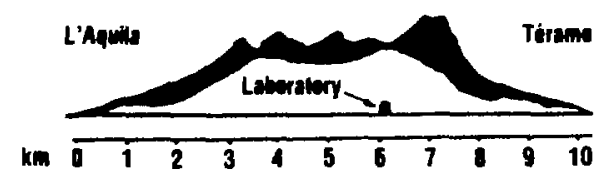

The GALLEX solar neutrino experiment is located in the Gran Sasso underground physics laboratory, deep in a tunnel in the Italian Abruzzi Mountains. civil engineering, needed for construction of the site and the apparatus.

The international scientific community's interest in solar neutrinos is twofold: New information will not only shed light on neutrinos, but also may reveal more about how energy is produced in the sun.

In standard theory, the sun's energy is thought to come from the hydrogen nucleus, which "burns" to form helium- 4 and other heavier nuclei. In several of these reactions, neutrinos are produced.

\section{Properties of Neutrinos}

Neutrinos are called "ghost-like" because they are electrically neutral and so small that, at rest, they may have zero mass. Also, they interact with matter so infrequently that they usually pass right through the Earth unnoticed.

Three kinds of neutrinos are believed to exist: the electronneutrino, which was discovered in 1953; the muon-neutrino, which was found in a Nobel-prize winning experiment at Brookhaven's Alternating Gradient Synchrotron in 1962; and the tau-neutrino, which has not yet been observed. They are partners of the electron, muon and tau particles, respectively, the members of the lepton family of particles, well-known in high energy physics.

The energy-producing reactions in the sun cause the emission of only electron-neutrinos. These reactions are thought to occur deep in the sun's core, which is extremely dense - 145 grams per cubic centimeter $\left(\mathrm{g} / \mathrm{cm}^{3}\right)$. Comparing this with the density of lead $\left(11.3 \mathrm{~g} / \mathrm{cm}^{3}\right), \mathrm{ol}^{\circ}$ water $\left(1 \mathrm{~g} / \mathrm{cm}^{3}\right)$, gives some idea of the density involved. Only neutrinos can pass through so much matter without getting stopped, so they are the only particles to escape.

\section{Capturing Neutrinos With Chlorine}

Although neutrinos are so elusive, they can be detected by sensitive nuclear chemical techniques. In 1967, in a deep salt mine in South Dakota, Brookhaven chemists started the first such radiochemical experiment to count solar neutrinos. As their neutrino trap, they used 600 tons of common drycleaning fluid, which contains carbon and chlorine. When a neutrino was captured, the chlorine- 37 in the liquid formed the isotope argon- 37 . This could be extracted from the liquid chemically and detected because it is radioactive.

A puzzling result was found. Scarcely one-third of the expected number of neutrinos were detected. This could mean that the standard solar model should be changed, or that neutrinos have characteristics not yet understood.

To confirm this result, another solar neutrino experiment was devised. Because most of the electron neutrinos from the sun have energies below the threshold for interaction with chlorine-37, another element, with a much lower energy threshold, was selected as the neutrino's target. This element is gallium.

\section{The Gallium Way}

The key to the gallium experiment is that the isotope gallium-71 becomes radioactive germanium-71 when it captures a neutrino. So, in the basement of Brookhaven's Chemistry Department, a great tank containing four tons of a solution of gallium chloride, water and hydrochloric acid was prepared for a large-scale test of the Brookhaven procedures. The isotope gallium-71 in the solution captured solar neutrinos, forming radioactive germanium-71. 


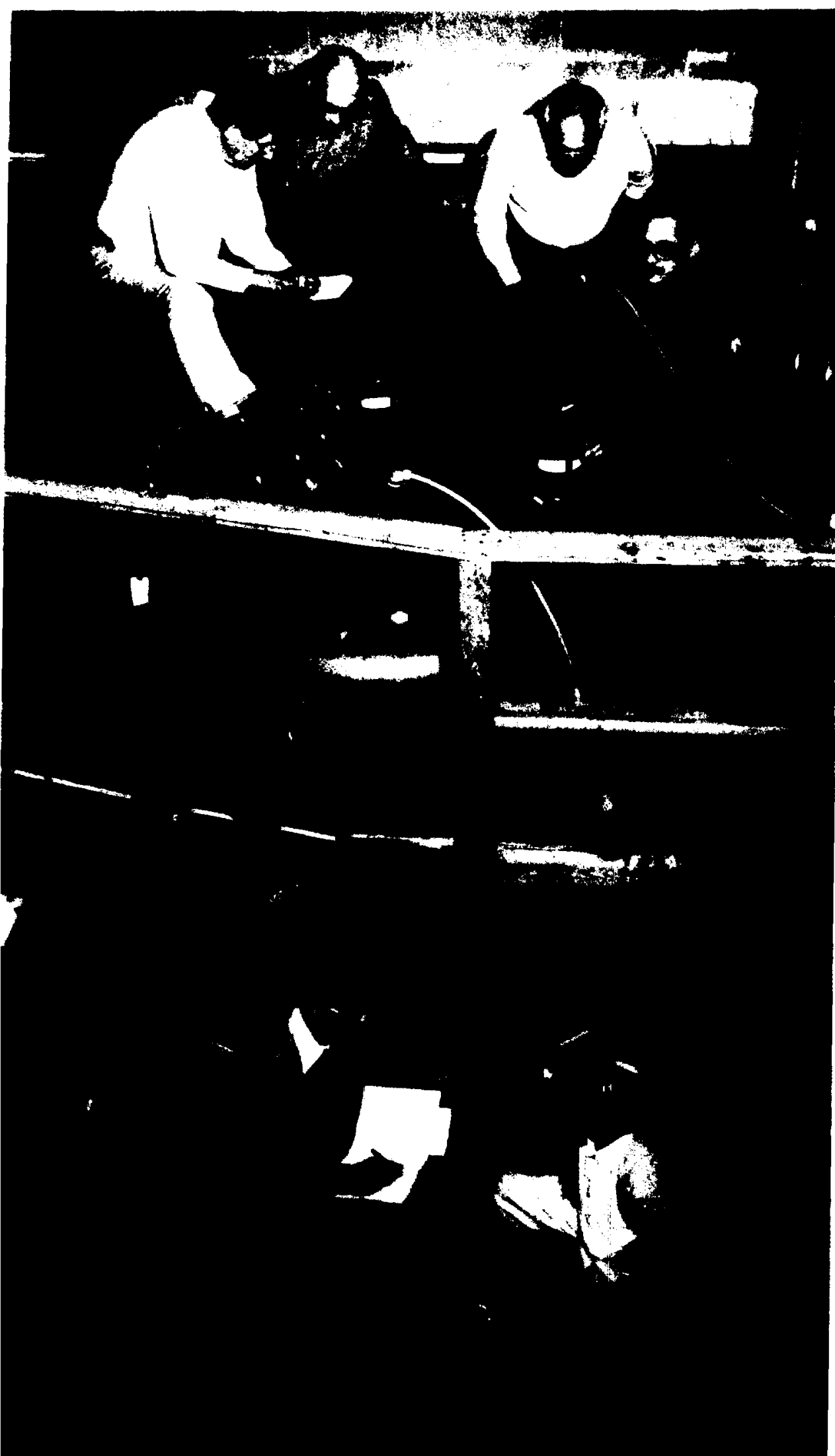

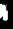

(Top, from left) John Galvin, Joseph Weneser, Gerhart Friedlander and Rirhard Hahn, and (bottom, from left) Keith Rowley and Dutch Stonener cherk the extrartion of germanium from the gallium chlorides solution in the Broskhaven pilot farility. The tank os be used in the GALLEX project is about 25 times the size of this ome.
In a complex combination of chemical techniques, it was shown that the germanium-71 formed a volatile chemical compound, germanium chloride, which could be swept out of the gallium solution with air. Next, the germanium-71 was trapped in water so it could be more easily purified. It was then converted to anot her gaseous form so that it could be introduced into a proportional counter, which detected the amount of germanium71 present, thus yielding the number of neut rino-incluced reactions.

\section{The GALLEX Results}

If the number of neutrinos detected conforms to the amount expected from theory, the result will mean that the "inner workings" of the sun and the properties of the neut rino are understood by scientists.

If, however, a result much lower than expected is found, it could mean that some of the electronneutrinos produced in the sun had changed into one of the other neutrino forms before reaching Earth. Such changes can only occur if the three neutrino forms have different, nonzero masses, a property of the neutrino that has been suspected, but never verified by experiment.

For the fields of elementary particle physics and astrophysics, therefore, the results of the GALLEX experiment should have profound implications. 


\section{Medical Department}

Scientists in the Medical Department use the unique physical and chemical science resources and other facilities at Brookhaven to develop new approaches to medical applications of nuclear technology and to understand human health effects of energy-related agents. Research issues include improved methods of radiotherapy and nuclear medicine procedures; development of new radiopharmaceuticals and methods for noninvasive measurement of human trace elements; and mechanisms of disease caused by energy-related agents.
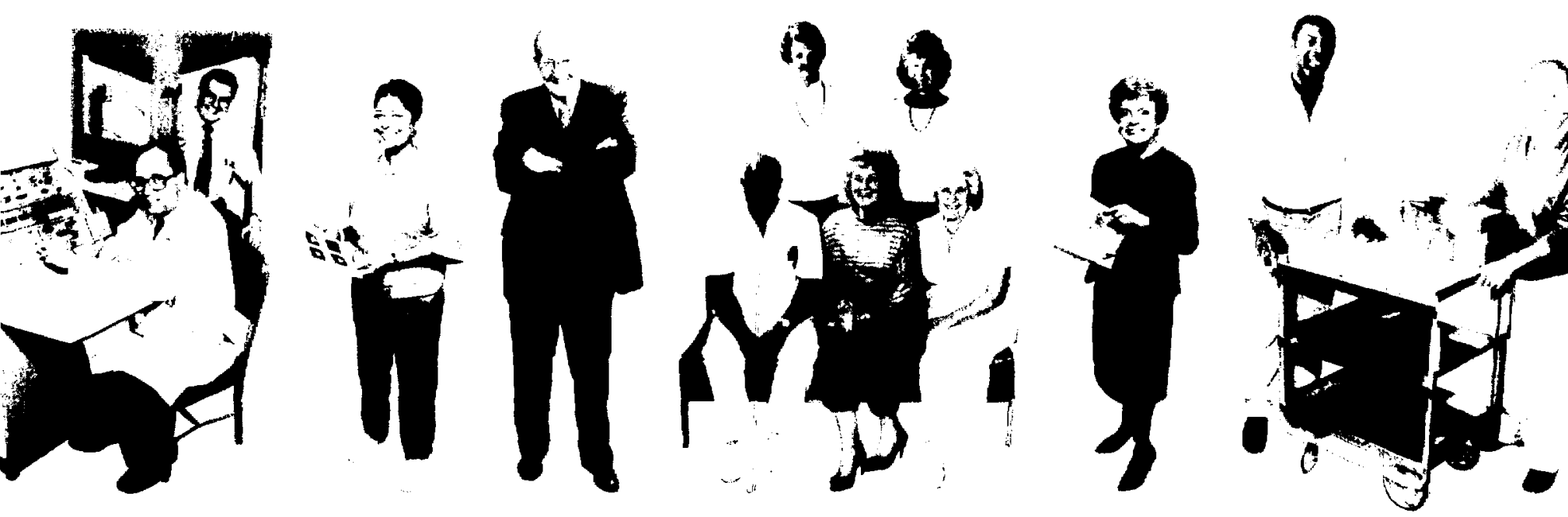


\section{Using PET to Study Cocaine Abuse And Brain Function}

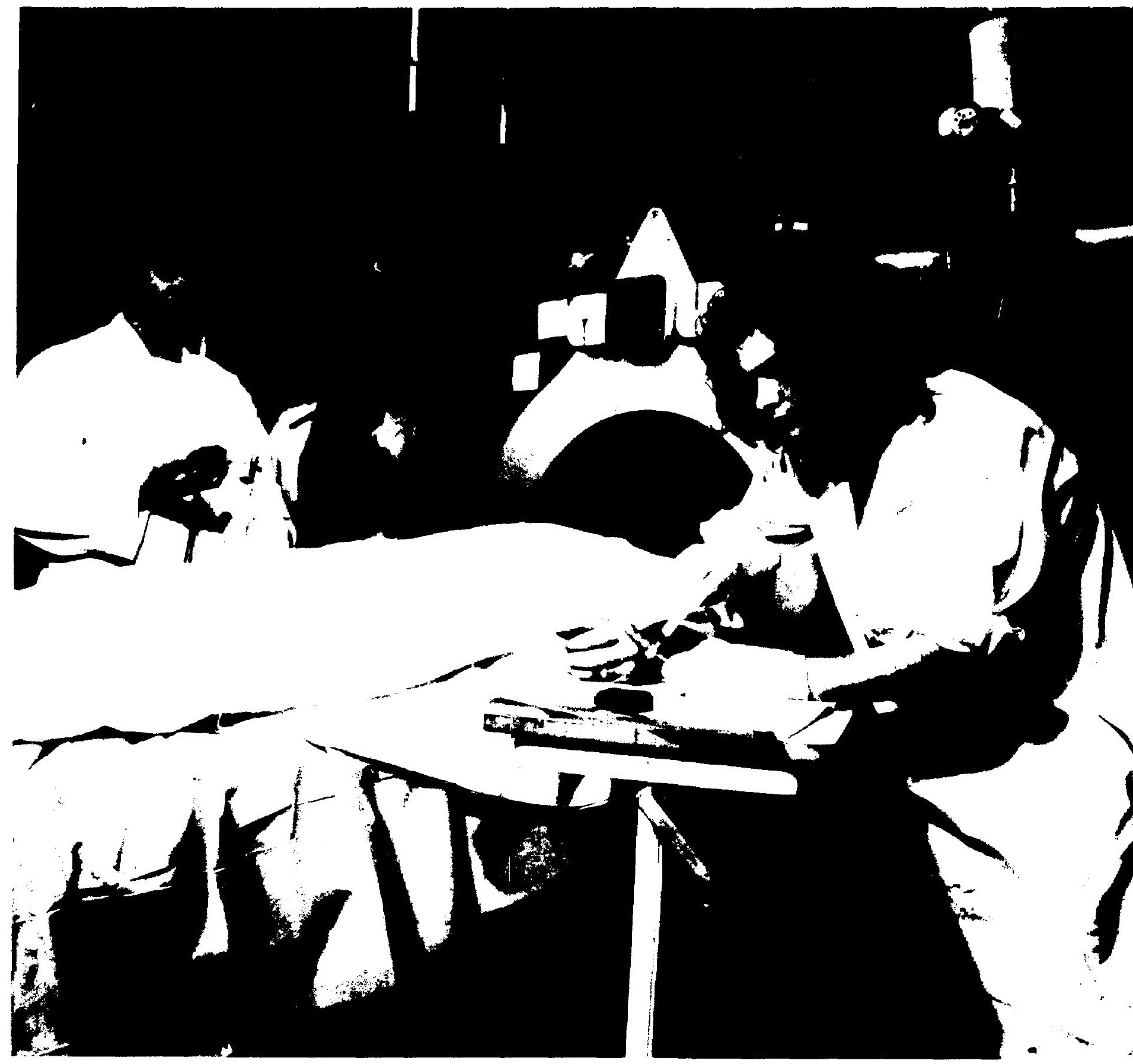

As part of a new study of brain neurochemistry as related to cocaine abuse, Nora Volkow (right) injects a patient with the radiotracer flworine-18fluonodeaxyghucose, as Noelwah Netusil (Reft) and Renee Moadel stand by with stopwatches to time the tracer's movement through the patient's bloodstream to his brain. The research is being dome at the PET facility, which is under the direction of Alfred Wolf. 
The numbers tell the story. In 1977 an estimated 1.6 million people in the United States used cocaine. By 1982, that number had risen to 4 million. Today, it exceeds 6 million - one out of every 40 Americans.

In collaboration with the Laboratory's Chemistry Department, researchers in our Medical Department have begun a new study of brain neurochemistry as related to cocaine abuse. Our main question is: What does cocaine do to the brain that makes the drug so addictive? And secondary to that, what is the extent of cocaine toxicity to the brain?

The work relies on a technique called positron emission tomography, or PET, which can be used to image areas of the brain. PET offers a first opportunity to look at human brain function.

Not so many years ago, cocaine was considered a benign drug, even by the medical community. Then, with pop heroes using it, cocaine became a fad drug, particularly in the form of crack, a cheap but powerful cocaine derivative.

High doses of cocaine can induce psychosis that most commonly shows as paranoia, but can manifest itself with visual and auditory hallucinations. The chronic cocaine user frequently becomes depressed, prone to impulsive suicide, and unable to concentrate, remember or think clearly. Cocaine abuse is not only a mental health problem, however. It is also a medical one. The drug is highly toxic and can damage the brain, heart, lungs and eyes. It can lead to serious obstetrical complications and, in newborns, problems of the vascular system.

of the habit-forming drugs, cocaine and heroin are now con sidered the most powerful reinforcers, as evidenced by how quickly animals acquire a behavior of selfadministration. Animal studies have shown that, when given free access to cocaine, animals will choose it over food and sex.
In people, little is known about the effects of cocaine on the brain's neurotransmitters, which are substances that carry nerve impulses from one neuron to another across a junction, or synapse. Preliminary research on humans shows similarities to animal studies, where it has been found that acute cocaine usage blocks the reuptake of dopamine, a neurotransmitter that plays a role in the reward centers of the brain. By stimulating the dopamine centers directly, cocaine activates the reward centers of the brain, without the animal actually carrying out behaviors that lead to pleasure.

Within this framework, one of our working hypotheses is that the brain's dopamine systems play a role in regulating mechanisms of reward and dependence for cocaine.

In our first study of a small group of chronic cocaine abusers, the procedure was to monitor the brain's dopamine activity with fluorine-18 n-methylspiroperidol $\left({ }^{18}\right.$ FNMSP). The distribution of ${ }^{18}$ FNMSP in the brain is monitored with the PET machine, and these data are then transformed by means of a mathematical model to obtain information about the dopamine receptors.

Our preliminary results show decreased binding of ${ }^{18}$ FNMSP in the cocaine abuser, suggesting a decrease in the number of dopamine receptors in these patients, possibly as an adaptation to overcome the increases in dopamine due to cocaine. In addition, although dopamine activity does increase immediately after cocaine is taken, chronic cocaine use may lead to an actual decrease in dopamine activity in the brain because of depletion from overstimulation. These changes are important inasmuch as they could explain the drug craving observed in these patients upon cocaine withdrawal.

To address the question of cocaine toxicity, we have done studies with fluorine-18 fluorodeoxy- glucose (FDG). This short-lived radioactive tracer behaves essentially the same as glucose, which the brain uses for energy. Glucose uptake reflects brain activity. Brain scans of chronic cocaine abusers show widespread areas of low FDG uptake, an indication of brain damage.

We have also looked at the effects of cocaine on cerebral blood flow. What we have found are areas of deficient blood circulation, similar to the lesions seen in brain scans of people who have had small strokes of the cortex. We hypothesize that cocaine abuse may destroy brain tissue because of its direct effect on blood vessels, where it induces vasoconstriction, which in turn can lead to tissue destruction.

Our aim is to re-examine patients three months after they are off the drug, so we can assess the reversibility of brain damage. It is also a way to evaluate treatment and learn in general what to expect in recovery.

In the future, we plan to monitor directly the distribution of cocaine in the brain using cocaine labelled with a positron emitter. This strategy will enable us to localize the areas of the brain affected by cocaine. We also hope to apply the PET findings to the design of therapeutical trials. For example, if we confirm that chronic cocaine use causes dopamine deficiency, that would support the treatment of cocaine craving and withdrawal with drugs that enhance dopamine activity in the brain. 


\section{Hope for Incurable Cancers}

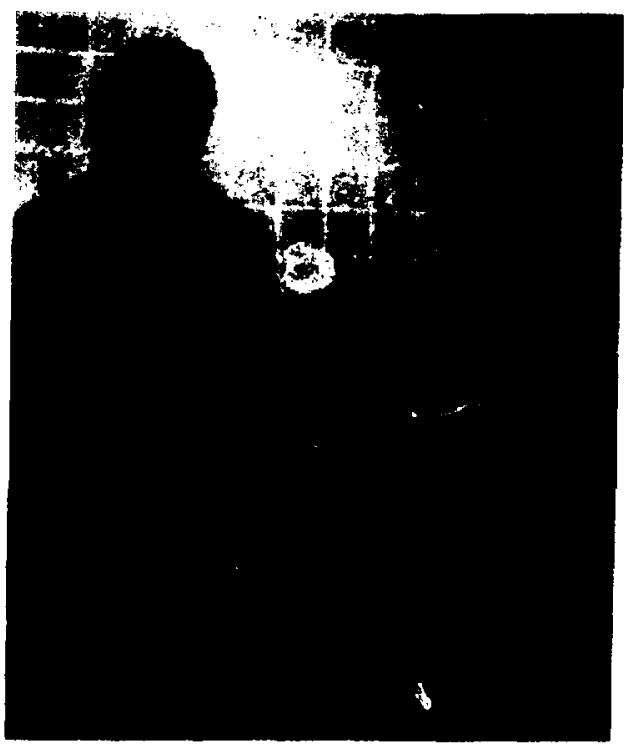

In collaboration with researchers in BNL's Medical Department, Joseph Goodman (pictured), a neurosurgeon at Ohio State University Hospital, and Reinhard Gahbauer, head of radiation oncology at the hospital, conduct clinical trials to test photon activation therapy on a type of brain tumor. Mounted on the plastic head is an apparatus used to implant a tiny radioactive source into a tumor. Photo courtesy of Ohio State University Hospital.
A new technique developed at Brookhaven promises to make some presently incurable cancers more susceptible to radiation therapy. Clinical trials conducted by research collaborators at Ohio State University Hospital are already under way to test the technique on glioblastoma, a lethal type of brain tumor.

The technique is called PAT, for photon activation therapy. Critical to its success are two components: iodinated deoxyuridine (IdUrd), which is a radiation sensitizer, and samarium-145, a radiation source.

For reasons that are not yet clearly known, iodine enhances the sensitivity of cells to radiation. IdUrd, an iodine-based drug, is a vehicle for incorporating iodine into cells. This is because IdUrd is structurally similar to thymidine, which is found in the cell nucleus.

When cells divide, they synthesize DNA, a process that ordinarily uses thymidine. If IdUrd is present, some of it is incorporated into the DNA in place of thymidine. The theory behind photon activation therapy is to enhance the radiation dose to the tumor by introducing iodine into tumor cells, via IdUrd. Because tumor cells proliferate rapidly, they will incorporate significant amounts of IdUrd. (Since the brain does not grow new cells, normal brain tissue will not take up IdUrd.)

Tumor cells, however, are not the only ones that will readily take up IdUrd - so will bone marrow, gut and skin cells, all of which can divide about as rapidly as tumor cells. How, then, can normal tissue cells be protected from radiation?

The answer lies in taking a source of radiation - in this case, a sample of samarium-145, about the size of a caraway seed - and implanting it directly in the tumor. Samarium145 gives off low energy photons.
Thus, because the intensity of radiation falls off quickly with distance, only the sensitized, nearby cancer cells are killed.

Another important aspect of photon activation therapy is that when the iodine is irradiated with the proper energy photons, it becomes activated and unleashes a cascade of electrons that are at least as damaging to the cancer cells as is the original photon radiation.

This electron shower is known as an Auger (pronounced o-zhay) cascade. The idea of using Auger cascades for cancer treatment is not new. Initially, radioactive iodine-125 was tried as the cascade source. Iodine-125, however, is taken up by other rapidly growing normal cells (such as bone marrow and gut) and can deliver toxic levels of radiation to these sensitive normal tissues before a tumor-lethal dose is achieved.

The Brookhaven technique, instead, uses IdUrd with nonradioactive iodine- 127 as the radiation-sensitizing agent and electron-cascade source. Thus, normal tissue is excluded from the treatment.

As part of phase one of the clinical trials in Ohio, researchers are studying the distribution of IdUrd in glioblastoma, as well as the general effect of the drug on patients. Although at present the IdUrd is being activated with implanted sources of high energy iridium-192, the intent is to switch to samarium145 when it becomes commercially available. An additional advantage of the samarium source is its long shelf life of one year, as compared to the 60-day shelf life of iodine-125 and the comparably short shelf life of iridium-192.

Although we are years away from knowing the outcome of these clinical studies, Brookhaven's photon activation technique brightens the prognosis for dealing with incurable cancers. 
change, cancer or the cell's death. The probability that the energy deposited in a cell will severely damage the cell in this way can be assessed using what is called the hit-size effectiveness function (HSEF), a true dose-response function for the cell that has an energy threshold.

Given that cancer starts with damage at the single cell level, a more precise way to do risk assessment may be to rely not on predictions based on cell response as a function of organ dose, as is done at present, but on predictions based on cell response as a function of cell dose, i.e., on the concept of HSEF. Risk assessment done this way would be generally applicable to any radiation field, of either single or mixed quality.

An HSEF has been evaluated accurately, using cells of the plant Tradescantia. What is required next is to establish an HSEF for human cells, as well as for cells of other plant and animal systems.

In 1988 , we began a project on HSEF for human chromosome aberrations. The procedure involves tak-

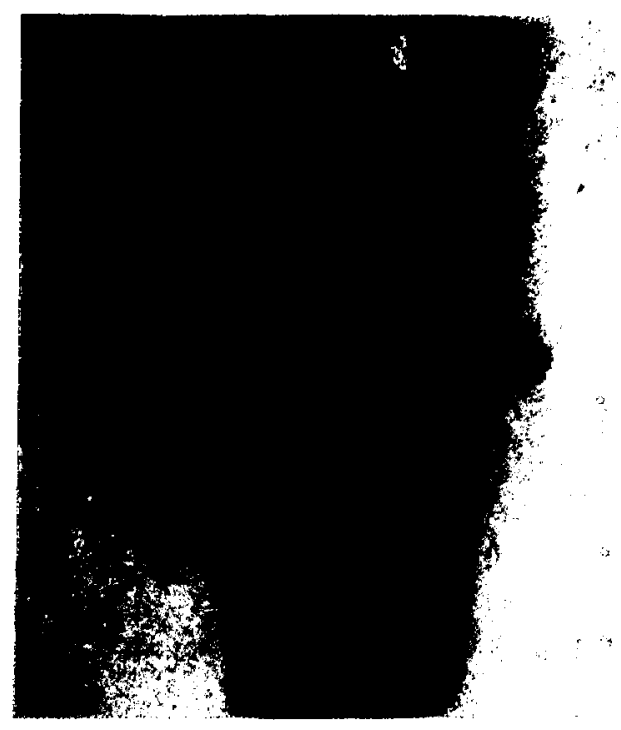

In this picture of human chromosomes, the markers indicate chromosome translocations, a class of aberration implicated in many human cancers.

ing chromosomes from normal human blood and irradiating them with neutrons of different energies to produce abnormalities. The chromosomes will then be examined for transformations with a cytoscanner, a machine that can score a large number of chromosomes quickly. Finally, sophisticated mathematical and computer techniques will be applied to derive an HSEF.

While damage occurs all the time at the cellular level, cells also constantly repair themselves. The difficulty in evaluating low-level exposure is that only a small fraction of those cells exposed will be damaged. Further, it's not enough to be hit. A cell must be hit hard for a severe and permanent change to occur. Thus, we will look only at breaks in the chromosomes and at translocations (the permanent transfer of a chromosome segment from its usual position to a new position on the same or different chromosome).

Critical to this project is proving that the cell response is a function of the energy deposited there. We have to be sure that we are measuring only that part of the radiation field that the biology is seeing.

It will take about three years to collect enough data. By then, we hope to be able to show that HSEF is a better approach for evaluating radon exposure, as well as low-level exposure in general. 


\section{Biology Department}

Research in the Biology Department encompasses studies on molecular structure, molecular genetics, DNA damage and repair, and cell biology of plant and animal systems. The department contains the Scanning Transmission Electron Microscope Facility and also operates stations for molecular structural studies at the National Synchrotron Light Source and the High Flux Beam Reactor.
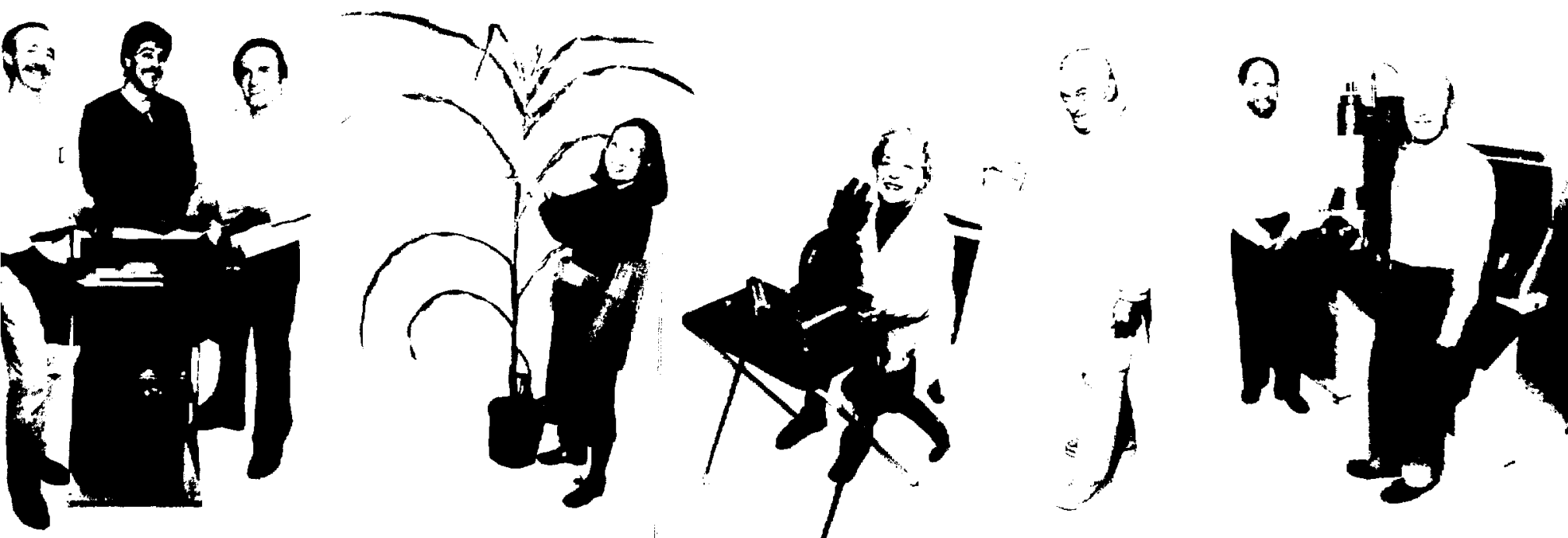


\section{The Enigmatic Enzymes}

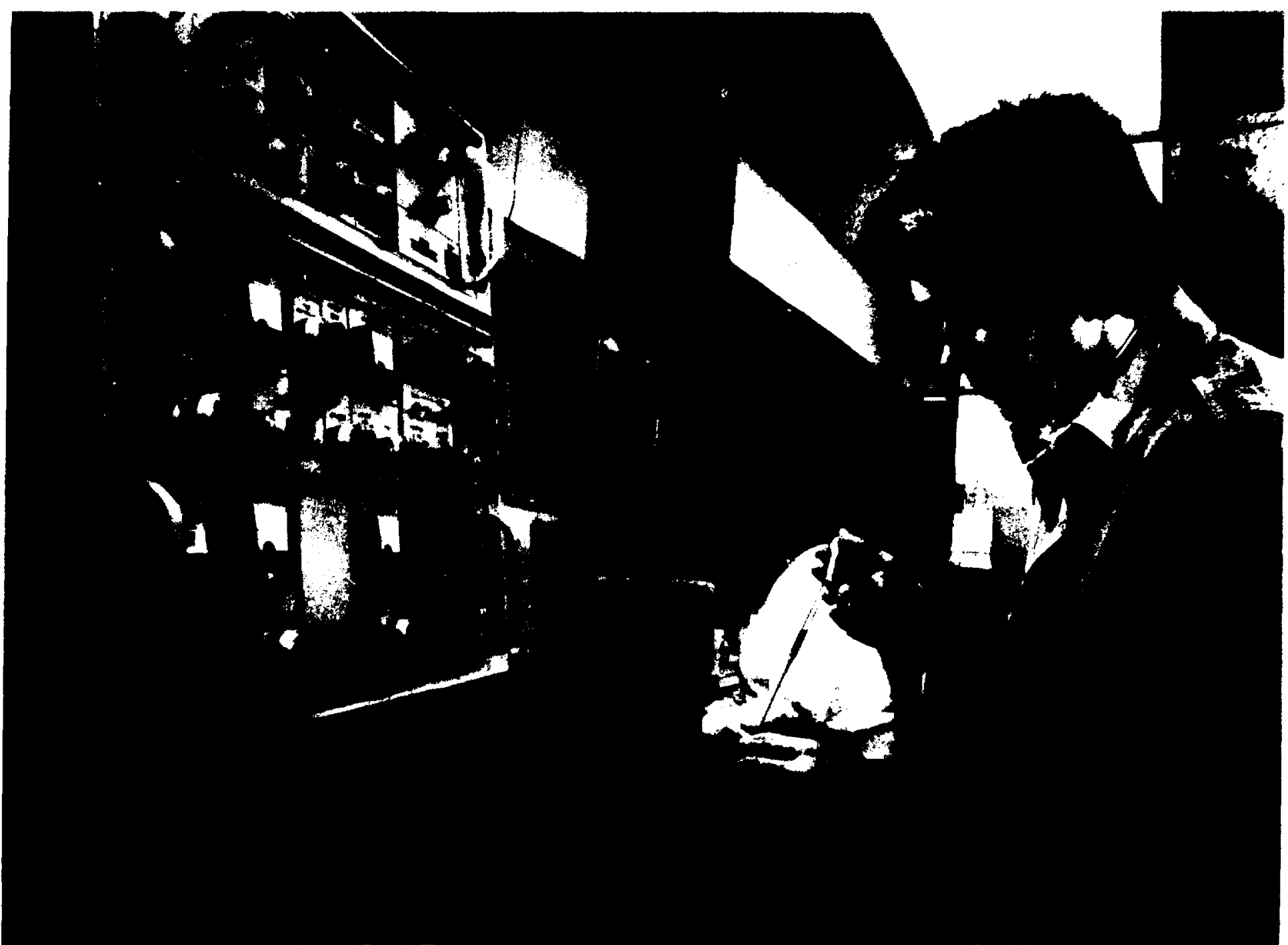

Walter Mangel is adding a fluoroge:'ic substrate to test tissue plasminogen activator, while Liana Toleds prepares the computer to record the data.
A cancer cell penetrates a blood vessel.

A blood clot dissolves.

An egg bursts out of the ovary.

Common to these processes is the breakage of certain biological structures at specific times and places. During ovulation an egg breaks through the wall of the ovary. A heart attack stops when the blood clot causing it is broken up. Metastasis occurs when cells from a malignant tumor break into a blood ressel and travel throughout the body to establish secondary tumors.

Bislogists have discovered that these and other apparently umelated physiological processes may involve the same enzyme system in the body.

Using Brookhaven's unique facilities, scientists in the Biology

Jepartment are studying this enzyme system, how it works and how it is used in so many different ways.

Enzymes are a class of proteins that accelerate the rate of a biochemical reaction. The particular enzymes incolved in the physiological processes described here are called the proteinases of the fibrinolytic sisstem. I'nder different conditions, they can produce different efferels. some normal and useful. some ditngerous to headth. 
Brookhaven biologists are investigating how these enzymes function. As various aspects are understood, the expectation is that these enzymes can be manipulated so that only their beneficial aspects are used.

The starting point in this fibrinolytic enzyme system is the protein plasminogen. It is present in all body fluids and is an inactive enzyme. Plasminogen can be transformed by another proteinase called plasminogen activator into an active proteinase, plasmin. Plasmin is the highly destructive enzyme that, for example, destroys the protein fibrin, the major component of blood clots.

For at least ten years, it has been known that the immediate cause of a heart attack is a fibrin blood clot that blocks the flow of blood to the heart. It has also been recognized that a normal function of fibrinolytic enzymes is to activate plasminogen at the site of a clot so that the resultant plasmin will destroy the clot.

Recently, advanced techniques in genetic engineering have made it possible to copy, or clone, plasminogen activator so that large quantities of it are available to treat heart attack patients. The new drug, called tissue plasminogen activator, is now approved by the U.S. Food and Drug Administration. It dissolves blood clots in over 60 percent of the patients experiencing a heart attack.

While these clinical benefits are obvious, however, exactly how this enzyme system functions is not yet known. Plasminogen and plasminogen activator are present continuously in all body fluids - yet they do not interact. What causes them to interact at the site of a fibrin blood clot? When an egg is ready to leave the ovary, what signals it to

In this tumor cell, the brighter areas of white show the location of plasminoyen activator at urork on the cell surface. secrete large amounts of plasminogen activator? And during metastasis, how can a cancer cell damage its environment to penetrate a blood vessel, yet protect itself from harm?

The basic question is: What are the biochemical mechanisms that trigger the activation of plasminogen at specific times and places?

Biologists believe the answer to be related to the observation that both plasminogen and plasminogen activator bind to the objects that are targeted for destruction. Once bound to a target, these two enzymes change their shapes and, as a result, become much more reactive in producing plasmin.

Brookhaven's facilities enable us to study these changes in plasminogen's shape and structure. At the High Flux Beam Reactor, a beam of neutrons is aimed at plasminogen, and, by the way the neutrons are scattered, the shape of plasminogen can be deduced. At the National Synchrotron Light Source, a beam of ultraviolet light is absorbed by plasminogen. The way the light is absorbed is indicative of the structure of plasminogen.

One finding is that when plasminogen binds to its target (for example, fibrin), the largest structural change ever seen in a protein occurs. This change makes the bound plasminogen much more reactive than unbound plasminogen.

In the course of our research, we have designed and synthesized new organic compounds, which have been patented. These are used in the laboratory to detect fibrinolytic activity. Some of them will soon be used in clinical laboratories to determine whether certain blood and urine enzymes are present at normal levels.

These compounds are also being used with breast cancer patients to indicate whether specific tumors will respond to hormone therapy. Although tissue plasminogen activator is an effective drug in the treatment of heart attack, the search is on for a "second generation" drug that is even more effective, and these compounds are being used in some of the screening procedures.

The more we learn about the fibrinolytic enzyme system, the greater the potential for medical applications of that knowledge. The first new heart attack drug in many years is now in use - and it's a success. It may be the harbinger of other benefits to be derived from studying the fibrinolytic enzymes.

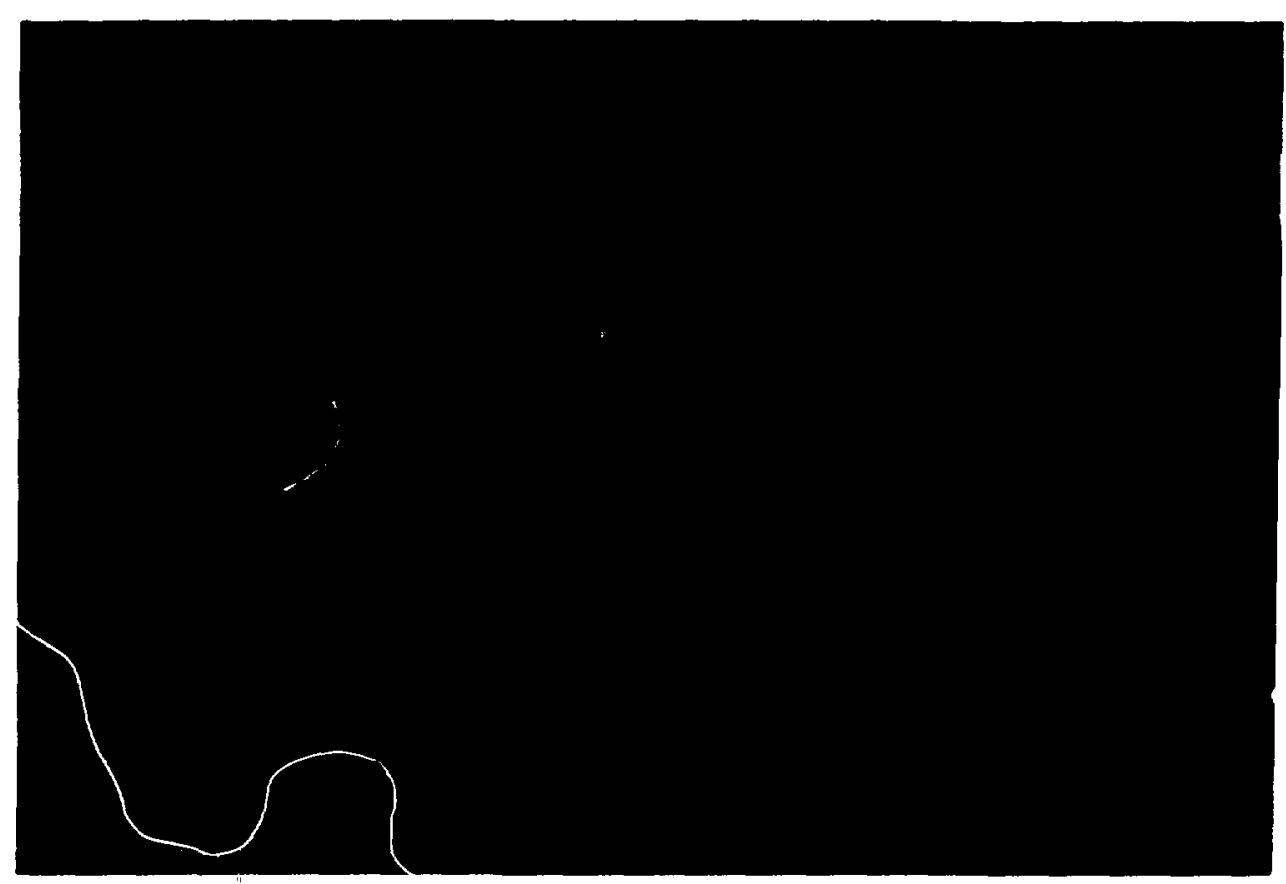




\section{Unraveling the 30-nm Filament}

\section{What's a 30-nm Filament? Read This First!}

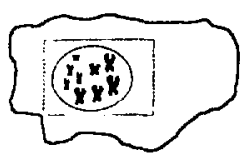

CELL - Cells are the smallest autonomous part of a living organism. Cells of higher organisms, such as humans, originate from a single cell, which, as it divides, develops into specialized forms such as various olgans.

NUCLEUS - Nearly every cell from a higher organism has a nucleus, which contains the genetic material of the cell.

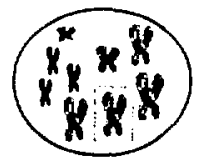

DNA - DNA, short for deoxyribonu-

cleic acid, is a long molecule
containing the genetic information that the cell needs to function and multiply. It consists of two strands wrapped around each other.

GENE - A portion of DNA containing information that can be translated into a protein or some other functional molecule.

CHROMOSOMES - These units contain the genetic material of cells. Each chromosome carries many genes and consists of proteins bound to DNA. Just

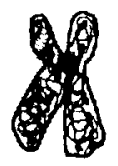
before and during cell division, chromosomes become compact and assume characteristic shapes; they are then visible as separate entities.

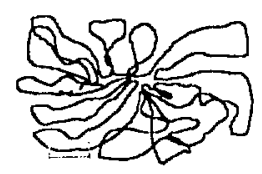

CHROMATIN - Between cell divisions the chromosomes of cells are partially unraveled. This form is referred to as chromatin.

30-nm FILAMENT - When chromatin is examined under an electron microscope, one sees that it is

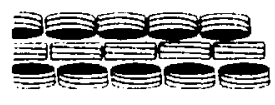
made up of fibers that are about 30 nanometers $(\mathrm{nm})$ thick. These fibers are called $30-\mathrm{nm}$ filaments.

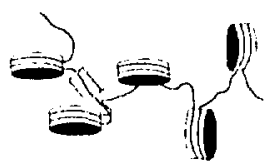

NUCLEOSOMES and LINKER DNA If the structure of the $30-\mathrm{nm}$ filament is disrupted by placing chromatin in a solution that has

very low salt, chromatin takes on the appearance of beads on a string. This form of chromatin is called the $10-\mathrm{nm}$ filament. The beads are called nucleosomes, and the string is DNA. The part of the string between nucleosomes is called linker DNA.

Each nucleosome consists of a protein core around which two turns of DNA are wrapped.

HISTONES - These proteins bind to DNA to form chromatin. Four of these form the core of nucleosomes, and a fifth type binds to the outside of nucleosomes and to linker DNA.
$\mathrm{T}$

Think of string packed into the inside of a ball. Now picture the string as 300,000 times longer than the diameter of the ball. And, to make it more complicated, different parts of the string must be easily accessed from the outside without unraveling the rest. This image is akin to the 1.8 meters of DNA packed into a cell's nucleus, which is only six millionths of a meter in diameter.

As an organism develops from a single cell, this DNA is reproduced so that each specialized cell (for example, a skin cell or a white blood cell) receives a copy of the DNA. These different types of cells, however, use different genes of the DNA. Each cell functions by the action of proteins on these regions of DNA. So, although so much DNA is packaged into such a small space, these regions must remain easily accessible, or the proteins cannot get to them. Understanding how this is done is a major problem facing molecular biologist today.

In Brookhaven's Biology Department, we study the structure of one tiny part of this amazingly organized DNA packaging - the 30-nanometer (nm) filaments. These filaments are visible as threads that make up chromatin.

When the cell is not dividing or preparing to divide, the DNA is organized as chromatin. There is evidence that the structure of chromatin determines whether the genes on it are accessible. Once this basic structure is understood, it will help us see how the various proteins modulate that structure and, thus, learn more about how genes work.

There are at least four different models of how the chromatin is organized to form 30-nm filaments. The solenoidal model proposes that neighboring nucleosomes along the DNA are in contact. In other models, they lie either across from or above one another in the 30-nm filament.

To find out which of the models is correct, our researchers are performing detailed experiments using two entirely different techniques.

In Brookhaven's Scanning Transmission Electron Microscope (STEM), we observe individual molecules of chromatin directly and measure their mass and size. At the High Flux Beam Reactor (IHFBR), we 
bombard the chromatin with a beam of neutrons. The pattern in which the neutrons scatter after hitting the chromatin sample yields information about its structure.

Brookhaven's advantage lies in having both STEM and the HFBR available close to each other, so that these complementary techniques can be applied to the same problem. In fact, experimental results from the STEM and HFBR facilities have proved to be in good agreement on the same sample, no differences arise from the investigative technique.

Our studies show that the values for the mass of the filament are consistent with those of the solenoidal model, which are much smaller than the masses predicted by some of the other models.

While these initial experiments give us some idea of the structure of the 30 -nm filament, much more information can be obtained by locating individual components of the structure. We have concentrated on locating a particular protein, histone $\mathrm{H} 5$, which is essential to organizing chromatin in the $30-\mathrm{nm}$ filament. The several chromatin models predict different locations for $\mathrm{H} 5$.

Recently, we showed that it is possible to remove $\mathrm{H} 5$ from the filament, replace it, and still regain the original neutron scattering pattern. This experiment also showed that about six consecutive nucleosomes must bind $\mathrm{H} 5$ in order to form the 30-nm filament.

To locate H5, we plan to put a deuterated version of it ( $\mathrm{H} 5$ in which the hydrogens are replaced by deuterium) on the chromatin. Deuterated proteins scatter neutrons differently, so the new scattering patterns will show the deuterated $\mathrm{H} 5$ 's position.

One problem is that higher organisms such as chicken (the source of the chromatin we study) cannot be deuterated. Since the bacterium $E$. coli produces deuterated protein when grown in heavy water, we

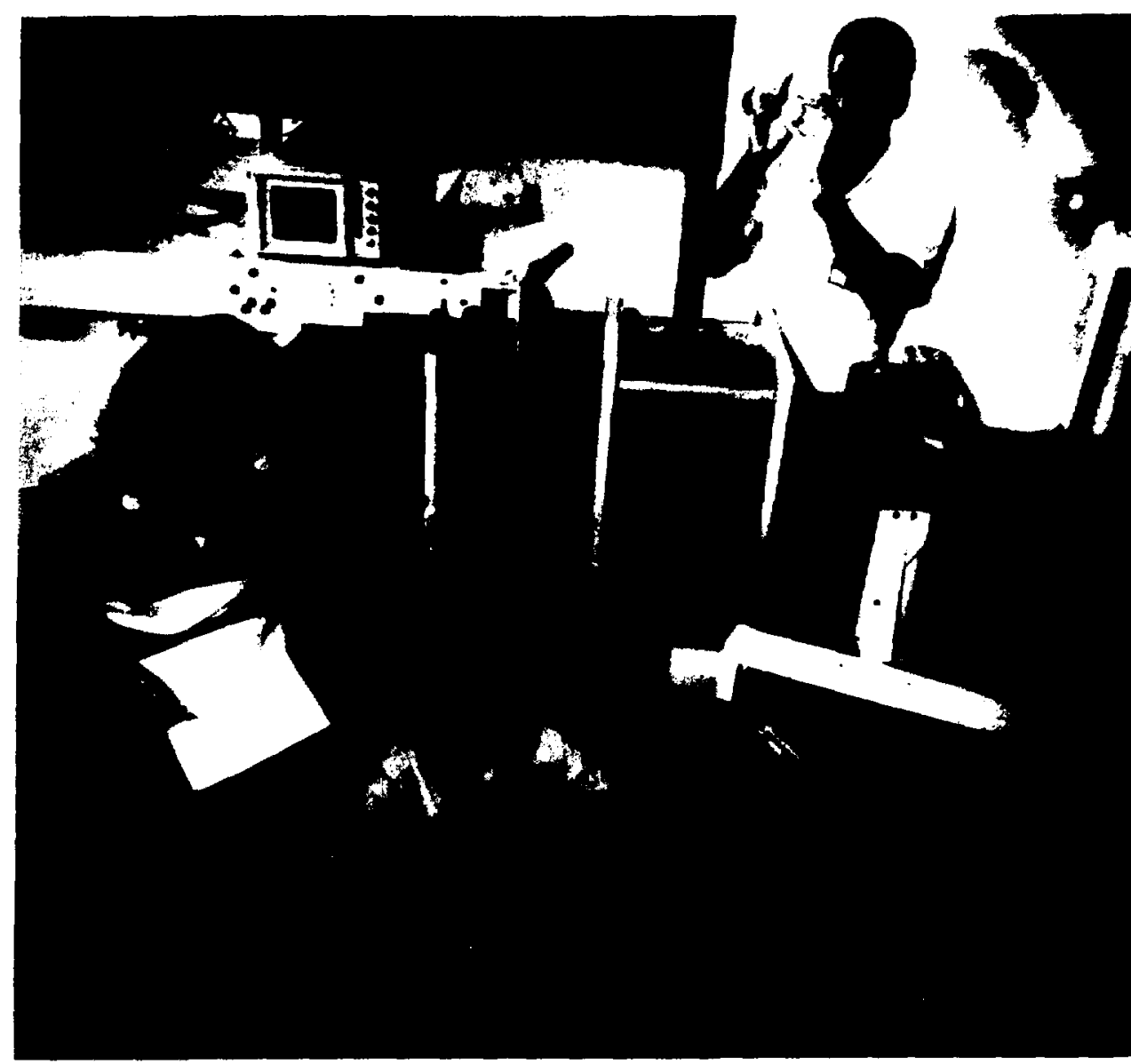

At the High Flux Beam Reactor, Venkatraman Ramakrishnan (top) examines a chromatin sample contained in a quartz cell, while Sue Ellen Gerchman and Vito Graziano check the temperature of the sample changer.

have taken the gene for histone $\mathrm{H5}$ from chicken and are trying to trick $E$. coli into making it.

In the end, our detailed work is expected to shed light on the structure of the 30-nm filament, the pre- dominant form of chromatin in the cell. On this new information, other research will be built, leading to an understanding of how the cell organizes its genetic material.

\section{Hatchet Men of the Pneumonia Bacteria}

M afraid of catching a virus, drink more orange juice, avoid crowds and hope for the best. Bacteria, however, have a much more effective defense system. They have at their disposal special "hatchet men," restriction enzymes that attack viruses and chop them into small, harmless pieces.

\section{$D p n I$ and $D p n I I$ are restriction} enzymes in the bacterium Streptococcus pneumoniae. They are the endonuclease components of restriction systems, which recognize and cleave into pieces the DNA of many viruses that threaten the bacterium. Discovered by scientists in Brookhaven's Biology Department in the mid-1970's, the $D p n I$ and $D p m I I$ 


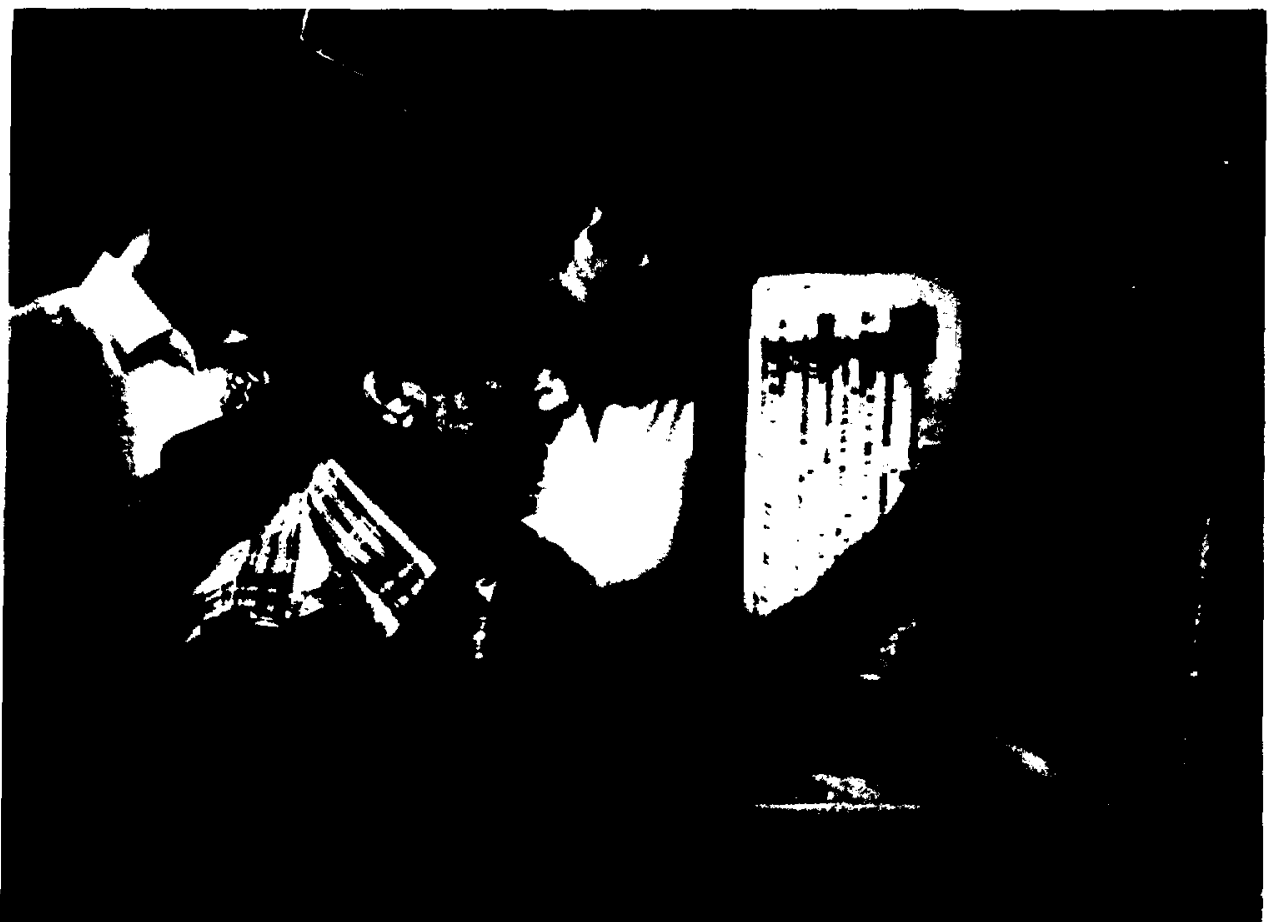

From left, Bill Greenberg, Sanford Lacks and Sylvia Springhorn examine autoradiograms that reveal the DNA sequence of genes encoding the restriction enzymes of Streptococcus pneumoniae.

restriction enzyme systems provide a valuable subject for research in the fields of genetic regulation and engineering.

Enzymes are proteins that are catalysts for the chemical reactions constantly taking place within all organisms. Restriction enzymes do what their name suggests: they restrict something, in this case infection by a virus. They do this in a very active way, by cutting up the genes of the virus before the virus can take control of the bacterium.

A virus particle often has a nucleus of double-stranded DNA containing the genetic information that enables the tirus to infect a cell. The DNA is formed of various combinations of four nucleotide bases: guanine $(G)$, adenine $(A)$. thymine ( $\mathrm{T}$ ) and cytosine $(\mathrm{C})$. By the order in which the nucleotide sequences are arranged and repeated, the DNA contains information recognizable as specific genes.

Genes command the characteristics of an organism. The geness themselves are linked together to form chromosomes. Each particular organism has a specific number of chromosomes that characterize its entirety.

When a virus injects its DNA into a bacterium, the bacterium's restriction enzymes recognize a certain pattern of the INA in the virus. Wherever that pattern occurs, the restriction endonuclease will cut the DNA - perhaps 100 times in each viral INA. The virus is then unable to reproduce and becomes harmless. This can reduce the rate of infection by a millionfold.

The ability of restriction enzymes to recognize and cut DNA at specific points has another advantage. Scientists use these enzymes to cut out and then copy, or clone, specific pieces of DNA, thus furthering genetic engineering research.

The two restriction enzymes discovered at Brookhaven in different strains of $S$ premomoliae behave in an especially interesting way. They contain methylases that modify INA. The first to bo found was $I P m I$. and it cuts up viral INA where the nucleotides are arranged as "(ATC:" But I pma only cleaves this INA if the $A$. of adenine, in the sequence is modified by having a methyl group attached to it.

As the adenine in the DNA of $S$. pneumoniae containing $D p n I$ has no methyl group attached, the $D p n I$ will not cut up the DNA in its own cell.

If $D p n I$ is not at work, the second $S$. pneumoniae restriction enzyme found at Brookhaven, DpnII, takes its place. DpnII also cuts DNA at GATC - but only if the adenine in that sequence is not methylated. This means that if the DNA in DpnIrs own cell were unmethylated at adenine in GATC, it would be destroyed.

So, as protection, one of the $D p n I I$ restriction system enzymes, a methylase, first methylates the adenine of every GATC sequence of the cellular INA. The DpnII system endonuclease is then free to attack the GATC of the viral DNA.

The complementary teamwork of DpnI and DpnII seems to have evolved as a way to ensure survival of the species during epidemic infections by viruses. As yet, it is not clear exactly how these restriction enzymes are regulated. How does the cell know when to produce a particular enzyme, and why do the other components remain inactive at the appropriate time?

Such questions are being answered in our continuing exploration of these enzyme systems. By 1986 , we had identified all the bacterial genes making up the $D p m I$ and $D p n I I$ svstems and determined the sequences of their DNA bases. By 1987 , two of the enzymes in these systems were crystallized, making it possible to determine their structure three-dimensionally.

Further work is focused on showing how these enzymes interact with the INA that they recognize. where the interactions are. and how the chemical reactions take place. Isuring our research, more of the secrets of the S. premmerniare "hatchet men" will comme to light. 


\section{Support Activities}

At Brookhaven, new ideas and new techniques are actively pursued, as we continuously probe the forefront of science and technology. Critical to the success of the Laboratory's scientific programs are activities in the Applied Mathematics Department, the Reactor Division, the Safety and Environmental Protection Division, and the Instrumentation Division. The following pages describe each organization and focus on special projects carried out this year.
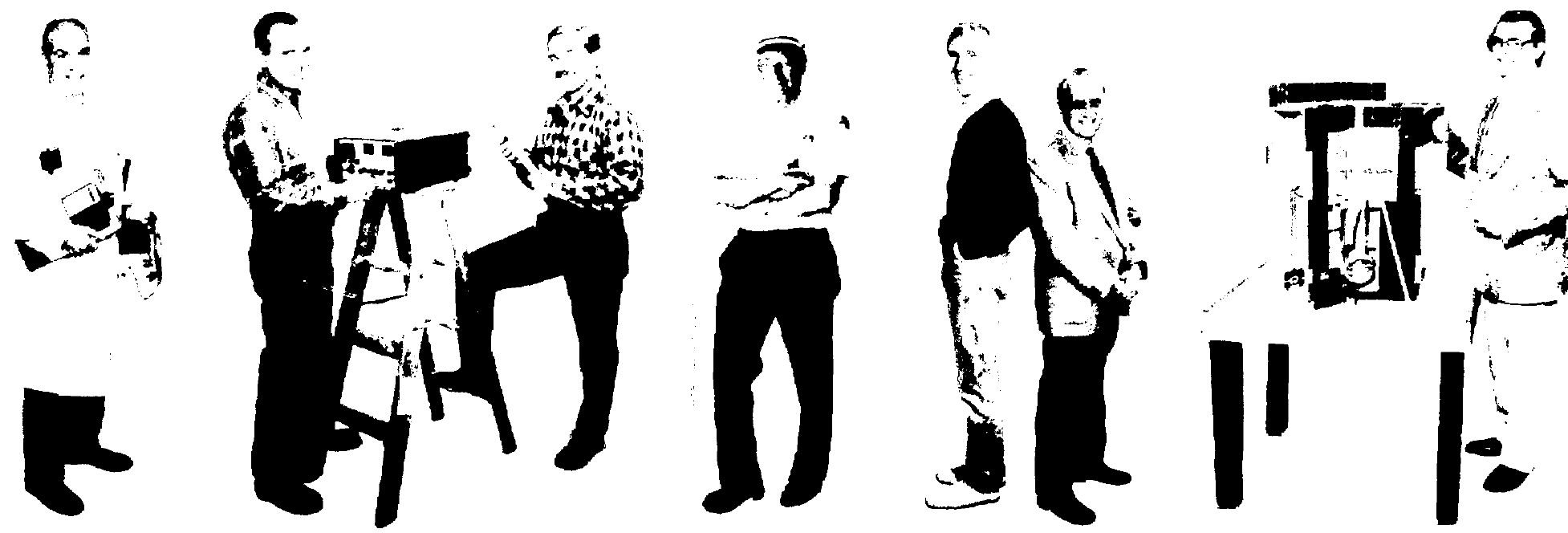


\section{Applied Mathematics Department}

In July 1962, in answer to changing needs of the Laboratory, Brookhaven's Applied Mathematics Division became the Applied Mathematics Department. Since then, work has encompassed a broad range of basic research in mathematics, statistics and computer science, and the operation, planning and maintenance of BNL's major central computers and communication networks. In October 1988, again reflecting current trends in computer science, the department divided into two: a mathematical research group which joined the Department of Applied Science, and the new Computing and Communications Division.

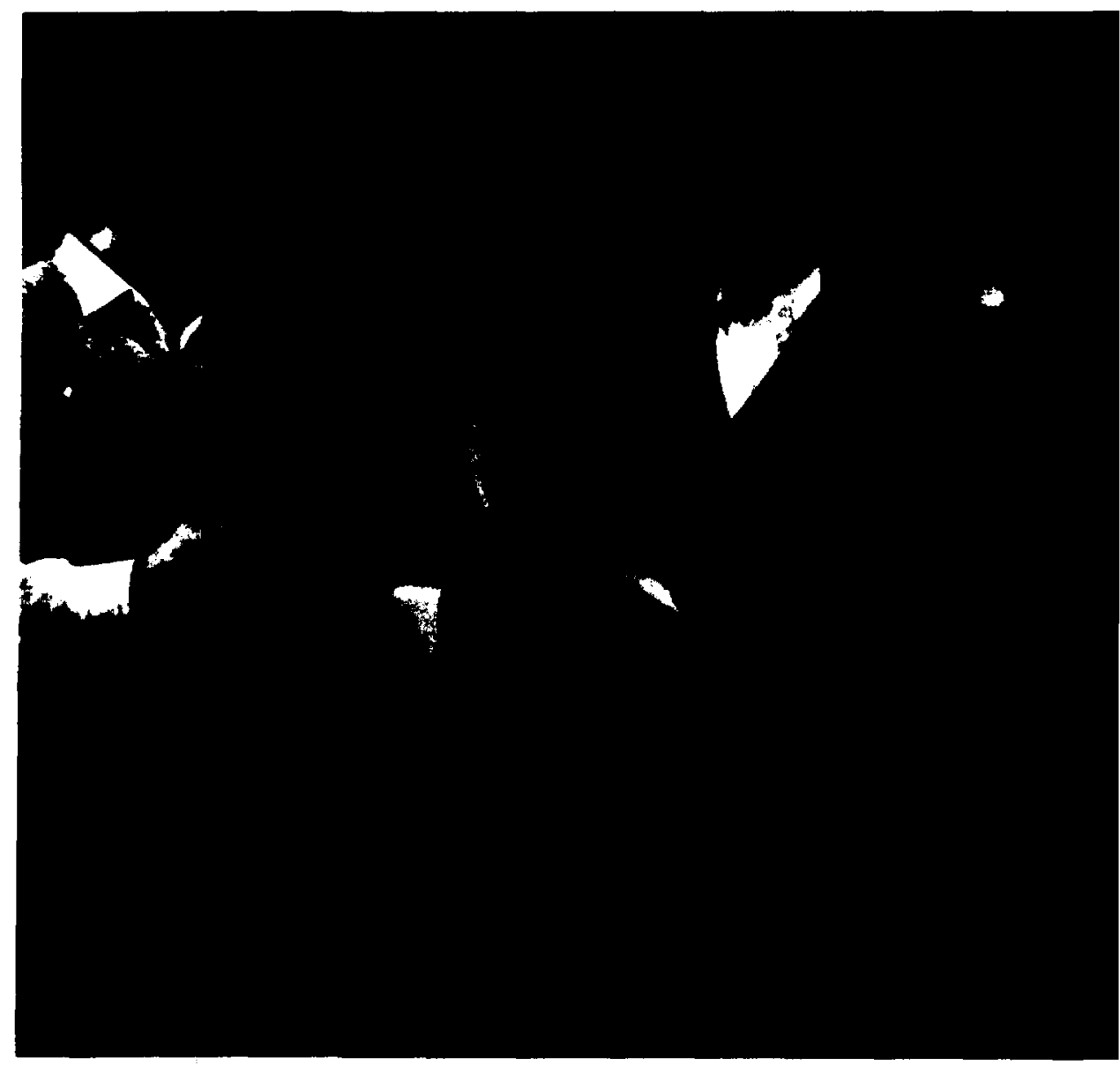

The unobtmesive giant in the background is the IBM 3090, Brookhaven's most. super-poulerful new computer. Seated at the terminal of the computer, Susan. Sevian discusses instructions from a manual urth (from left) Barry Arbeit and Graham Campbell. 


\section{Multiplication Leads to Division}

On October 1, 1988, as a result of multiplication of its computing functions over the past years, the Applied Mathematics Department (AMD) divided into two parts.

AMD's Networks, Engineering and Telecommunications Division, together with the Computing and Communications Division, became Brookhaven's new Computing and Communications Division, responsible for all computing, telecommunications, computer networking and engineering support functions.

At the same time, AMD's principal research function, the mathematical research group, joined the Department of Applied Science as a new Mathematical Sciences Division. Here, researchers will establish collaborative activities with others also working on basic science programs applied to practical problems.

This administrative change reflected the strong growth over the past 25 years at Brookhaven of computing and communications technologies, which are vital to scientific programs. When AMD was first formed in 1962, computers in science were special tools that few people knew how to work. Our early computing equipment was in a central location, where scientists could come for assistance with problems in numerical computations. Research in mathematics and the computing facilities were natural partners.

Today, one research activity involves the study of nonlinear dynamical systems. These systems can frequently behave in a chaotic manner. That means that each individual piece of the system's motion appears unpredictable and erratic, but that the overall pattern of motion has well-defined structure.

To examine such problems, graphic techniques are essential. Sophisticated computer software

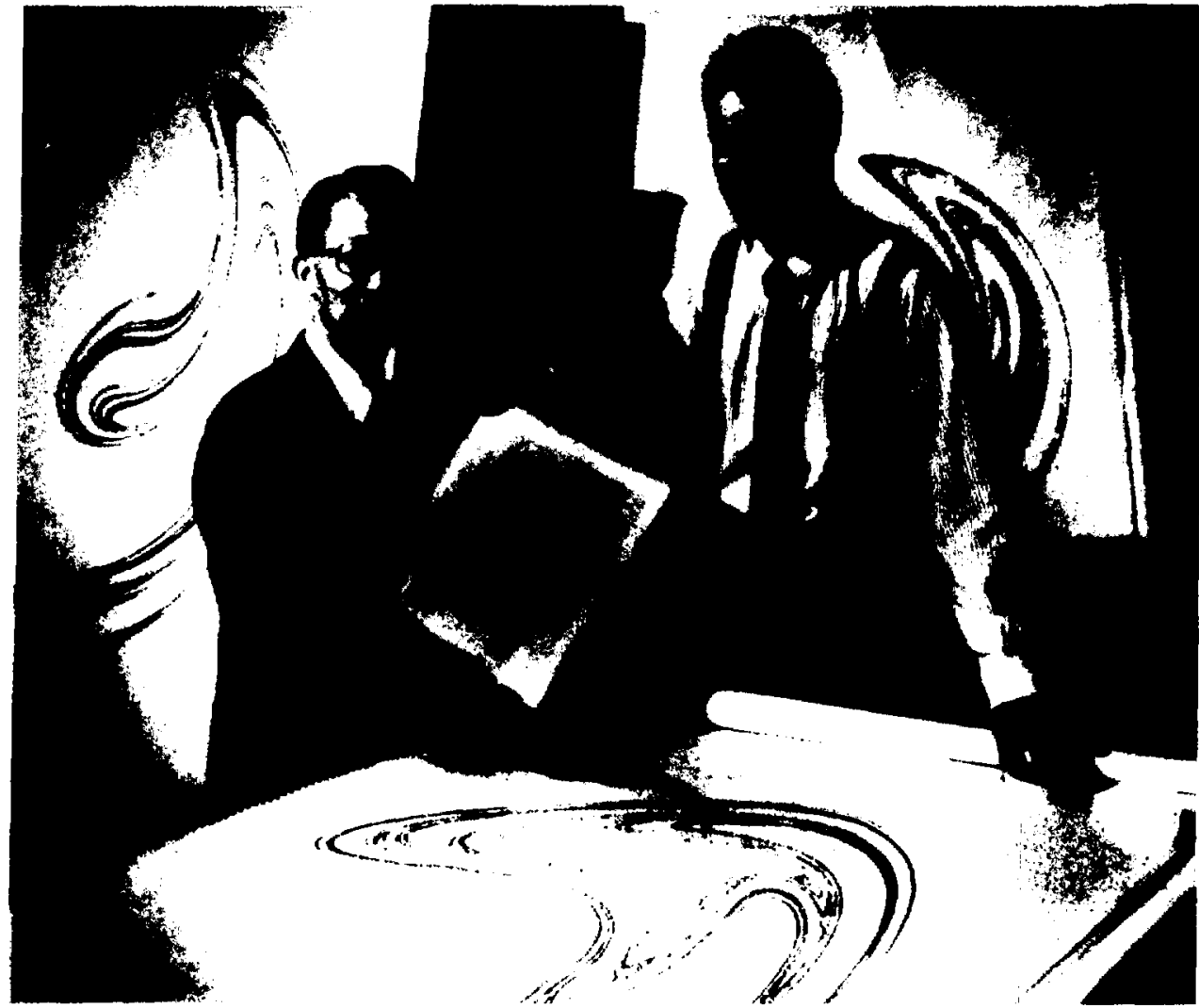

Science became art in the display of original drawings and computer plots exhibited in September 1988 at Brookhaven in "Portraits of Chaos" by Yoshisuke Ueda (left), from Kyoto University in Japan. Ueda has collaborated on chaos research urith BNL's Bruce Stewart, who gave a lecture on dynamics to explain the exhibition.

has been developed to allow the mathematician to do experiments on the behavior of interesting systems. Some of the images produced in the course of this work have an aesthetic appeal quite apart from their scientific interest. In fact, the work led to the presentation of an art exhibit combined with a dynamics lecture, using the work of a Japanese collaborator who is a pioneer in this field.

Other activity in the mathematical sciences is connected with the development of algorithms (procedures for solving mathematical problems) that can take advantage of parallel computers, which are rapidly increasing in capability and availability. The goal is to find procedures that can be divided into independent pieces that can go forward simultaneously, no one piece requiring the solution of any other one to proceed.

Most recently, Brookhaven researchers are exploring the field of asynchronous parallel computing. This method differs from parallel computing in that the various subtotals of information are added into the total not all at once, but as they are ready. Therefore, depending on the problem, it could happen that a quickly finished subtotal of knowledge might affect the subtotal of another part of the problem by being added into it before it was resolved independently. So, with a lack of organized synchronization, the way is open for completely new effects to be discovered that may revolutionize computational capabilities in the future.

With so much that is new in com puter activity, and so many compu- 
ter users at Brookhaven and elsewhere, it is easy to see why technical support for computing and communications has become large enough to warrant a separate division to manage the effort.

A major effort for the newly formed Computing and Communications Division (CCD) is the support of a wide range of specialized networks available to Brookhaven scientists. On site, CCD will continue to "bridge" custom-built networks in facilities like the Alternating Gradient Synchrotron and the National Synchrotron Light Source with other networks on site. The local networks that will be serviced by the division have already added miles of fiber-optic cables to existing lines a firm basis for the next generation of fiber-optic lata pathways.

Brookhaven's wide-area networking includes, for example, HEPnet and BITnet, which link high energy physics users and other Laboratory users both nationally and in Europe; TELEnet, another communications service with Europe; and MFEnet, which ties users into the Lawrence Livermore National Laboratory in California.

Our most recent installation allows us access to NYSERNet, the New York State Education Research Network. NYSERNet links Brookhaven with several industrial laboratories and 14 universities, includ-

\section{Reactor Division}

The Reactor Division operates one of the world's most advanced research reactors, the High Flux Beam Reactor (HFBR), as well as the Brookhaven Medical Research Reactor. The HFBR's intense beams of neutrons support experiments in nuclear and solid state physics, metallurgy, nuclear and structural chemistry and biology. The tradition of outstanding research developed by Brookhaven researchers and the international community of scientists working there includes award-winning work on such topics as behavior of metals and ceramics, and high temperature superconductor systems.

\section{Spin in Superconductors}

\footnotetext{
The good news was: Significant advances in high-temperature superconductors were made in 1986 by the international physics community. These ceramic mixtures of rare earth material, copper and oxygen conduct electricity without
}

ing Cornell with its supereomputer, which, in turn, is linked to four other supercomputers (and their attendant networks) across the country.

In providing technical support for these networks, along with other tasks such as operating the Central Scientific Computing Facility and the Laboratory's telephone system, lending programming assistance, and planning and developing future data processing facilities and tools, the CCD is responsible to a very large extent for the smooth running of many important areas of Brookhaven. losing energy - below critical temperatures $\left(T_{c}\right)$ high enough to be affordable. When these new superconductors came on the scene, their applications in industry, such as computers operating a hundred times faster than those now available, or electric power transmission at much diminished cost, seemed just around the corner.

The bad news is: The nature of the new materials prevents their being applied in many practical ways. For example, they are too granular to be formed into long wire shapes. Some method has to be found to change either the ingredients or their proportions so that they remain superconducting, yet are easier to use.

The first step is to understand how the high- $\mathrm{T}_{\mathrm{c}}$ superconductors work. Researchers all over the world began exploring different paths along this new frontier of scientific discovery - and Brookhaven scientists were no exception. Among the programs of investigation started here (see related story page 31 ) were those at the High Flux Beam Reactor (HFIBR). 


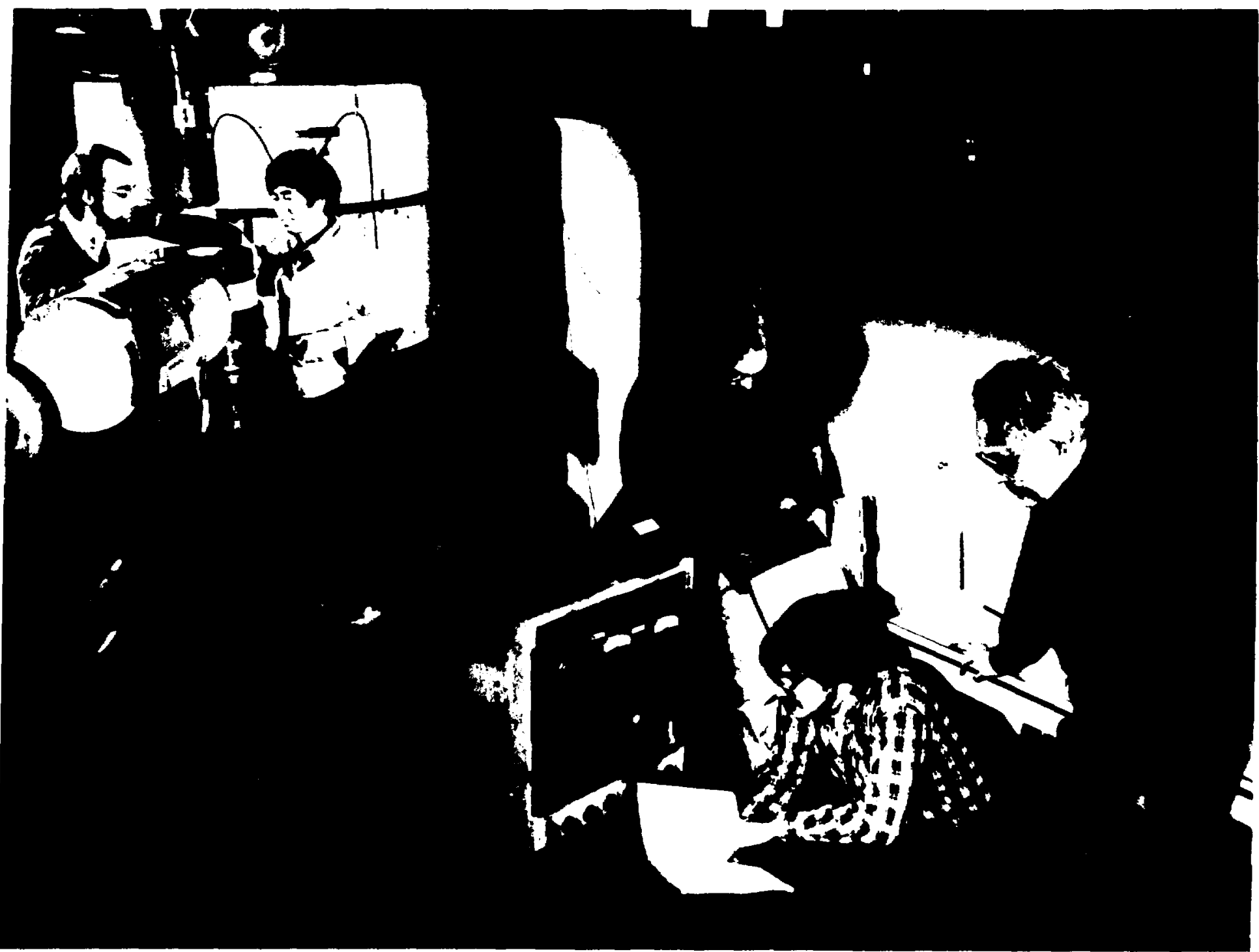

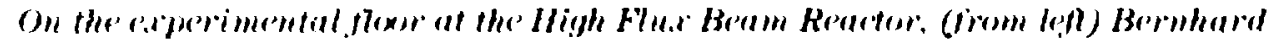

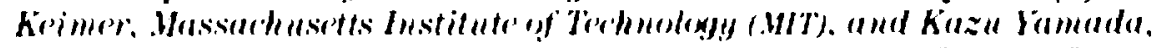

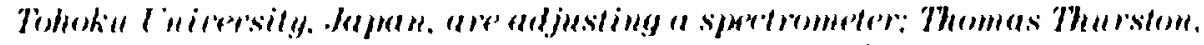

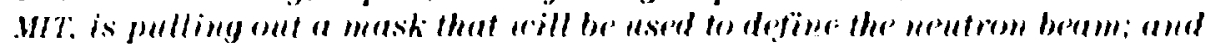

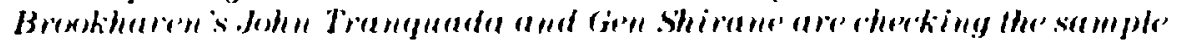

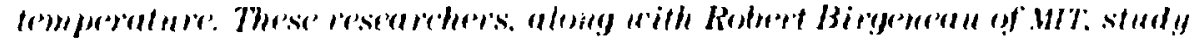

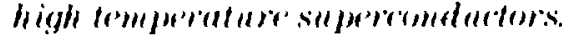

Browkhascolis HFHR is a souree of particularly pure and incense ther mal netutrons, which are ideal for neutron diffiatetion experiments that look into the matghetic propere liess of individual atoms in a mate. rial. Through magnet ic seat tering studies. the magnitude and direstion of the individual atomis spins. as well dis the alignment of all spins: ill a staterial, ran be learned.

One team of experimenters. which included seient ists from Brookhaven. the Massachusefts Institute "f Terohology, and Tohoku l miversily in .Japan, loegan st udying spin dynamies in high- $\mathrm{l}_{6}$ stuperentuduetor sistems. Theif prospess was olltst anding and gained them a place as one of the two researeh trams at Brookthaven, both working at the HFBR. that were among the nine winning 1 (ams of the I lepart ment of Energy lase Materials Sorences Roseareh competition. Therir discoveries an spoin elymanios prover eompat ible with part of the theory proposed by Bronkharem phisidojsis on how' supereonductors work.

'lhis theory, like many ot her's. refuires the presence of ant iferonmagnetic cormelations belween the copper atoms in the copper-oxygen planes that are a common strucetural element in supereonductors will T. groalce than :30 kelvins. Antiferromagnotism ocours when the magnetice moments. or spitss, of neightoring aloms point in ofporste directions, alternalling in all updown-up-down array.

And this is just what the lescarchers saw. In a neutron dil'fract ion exporiment. they directly observed the lineup of opposite ()ㅏeㅏ spins in single clystals of lanthantum-(e)pler-oxigen, the pall ent compound of ons family of high $\mathrm{T}_{f}$ supereconductors. 
The neutron beam in this first experiment was unpolarized; that is, the spins of the neutrons were not oriented in a particular directon. Therefore, the antiferromagnetic characteristics it produced could possibly be explained by another phenomenon. So, the team continued their studies with a polarized neutron experiment, in which the majority of neutrons have their spins pointing in one direction.

This second experiment showed that, again, the spins of the copper atoms alternated, over a relatively long range, verifying a point fundamental to the Brookhaven theory.

More experiments on the magnetic behavior of high- $T_{c}$ superconducting compounds were performed. They revealed that the magnetism is strongly two-dimensional and that copper and oxygen atoms couple with unusual strength in planes within the compound.

Further, the two-dimensional, antiferromagnetic correlations survive in the absence of long-range order.

One important discovery made by the team led to new physics. By using a technique called inelastic neutron scattering, the researchers could see that above the temperature at which long-range ordering occurs, groups of copper spins in the same plane were oriented antiferromagnetically, but that their spin direction fluctuated very rapidly.

Thus, a snapshot of the spins showed antiferromagnetic correlations, but a time exposure showed the fluctuations, which appear as complete disorder. This dynamic antiferromagnetic behavior in the high- $T_{c}$ phase of lanthanum-copper- oxide has been called a quantum spin fluid.

As a result of these experiments, the researchers learned that the dynamic correlations of spin direction were due to the extremely strong magnetic coupling between neighboring copper atoms, a coupling that is mediated by intervening oxygen atoms. This was especially interesting, as the large interaction energy is crucial to Brookhaven theory of the superconducting mechanism - there is a strong relationship between the antiferromagnetism and high- $\mathrm{T}_{\mathrm{c}}$ superconductivity phenomena.

Further research is already under way at the HFBR to explore other superconducting compounds in the light of these discoveries.

activities. By the end of the year, Brookhaven had issued its first Scientific Progress Report. Contained within was a section reviewing procedures for handling hazardous wastes generated from our research programs.

Thus, from the very beginning, the Laboratory has cared about the environment in which we reside. We recognize the potential for problems with hazardous waste disposal, and we take steps to avoid such problems.

Brookhaven has also been open with the public and regulatory agencies about the effects of its operations on the environment. One way this has been done is through environmental reports - public documents that have been issued since the Laboratory's early years. This information is sent to federal and state agencies, as well as to Suffolk County, which has been con-

\section{When Brookhaven was established in 1947, the site looked no different from the Army camp it had once been. Within months, though, old}


ducting environmental inspections of the Laboratory since March 1988, as the result of an agreement signed in September 1987 between the county and Brookhaven.

The Laboratory's extensive program of environmental monitoring and protection is conducted by the Safety \& Environmental Protection Division (S\&EP). Its purpose is to ensure that all Laboratory operations are conducted safely and with minimum effect on the environment. The program includes monitoring all Laboratory releases to air and water, as well as assessing the impact of Laboratory operations on the local groundwater and the nearby Peconic River.

S\&EP keeps a watchful eye on potentially harmful materials that are chemically toxic, radioactive, or a mixture of both. Reviewing the environmental monitoring data collected over the past four decades, our record shows that the Labora- tory has always operated well within applicable laws of the time.

Through the years, however, regulations have become increasingly stringent, and wisely so, particularly for Long Island, which relies on a sole source aquifer for its drinking water. So even though we have acted responsibly in the past, today's stricter regulations call for certain past actions to be corrected in the present.

An example of this is the use of degreasers. For many years, degreasers containing the solvent trichloroethane (TCA) were the substances of choice, and people all over Long Island - including at Brookhaven Laboratory - used these commercially available products freely and disposed of them without restriction.

The uncontrolled disposal of TCAcontaining degreasers came to a halt in the early 1980's, when federal and state authorities began

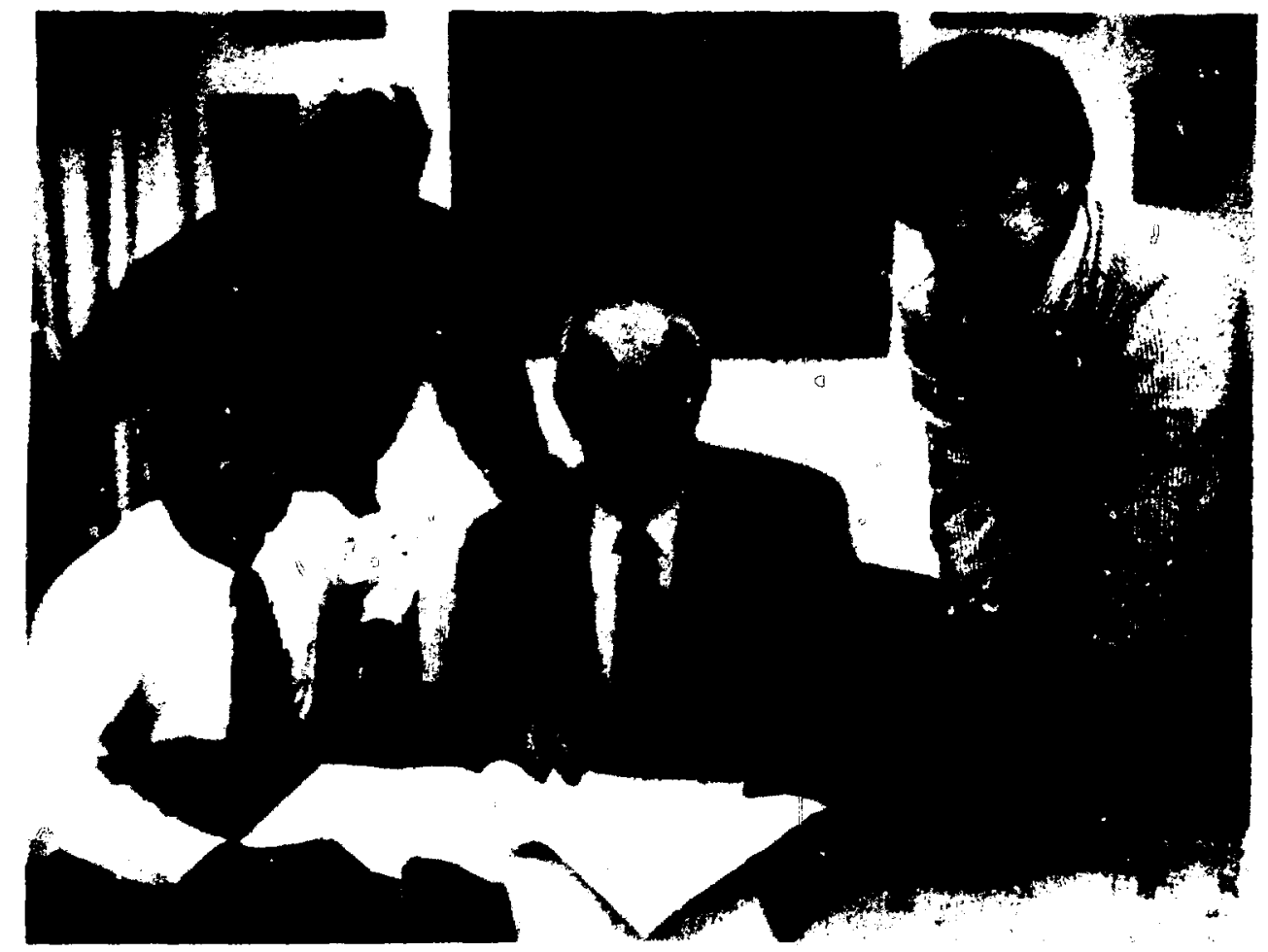

Discussing Brookhaven's programs for monitoring the environment are (from left) Robert Casey, Head of the Safety \& Environmental Protection Division (S\&EP); Janakiram Naidu, who oversees the division 's environmental protection section; Gerald Kinne, BNL's Assistant Director for Reactor, Safety \& Security; and Leonard Emma, who heads S\&EP's hazardous waste and emergency preparedness section. regulating the substance after the discovery that compounds similar to TCA might be carcinogenic. Though it continues to be used at Brookhaven, we now follow strict regulations for its disposal in order to prevent TCA from entering the environment.

As good as these measures are, however, they can do nothing to correct the problems created before the current restrictions on TCA were established. In 1984, concentrations of TCA higher than current drinking water standards were found in the groundwater during routine sampling of test wells near the waste management area, where hazardous waste is packaged before being taken off the Laboratory property for disposal at federal sites.

A consulting firm hired by S\&EP traced the TCA plume by putting in wells and analyzing groundwater samples. They found concentrations up to 1,800 parts per billion (ppb); the legal limit is $50 \mathrm{ppb}$. The plume had traveled about 2,000 feet to the southeast, but had not affected the Laboratory's drinking water and had not reached the site boundary. To see that it progressed no further, Brookhaven began a remediation program in April of 1986.

The technique employed in this project takes advantage of the fact that TCA, an organic compound, is hydrophobic, so it readily separates out of water vapor sprayed into the air, where it is far more easily diluted. This method of decontamination is the preferred process for cleaning TCA-contaminated groundwater and has been frequently used on Long Island.

Comparisons between the water before and after spraying show that we are removing TCA with 95-100 percent efficiency. Already, the TCA concentrations have dropped to about $100 \mathrm{ppb}$. The program will continue until the concentration is reduced well below the regulatory standard.

The handling, storage and disposal of hazardous material is 
highly regulated at county, state and federal levels. But, in disposing of hazardous materials, Brookhaven would like to be better than the law requires. For this reason, we follow the ALARA principle. ALARA stands for the concept "as low as reasonably achievable."

Hazardous wastes are not only costly to the environment, they are also a drain on the Laboratory's funding for research. Through 1990 , Brookhaven expects to spend over $\$ 17$ million to correct problems, such as the TCA contamination, that evolved under years of less stringent regulations.

Due to the nature of Erookhaven's research, hazardous materials will always be a part of the Laboratory scene. But by carefully monitoring the environment, by following regulations and by encouraging the entire Brookhaven community to practice ALARA, we can minimize the environmental and monetary costs of hazardous waste management.

\section{Instrumentation Divison}

Breaking new ground in scientific research requires new concepts, new theories - and the appropriate instruments to enable them to be developed. The primary activity of the Instrumentation Division is to provide new tools and methods for the high precision measurements needed in experiments of all branches of science, and in detection of particles and photons in particular.

New techniques are being developed and applied in the areas of low-noise hybrid and microelectronic circuits and laser-based instruments in the picosecond domain. Special services in such areas as vacuumdeposition technology, optical metrology, electron microscopy and printed circuit board fabrication are also made possible by the expertise of division staff.

\section{Thinking Tiny}

A grating can come in any size. It can be large enough to swallow a set of dropped car keys, or small enough to grind nutmeg. If it is microscopically small, it could be one of the gratings being designed and made in Brookhaven's Instrumentation Division.
Here, materials scientists are at work on structures so fine that a single human hair is ten times thicker than the spaces in the grating. These microscopic structures will be a key element in a totally new concept for a linear accelerator, one that will accelerate electron beams to ultrahigh energies much more efficiently than is possible with current technology. 
In the Accelerator Test Facility now nearing completion, a narrow electron beam will first be preaccelerated conventionally to 50 megavolts (MeV). The beam will then be focused to skim just above the grating, which is being irradiated by a high-powered carbon dioxide laser. The intense electric field resulting from the grating-laser interaction will accelerate the electrons in the beam. The amount of energy gained by the electrons will then be measured in a spectrometer.

A crucial part of the concept is the design of the grating. First, its "periodicity" (the distance between the ripples in the grating) must be matched to the laser's wavelength of 10.6 microns, or

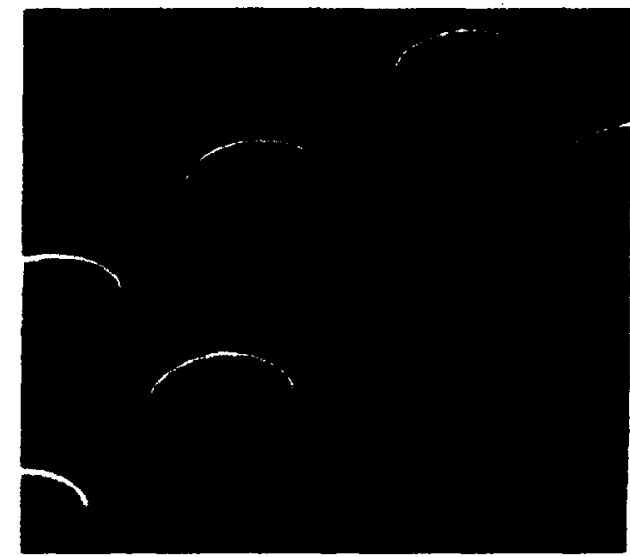

The mask consists of an array of oxide disks on a silicon substrate that forms the final grating after ion etching.

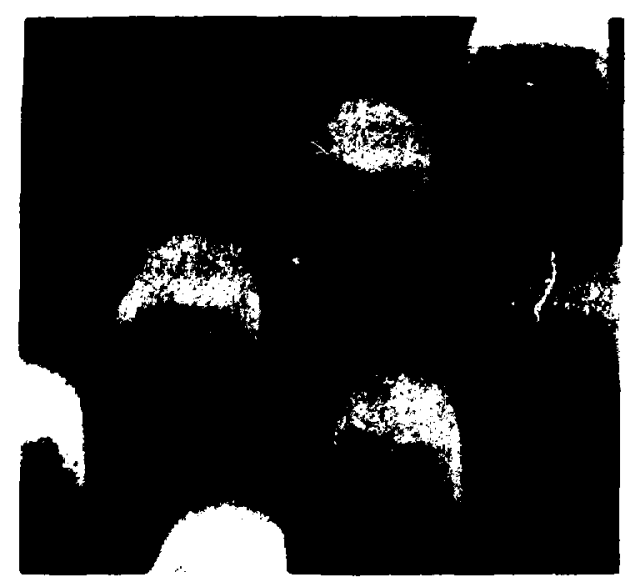

The finished grating, magnified 6,000 times.

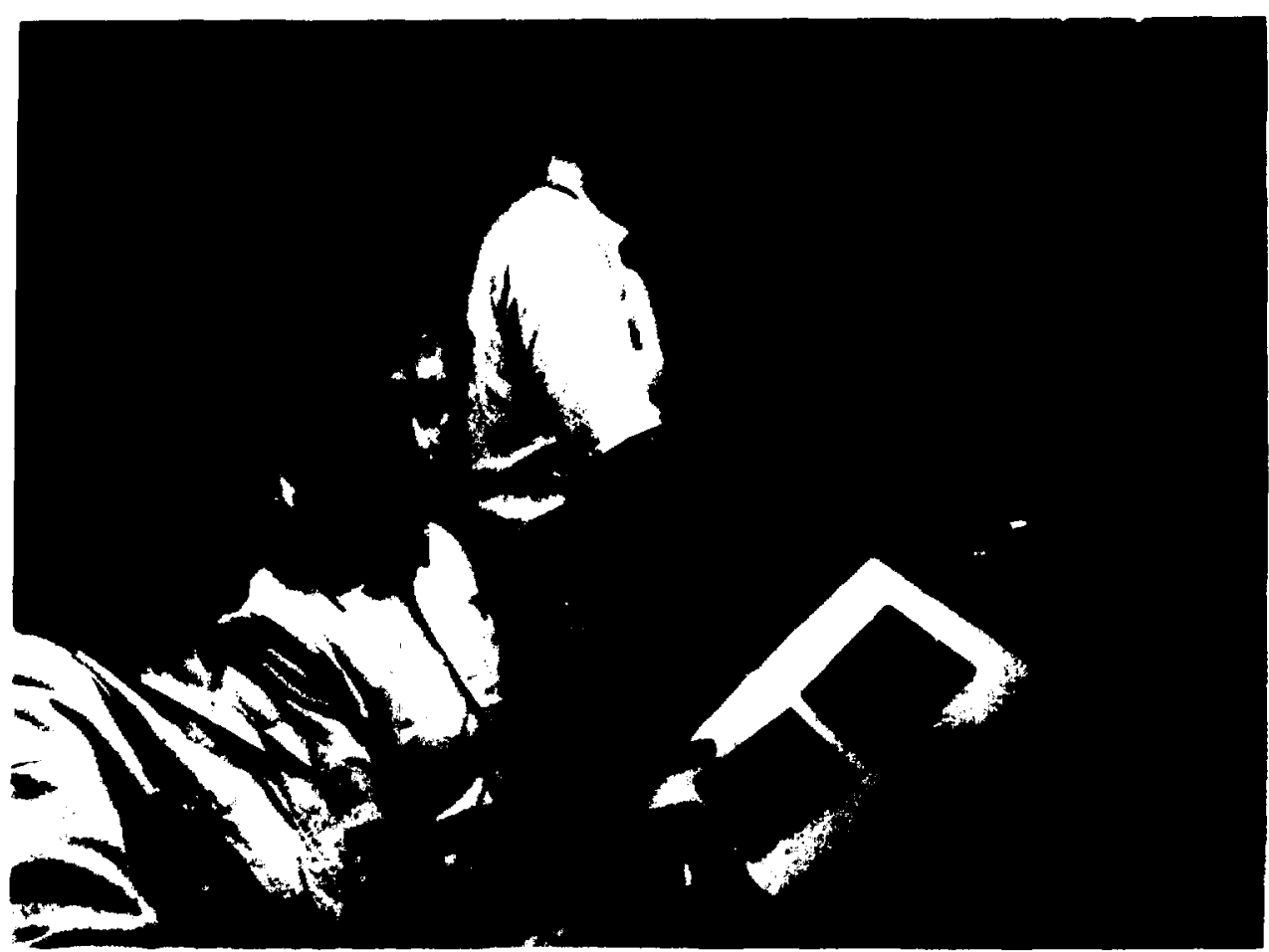

While.James Calcanes (standing) prepares the electron microscope for use, Mara Schuartz, of North Carolina State University, and John Warren compare the greatly magnified micrographs of the completed gratings urth the examples they are holding, in which the actual distance from center to center of the upright components is about one-tuentieth of the diameter of a human hair.

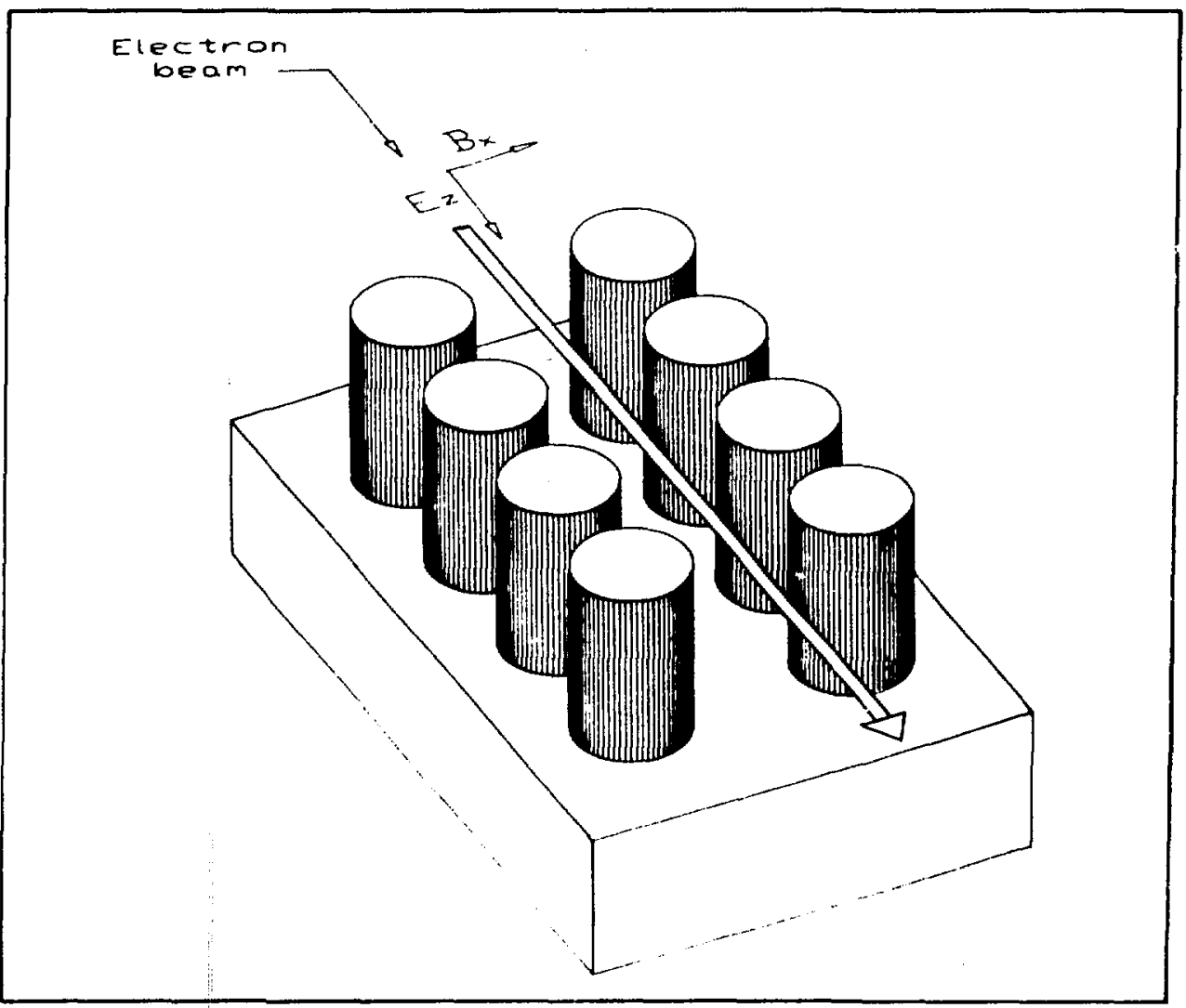

The idealized desigm shous the path of the elertrom bram as it passes onwr the yrating. 
about $1 / 3,900,000$ of an inch. By using computer simulations of the interaction between the electric field around the grating and the electron beam, researchers have calculated the most effective shape and dimensions to use.

Two possible grating designs are under consideration. One type is a double row of cylinders that project a few microns above an optically flat surface. The other is a row of linked rectilinear cavities of similar dimensions.

If the grating's dimensions do not conform with complete accuracy to the dimensions specified by computer simulations, the energy from the laser will not couple efficiently to the electron beam, and the electrons will not be accelerated.

The designers must take into account other, equally stringent, considerations. The material used to make the grating has to be conductive, so that energy losses through resistance are reduced. It must also be extremely resistant to damage from the high-power carbon dioxide laser.

The dimensions of some possible grating designs have been worked out, trial materials selected and several prototypes produced. The procedure for making the grating is similar to the lithographic process used in the manufacture of integrated circuits in computers.

An electron-beam writing instrument is used to draw a twodimensional representation of the grating pattern on to a photoresist layer that overlays a substrate of the chosen material. Then a process known as reactive ion etching is used to etch selected regions not protected by the masking pattern. The grating is formed after the top two of three microns of substrate have been etched away.

The methods of measurement used at present in the semiconductor industry are based on optical microscopy and do not have sufficient resolution to measure the gratings. Brookhaven scientists are therefore developing equivalent methods that use scanning electron microscopes or scanning tunneling microscopes. These instruments have much greater resolution and can provide the extreme accuracy needed.

A few millimeters of grating sections are expected to be completed by the end of 1989 , and the grating accelerator concept can then be fully tested. If it succeeds, a new frontier will be opened in advanced accelerator research. 


\section{Laboratory Profile}

Brookhaven's multidisciplinary nature was reflected in the wide range of scientific meetings hosted here during fiscal year 1988. The same breadth of research is reflected in the variety of honors and awards garnered by so many of our employees. And within the scope of general and administrative activities, a number of new programs were initiated in such areas as training, team safety and energy conservation, all part of the busy, day-to-day life at the Laboratory.
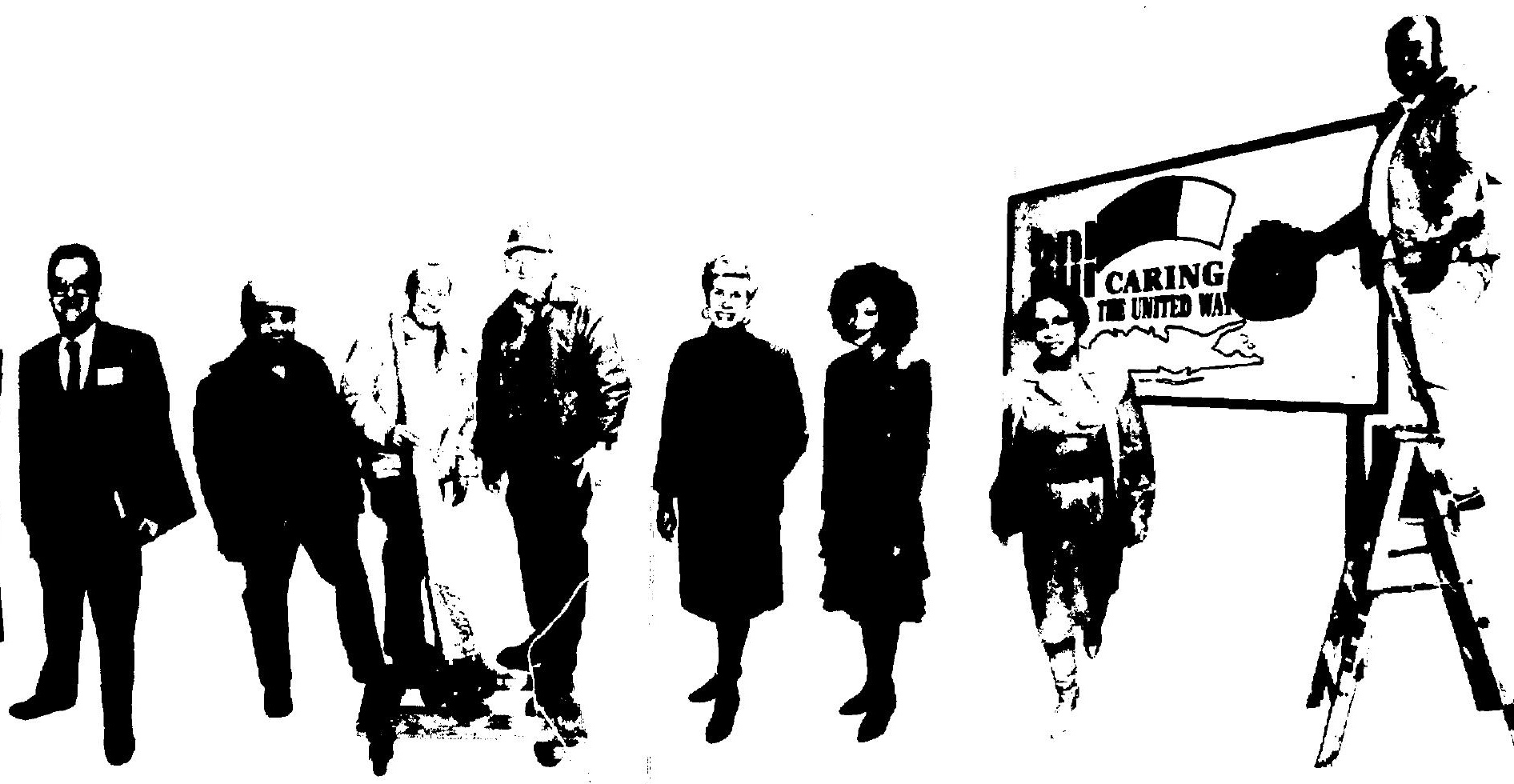


\section{Mering}

On almost any day of the year, Brookhaven is hosting a conference, workshop or symposium. This sample shows how the dozens of meetings held at our multiprogram laboratory in 1988 appealed to a wide variety of scientific interests.

Electroresponsive polymers include electronic and ionic conductors and non-linear optical materials. In the last ten years, they have emerged as a major interdisciplinary field of study. For this reason, some 80 scientists attended the Symposium on Electroresponsive Polymers, held October 4-7.

Worldwide, a total of eight nuclear data centers have joined in agreements to exchange nuclear reaction data. Representatives of these centers gathered at the National Nuclear Data Center at BNL, October 27-29, for the ninth in a series of biennial meetings sponsored by the International Atomic Energy Agency in Vienna.

For the first time ever, chemists specializing in synchrotron radiation research - about 120 in all were brought together November 46 for a conference entitled New Opportunities in Chemistry: An International Symposium on the Uses of Symchrotron Radiation in Chemistry.

Some 80 scientists and physicians gathered at the Second International Symposium on Genetic and Salt Hypertension, November 12-13. Basic and clinical aspects of hypertension were discussed, and further studies were planned using Dahl rats, two special strains originally developed at BNL.

On December 3-4, the National Fire Protection Association conducted a seminar, attended by about 50 Laboratory employees involved in building fire safety, on the Life Safety Code, which deals with safe means of exiting buildings and with general fire safety.

Recently, neutron capture therapy has been refined to the point where clinical trials on this method for destroying tumors may soon begin on brain tumor patients in the U.S. This progress was among the topics discussed by an international group of 75 medical and scientific specialists, at the Neutron Capture Therapy Workshop, February 1-2.

To review progress in the analysis of international electricity exchanges - particularly the export of additional electricity from Quebec to the U.S. - 31 researchers and analysts representing governments, academic institutions and the electric power industry met at BNL April 5-6 for the Workshop on Modeling of International Electricity Exchanges.

Sponsored by Brookhaven Women in Science, the Nuclear Structure Colloquium and a testimonial dinner were held April 14 to honor Gertrude Scharff-Goldhaber, retired Senior Physicist and now a research collaborator, for her contributions to nuclear physics and to advancing the cause of women in science.

On May 4, a workshop was held on studying phenomena in nonlinear optics, medicine and surface science, using the infrared free electron laser facility to be constructed at Brookhaven at the Center for Accelerator Physics.

Many of the almost 700 researchers who do experiments at BNL's Alternating Gradjent Synchrotron (AGS) were on hand May 5-6 for the AGS Users Group annual meeting, to hear riports on the accelerator's broad and active research program and new experimental results.

The conference on Vacuum Design of Advanced and Compact Synchrotron Light Sources, which was sponsored by BNL and the Vacuum
Technology \& Surface Science Divisions of the American Vacuum Society, was held May 16-18 and drew 150 participants.

Synchrotron Radiation in Structural Bjology was the title of the 35th Brookhaven Symposium in Biology, held May 22-25. In addition to reviewing results obtained by synchrotron-based crystallography, small angle scattering and spectroscopy, the 60 participants explored instrumentation improvements and new ways of using synchrotron radiation.

The fundanıental physics and chemistry of solid surfaces and interfaces was the main theme of the 48th Physical Electronics Conference held June 6-8, which was cosponsored by BNL and the American Physical Society and attracted close to 200 attendees.

The Summer Institute on Relativistic Heavy Ion Physics had two parts: Attended by 115 students, a summer school was held July 11-15, to attract young physicists to the field of heavy ion physics, while about 130 attended a workshop on detector technology for BNL's proposed Relativistic Heavy Ion Collider (RHIC), July 18-22, to draw up a plan for detector R\&D and begin writing proposals.

At an August 15 workshop, 20 attendees discussed the future of health physics education and BNL's role in providing training for health physics master's degree students.

Over 110 physicists met at Brookhaven August 25-27, for the Workshop on Rare $K$ Decays and CP Violation, where experimenters and theorists discussed the present status of experiments in these areas and where to aim in the future.

As one of the three collaborators in the magnet design for the proposed Superconducting Super Collider (SSC), on August 3I, BNI. was 


\section{Fonors}

Per Bak (Physics) was elected a Fellow of the American Association for the Advancement of Science (AAAS) in February, for his contributions to solid state physics.

Robert Birgeneau (guest, Physics) and Paul Horn (guest, National Synchrotron Light Source) were awarded the American Crystallographic Association's 1988 Warren Award in June, for their work on a series of experiments on diffraction from two-dimensional systems, in which they used the Light Source.

Brookhaven won a Department of Energy (DOE) Small Business Award for using small and disadvantaged businesses, having had contracts with 75 such firms in 1987.

Brookhaven's 40th anniversary publication, The First Forty Years, received a first-place Distinguished

Brookhaven's Joseph Wall (right) is congratulated by Secretary of Energy John Herrington at an awards ceremony in Washington, D.C., held on September 20 for the six winners of the 1988 Ernest Orlando Laurence Memorial Award. Wall, a biophysicist, is internationally recognized for his piomeering work on the development of the Scanning Transmission Electron Microscope, which is powerful enough to see the fine structure of biological specimens, including single atoms.
Technical Communications award from the New York Chapter of the Society for Technical Communication (STC), in April. In May, the book captured a fourth place Award of Achievement in the STC's 1987 International Competition.

The Brookhaven Bulletin, the Laboratory's employee publication, was honored in April with an Award of Excellence, the STC's secondplace award.

Jerry Cadwell (Nuclear Energy) and Douglas Humphrey (Photography \& Graphic Arts) won a firstplace award at the annual Nebraska Videodisc Symposium in October, for their videodisc "The Authorized Classifer."

Neal Carciello, Lawrence Kukacka and Toshifumi Sugama (Applied Science) earned an R\&D 100 award in September, for one of the year's 100 most significant inventions - a zinc-phosphate coating system that greatly reduces corrosion of steel surfaces.

Ernest Courant (Accelerator Development) received an honorary Doctor of Science degree from Swarthmore College in May.

Carl Dover (Physics) was awarded an Alexander von Humbolt research fellowship in March, to spend a year doing research on theoretical nuclear physics at the University of Tübingen , West Germany.

Joanna Fowler and Alfred Wolf (Chemistry) shared the 1988 Gustavus John Esselen Award for Chemistry in the Public Interest, in March. They are well-known for their pioneering work on positron emission tomography, a technique that uses

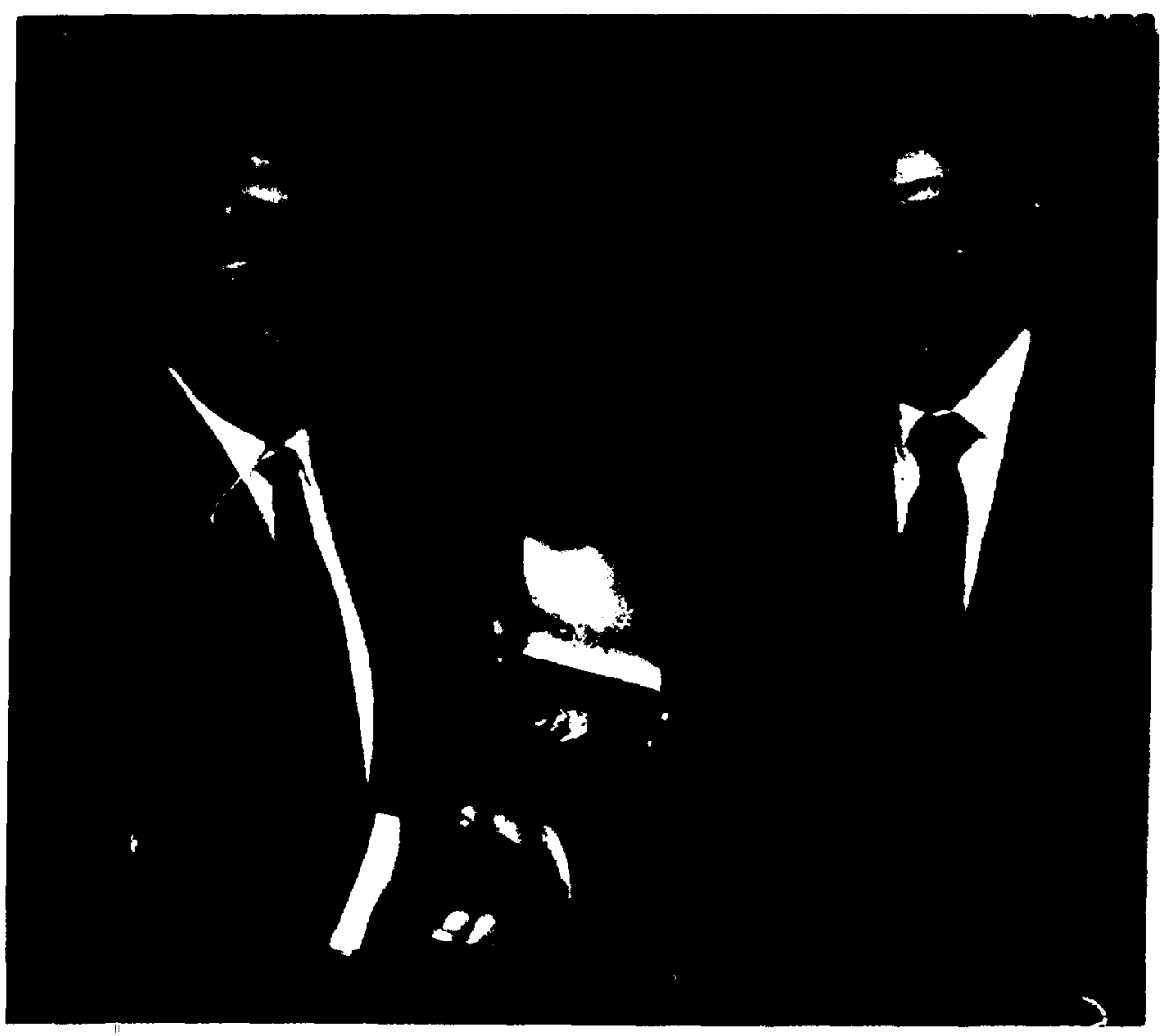


radiotracers to study organs and metabolic processes in the human body.

Ole Hansen (Physics) was awarded an Alexander von Humbolt research fellowship in May, to spend ten months at the University of Frankfurt, West Germany, collaborating on the NA35 experiment at CERN, then two months at the nuclear physics accelerator laboratory $\mathrm{KV}$, in Groningen, the Netherlands, working at the heavy ion cyclotron.

Willi: a Higinbotham (Nuclear Energy) was honored in September when the library for the Technical Safeguards Organization in BNL's Department of Nuclear Energy was formally dedicated as the "Higinbotham Nuclear Safeguards Library."

Keith Jones, Barry Gordon, Albert Hanson and Joel Pounds (Applied Science), in collaboration with Mark Rivers, Stephen Sutton and Joseph Smith (guests, Applied Science), were honored in September with an R\&D 100 award for developing an $x$ ray microprobe/microscope that can determine the specific chemical compositions of geological and biological samples.

Joseph Kenneally (Contracts \& Procurement) won the first BNL Small Disadvantaged Business Standard of Excellence Award in March, as the quarter's buyer with the best performance in small and disadvantaged business participation.

Richord Lambrecht (research collaborator, Chemistry) was selected by the New Haven Section of the American Chemical Society to receive the Section's 1987 Maurice

R. Chamberland Award in November, in recognition of his application of chemistry to improve the quality of life.

John Larese (Chemistry), Stephen Shapiro (Physics), and Simon Moss (guest, National Synchrotron Light Source), along with their collabora- tors from Lawrence Livermore National Laboratory, were cited for outstanding scientific accomplishment as winners of DOE's 1988 Materials Sciences Research Competition in September, for their studies of "Premartensitic Behavior of the $\beta_{2}$ Phase in $\mathrm{Ni}_{62.5} \mathrm{Al}_{37.5 . "}$

William Marcuse (Office of Research \& Technology Applicatons) was named the Federal Laboratory Consortium's Representative of the Year, in May, for his role in assisting with the initiative to transfer x-ray lithography and synchrotron radjation technology for the commercial manufacture of high-speed computer chips.

Donald Metz (Office of Educational Programs) received the Robert $A$. Leahy Memorial Outstanding Citizenship Award from the Long Island Forum for Technology in May, for the time and energy he has devoted to promoting technology education in the region.

Ronald Rau (Physics) was awarded an Alexander von Humbolt research fellowship in May, to divide his time during 1989 between the DESY Laboratory and the University of Hamburg, West Germany, while participating in the Zeus collaboration, a high energy physics experiment at DESY's electron-proton collider HERA.

Gertrude Scharff-Goldhaber (Physics) was honored for her lifelong contributions both to science and to the cause of women in science, at a Nuclear Structure Colloquium and testimonial dinner in April, sponsored by Brookhaven Women in Science.

Richard Setlow (Director's Office) was elected a Fellow of the AAAS in February, for his research into DNA repair and the biological effects of UV-induced cyclobutane pyrimidine dimers.

Gen Shirane and John Tranquada (Physics), along with Robert Birgeneau (guest, Physics) and Mare
Kastner (guest, Reactor), were cited for outstanding scientific accomplishment in DOE's 1988 Materials Sciences Research Competition in September, for their studies of "Spin Dynamics in High- $T_{c}$ Superconductor Systems."

John Skaritka and George Ganetis (Accelerator Development) earned an R\&D 100 award in September, for developing an instrument for measuring magnetic fields in magnets for particle accelerators.

Suresh Srivastava (Medical) earned a 1988 Special Award for Excellence in Technology Transfer from the Federal Laboratory Consortium (FLC) in May, for his initiative in introducing a red blood cell kit to industrial sponsors and convincing them to commercialize it for use in millions of clinical examinations annually.

Dmitri Stephani (Instrumentation) received a 1988 Special Award for Excellence in Technology Transfer from the FLC in May, for his creativity in inventing hybrid preamplifiers and uncommon initiative in promoting commercialization through assisting manufacturers to produce, advertise and market thousands of units annually.

Michael Tannenbaum (Physics) was elected an AAAS Fellow in Feburary for his proof that muons obey Fermi-Dirac statistics and for his studies of proton-proton and relativistic nuclear collisions.

Gerald Tape (consultant to Associated Universities, Inc.; past AUI President and former BNL Deputy Director) was awarded DOE's 1987 Enrico Fermi Award in December, for his contributions to both the advancement of nuclear power and the nonproliferation of nuclear weapons.

Peter Thieberger (Physics) was awarded a Space Act Tech Brief Award by the National Aeronautics and Space Administration for "the creative development of a technical 
innovation which has been proposed for publication as a NASA Tech Brief entitled 'SEU Modeling Verification NMOS Depletion ModeLoad SRAMS." "

Joseph Wall (Biology) received a 1988 Ernest Orlando Lawrence Memorial Award from the Department of Energy in September, for his singular contributions to the development and application of the Scanning Transmission Electron Microscope, including the extensions of cellular microscopy to the resolution of single atoms, to the measurement of mass and shape of macromolecules, and the creation of the STEM user facility that makes this technology available to a broad community of scientists.
Emest Warburton (Physics) was awarded an Alexander von Humbolt research fellowship in August, to spend a year at the University of Heidelburg, West Germany, conducting electron scattering research.

Harvey Wegner (Physics) was elected a Fellow of the AAAS in February, for his role in the construction of BNL's Tandem Van de Graaff Accelerator for heavy ion physics and its recent interconnection to the Alternating Gradient Synchrotron.

Alfred Wolf (Chemistry) was elected to the National Academy of Sciences in May.

an open lecture series in the mornings, Brookhaven scientists covered current topics in such areas as physics, astronomy, chemistry, biology, medicine and applied science; in a limited number of afternoon internships, students worked in a research environment with sponsoring BNL scientists.

Ongoing student programs in 1988 included the 37-year-old Summer Student Program, in which 59 college juniors and seniors participated; the High School Honors Science Program sponsored by the U.S. Department of Energy, in which BNL hosted 52 of the nation's brightest high school students and six from other countries; Saturday tours of the Laboratory for seventh and eighth graders, sponsored by Associated Universities, Inc.; and the new Science and Engineering Research Semester for college students.

\section{Amrmative Action}

In the intern program sponsored by the Affirmative Action Office, four young careers were launched at Brookhaven, in the fields of facilities engineering, purchasing, biology/environmental protection and medical technology.

Continuing programs administered by Affirmative Action, designed to enhance minority interest in science and technical fields, included the introductory computer courses offered to adults and children in the Riverhead community; the SEEK mini-semester at Brookhaven for freshmen from the City College of New York; research apprenticeships for high school students; the Brookhaven Semester Program for students from historically black colleges; and the GEM program, for graduate students pursuing degrees in engineering. 


\section{Contracts \& Procurement}

The number of minority firms doing business at the Laboratory continued to increase, as did the dollars that Brookhaven invested in such companies.

\section{Patents Awarded}

In 1988 , ten patents were awarded to employees or former employees for devices invented or processes developed at Brookhaven.

\section{New Ambulance}

A brand-new ambulance was the 1988 addition to the five-vehicle fleet operated by the Fire \& Rescue Group of the Safety \& Environmental Protection Division. The ambulance, which carries a CPR Thumper device for performing cardiopulmonary resuscitation, is completely set up for basic life support fur transport of victims off site.

\section{4-Hour Banking}

In July, an agreement between Laboratory management and Barclays Bank of New York resulted in the opening of a 24-hour automated teller machine for cash and banking services, in the Berkner Hall lobby.

\section{Rebate from LILCO}

By reducing the Laboratory's electrical energy expenditures for lighting while maintaining safe and comfortable lighting levels, Brookhaven earned a $\$ 10,000$ rebate from the Long Island Lighting Company (LILCO). (Twenty percent of the Laboratory's electricity comes from LILCO. Brookhaven buys the other 80 percent from the New York Power Authority.)

The savings were realized through a lighting modifications project carried out by the Plant Engineering Division. Modifications included replacing 40-watt fluorescent lamps with 34-watt lamps that produce about the same light output, reducing energy consumption by 15 percent. Also, to provide the proper voltage and current to start and operate the fluorescent lamps, standard electromagnetic ballasts were replaced with electronic ballasts, which use about 35 percent less energy, run more quietly and last longer.

\section{Dial 282-Info}

A heavy snow fell overnight . . . impassable roads. Will the Laboratory open late? Since December 1987, Brookhaven employees have been able to get a quick answer to this kind of question by dialing the

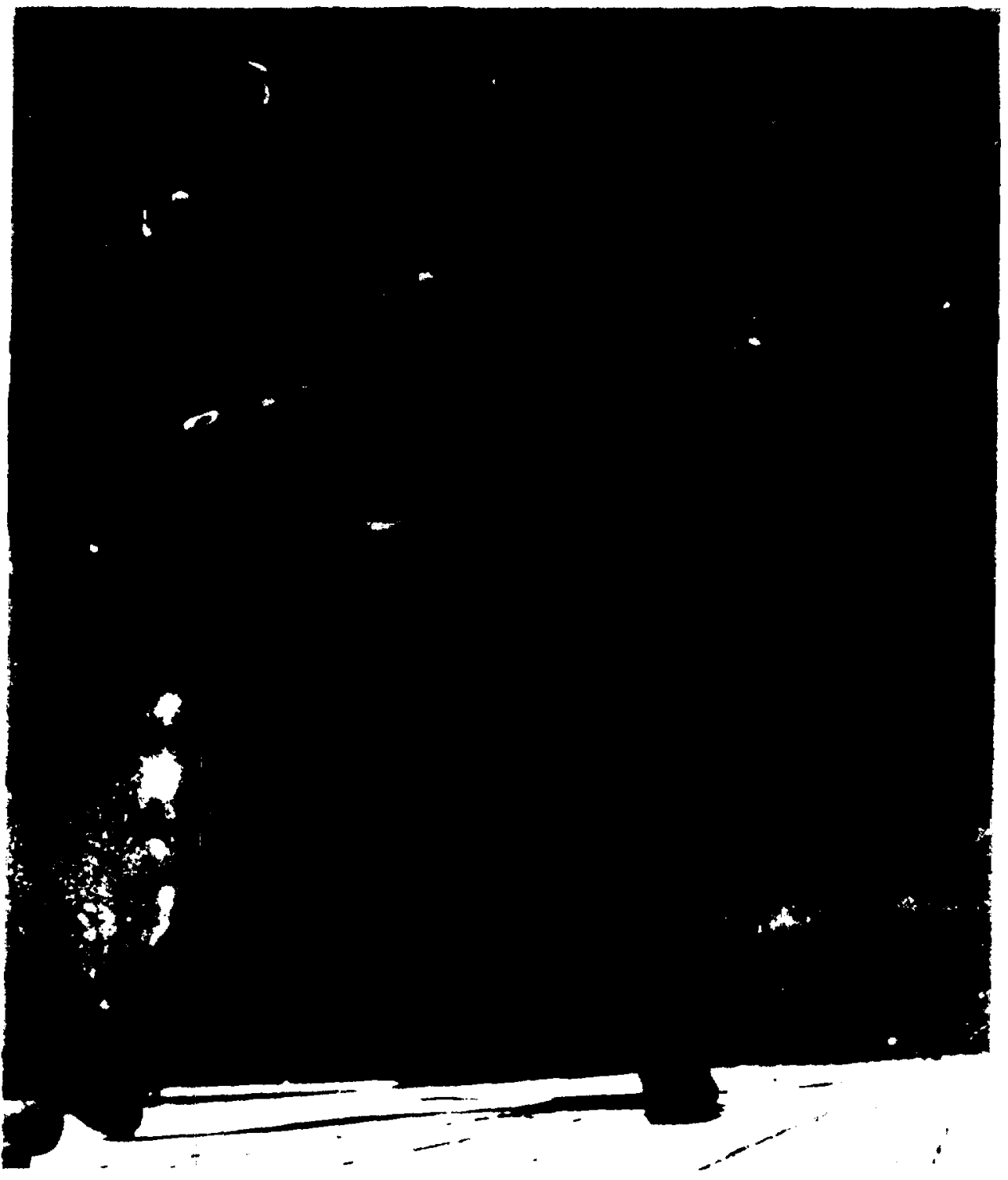

This is the view seen by each of the 28 participants as they practiced an Australian rappel down a 38-foot wall, during the Special Response Team Training held in June. Sixteen officers from Brookhaven's Police Group and 12 members of the Suffolk County Emergency Services sharpened their skills under the guidance of the Mobile Training Team from the Department of Energy's Central Training Academy in New Mexico. telephone number 282 -INFO and making use of a new information service.

\section{Training for Employees}

Throughout the year, the Laboratory conducts training programs intended to keep employees aware of the latest developments in their fields and to hone their skills to the highest levels (see photo). Among the areas in which employees were trained in 1988 were: 
- Safe removal of asbestos - To fulfill a new New York State law requiring the certification of all contractors, workers and supervisors involved with asbestos removal, 14 employees took this course, organized by the Plant Engineering Division, in January 1988.

- Digital electronics - Theory and practical application were the focus of this program arranged by the Personnel Division and presented to 45 employees from six departments in $\mathbf{1 6}$ half-day sessions from October 1987 to February 1988.

- Personal computers - Introductory courses on the IBM PC and on major software programs such as dBASE Ill Plus, Lotus 1-2-3, RBase System $V$ and WordPerfect are among those now offered by the Management Information Systems Division, which in May assumed responsiblity for this training, previously offered by the Personne] Division.

\section{Central Chilled Water Facility}

By year's end, work on the new Central Chilled Water Facility was well along. When completed in the spring of 1989, the facility will produce chilled water and compressed air to supply six buildings on the Laboratory site.

\section{BERA Activities}

In 1988, two new clubs were added to the list of over $\mathbf{4 0}$ organizations that fall under the aegis of the Brookhaven Employees Recreation Association (BERA). Boomerang enthusiasts formed the Boomerang Club, while automobile buffs created the Antique \& Classic Motoring Club. In addition, the Astronomical Society installed a new, membermade, 16-inch telescope in its onsite observatory.

\section{Team Safety}

In July 1988, the Laboratory kicked off its new Team Safety program, involving 25 teams of employees from three divisions:
Central Shops, Supply \& Materiel and Plant Engineering. In this ongoing incentive program, as each team reaches its time goal without any lost-time injuries, team members are rewarded for their safe practices with a luncheon. By the end of the year, after the progam had been in effect ten weeks, nine teams had reached the winners' lunch table.

\section{Child-Care Initiative}

BNL's Child-Care Initiative Committee, formed in January to evaluate Brookhaven employees' childcare needs, issued a report to the Laboratory Director in September. Based on the responses of almost 40 percent of BNL's employees to a Labwide survey, the committee recommended that Brookhaven establish an affordable child-care center on site, with adequate spaces for employees' children, and institute associated child-care benefits and policies. In response, the Director appointed a management committee to investigate the feasibility of establishing or sponsoring such a facility and asked the Personnel Division to consider the other recommendations.

\section{National Bridge Contest}

When put in Brookhaven's stress tester, a 31.17-gram balsa bridge built by a Colorado high school student supported 186 pounds taking top honors in the 11 th
National Model Bridge Contest organized at Brookhaven in May by the Tour Program in the Public Affairs Office. This was the second time the national contest was held at $\mathrm{BNL}$, in addition to the local contests hosted by the Tour Program each year.

\section{Administrative Actions}

John Axe was appointed to the newly created position of Scientific Program Head for the High Flux Beam Reactor, in April 1988.

W. Robert Casey was appointed Head of the Safety and Environmental Protection Division, as of January 1988.

Marilyn C. Galli was named Acting Division Manager of the Technical Information Division, in July 1988.

Geoffrey Hind became Chairman of the Biology Department in October 1987.

Walter Kato was named Chairman of the Department of Nuclear Energy in April 1988.

Norman Sutin was appointed Chairman of the Chemistry Department, in January 1988.

T. Laurence Truman became Associate Director for High Energy and Nuclear Physics, in September 1988.

Otto White was named Acting Assistant to the Director for Affirmative Action, in June 1988.

Employment Statistics
"Sclentific stoff
Scienttic Profoseional staft
- Nonscientific staff
Total

Percent of Tolal Employees

Females

16.6

23.1

\begin{tabular}{r}
1988 \\
645 \\
492 \\
2135 \\
\hline 3272 \\
16.6 \\
23.1 \\
gtaff.
\end{tabular}

\begin{tabular}{rr}
1987 & 1986 \\
670 & 603 \\
487 & 470 \\
2005 & 2064 \\
\hline 3261 & 3200
\end{tabular}

16.4 22.9

-Also inciudes research associates and visiting staff. 


\section{Finandil Deport}

The principal source of Brookhaven's funding is the U.S. Department of Energy (DOE). DOE accounted for $\$ 176.7$ million, or 79 percent, of BNL's $\$ 222.6$ million in operating funds in fiscal year (FY) 1988. Operating funds pay the costs of salaries and wages, fringe benefits, materials and supplies, and energy associated with the Laboratory's research programs.

Work for other federal agencies totaled $\$ 45.9$ million, the second largest category of Laboratory operating funds. This work was performed for the Nuclear Regulatory Commission, the Department of Defense, the Department of State, the Environmental Protection Agency, the National Institutes of
Health and the National Science Foundation.

DOE also provides capital equipment funding for instrumentation, scientific apparatus, computers and office equipment. In FY87, BNL's capital equipment budget totaled $\$ 18.4$ million, while in FY88 it rose to $\$ 20.5$ million.

A third aspect of Laboratory funding is for construction. In FY87, BNL's construction funds amounted to $\$ 25.2$ million, and in FY88, they dropped slightly to $\$ 24.5$ million.

Primarily, the decrease reflects the completion of the Phase II expansion of the National Synchrotron Light Source and the winding down of the Central Chilled Water Facility Project. Construction pro-

\section{BNL Cost Elements}

\section{Fringe Baneftits}

Material \& supplice

Electric Power

Heating Fual

Salary \& Wages

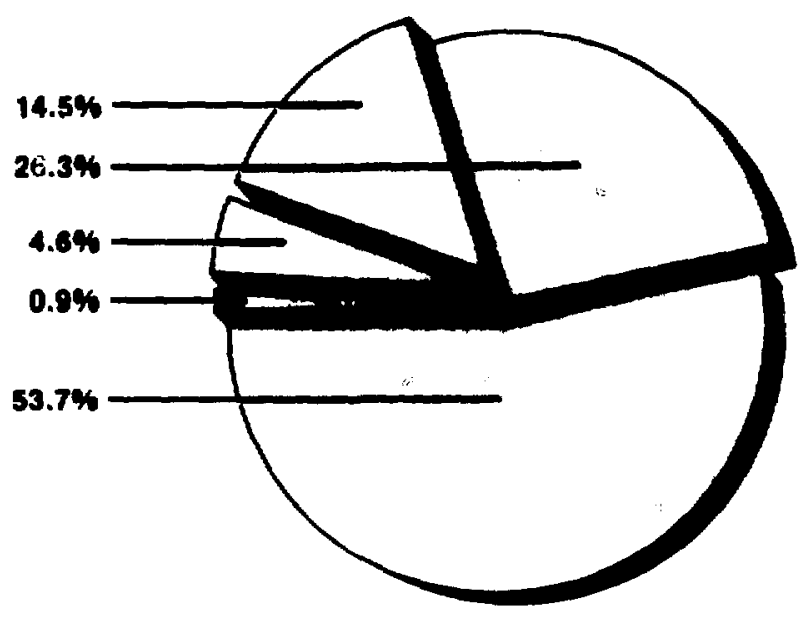

jects initiated in FY86 and ongoing into FY88 include the AccumulatorBooster at the Alternating Gradient Synchrotron and fire protection improvements. Environmental improvements initially funded in FY88 are also under way.

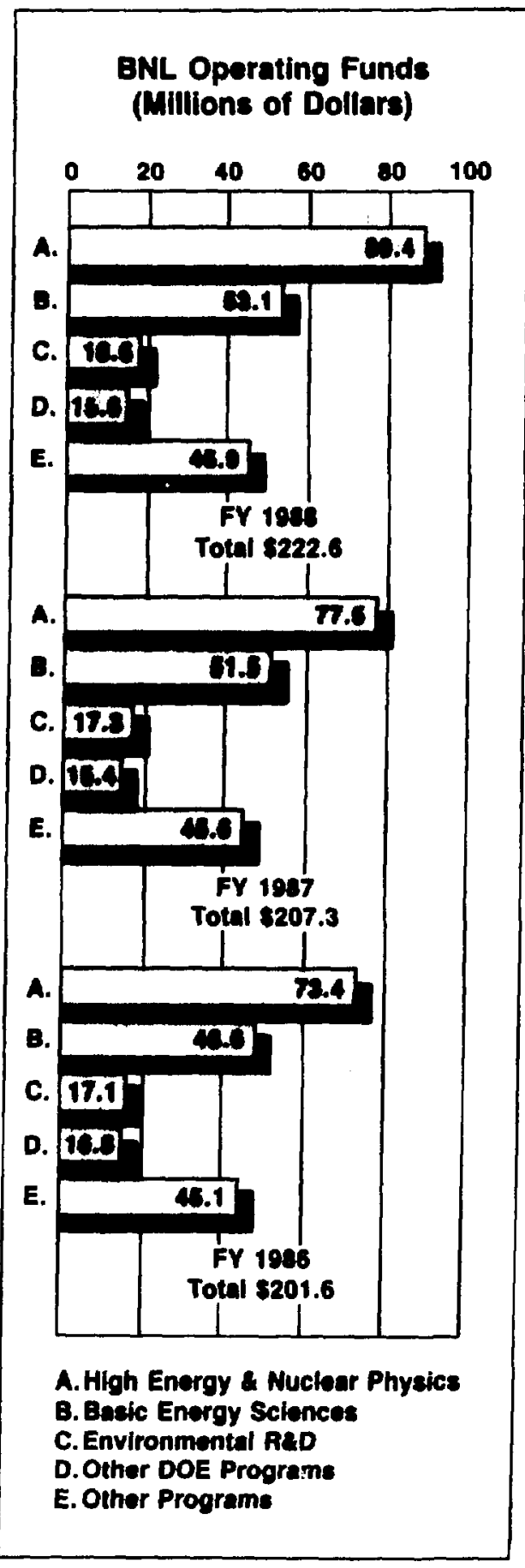




\section{Organization}

\section{Directorate}

Nicholas P. Samjos

Director

Martin Blume

Deputy Director

*Associate Director for Basic Energy Sciences

Seymour Baron

Associate Director for Applied Programs

Henry C. Grahn

Associate Director for Administration

E. Parke Rohrer Associate Director for Management and Physical Plant

Richard B. Setlow

Associate Director for Life Sciences

T. Laurence Trueman

Associate Director for High Energy and Nuclear Physics

Gerald C. Kinne

Assistant Director for Reactor, Safety $\&$ Security

\section{Department Chairmen}

Peter D. Bond Physics

Arjun D. Chanana Medical

Eric B. Forsyth Accelerator Development

Geofirey Hind Biology

Walter Y. Kato Nuclear Energy

Michael L. Knotek National Synchrotron Light Source

Derek I. Lowenstein Alternating Gradient Synchrotion

Bernard Manowitz Applied Scitree

Ronald F. Peierls Applied Mathematics

Norman Sutin Chemistry Department

\section{Division Heads}

Michael H. Brooks

Reactor

W. Robert Casey

Safety \& Environmental Protection

Veljko Radeka

Instrumentation

\section{Managers and Office Heads}

Robert Bacharach

Management Information Systems

Anne S. Baittinger

Public Affairs

Robert D'Angio

Personnel \& Labor Relations

* Marilyn C. Galli

Technical Information

Michael M. Goldman

Legal Counsel

Michael A. Guacci

Supply \& Materiel

Mark O. Israel

Fiscal

John B. King

Contracts \& Procurement

John B. Laurie

Photography \& Graphic Arts

Alred Mahlmann

Plant Engineering

William Marcuse

Research \& Technology

Applications

Bernard J. McAlary

Business Manager

Robert B. Palmer and

Claudio Pellegrini

Center for Accelerator Physics

Donald J. Robbins

Budget

E. Parke Rohrer

Project Management

Larry J. Runge

Safeguards \& Security

Marvin Shear

Quality Assurance

Richard J. Spellman Central Shops

William S. Webster

Staff Services

*Otto White Jr.

Affirmative Action

"Acting

Organization effective as of

September 30, 1988 


\section{BNL'ers}

Front Cover (from left): Sam Morris. Arlean Vanslyke, Martin Leach, Robert Brown, Pat Glynn, Harvey Richardson Jr., C.ynthia Morgan,

Edwin Taylor, Irene Rosati. Helen Kondratuk, Pamela Gibbs.

Inside Front Cover: Harriet Martin, Morton Rosen, Rebecca Williams, Garman Harbottle. Eric Forsyth, Thomas Ludlam, Don David, James Briggs. Herbert Hildebrand.

Inside Back Cover: Elinor Norton, Frances Scesny, Alan Kuehner, Frank Marotta, Richard Setlow, Frank Kito.

Back Cover: Rudolph Damm, M. Kay Hunt, Martin Fallier, Kenneth Testa, Edward Murphy, Gus Prince, Michele Dickerson, Janet Sillas.

Page 15: Paula Hughes, Gordon Danby, Richard Skelton, Eugene Kaplan, Hue-Anh Pham, Ahovi Kponou, Vinnie LoDestro.

Page 23: Harvey Lotko, Vernon Hughes, Juanita Heyliger, Ernest Courant, Albert Prodell, Susan Norton, Stephanie Lamontagne, Michael Nekulak.

Page 29: Irving Feigenbaum, Maurice Goldhaber, Pavel Rehak, Fern Simes, Howard Gordon, Marilyn McKeown, Pierrot Bichoneau, Martin Van Lith, John Axe Jr.

Page 35: Catherine Green, Victoria Mclane, Leslie Fishbone, William Higinbotham, Jerry Cadwell, Bruce Penn, Bonnie Bittner.

Page 43: Richard Wilke, John McNeil, Arnold Moodenbaugh, Robert Jones, Allen Goland, Lawrence Kukacka, Meyer Steinberg.

Page 51: James Murphy, Judith Thompson, Gaetano Vignola, Thomas Dickinson, Gwyn Williams, Claudio Pellegrini, William Thomlinson, Jeannette Thiede.

Page 57: Lee Walcott, David Comstock, Blair Munhofen, Bonnie Wesolowski, Sophie Kostuk, Conrad Koehler Jr., Evelyn Ritter, Richard Becker, Bruce Brunschwig.

Page 65: Herbert Susskind, William Harold, Prantika Som, Eugene Cronkite, (seated) Ted Johnson, Jean Matkovich, Lydia Schlichtcroll, (standing) Veronica Brooks, Noelwah Netusil, Bernice Armstrong, Robert Brown, Joseph Cardamone.

Page 71: Dieter Schneider, Thomas Langdon, Edward Caruso, Frances Burr, Virginia Pond, Harold Siegelman, Neal Tempel, Myron Ledbetter.

Page 77: George Sintchak, John Nicolellis, George Walczyk, Frederick Strier, Ronald Wittlock, Kurt Fuchel, David Rorer.

Page 87: Michele Haller, Andrew Feldman, Spider Ligon, Edward Sujeski Jr., Edward Miezianka, Elinor Adams, April Donegain, Yreana-Renée Flack, Frank De Rosa. 


\section{Credits}

Editor: Mona S. Rowe

Writer-Editors: Anita Cohen, Liz Seubert

Technical Editor: J.B. Horner Kuper

Cover Design: Alan Schmidtchen

Graphic Design and Composition: Photography and Graphic Arts Division, with assist ance from BURMAR Technical Corporation of Albertson. New York.

Photography: All photography by Roger Stoutenburgh, except for photos on pp. 2, 3, 5, 7, 8, 9 (Setlow), 10 (Blume), 11 (Palmer and Robbins), 12, 14, 42 and 72 by Morton Rosen and on p. 25 by Peter Horton.

\section{Produced by the Public Affairs Office}

Anne S. Baittinger, Manager

For more information about work described in this report or for information about Brookhaven, contact the Public Affairs Office, Brookhaven National Laboratory, Upton, Long Island, New York 11973.

Brookhaven National Laboratory is operated by Associated Universities, Inc., under contract No. DE-AC02-76CH00016 with the United States Department of Energy. Brookhaven is an affirmative action/equal opportunity employer.

Report No. BNL 52165. DOE/OSTI-4500-INTERIM 3, distribution categories UC400 and UC-500 - general, miscellaneous and progress reports (nuclear and nonnuclear). Printed in the United States of America. Available from the National Technical Information Service, U.S. Department of Commerce, 5285 Port Royal Road, Springfield, VA 22161 . NTIS price codes: printed copy - A05, microfiche copy - A01.

This report was prepared as an account of work sponsored by an agency of the United States Government. Neither the United States Government nor any agency thereof, nor any of their employees, nor any of their contractors, subcontractors, or their employees makes any warranty, express or implied, or assumes any legal liability or responsibility for the accuracy, completeness, or usefulness of any information, apparatus, product, or process disclosed, or represents that its use would not infringe privately owned rights. Reference herein to any specific commercial product, process, or service by trade name, trademark, manufacturer, or otherwise, does not necessarily constitute or imply its endorsement, recommendation, or favoring by the United States Government or any agency, contractor or subcontractor thereof. The views and opinions of authors expressed herein do not necessarily state or reflect those of the United States Government or any agency, contractor or subcontractor thereof. 